Effects of prenatal exposure to cancer

KU LEUVEN treatment on neurocognitive development An MRI and ERP study

\title{
Jeroen Blommaert
}

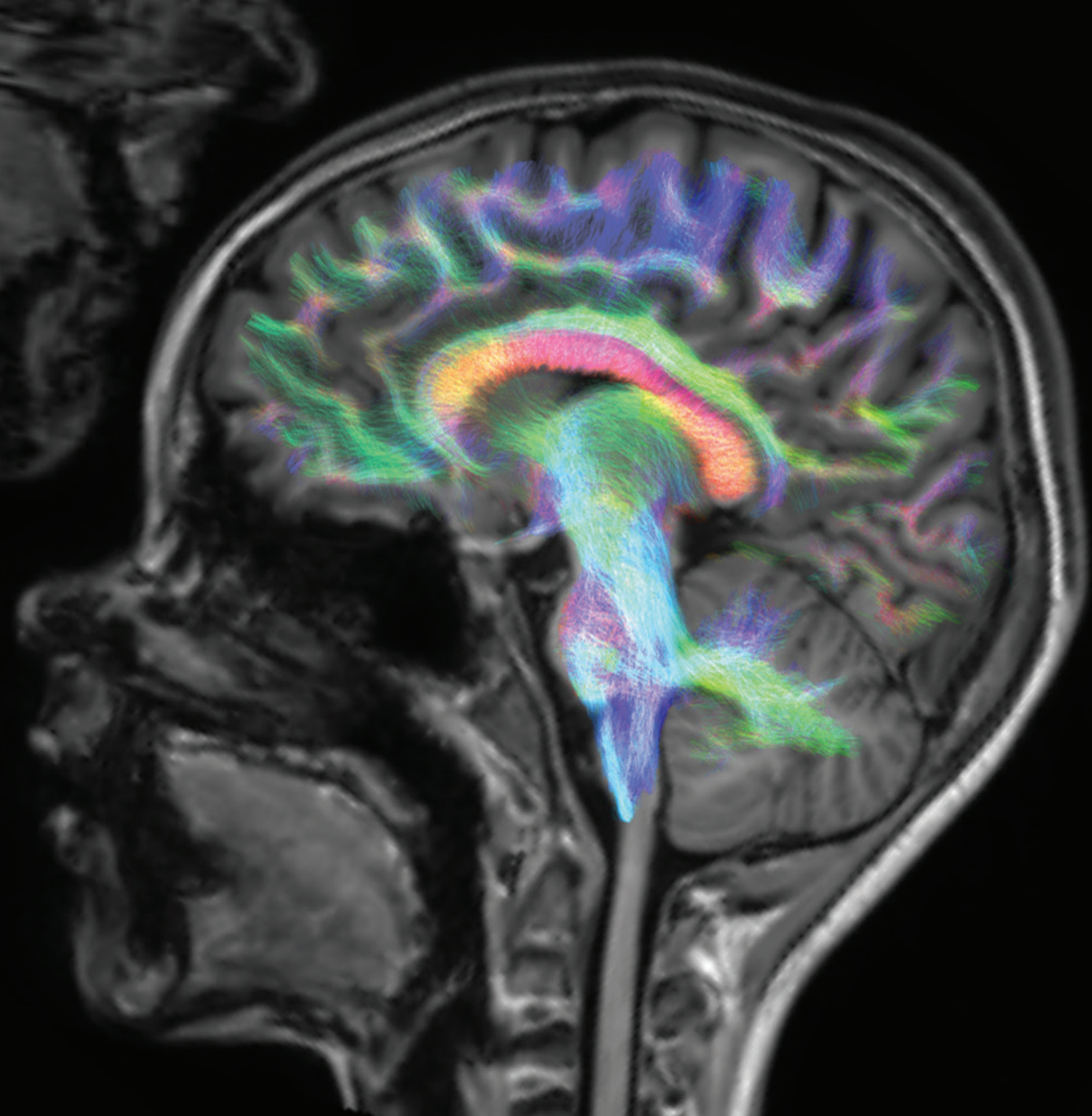


KU Leuven

Group Biomedical Sciences

Faculty of Medicine

Department of Oncology

\section{Effects of prenatal exposure to cancer treatment on neurocognitive development} An MRI and ERP study

Jeroen BLOMMAERT

Jury:

Supervisor:

Prof. Dr. Frédéric amant

Co-supervisors:

Dr. Sabine Deprez

Prof. Dr. Kristel Van Calsteren

Chair examining committee:

Prof. Dr. Marc Fransen

Chair public defense:

Prof. Dr. Baki Topal

Jury members:

Prof. Dr. Patrick Dupont

Prof. Dr. Katrien Jansen

Dr. Monica Fumagalli

Dr. Maarten Mennes

Dissertation presented in partial fulfilment of the requirements for the degree of Doctor in Biomedical Sciences 
This project has received funding from the European Union's Horizon 2020 research and innovation program (European Research council, grant №647047), Foundation against cancer (Stichting tegen kanker, grant №2014-152) and Research Foundation Flanders (FWO, grant №11B9919N). The computational resources and services used in this work were provided by the VSC (Flemish Supercomputer Center), funded by the FWO and the Flemish Government - department EWI

(C) 2020 by Jeroen Blommaert

Alle rechten voorbehouden. Niets uit deze opgave mag vermenigvuldigd en/of openbaar gemaakt worden door middel van druk, fotokopie, microfilm, electronisch of op welke andere wijze ook zonder voorafgaande schriftelijke toestemming van de uitgever.

All rights reserved. No part of this publication may be reproduced in any form by print, photoprint, microfilm, or any other means without written permission from the publisher. 


\section{CONTENTS}

List of Figures $\quad$ iv

List of Tables

Abstract $\quad$ vii

Beknopte sammenvatting $\quad$ ix

1 Introduction 1

1.1 Risks of cancer in pregnancy for the offspring . . . . . . . . . . . . . . . . . . 1

1.2 Cancer, chemotherapy and the brain . . . . . . . . . . . . . . . 2

1.3 The developing brain . . . . . . . . . . . . . . . . . . . . . . 5

1.4 Multimodal neuroimaging . . . . . . . . . . . . . . 6

1.5 Conclusions . . . . . . . . . . . . . . . . . . . . . . . . . . 7

2 Objectives $\quad 8$

3 Methodology 10

3.1 Participants . . . . . . . . . . . . . . . . . . . . . . . . 10

3.2 Event-related potentials . . . . . . . . . . . . . . . . . . . . . . 12

3.3 Magnetic resonance imaging . . . . . . . . . . . . . . . . . . . 17

3.4 Graph theory . . . . . . . . . . . . . . . . . . . . . . . . . . 21

4 Age-dependent brain volume and neuropsychological changes after chemotherapy in breast cancer patients.

4.1 Abstract . . . . . . . . . . . . . . . . . . . . . 25 
4.2 Introduction . . . . . . . . . . . . . . . . . . . . 26

4.3 Methods . . . . . . . . . . . . . . . . . . . . . . . . . . 27

4.4 Results . . . . . . . . . . . . . . . . . . . . . . . . . . . . . . . . . 29

4.5 Discussion . . . . . . . . . . . . . . . . . . . . . . . . . . . 40

5 Long-term impact of prenatal exposure to chemotherapy on executive functioning: an ERP study 43

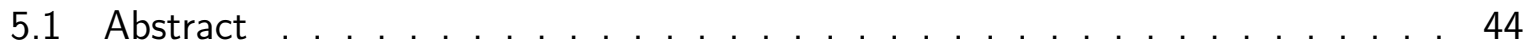

5.2 Introduction . . . . . . . . . . . . . . . . . . . 45

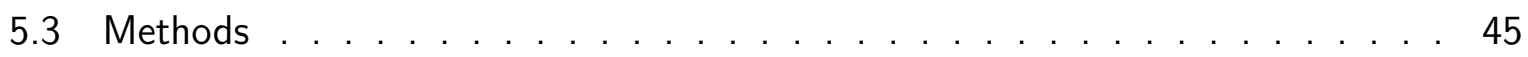

5.4 Results . . . . . . . . . . . . . . . . . . . . . . . . . . . . . . 48

5.5 Discussion . . . . . . . . . . . . . . . . . . . . . . . 57

5.6 Conclusions . . . . . . . . . . . . . . . . . . . . . . . . . . . . . . . . . 59

6 The impact of cancer and chemotherapy during pregnancy on child neurodevelopment: a multimodal neuroimaging analysis $\quad \mathbf{6 0}$

6.1 Abstract . . . . . . . . . . . . . . . . . . . . . . . . 61 61

6.2 Introduction . . . . . . . . . . . . . . . . . 62

6.3 Methods . . . . . . . . . . . . . . . . . . . . . 63

6.4 Results . . . . . . . . . . . . . . . . . . . . . . . 66

6.5 Discussion . . . . . . . . . . . . . . . . . . . . . . . 76

6.6 Conclusions . . . . . . . . . . . . . . . . . . . . . . . . . . . 78

7 Discussion $\quad 79$

7.1 Cancer, chemotherapy and the brain . . . . . . . . . . . . . . . 80

7.2 Neurodevelopment after prenatal exposure to cancer treatment . . . . . . . 81

7.3 Limitations . . . . . . . . . . . . . . . . . . . . . . . . . . . . . . . 83

7.4 Future perspectives . . . . . . . . . . . . . . . . . . . 85

7.5 General conclusion . . . . . . . . . . . . . . . . . . 87

8 Appendix $\quad 89$

8.1 Supplementary tables to chapter $4 \ldots \ldots$. . . . . . . . . . . . . . 90

8.2 Supplementary materials to chapter $6 \ldots \ldots$. . . . . . . . . . . . . . . . . . . . . . 94

Acknowledgements, personal contribution and conflict of interest statements 100

$\begin{array}{ll}\text { Bibliography } & 102\end{array}$

$\begin{array}{ll}\text { Curriculum vitae } & 126\end{array}$ 
Dankwoord 


\section{LIST OF FIGURES}

1.1 Potential mechanisms of altered neurodevelopment after prenatal exposure to maternal cancer (therapy) . . . . . . . . . . . . . . . . . . . . . . 4

1.2 Stages of prenatal brain development. . . . . . . . . . . . . . . 6

3.1 Overview of neuromaging modalities used throughout this dissertation. . . . . 11

3.2 ERP paradigms. . . . . . . . . . . . . . . . . . . . . . . . . . . . 13

$3.3 \mathrm{Go} /$ Nogo grand average potentials. . . . . . . . . . . . . . . . . . . . 14

3.4 Posner grand average potentials. . . . . . . . . . . . . . . . . . . 16

3.5 Principles of diffusion MRI . . . . . . . . . . . . . . . . . . . . . . . . . . . . . . . . . . 19

3.6 The diffusion signal in different brain tissues. . . . . . . . . . . . . . . . . 20

3.7 Visualization of a brain connectome. . . . . . . . . . . . . . . . . . . 22

4.1 Within-group volumetric changes of all groups, independent of age, comparing time point $t 2$ with baseline. . . . . . . . . . . . . . . . . 35

4.2 Comparison of the volumetric changes in young and old women after cancer therapy. 37

4.3 Correlation of changes in cognitive scores with changes in brain volume of young and old $\mathrm{C}+$ patients. . . . . . . . . . . . . . . . . . . . . . . . . . . . . . . . . . . . 39

5.1 Event-related potentials of the Go/Nogo paradigm. . . . . . . . . . . . . . . 53

5.2 Parietal event-related potentials of the Posner paradigm . . . . . . . . . . . 55

5.3 Occipital event-related potentials of the Posner paradigm. . . . . . . . . . . . 56

6.1 Flow diagram of recruitment for this study. . . . . . . . . . . . . . . . 67

6.2 Observed differences in WM microstructure. . . . . . . . . . . . . . . . . 73

6.3 Relationship of brain differences with maternal clinical history. . . . . . . . . . 75 
8.1 Observed differences in WM microstructure at trend level. 


\section{LIST OF TABLES}

4.1 Overview of the total population size and subdivision into samples . . . . . . 30

4.2 Demographics and clinical characteristics of the study population. . . . . . . . 31

4.3 Summary of the neuropsychological assessments. . . . . . . . . . . . . . . . 32

4.4 Neuropsychological assessment of the young and old C+ patients. . . . . . . . 34

5.1 Population characteristics. . . . . . . . . . . . . . . . . . . 50

5.2 Maternal tumor types and treatment during pregnancy. . . . . . . . . . . 51

5.3 Behavioral performance on both paradigms. . . . . . . . . . . . . . . . . . 52

5.4 List of all significant time intervals in the event-related potentials of both paradigms. . . . . . . . . . . . . . . . . . . . . . . . . 54

6.1 Population characteristics. . . . . . . . . . . . . . . . . . 68

6.2 Maternal disease and treatment during pregnancy. . . . . . . . . . . . . 70

6.3 Neuropsychological outcomes of the child . . . . . . . . . . . . . . . . 71

6.4 Cortical morphometric measures. . . . . . . . . . . . . . . . . . . . . . . . . 71

8.1 Neuropsychological assessment of the C- patients and healthy controls. . . . . 91

8.2 MNI coordinates, cluster extent and statistical values of significant clusters from the DBM analysis described in Figure 4.1. . . . . . . . . . . . . . . . . . 92

8.3 MNI coordinates, cluster extent and statistical values of significant clusters from the DBM analysis described in Figure 4.2 . . . . . . . . . . . . . . . . . 93

8.4 Image quality metrics . . . . . . . . . . . . . . . . . . . . . . . . . . . . . 94

8.5 Global brain volumes. . . . . . . . . . . . . . . . . . . . . . . . . . . . . . 94 


\section{ABSTRACT}

About 1 in 1000 pregnancies is complicated by maternal cancer, a diagnosis which confronts families and caregivers with difficult medical and ethical decisions, with potential long-lasting consequences for both mother and child. While studies have indicated reassuring outcomes for the child after different therapies during pregnancy, subtle differences in birthweight, cardiac functioning and neuropsychological outcome have raised questions on the long-term effects of prenatal exposure to maternal cancer and its treatment.

The main aim of this thesis is to provide solid data on the long-term effects of prenatal exposure to cancer and its treatment on neurocognitive development, with particular focus on the use of chemotherapy during pregnancy. Furthermore, we want to explore which treatment-related and secondary factors might play a role in the observed neurodevelopmental effects. For instance, many children are born preterm after cancer-complicated pregnancies, which has been associated with lower IQ scores and an increased risk of behavioral and neurocognitive problems.

Advanced multimodal neuroimaging techniques, measuring different functional and structural characteristics of the brain, are used to investigate the multifaceted nature of neurodevelopment in these children. This information is then combined with data on mother and child from the International Network on Cancer, Infertility and Pregnancy (INCIP) registration and follow-up studies, in order to relate neuroimaging findings to the oncological and obstetrical history, as well as to the neuropsychological outcome.

First, we investigated the impact of chemotherapy on brain volume in a longitudinal cohort of 121 young and older, non-pregnant, women with breast cancer, of whom 72 treated with chemotherapy, alongside 59 controls. We observed age-dependent cognitive decline and white 
matter volume expansion, potentially caused by chemotherapy-induced neuroinflammatory processes, with younger patients showing less cognitive decline and more white matter volume increase. These results suggest the younger brain to have a neuroprotective response to chemotherapy-induced neurotoxicity.

Next, we applied event-related potentials to evaluate the development of executive functioning in 20 nine-year-old children who were prenatally exposed to chemotherapy, comparing with controls who where matched on a 1:1 ratio based on age, sex and gestational age at birth. We found children with prenatal chemotherapy exposure to have an impacted development of response inhibition and spatial attention. Moreover, both prenatal chemotherapy exposure and prematurity were found to affect neurocognitive processes of conflict monitoring. These findings indicate that cancer and/or chemotherapy during pregnancy might indeed impact neurodevelopment of the child, not limited to the effect of prematurity.

Finally, multimodal MRI techniques were used to assess structural and functional brain development of 42 children born to cancer-complicated pregnancies, compared to matched controls. Cancer during pregnancy was associated with local differences in grey and white matter structure, but was not found to significantly affect functional connectivity or brain organization. While platinum derivatives showed an increased impact on gyrification of the left superior temporal gyrus, chemotherapy during pregnancy in general was not associated with a worse outcome.

In summary, the work of this thesis describes a subtle impact of maternal cancer and its treatment on neurodevelopment of the child at the age of nine years. Although we, as well as others, observed the neurotoxic impact of chemotherapy in cancer patients, chemotherapy during pregnancy showed only a limited effect on the neurodevelopment of the child. Secondary mechanisms, such as the psychosocial impact of a cancer diagnosis during pregnancy, might have large contributions to the observed neurodevelopmental differences, and have to be explored in future research. Balancing the benefits of treating cancer during pregnancy against the subtle neurocognitive impact observed in this thesis, the current data favour cancer treatment during pregnancy, including chemotherapy, when clinically indicated. 


\section{BEKNOPTE SAMENVATTING}

Ongeveer 1 op 1000 zwangere vrouwen wordt geconfronteerd met een kankerdiagnose. Deze diagnose stelt zowel patienten als hun zorgverleners voor een reeks moeilijke beslissingen, die mogelijks zware gevolgen kunnen hebben voor zowel de moeder als het ongeboren kind. Hoewel de bestaande literatuur overwegend geruststellende uitkomsten beschrijft voor het kind nadat de moeder kankerbehandeling tijdens de zwangerschap onderging, zijn er wel enkele subtiele verschillen gevonden in geboortegewicht, hartfunctioneren en prestaties op neuropsychologische testen. Bijgevolg is het belangrijk om na te gaan of kankerbehandeling tijdens de zwangerschap op langere termijn de gezondheid van het kind schaadt.

Het doel van dit doctoraatsproefschift is om duidelijkheid te scheppen over de langetermijn gevolgen van kankerbehandeling tijdens de zwangerschap op de hersenontwikkeling van het kind, in het bijzonder wanneer chemotherapie gebruikt werd tijdens de zwangerschap. Daarnaast willen we ook in kaart brengen welke andere factoren, rechtstreeks en onrechtstreeks verbonden aan kanker tijdens de zwangerschap, een rol spelen in de hersenontwikkeling van deze kinderen. Prematuriteit, bijvoorbeeld, komt vaak voor bij deze kinderen en is in het verleden al gekoppeld aan het vaker voorkomen van gedrags- en neurocognitieve problemen.

Geavanceerde beeldvorming en -verwerkingstechnieken worden doorheen dit proefschrift gebruikt om hersenontwikkeling te onderzoeken. Deze technieken kunnen verschillende aspecten van hersenstructuur en functioneren in kaart brengen, om zo herenontwikkeling in al zijn facetten te kunnen bestuderen. Deze bevindingen rond hersenontwikkeling worden dan teruggekoppeld naar de behandeling van de moeder en de neuropsychologische uitkomsten van het kind.

Allereerst hebben we onderzocht hoe chemotherapie hersenvolume kan veranderen, door dit te 
bestuderen in een groep van 121 jonge en oudere, niet zwangere, vrouwen met borstkanker. $\mathrm{Na}$ kankertherapie zagen we wijdverspreide uitzetting van de witte stof en een slechter cognitief functioneren. Dit wijst er mogelijks op dat chemotherapie een ontsekingsreactie in de hersenen kan veroorzaken. Jongere vrouwen toonden meer witte stof uitzetting en minder cognitieve schade, wat zou kunnen er op wijzen dat het jonge brein een beschermende reactie heeft tegen de schadelijke effecten van chemotherapie

Vervolgens gebruikten we event-related potentials (ERP) om de ontwikkeling van executieve functies te bestuderen in 20 9-jarige kinderen waarvan de moeder tijdens de zwangerschap chemotherapie toegediend kreeg, en vergeleken dit met 20 kinderen van gezonde moeders. Deze techniek meet elektrische activiteit op de hoofdhuid tijdens het uitvoeren van een taak, om zo de elektrische activiteit van de hersenen tijdens deze taak te bekijken. We vonden dat kinderen geboren na kanker tijdens de zwangerschap het slechter deden op vlak van reactieremming en verschuiving van aandacht. Daarnaast vonden we dat zowel kanker tijdens de zwangerschap als prematuriteit een effect had op conflictbewaking. Deze bevindingen duiden er op dat kanker en/of chemotherapie tijdens de zwangerschap inderdaad een impact kunnen hebben op de hersenontwikkeling van het kind, en dit niet alleen doordat deze kinderen vaker prematuur geboren worden.

Tenslotte combineerden we verschillende MRI technieken om hersenstructuur en -functioneren te bestuderen in 42 9-jarige kinderen geboren na kanker tijdens de zwangerschap, in vergelijking met 42 kinderen van gezonde moeders. Kanker tijdens de zwangerschap had een invloed op lokale witte en grijze stofstructuur, maar had geen impact op de organizatie van de hersenen of functionele connectiviteit. Hoewel therapie met platinumderivaten een verhoogde impact vertoonde op de hersenplooiing van de linkse superieur temporele gyrus, vormde chemotherapie in het algemeen tijdens de zwangerschap geen verhoogd risico voor de hersenontwikkeling.

Tot slot, de verschillende bevindingen in dit proefschrift beschrijven een subtiele impact van kanker tijdens de zwangerschap op de hersenontwikkeling van het kind. Hoewel we, net zoals andere studies, hersenschade na chemotherapie vonden in patienten met kanker, had chemotherapie tijdens de zwangerschap slechts minimale impact op de hersenontwikkeling van het kind. Andere factoren, zoals de psychosociale impact van een kankerdiagnose tijdens de zwangerschap, spelen wellicht een grote rol in de verschillen die we waarnamen in de hersenontwikkeling van deze kinderen, en moeten in detail bestudeerd worden in toekomstige studies. Alle bevindingen van deze thesis samengenomen, toont dit werk dat een effectieve behandeling van kanker, inclusief gebruik makende van chemotherapie, tijdens de zwangerschap mogelijk is, zonder majeure effecten op de hersenontwikkeling van het kind. 


\section{CHAPTER}

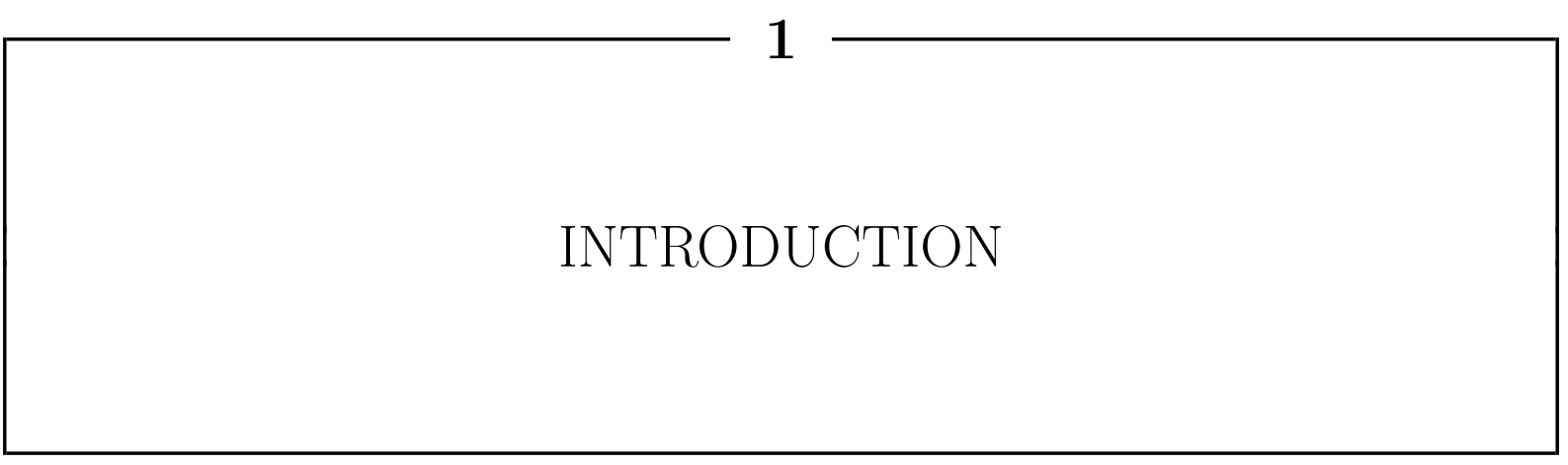

The diagnosis of cancer during a pregnancy immediately confronts health carers and patients with extremely difficult medical and ethical decisions ${ }^{1}$. The pros and cons of treating the patient, premature induction or treatment delay need to be considered, taking into account both maternal and fetal health. It is estimated that 1 in every 1000 pregnancies is complicated by a cancer diagnosis ${ }^{2}$. This incidence is expected to rise with current trends of older age at motherhood and increased use of non-invasive prenatal testing $3 ; 4$.

Throughout this chapter, we will introduce the key concepts of this dissertation. First we will provide a short overview of current literature on the offspring's outcomes after maternal cancer during pregnancy, as well as current clinical practice. Then, we will discuss potential mechanisms through which maternal cancer and chemotherapy could potentially affect neurodevelopment. Next, we will discuss how multimodal neuroimaging could provide tools for investigating neurodevelopment in children born after cancer-complicated pregnancy. Finally, we will summarize these core concepts, which will allow us to define our objectives in chapter 2 .

\subsection{Risks of cancer in pregnancy for the offspring}

Pre-clinical and clinical studies have indicated that an effective cancer treatment during pregnancy is often feasible without major short-term effects on the offspring ${ }^{5-11}$. Surgery is often 
possible outside the pelvic area with proper foetal monitoring, radiotherapy can be performed when the cumulative fetal radiation dose can limited to maximum 50-100mGy and different chemotherapeutic agents can be administered after the first trimester ${ }^{12}$. Chemotherapy is avoided during the first trimester, as its interaction with ongoing organogenesis is linked to an increased risk for fetal malformations ${ }^{12 ; 13}$. These insights and guidelines have lead to an increasing number of patients being treated during pregnancy, with nowadays $71 \%$ of women receiving treatment during pregnancy of which $62 \%$ receive chemotherapy ${ }^{2}$.

While previous studies overall showed promising outcomes, regarding the offspring, after cancer treatment during pregnancy, some subtle effects were observed. These studies describe a higher occurrence of disharmonic IQ profiles ${ }^{5}$, a lower verbal IQ ${ }^{14}$, subclinical cardiac effects (lower ejection and shortening fraction and lower velocities in the basal segment of the intraventricular septum and mitral valve) $)^{5 ; 6}$ and a higher rate of children being born small for gestational age $(\mathrm{SGA})^{2}$. Moreover, cancer during pregnancy is associated with a high rate of (late-)preterm birth, which has been shown to impact cognitive and behavioural functioning ${ }^{5 ; 6}$.

One mouse study showed potential neurotoxic effects of prenatal exposure to high dose vinblastine and doxorubicin treatment ${ }^{15}$. This study observed lesions in the neocortical subventricular zone as well as irregular thickening of the cortex in prenatally exposed mice, with some mice also showing cortical lamination defects. Such early insult to cortical organization might increase the risk for developing conditions such as polymicrogyria or focal cortical dysplasia ${ }^{16}$. So far, only one study has investigated the effect of prenatal chemotherapy exposure on brain development in humans, finding no relation of therapy with neonatal brain volumes ${ }^{17}$.

Currently, there is a lack of long-term data on the offspring's outcomes after maternal cancer during pregnancies. Previous studies have either included children at heterogeneous ages $5 ; 10$ or within the first life years $6 ; 17$. The longest follow-up study till date has assessed the effect of maternal cancer on cognition, behaviour and cardiac functioning in 132 children and matching controls at the age of 6 years ${ }^{14}$.

\subsection{Cancer, chemotherapy and the brain}

Chemotherapy has been linked to neurocognitive impairments in different childhood ${ }^{18 ; 19}$ and adult ${ }^{20-22}$ cancer populations. In particular the cognitive impact of chemotherapy on higher cognitive functions such as executive functions have often been reported 18;21;23;24. Moreover, chemotherapy has been linked to leukoencephalopathy ${ }^{25 ; 26}$ and changes in white matter (WM)

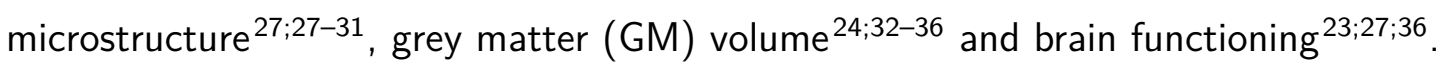


Unfortunately, the mechanisms through which these neurocognitive changes occur, remain largely unclear $21 ; 37 ; 38$. First some chemotherapeutic agents, such as fluorouracil, Methotrexate and cisplatin, have been found to cross the blood-brain barrier, potientially causing direct neurotoxicity ${ }^{39-41}$. Second, chemotherapeutic agents and immunotherapy might induce neural damage through indirect neuro-inflammatory processes due to an upregulated peripheral immune response ${ }^{42}$. Third, oxidative stress, as well as other processes of DNA damage, might induce neurodegeneration. Mechanisms of oxidative stress have been associated with different chemotherapeutics, such as methothrexate, cis- and carboplatin, doxorubicin and cyclophosphamide ${ }^{18 ; 43}$. Finally, chemotherapeutic agents are designed to reduce cell division within the tumour. However, when these chemotherapeutic agents pass through the blood-brain barrier they might also inhibit neurogenesis. This might be especially harmful in the context of cancer in pregnancy, as rapid cell division is one of the key aspects in prenatal development ${ }^{44}$. Recently, Ahles and colleagues ${ }^{20}$ provided a model for explaining this so-called "chemobrain". In this model, the treatment and tumor interact with psychosocial, lifestyle, genetic, physological and metabolic factors, emphasizing the complex nature of these neurocognitive effects.

While studies on the effect of cancer therapy on the brain provide useful insights for the potential effects of in utero exposure to cancer therapy on the brain, they cannot be directly translated to this population. Indeed, additional mechanisms such as ongoing brain development, neuroplasticity, the placental barrier, fetal urine reabsorption as well a the immature fetal metabolism and blood-brain brain barrier all play a role (Figure 1.1$)^{8 ; 9 ; 11 ; 44}$. 


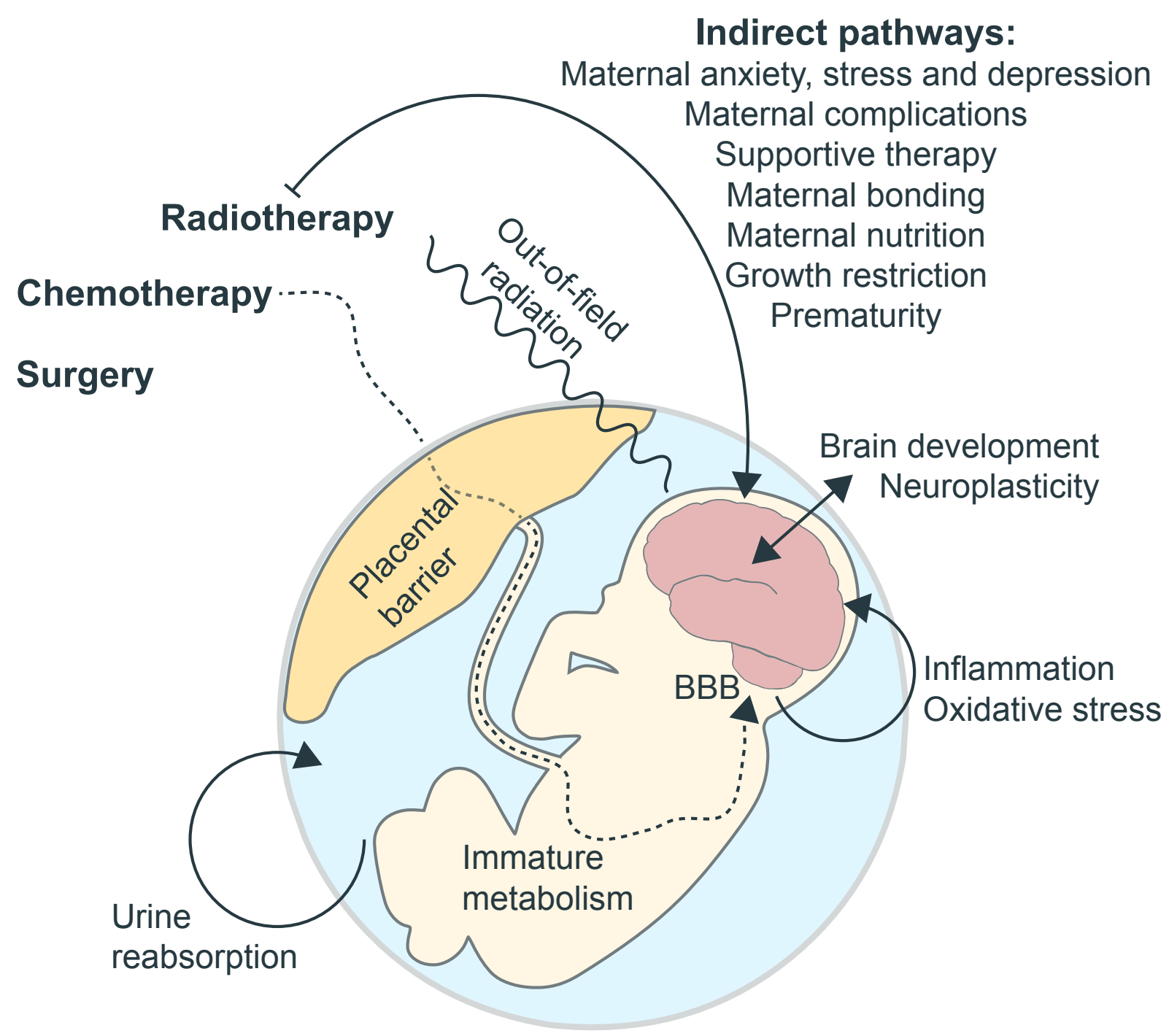

Figure 1.1 - Potential mechanisms of altered neurodevelopment after prenatal exposure to maternal cancer (therapy). This figure is based on work by Vercruysse et al. ${ }^{44}$. BBB: blood-brain barrier 


\subsection{The developing brain}

Compared to most other organs, the central nervous system has a prolonged development ${ }^{45}$. Moreover, as the fetal brain has a highly ordered development, the potential effect chemotherapy has on brain development will depend on its timing within the pregnancy ${ }^{16 ; 46 ; 47}$ (Figure 1.2). For instance, chemotherapy during the third to fifth months of pregnancy might interact with ongoing neuroblast proliferation and migration, while chemotherapy during the late stages of pregnancy might affect processes of cortical organization and early myelination ${ }^{16}$.

Many processes such as myelination, gyrification and synaptogenesis start in utero, but continue to develop postnatally ${ }^{45 ; 49}$. The white matter keeps maturing throughout childhood and adolescence, increasing both in volume, as well as continued microstructural development through myelination and axonal growth ${ }^{50-54}$. While in early childhood the GM still increases in volume, late childhood and adolescence is characterized by a decrease in GM thickness due to processes of synaptic pruning resulting in a higher specialization of the brain ${ }^{55-57}$

Recently, research has been investigating the importance of what happens during the first 1000 days of life for lifelong psychological functioning $45 ; 47 ; 58$. The rapid neurodevelopment in utero and during the first years of life make it a highly sensitive period for neurological impact, with potential long-lasting effects. On the other hand, this also gives the young brain an immense neuroplastic capacity, amplifying the benefits of early interventions ${ }^{59 ; 60}$.

As the brain develops, the impact of prenatal exposure on the cognitive development might only become apparent at older ages. For instance, executive functioning, has a prolonged development spanning from middle childhood until adulthood ${ }^{61}$. Furthermore, puberty has been identified as a vulnerable period for the development of psychiatric disorders ${ }^{62}$, which might have origins in early life events ${ }^{45 ; 63}$.

Long-term neurotoxic effects of numerous compounds have been described after prenatal exposure. Best known are fetal alcohol spectrum disorders ${ }^{64}$. This constitutes a group of neurocognitive impairments observed in children born to mothers with heavy alcohol consumption during pregnancy. Neurocognitive effects have also been observed after prenatal exposure to other compounds such as opiates and drug abuse ${ }^{65}$, lead, polychlorinated biphenyls and methylmercury ${ }^{66 ; 67}$, smoking ${ }^{68}$, licorize ${ }^{69}$, etc.

Besides the potential direct effects of cancer treatment during pregnancy on offspring neurodevelopment, numerous secondary mechanisms might also play a role ${ }^{44}$. The negative impact of both late-prematurity ${ }^{70-72}$ and fetal growth restriction ${ }^{72-74}$ on neurodevelopment have 


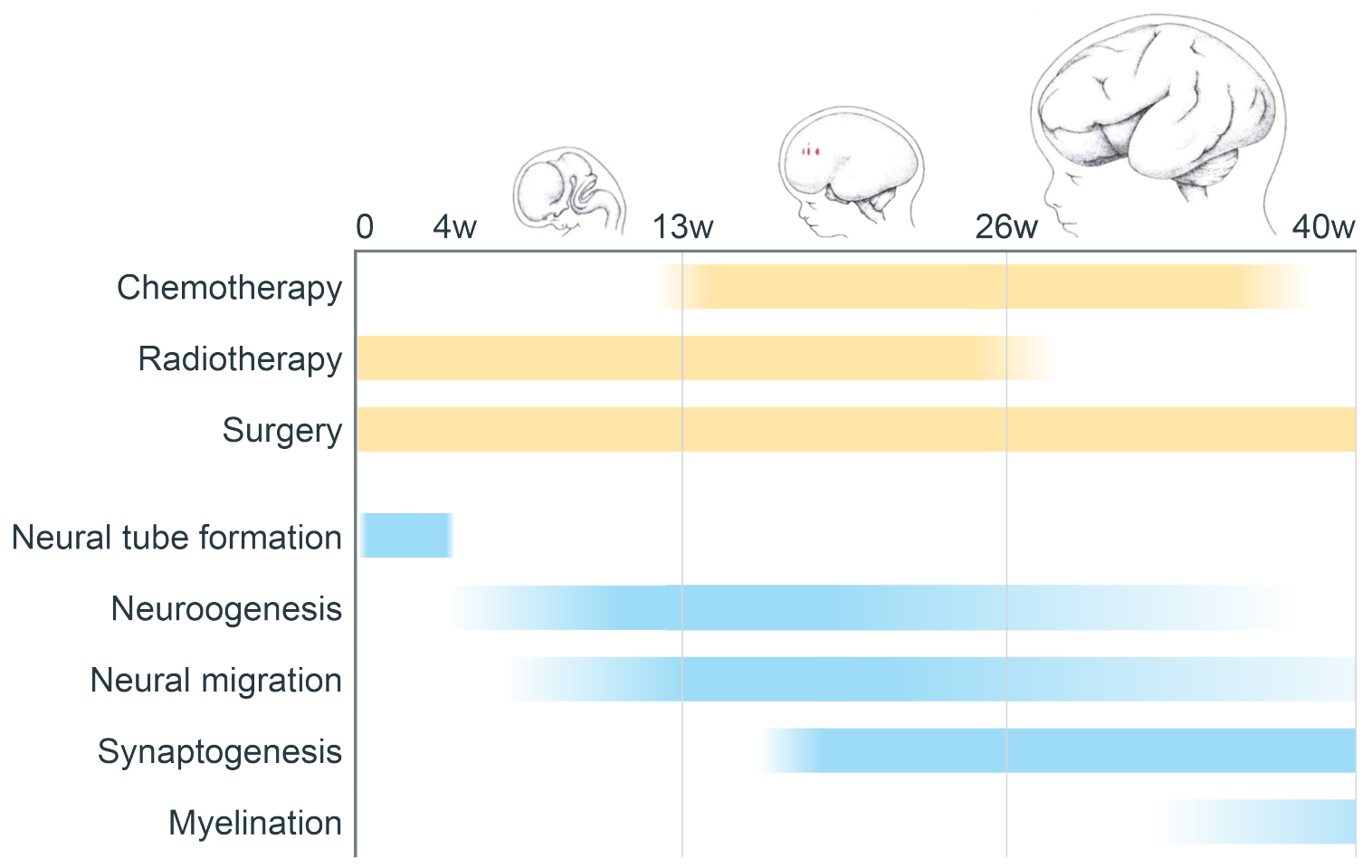

Figure 1.2 - Stages of prenatal brain development. Timing of different neurodevelopmental processes is depicted alongside possible time windows for different cancer treatments during pregnancy. This figure is based on work by Cowan et al. ${ }^{48}$, Scher et al. ${ }^{45}$, Jansen et al. ${ }^{16}$ and Amant et al. ${ }^{12}$

been described in the general population. Moreover, maternal stress ${ }^{59 ; 63 ; 75 ; 76}$, anxiety ${ }^{59 ; 77}$, depression ${ }^{59 ; 63 ; 75 ; 78 ; 79}$, anemia ${ }^{47}$ and malnutrition ${ }^{47 ; 59 ; 80}$ can affect the child's neurodevelopment. Furthermore, parental psychological coping and bonding with the child might impact postnatal neurodevelopment ${ }^{14 ; 81 ; 82}$.

\subsection{Multimodal neuroimaging}

The emergence of multimodal imaging techniques over the past decades, have provided a new window to non-invasively investigate the human brain. This has led to novel insights into the structure and function of the healthy ${ }^{51-55 ; 83-85}$ and impaired ${ }^{18 ; 63 ; 70 ; 71 ; 75 ; 75 ; 78 ; 79 ; 86}$ developing brain. Using multimodal magnetic resonance imaging (MRI), it is possible to probe multiple brain properties within one session. For instance, high-resolution T1-weighted imaging can be used to in detail describe the structural development of the brain ${ }^{87-89}$, multi-shell diffusion weighted imaging can investigate WM microstructure ${ }^{51 ; 90 ; 91}$ and resting-state functional $\mathrm{MRI}^{92 ; 93}$ can be used to probe co-activation patterns in the brain. On the other hand, Event-related potentials can non-invasively measure the electrophysiological response of the brain to psychological tasks with a high temporal resolution ${ }^{94}$. This diversity in neuroimaging techniques makes it a very strong toolkit in assessing the neural substrate of prenatal exposure to cancer treatment. In chapter 3 , 
we will discuss in greater detail the imaging techniques applied throughout this dissertation.

\subsection{Conclusions}

In summary, while there is strong evidence on the short-term fetal safety of cancer treatment during pregnancy, subtle cognitive effects, the lack of long-term data and the multitude of potential pathways through which this treatment could affect neurodevelopment necessitates robust long-term follow-up data. In addition to neuropsychological testing and questionnaires, emerging multimodal neuroimaging techniques could help to establish the neurodevelopmental risks of antenatal exposure to cancer treatment. 


\section{CHAPTER}

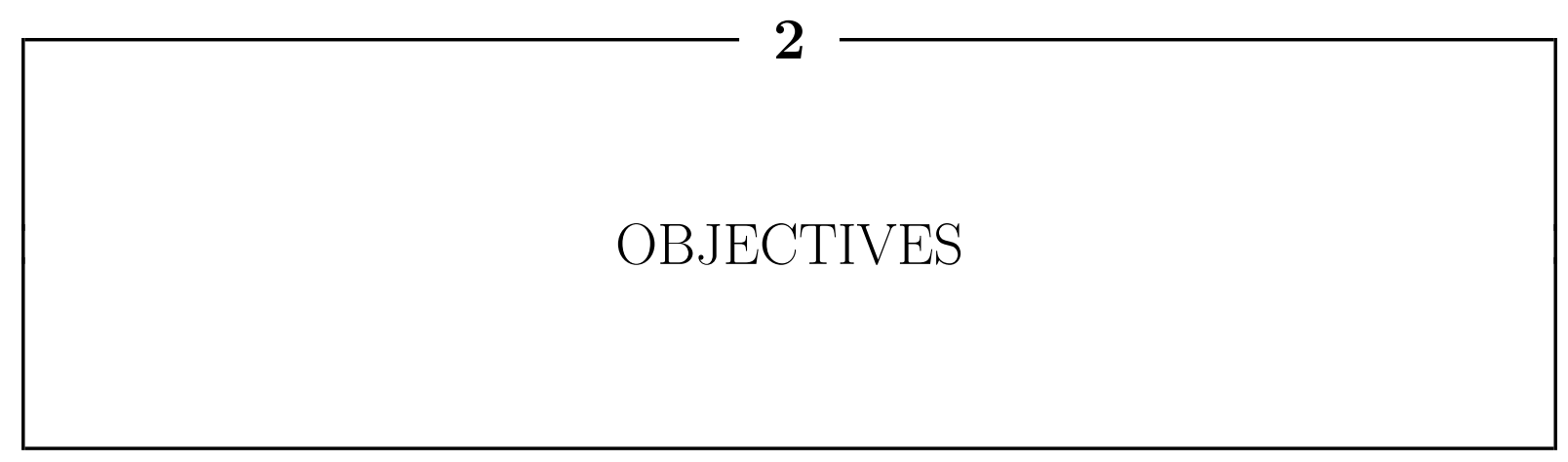

The main aim of this $\mathrm{PhD}$ research is to assess the long-term safety of prenatal exposure to maternal cancer and cancer treatment with respect to the neurocognitive development. In particular, we want to address following research questions:

1. How does chemotherapy affect the brain? Chemotherapy is by far the most commonly administered systemic treatment for cancer during pregnancy ${ }^{2}$. However, before investigating the impact of prenatal exposure to cancer treatment on brain development, we aimed to increase our insights on the pathways through which intravenously administered chemotherapy affects the brain. Chemotherapy has been associated with long-term neurocognitive problems in a multitude of cancer populations ${ }^{18 ; 20 ; 21 ; 38}$. Ahles and colleagues ${ }^{20}$ recently proposed a model in which treatment interacts with a multitude of factors, including genetic, physiologic, psychological and socio-demographic elements, resulting in the so-called 'Chemobrain'. In chapter 4 we investigate the effects of chemotherapy on brain volume in non-pregnant patients with breast cancer and examine the interaction of these effects with aging and menopause.

2. Does cancer and its treatment during pregnancy affect neurodevelopment of the child? Multimodal neuroimaging techniques are used to study the multifaceted nature of brain development and the potential impact prenatal cancer treatment. In chapter 5, we will use event-related potentials to investigate the impact of prenatal chemotherapy exposure on the development of executive functions. In chapter 6 , we will 
use multimodal MRI techniques to investigate the impact of prenatal exposure to cancer treatment on the developing brain structure and function.

3. Which treatment-related and secondary factors play a role in the child's neurodevelopment after maternal cancer during pregnancy? Cancer in pregnancy is a heterogeneous diagnosis with different pathologies and treatments, occurring at different stages of pregnancy resulting in a different treatment plan. These differences might have distinct effects on the foetal neurodevelopment. Moreover, a multitude of secondary factors might affect brain development as well ${ }^{44}$. Late prematurity, in particular, often occurs in this population and has been associated with worse cognitive outcome ${ }^{6}$. In both chapters 5 and 6 , we will try to distinguish effects of cancer in pregnancy from the effect of late-prematurity on the development of executive functions. Moreover, in chapter 6 , we will conduct exploratory analyses to find potential risk factors of cancer during pregnancy that affect structural and functional neurodevelopment of the brain.

Next, in chapter 3, we will outline the methodology used throughout this $\mathrm{PhD}$ project. Then, chapters 4 to 6 will discuss each aforementioned study in detail. Finally, in chapter 7 , we will provide a general overview and discussion on the insights from the different studies. 


\title{
CHAPTER
}

\author{
3
}

\section{METHODOLOGY}

The following sections describe key aspects of the used methodology throughout this dissertation. Figure 3.1 shows the acquired neuroimaging modalities and how they are employed to investigate the brain and its development.

\subsection{Participants}

In chapter 4, we will investigate the effects of chemotherapy on brain volume in patients with breast cancer. For this we will group 3D T1-weighted MRI data from different longitudinal datasets of pre- and post-menopausal breast cancer patients, acquired by Deprez et al ${ }^{23 ; 28-30}$. Participants underwent MRI scanning between 2008 and 2014, before start of chemo- and/or radiotherapy and after completion of chemo- and/or radiotherapy, using same scanner and protocol at UZ Leuven. In total, 180 women participated: 121 patients who were diagnosed with early-stage breast cancer, of whom 72 received chemotherapy, and 59 healthy controls. Young participants $(n=128)$ were pre-menopausal at start of the study and younger than 52 years. Older participants $(n=52)$ were post-menopausal and older than 60 at start of the study. While previous studies in these cohorts already analysed the functional ${ }^{23}$ and diffusion $\mathrm{MRI}^{28-30}$, the T1-weighted images had not yet been studied.

In chapters 5 and 6 , we respectively use event-related potentials and multimodal MRI to study the 


\section{Magnetic resonance Imaging}
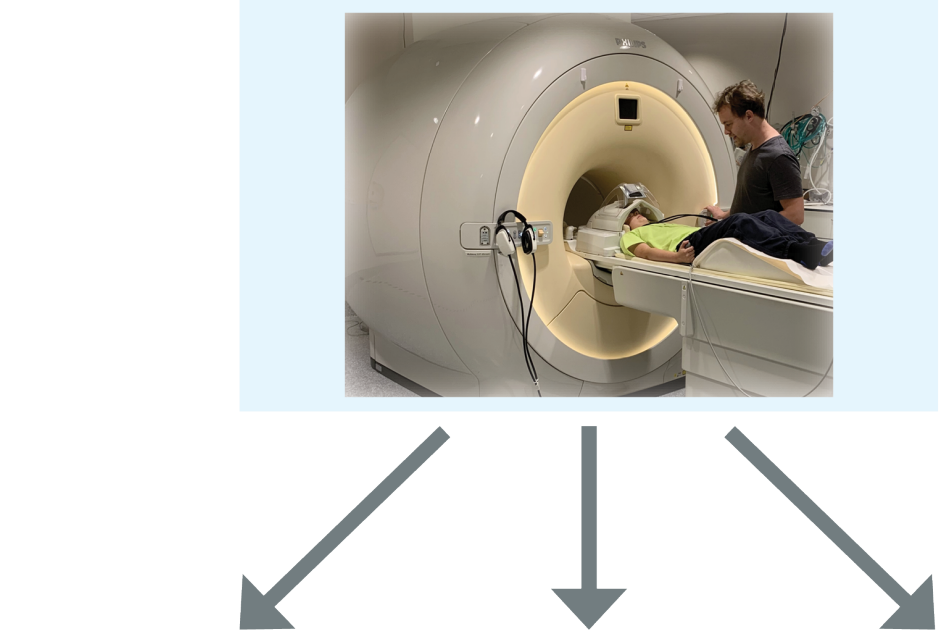

\section{T1-weighted}
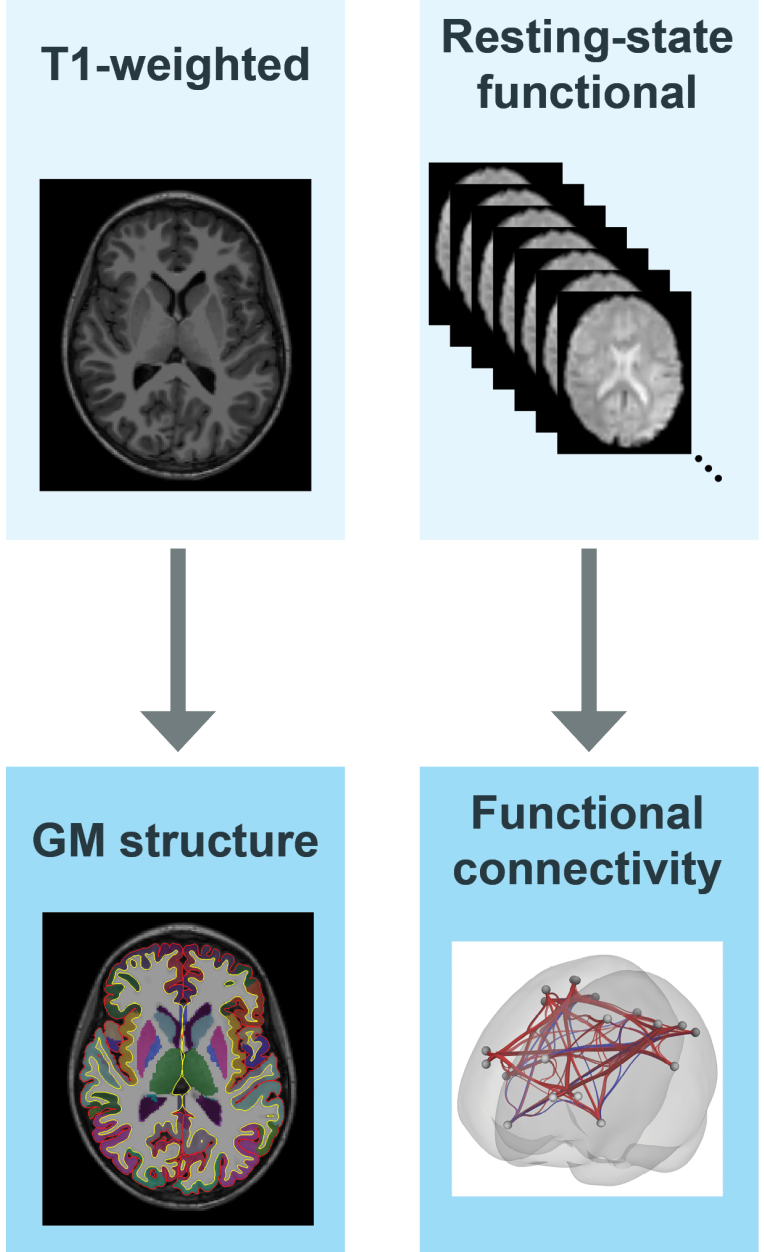

\section{Multi-shell diffusion}
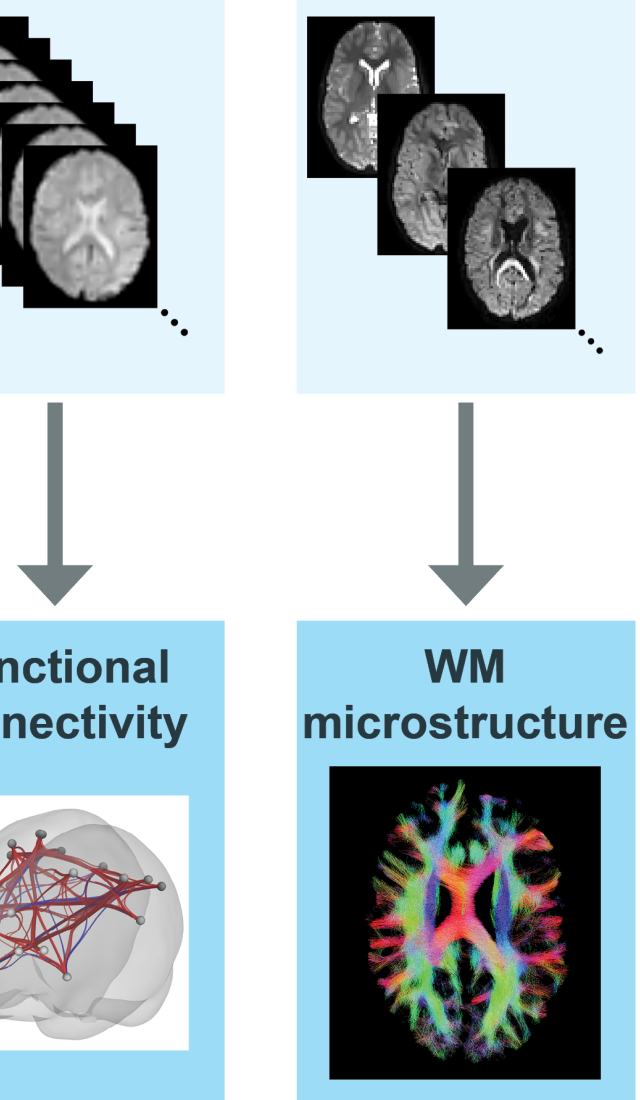

\section{Event-related potentials}
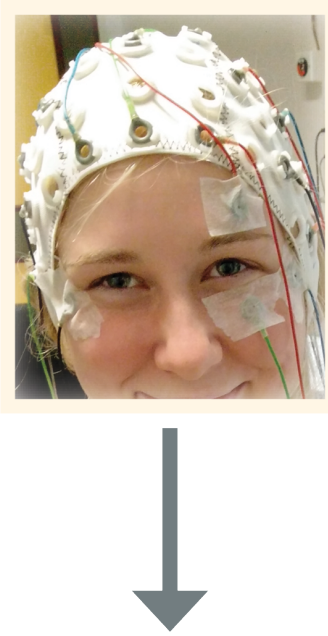

\section{EEG + task}
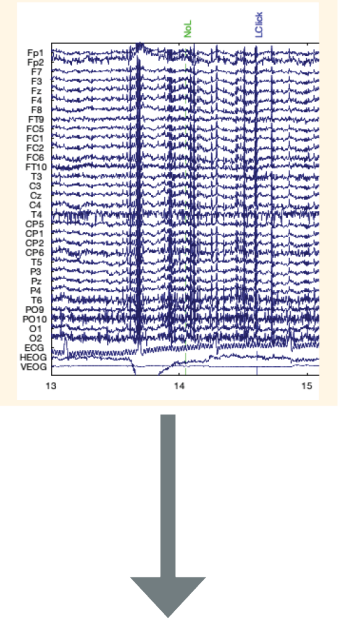

Task-related neural activity

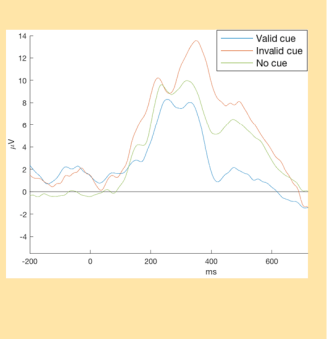

Figure 3.1 - Overview of neuromaging modalities used throughout this dissertation. The different modalities are displayed along their main usage. EEG: electroencephalography. GM: grey matter WM: white matter. 
effects of prenatal exposure to maternal cancer and its therapy on brain development. For this, two groups of children are recruited: (1) a study group of children born to cancer-complicated pregnancies and (2) a matched control group. All children are tested at the age of nine years. Control children are matched to study group children in a 1:1 ratio regarding age, sex and gestational age at birth (GA). Exclusion criteria are major neonatal complications with a potential impact on neurodevelopment, such as hypertension, pre-eclampsia, gestational diabetes, infection/sepsis and twin-to-twin transfusion. All children are recruited within the Belgian prospective follow-up study of children born after cancer in pregnancy, which is part of an international INCIP study. In total, ERP data on 20 children with prenatal chemotherapy exposure, with equal numbers of matched controls, were recruited between 2010 and 2018, and included in the analyses of chapter 5 . Similarly, MRI data of 42 children born after cancer-complicated pregnancies and matched controls, acquired between 2015 and 2020, was included for the analyses of chapter 6 . Next to the neuroimaging follow-up of this study, children underwent extensive psychological testing and the clinical history is registered in the international INCIP registry.

\subsection{Event-related potentials}

Event-related potentials (ERP) is a technique where neural activity in response to a given stimulus or task is measured with an electroencephalogram (EEG) ${ }^{94}$. As neural activity is measured through resulting electrical potentials at the scalp it has a much lower spatial resolution compared to task-based functional MRI. However, whereas functional MRI has a temporal resolution of 1-3 seconds, ERPs operate within a millisecond range. This allows ERPs to identify temporal subprocesses of a neural response to a given stimulus. In chapter 5, we will use ERPs to asses the effect of maternal chemotherapy during pregnancy on the development of executive functioning.

Two tasks will be used to investigate different aspects of executive functioning (Figure 3.2). First, a Go/Nogo task will be used to asses response inhibition. During this task, participants are instructed to press a button whenever a frequent Go-stimulus appears but not press when the occasional Nogo-stimulus is shown. The frontal N2 peak in the resulting ERP is associated with neural processes of conflict monitoring, whereas the central to parietal P3 peak is more closely associated with response inhibition itself ${ }^{95-97}$ (Figure 3.3).

Second, a Posner task will be used to assess spatial attention ${ }^{98 ; 99}$. During this task, a stimulus appears on either the left or right side of the screen, and participants are asked to press correspondingly with a left or right button click. Often a cue is presented in advance, indicating 
Go/Nogo

$80 \%$ Go

$20 \%$ Nogo
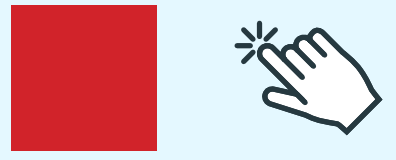

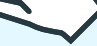

(1)

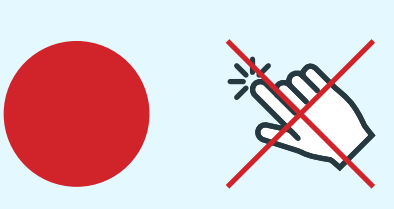

\section{Posner Paradigm}

Exogenous cue (1/4 false)
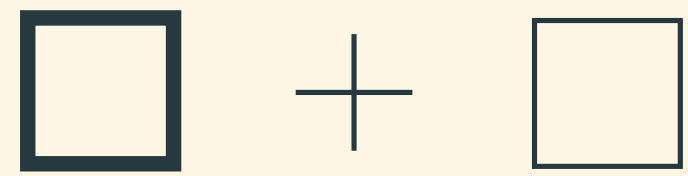

Or

Endogenous cue (1/4 false)

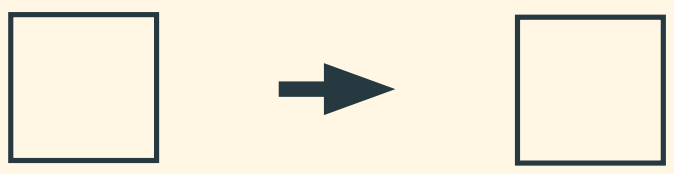

Task
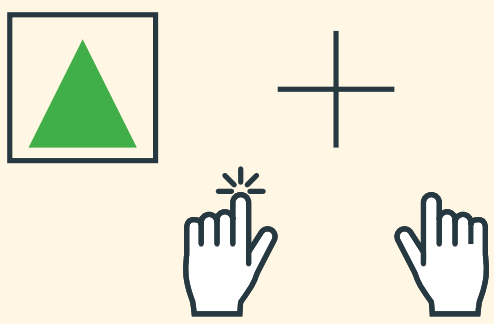

Figure 3.2 - ERP paradigms. ERP paradigms used in the work of chapter 5 

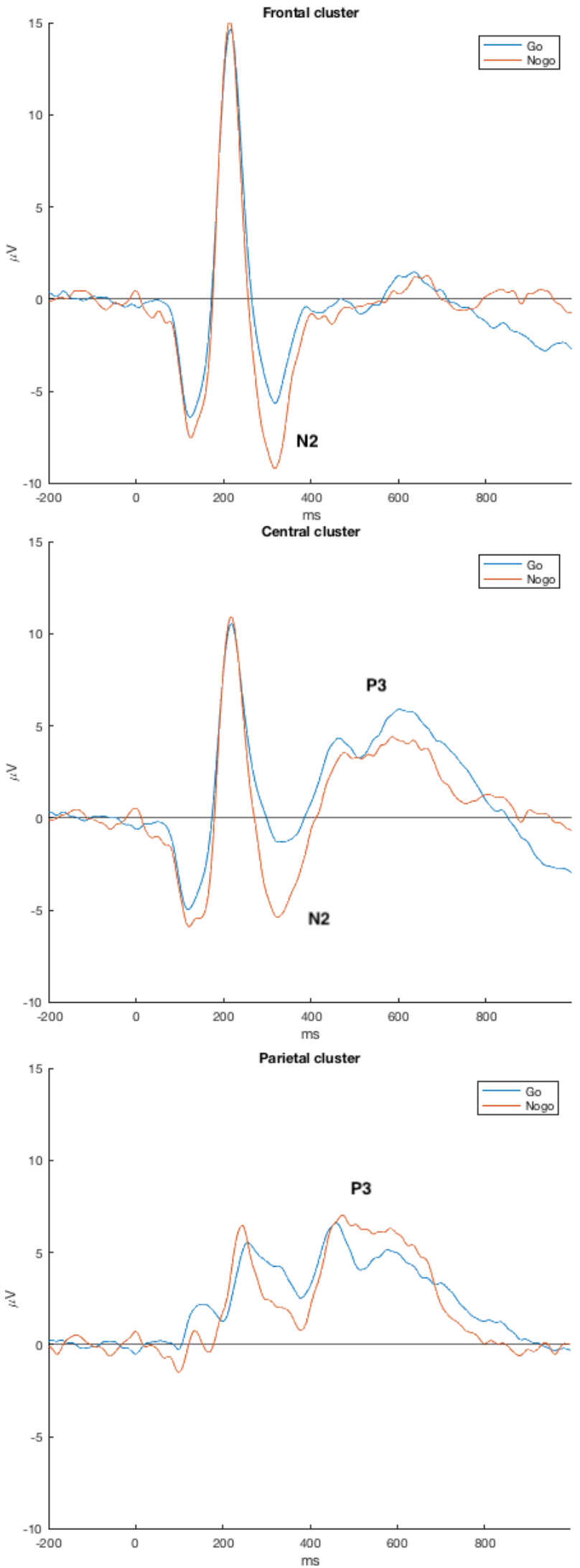

Figure 3.3 - Go/Nogo grand average potentials. Grand average ERPs of the work in chapter 5 are visualized with their key features annotated 
where the stimulus will appear. This cue can be either endogenous, in our case an arrow in the middle of the screen which instructs the participant to shift his/her attention, or exogenous, in our case the left or right box becoming thicker which automatically draws the attention of the participant to that side of the screen. However, sometimes a false cue is given, after which the participant has to refocus his/her attention to the correct stimulus location. The most important ERP features for this paradigm are the parietal to occipital P3 and positive slow wave, which are associated with response selection and attentional set updating ${ }^{100 ; 101}$ (Figure 3.4). 

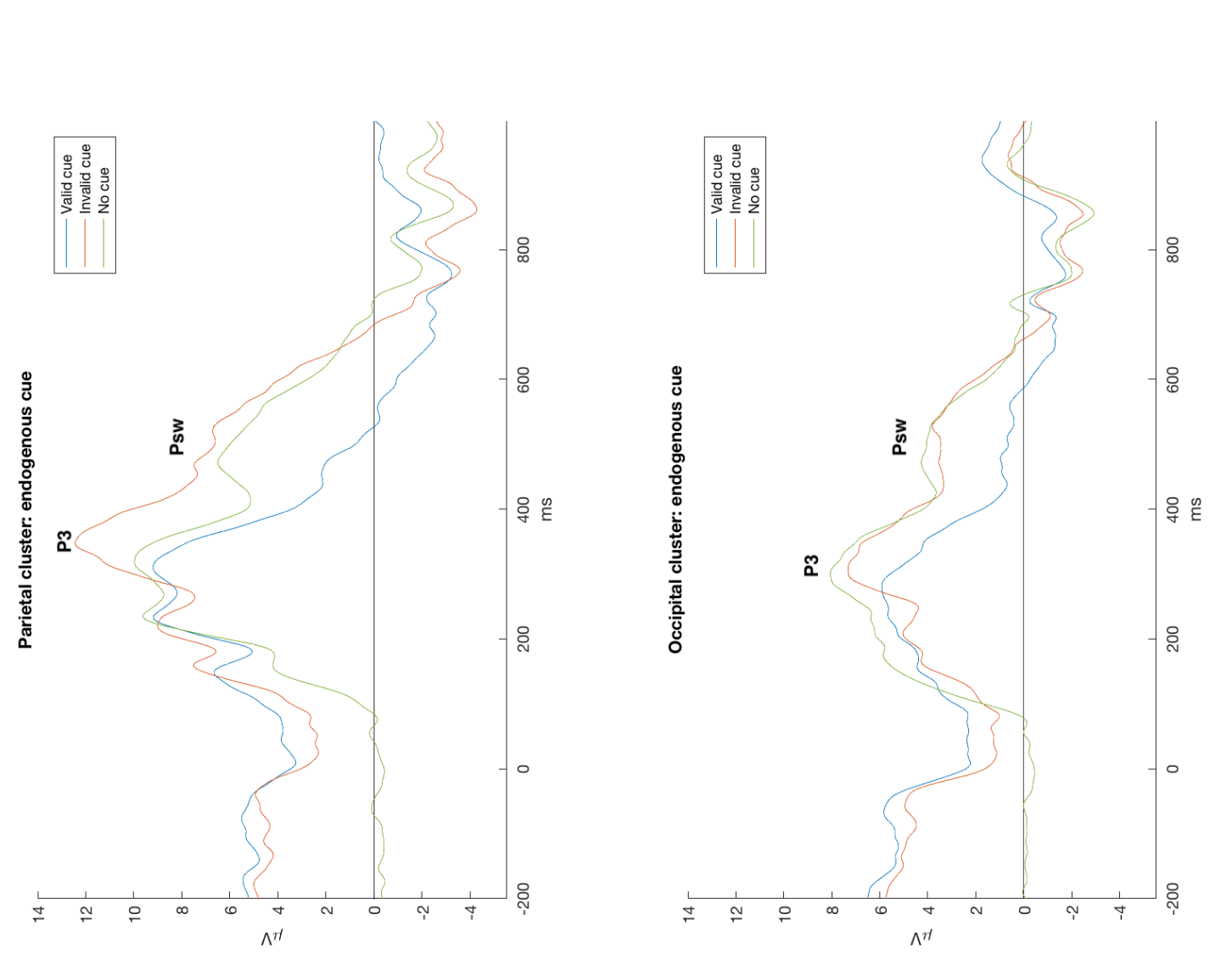

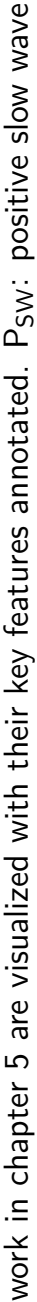
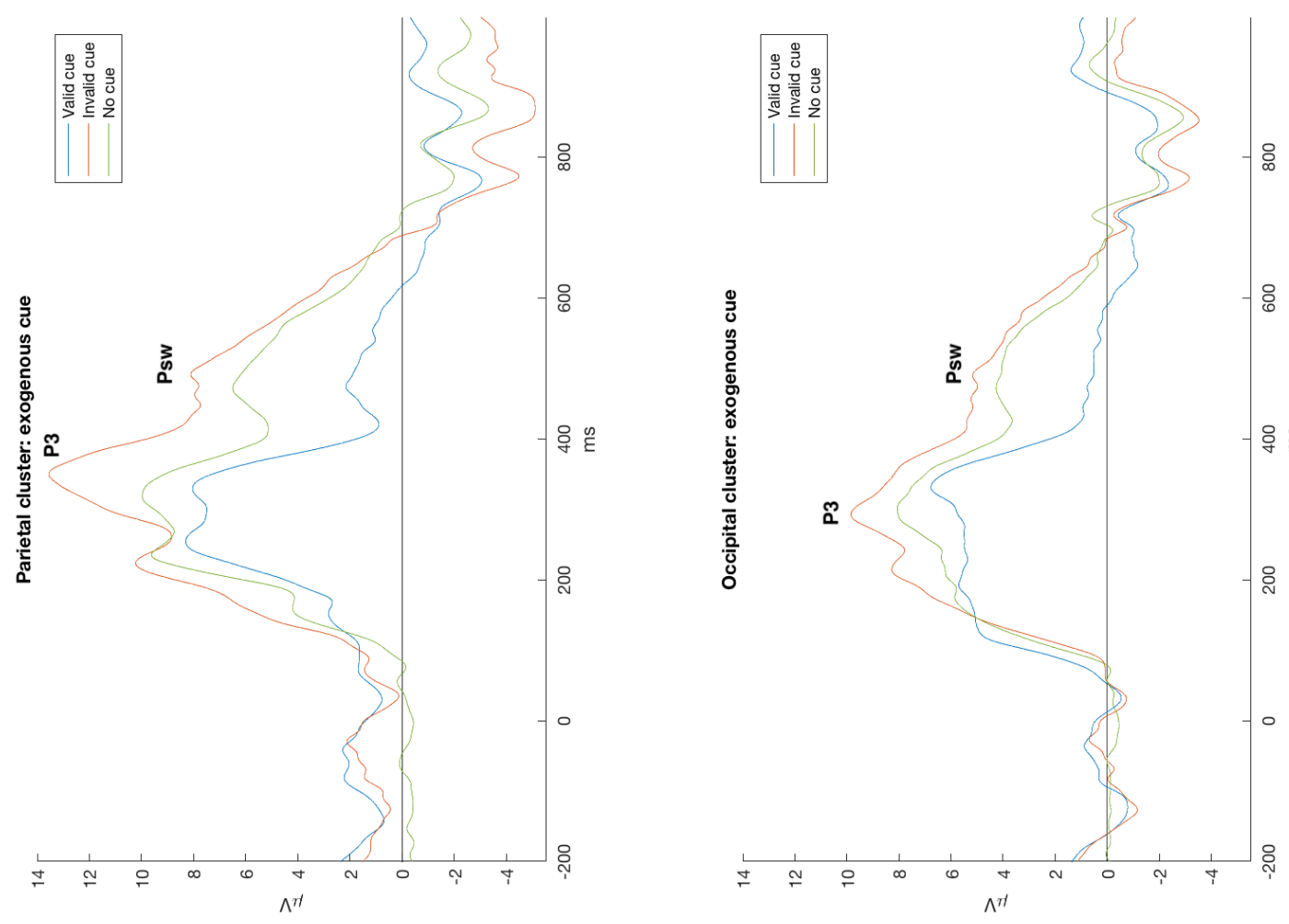

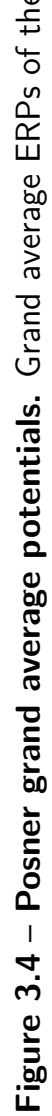




\subsection{Magnetic resonance imaging}

Magnetic resonance imaging (MRI) uses radiofrequent (RF) pulses to excite hydrogen protons out of a large static magnetic field, called the B0 field. This excitement is performed at the resonant Lamor frequency $(\omega), i / e$. the rate at wich the hydrogen protons spin around their magnetic axis:

$$
\omega=\gamma B_{0}
$$

where $\gamma$ is the gyromagnetic ratio which for hydrogen is $42.58 \mathrm{MHz} / \mathrm{T}$. In other words, under a 3 Tesla B0 field, which is a common MR field strength for neuroimaging, hydrogen will precess around its magnetic axis at about $128 \mathrm{MHz}$, being the resonant frequency that will then be used to excite the protons. The rate at which the spins of the excited protons realign with the B0 field can be characterized by the longitudinal $\mathrm{T} 1$ and tranverse $\mathrm{T} 2$ relaxation times, which are highly dependent on the capacity of the surrounding tissue to absorb this energy. A T2* contrast can be used to capture small inhomogeneities in the Lamor frequency, by looking at dephasing of the spins.

Different tissues exhibit different $\mathrm{T} 1, \mathrm{~T} 2$ and $\mathrm{T} 2 *$ relaxation images. For instance, water, e.g. in the cerebral spinal fluid, has long T1 and T2 relaxation times as the majority of hydrogen atoms are in $\mathrm{H}_{2} \mathrm{O}$, giving it few possibilities transferring it energy, resulting in water showing bright on T2-weighted images and dark on T1-weighted images. Fat, on the other hand, exhibits very short $\mathrm{T} 1$ and $\mathrm{T} 2$ relaxation times as many hydrogen atoms are involved in $-\mathrm{CH}_{2}$ - bonds giving many possibilities for transferring energy. In the brain, white matter, as compared to grey matter, has slightly higher T1 and T2 relaxation times. By combining T1, T2 and T2* contrasts, MRI can be used to reveal different contrasts in the brain between tissues and measure different properties of these tissues, making it an ideal tool to study the multifaceted nature of neurodevelopment ${ }^{102}$.

This way MRI offers a unique, non-invasive and safe tool to study the brain, without exposing participants to ionizing irradiation ${ }^{103}$. However, acquiring high-quality MRI data from children can be challenging as in-scanner movement can impair data quality and bias results from analyses ${ }^{56 ; 104 ; 105}$. In this study, we have given particular attention to age-adequately instructing and familiarizing children with the MRI environment. Additionally, children are shown a movie during scanning as distraction. Next, state-of-the-art image processing techniques were applied to minimize effects of motion in the image analyses ${ }^{106-109}$. For instance, fmriprep ${ }^{108}$ was used to preprocess the fMRI images, including steps of spatio-temporal filtering ${ }^{110}$, slice-time correction $^{111}$ and regression of motion artifacts using independent component analysis ${ }^{107}$. For diffusion-weighted images, motion between slices and volumes, as well as motion-induced signal drop-out were simultaneously corrected, using spherical harmonics and radial decomposition 
(SHARD) signal reconstruction ${ }^{109}$. Finally, at multiple stages of image processing we performed qualitative and quantitative evaluation of image quality ${ }^{109 ; 112}$.

For the study detailed in chapter 6 , children underwent a half-hour MR scanning protocol. The following subsections will explain in greater details how different image analysis techniques are applied on different image contrasts to extract information on the structural and functional development of the brain.

\subsubsection{Morphometry}

Morphometry, originating from the greek words morphe (form) and metria (measurement) ${ }^{113}$, is the study of shape and size. High resolution T1-weighted MRI has made it possible to noninvasively acquire anatomically detailed images of the brain, with a high contrast between WM and GM, in just a couple of minutes. This way, we can study local and global variations in WM and GM density, volume and shape in both healthy and impaired neurodevelopment ${ }^{55 ; 56 ; 114}$.

Three main families of morphometrical methods can be distinguished within the field of neuroimaging: deformation-based (DBM), voxel-based (VBM) and surface-based (SBM) morphometry ${ }^{87-89 ; 115 ; 116}$. In DBM, information on volume is extracted from the nonlinear registration of the original image to a common template through the Jacobian of the deformation matrix ${ }^{115}$. Unmodulated VBM relies on tissue probability maps (TPM) derived from segmenting the image into WM, GM and $\mathrm{CSF}^{88 ; 89}$. The probability of each voxel belonging to one of these three tissues can then be interpreted as a density of those tissues in that voxel. Modulated VBM, multiplies the Jacobian and TPM to generate measures of tissue-specific volume ${ }^{87-89}$. Finally, in SBM the pial and GM-WM surfaces are reconstructed ${ }^{116}$. This allows us to study both the GM thickness, as well as various other parameters of cortical shape.

\subsubsection{Resting-state functional MRI}

In functional MRI (fMRI), whole-brain 2D T2*-weighted images are repeatedly acquired, each taking only 1-3 seconds. When the brain consumes oxygen, diamagnetic oxygenated hemoglobin is metabolized into paramagnetic deoxygenated hemoglobin. During functional activation of a certain brain region, more blood is supplied to this region through vasodilization. This dynamic process is subsequently observed in the functional images as the blood oxygenation level dependent (BOLD) signal ${ }^{117}$.

However, since BOLD fMRI assesses neural activity through a hemodynamic response, nonneuronal physiological activity (e.g. changes in heart beat) can influence the signal as well. 
Moreover, since multiple images have to be acquired at short time intervals in order to capture this dynamic process, resulting in lower image resolution, motion and partial-volume effects can further affect these images. For this reason, it is of high importance to address as good as possible these different sources of bias and noise ${ }^{118}$.

In task-based fMRI experiments, imaging is performed while the participant executes a certain task. Both simple tasks (e.g. finger tapping) as well as more complicated tasks (e.g. a multi-tasking experiment ${ }^{119}$ ) can be used. Typically, tasks are interleaved by blocks of rest. By contrasting the BOLD signal during the task to the signal during the resting condition, patterns of regional activation and deactivation during the task can be observed.

More recently, resting-state $\mathrm{fMRI}$ ( $\mathrm{rs}-\mathrm{fMRI}$ ) has been introduced as a method for observing spontaneous fluctuations in the BOLD signal during rest. Unlike task-based fMRI, neuronal activity during resting-state cannot be inferred by contrasting two conditions. Instead, statistical methods, such as independent component analysis (ICA) or correlations in BOLD activity between regions, are used to observe patterns of spontaneous co-activation between brain regions. This way different functional networks of concurrent activation such as the default-mode, auditory, salience or dorsal attention network can simultaneously be observed, analyzed and compared $^{92 ; 93 ; 117 ; 120}$.

\subsubsection{Diffusion-weighted imaging}

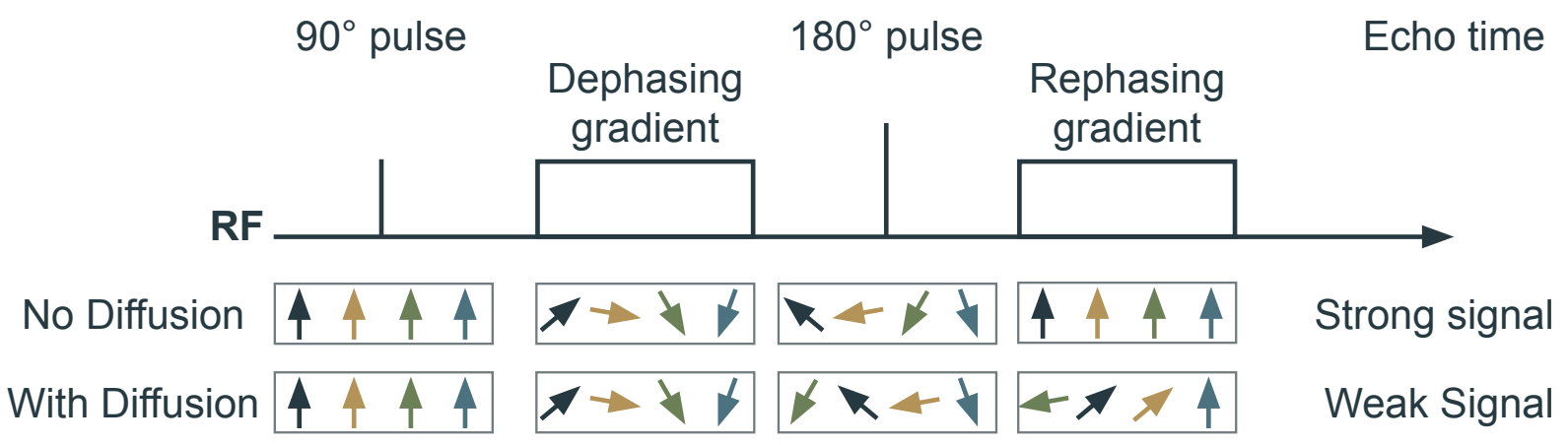

Figure 3.5 - Principles of diffusion MRI.

Diffusion-weighted MRI uses two gradients interspersed with a $180^{\circ}$ refocusing pulse to capture capture diffusion of the hydrogen protons in a particular direction (Figure 3.5). The first gradient dephases the spins of the protons along the axis of the gradient. Through the $180^{\circ}$ refocusing pulse and the rephasing gradient this dephasing would be undone. However, due to Brownian motion, hydrogen particles will move from their original position along the gradient axis and thus a signal loss will be induced. 
Biological tissue can hinder or restrict movement of water molecules (Figure 3.6). This way, diffusion imaging can be used as a tool to, although indirectly, investigate microstructural properties. The mean diffusivity (MD), also known as the apparent diffusion coefficient (ADC), of a voxel can be reliably estimated with only a limited number of directions, which is often used in clinical practice in a variety of application such as identification and characterization of tumors ${ }^{90}$.

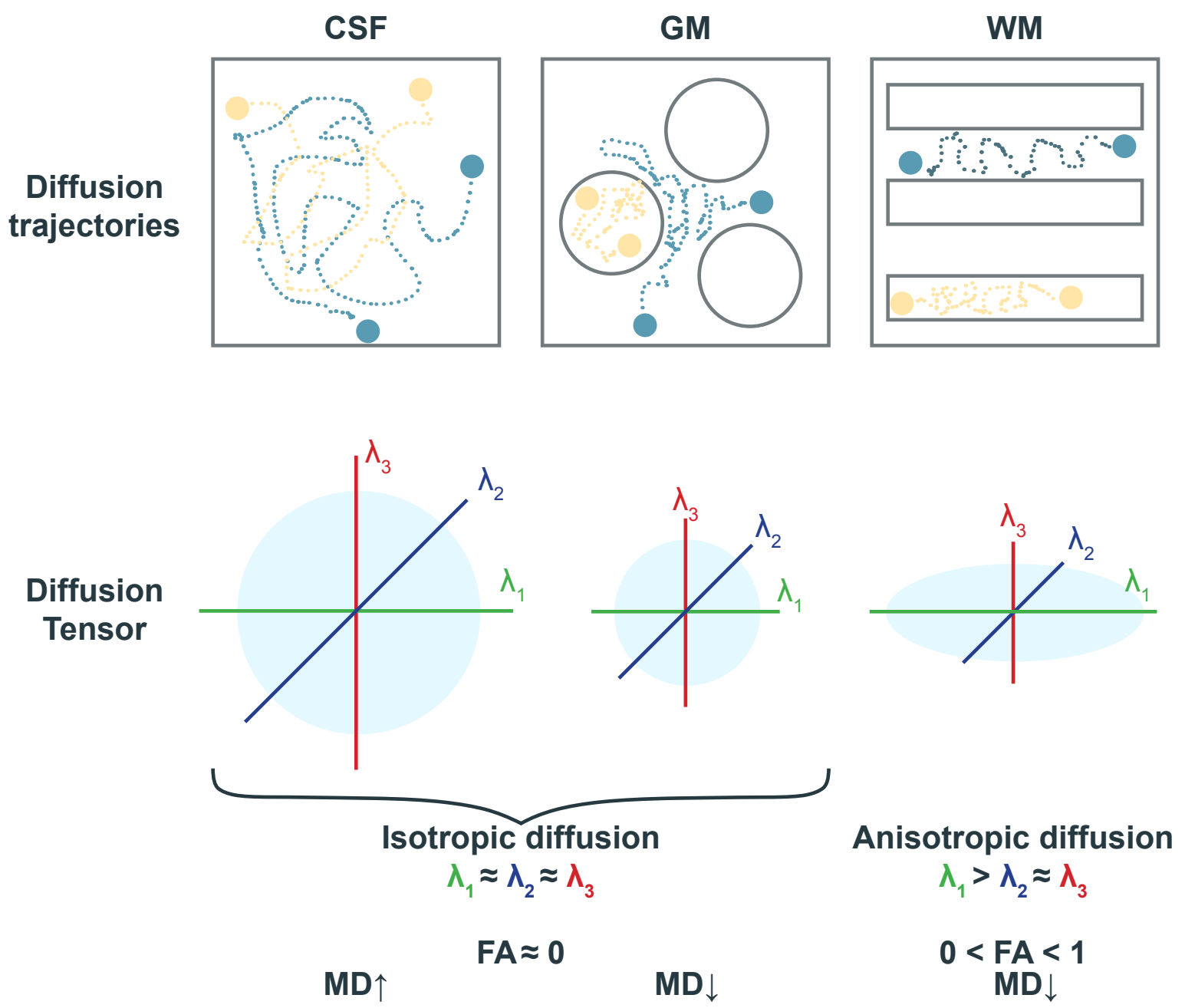

Figure 3.6 - The diffusion signal in different brain tissues.

More recently, techniques like diffusion tensor imaging (DTI) and high angular resolution diffusion imaging (HARDI) have enabled us to probe at the WM microstructure by applying diffusion imaging at high diffusion sensitizing gradients, termed the b-value, and at a high angular resolution (using 30 or more directions for each b-value) ${ }^{91 ; 121}$. The WM fiber pathways, containing bundles of myelinated axons, restrict the brownian motion of water in a highly directional fashion (Figure 3.6). Intra-axonal water molecules are restricted by the axonal boundaries, making it relatively easy to move along the axon, but difficult to move perpendicular to it. Extra-axonal water molecules on the other hand, are hindered by the microstructural 
content, but still can move past it, although less rapidly. This anisotropic property of the diffusion signal can then be used to study microstructure of the underlying WM pathways.

Diffusion tensor imaging is the earliest and still most clinically used model that applies diffusion MRI to probe WM microstructure ${ }^{91}$. This model uses a three-dimensional tensor to model the anisotropic diffusion in a spheroid. MD can be calculated within this model as the mean of the three eigenvalues of this tensor. The anisotropy in the diffusion tensor is typically quantified using the formula of fractional anisotropy (FA):

$$
F A=\sqrt{\frac{1}{2}} \sqrt{\frac{\left(\lambda_{1}-M D\right)^{2}+\left(\lambda_{2}-M D\right)^{2}+\left(\lambda_{3}-M D\right)^{2}}{M D}}
$$

With $\lambda_{1-3}$ the eigenvalues of the tensor. Intuitively, one can see that FA=1 in the case of perfect anisotropy, i.e. $\lambda_{1}>0$ and $\lambda_{2}=\lambda_{3}=0$, and 0 in case of isotropy, i.e. $\lambda_{1}=\lambda_{2}=\lambda_{3}$, as for example in water. While DTI can be used to visualize WM pathways and its parameters can be used to investigate WM microstructure, it has some shortcomings. First, this model can only accurately describe the diffusion signal within WM when only one fibre population is present in a voxel, and this population does not change direction within this voxel. Unfortunately, with current voxel sizes, up to $90 \%$ of voxels are estimated to have one or more fanning, bending or crossing fibers ${ }^{122}$. Second, the DTI model is unable to differentiate between hindered and restricted diffusion, coming from different tissue compartment within the WM.

Recent models, such as Neurite Orientation Dispersion and Density Imaging (NODDI) ${ }^{123}$ and fixel-based analysis (FBA ${ }^{124 ; 125}$, uses multiple b-values to respectively distinguish signals from intra- and extra-axonal compartments in the WM or disentangle signals coming from crossing fiber populations. Compared to DTI, these methods have proven to be more sensitive biomarkers for microstructural WM development ${ }^{51 ; 53}$. Whereas NODDI applies a biophysical model to characterize CSF, extracellular and intracellular compartments ${ }^{123}$, FBA extracts information from higher-order fibre orientation distributions (FOD) ${ }^{124 ; 125}$, generated using mathematical models such as constrained spherical deconvolution $(C S D)^{126}$. This way, FBA poses far less assumptions on the underlying signal. Throughout the work of chapter 6 we will use FBA as our model for investigating WM development in children born after cancer-complicated pregnancies.

\subsection{Graph theory}

The brain can be viewed as a large network. Billions of neurons ${ }^{127}$ in the GM process and share information through a network of short an longer connections. Using MRI, such a brain network can be constructed using measures of structural or functional connectivity. Then graph theory 


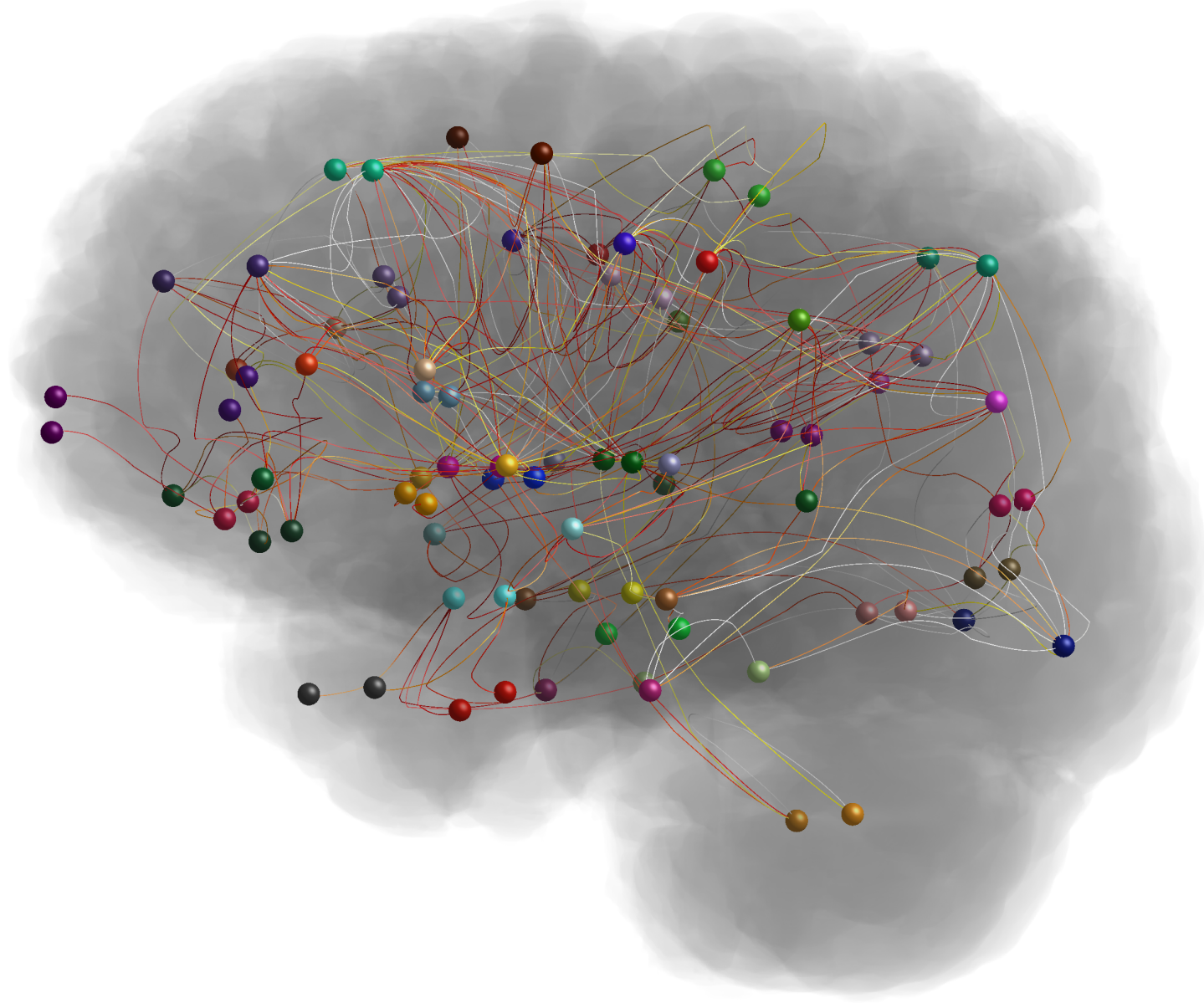

Figure 3.7 - Visualization of a brain connectome. This connectome was created using CSD tractography ${ }^{126}$ and parcellation of the brain using freesurfer ${ }^{129}$ as detailed in chapter 8 . Connections are visualized along their mean pathway, and color-coded based on the number of SIFT2-reweighted connections.

can be applied to calculate various properties of this network.

To understand this principle we can compare such network to a road map. When a car accident happens on an important highway during rush hour, it will induce huge traffic jams. On the other hand, the same accident on a rural road will not highly affect traffic.

Similarly, the impact of a brain injury on the cognitive outcome depends on its location and extent. In contrast to the adult brain, the young brain has a large neuroplastic capacity. As a result, an impact in the brain might be partially compensated, as other pathways take over $^{47 ; 59 ; 128}$.

In order to build a connectome (Figure 3.7) nodes and edges have to be defined. In neuroimaging, nodes are typically meaningful anatomical structures, originating from a parcellation of the GM. Edges quantify the amount of connectivity between these regions, as can be defined using WM tractography ${ }^{130}$ on diffusion-weighted images ${ }^{131 ; 132}$ or by functional co-activation in the rs-fMRI 
signal $^{92}$. Once a connectome is constructed, diffferent mathematical features concerning the organization of the network can be calculated on a whole-brain, cluster, node or edge level ${ }^{92}$.

Graph theory provides a general framework to study organization and efficiency of this brain network $^{92}$. Moreover, the universal nature of this technique allows it to be applied not only to networks based on functional or diffusion-weighted MRI, but also based on co-activation in the EEG $^{133}$ signal or even similarity in morphometrical features ${ }^{134}$. 


\section{CHAPTER}

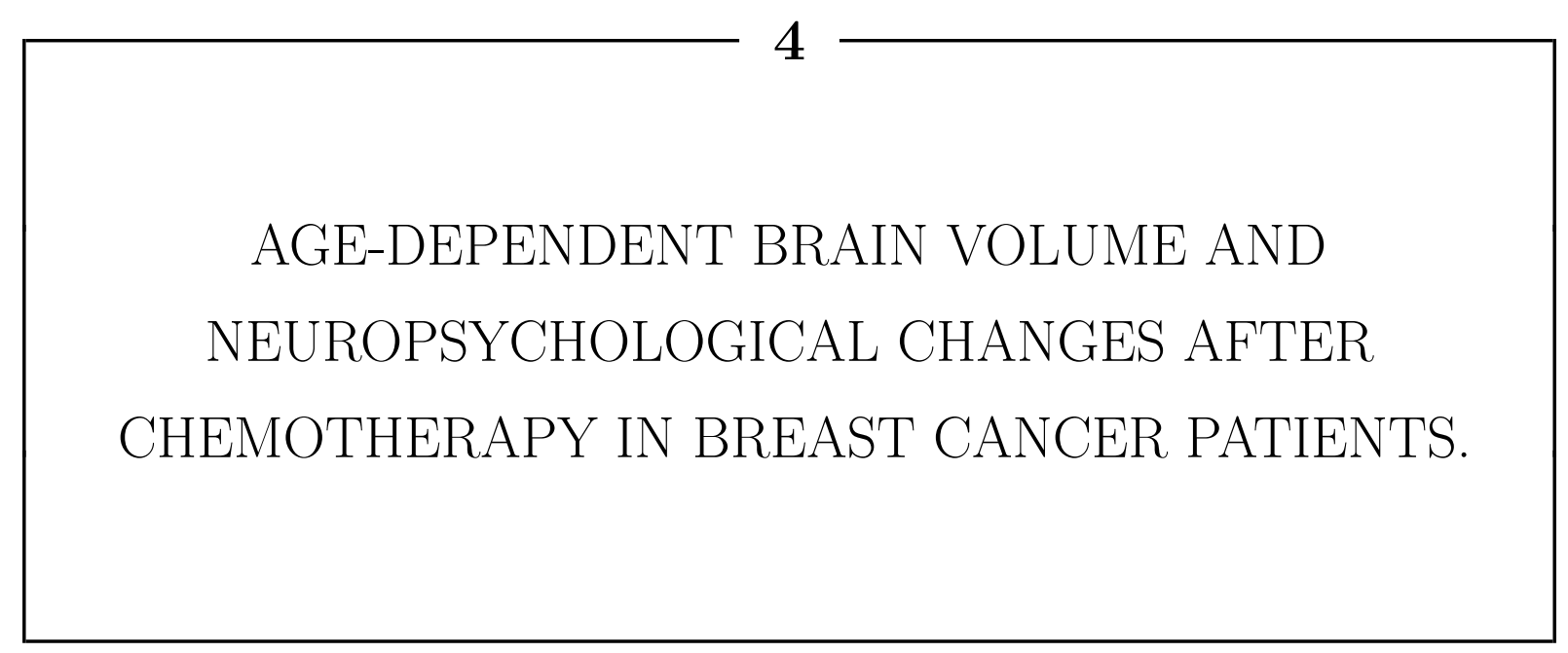

This chapter has been published as:

Blommaert J., Schroyen G., Vandenbulcke M., Radwan A., Smeets A., Peeters R., Sleurs C., Neven P., Wildiers H., Amant F., Sunaert S. and Deprez S. (2019). Age-dependent brain volume and neuropsychological changes after chemotherapy in breast cancer patients.

Human Brain Mapping, 40 (17), 4994- 5010. doi: 10.1002/hbm.24753 


\subsection{Abstract}

This study investigated volumetric brain changes and cognitive performance in pre- and post-menopausal patients treated for early-stage breast cancer. Participants underwent elaborate neurocognitive assessments (neuropsychological testing, cognitive failure questionnaire and high-resolution T1-weighted structural MRI) before and after chemotherapy. Volumetric brain changes were estimated, using longitudinal deformation-based morphometry, and correlated with cognitive changes.

In total, 180 women participated in this study, of whom 72 patients with breast cancer had received adjuvant chemotherapy $(\mathrm{C}+), 49$ patients did not receive chemotherapy $\left(\mathrm{C}_{-}\right)$and 59 healthy controls $(\mathrm{HC})$. The population was categorized into two age groups: a young group who were pre-menopausal and younger than 52 years at baseline $(n=55 \mathrm{C}+/ 32 \mathrm{C}-/ 41 \mathrm{HC})$, and an older group who were post-menopausal and older than 60 years $(n=17 \mathrm{C}+/ 17 \mathrm{C}-/ 18 \mathrm{HC})$.

Cognitive impairment occurred after chemotherapy in both young and older patients, although older patients showed more decline in processing speed (Trailmaking test $b$ ). White matter volume expansion was observed after chemotherapy, only significantly present in the younger subgroup of patients. In patients not treated with chemotherapy, diffuse grey and white matter volume reduction was observed. Less white matter expansion concurred with more cognitive decline $(r>.349, p<.05)$.

In conclusion, we found age-dependent cognitive decline and white matter volume changes in patients with breast cancer after chemotherapy, which could possibly be linked to neuroinflammatory processes. White matter expansion after chemotherapy, more pronounced in pre-menopausal patients, correlated with less cognitive decline. This suggests such expansion to be age-dependent, possibly caused by a protective response in the younger brain to chemotherapy-induced neurotoxicity. 


\subsection{Introduction}

Eight percent of women in developed countries are confronted with breast cancer during their lifetime ${ }^{135}$. The last 25 years, improvements in systemic treatment and screening have drastically improved survival rates ${ }^{136}$. However, up to $70 \%$ of chemotherapy-treated patients experience cognitive problems, frequently affecting memory, executive function, processing speed and attention. This can have a lasting impact on the patient's quality of life $e^{21 ; 38}$.

The pathophysiology of chemotherapy-induced cognitive impairment remains largely unknown $^{21 ; 37 ; 38}$. Multiple hypotheses of (in)direct pathways have been proposed, including neuro-inflammation, neurotoxicity, oxidative stress, hormonal dysregulation and genetic predisposition $^{21 ; 37 ; 137 .}$.

Despite age being a well-known risk factor for the incidence of cancer ${ }^{138}$ and neurodegenerative processes ${ }^{139}$, few studies have explored interactions of age and cognitive functioning after cancer treatment ${ }^{140 ; 141}$. Ahles et al. ${ }^{140}$ found older patients to perform worse on processing speed measurements. Similarly, Schilder et al. ${ }^{141}$ found older patients treated with endocrine treatment scored lower on more cognitive domains than younger patients. Both studies suggest age to amplify the cognitive effects of chemotherapy ${ }^{140}$ and hormonal therapy ${ }^{141}$. Hypothesized underlying mechanisms include a weakened blood-brain-barrier, stronger inflammatory reactions, coinciding neurodegeneration, delayed neural repair and hormonal changes ${ }^{33}$. Assessing cognitive impact of cancer treatment in elderly, especially when frailty (i.e. aging-associated increased vulnerability due to loss of physiological reserve ${ }^{142}$ ) co-occurs, is of high importance as therapy could accelerate cognitive aging and increase the risk of dementia ${ }^{143}$.

In an attempt to unravel the underlying neural correlates, multiple studies have investigated morphometric brain changes after chemotherapy using voxel-based morphometry (VBM) ${ }^{22}$. These studies reported lower total brain volume and widespread lower grey matter (GM) density/volume compared to controls ${ }^{22 ; 35}$. Longitudinal VBM analyses by Lepage et al. ${ }^{144}$ and McDonald et al. ${ }^{24 ; 32}$ found widespread GM density/volume decrease one month after chemotherapy compared to baseline, with partial recovery one year later $24 ; 32 ; 144$. Only a few studies reported white matter (WM) volumetric changes ${ }^{22 ; 145}$. However, VBM is known to have a low sensitivity in the latter tissue ${ }^{146}$.

In contrast to VBM, deformation-based morphometry (DBM) does not rely on tissue segmentation, but on the deformation field derived from image registration, offering a direct method for investigating global volumetric brain changes ${ }^{115 ; 147 ; 148}$. Hence, DBM could provide new insights in the neural substrate of aging and chemotherapy-induced cognitive changes. 
Therefore, we used DBM to assess brain volume changes after cancer treatment and its relationship to cognitive performance and age within the context of a longitudinal study, in patients with early-stage breast cancer. Based on the aforementioned studies, we hypothesized widespread brain volume changes and cognitive decline after chemotherapy, more pronounced in older patients.

\subsection{Methods}

\subsubsection{Participants}

A total of 180 women participated in this study, of whom 72 patients with early-stage breast cancer had received adjuvant chemotherapy $(\mathrm{C}+), 49$ patients did not receive chemotherapy $\left(\mathrm{C}_{-}\right)$ and 59 healthy controls (HC). The population was recruited into two separate age groups: a young group who were pre-menopausal and younger than 52 years at baseline $(n=55 C+$, $32 \mathrm{C}$ - and $41 \mathrm{HC}$ ), and an older group who were post-menopausal and older than 60 years at baseline $(\mathrm{n}=17 \mathrm{C}+, 17 \mathrm{C}$ - and $18 \mathrm{HC})$. Participants were excluded in case of history of cancer or a neurological/psychiatric condition. All participants were tested and recruited at the university hospitals of Leuven between 2008 and 2014.

Surgery was performed in both $\mathrm{C}+$ and $\mathrm{C}$ - patients. Thereafter, $\mathrm{C}+$ were treated with one of two standardized chemotherapy protocols, consisting of either six cycles of FEC $\left(500 \mathrm{mg} / \mathrm{m}^{2}\right.$ Fluorouracil, $100 \mathrm{mg} / \mathrm{m}^{2}$ Epirubicin and $500 \mathrm{mg} / \mathrm{m}^{2}$ Cyclophosphamide) or three cycles of FEC and three cycles of Docetaxel $\left(100 \mathrm{mg} / \mathrm{m}^{2}\right)$. Chemotherapy cycles were standard administered at three-week intervals, except when contra-indications were present. After the end of chemotherapy in the $\mathrm{C}+$ group or surgery in the $\mathrm{C}$ - group, some patients additionally received radiotherapy and/or hormonal therapy.

Baseline assessment ( $\mathrm{t} 1$ ) was performed after surgery, before chemo- or radiotherapy was initiated. Post-treatment assessment ( $\mathrm{t} 2$ ) was conducted after completion of both chemo- and radiotherapy (when applicable). Hormonal therapy was already started for some patients at $\mathrm{t} 2$. This corresponded to a mean time interval of 5-6 months after end of chemotherapy. HC and Cparticipants were assessed at matched intervals. The study was approved by the local ethical commission and conducted in accordance with the Declaration of Helsinki. The younger group in this study consists of the populations described in Deprez et al. ${ }^{23 ; 28}$ (Table 4.1), supplemented with $4 \mathrm{C}+$ patients. However, these publications analyzed cognition and functional/diffusion MRI data. 
All data that support the findings of this study are available from the corresponding author upon reasonable request.

\subsubsection{Neuropsychological assessment}

All participants completed the Cognitive Failure Questionnaire (CFQ) ${ }^{149}$ to evaluate self-reported cognitive functioning, providing five subscales. Anxiety was assessed with the Spielberger State-Trait Anxiety Inventory (STAI) ${ }^{150}$, depressive symptoms with the Beck Depression Inventory (BDI) ${ }^{151}$ and verbal intelligence-quotient with the Dutch Adult Reading Test ${ }^{152}$. More extensive neuropsychological assessments were acquired in a subset of participants (12 tests, 31 outcome measurements), as described previously ${ }^{28}$ (Table 4.1).

\subsubsection{Magnetic Resonance Imaging}

Three-dimensional T1-weighted images were acquired on a 3 Tesla scanner (Intera; Philips Medical Systems, Best, the Netherlands) with an 8-channel head coil (TR/TE/TI 9.6/4.6/900ms, FOV $250 \times 250 \times 218 \mathrm{~mm}$, voxel size $0.98 \times 0.98 \times 1.20 \mathrm{~mm}$ ). All scans were acquired on the same scanner with unchanged configuration.

Images were processed using ANTs N4-biasfield correction ${ }^{153}$, followed by the longitudinal processing pipeline of the CAT12 ${ }^{154}$ SPM12 toolbox. Jacobian maps were calculated from the deformation matrices using symmetric longitudinal registration ${ }^{155}$, providing a direct measure of brain volume changes. These maps were smoothed with a Gaussian filter with $8 \mathrm{~mm}$ isotropic FWHM. Visual inspection was performed on both raw and registered data to ensure data quality. Images with extensive motion artifacts were excluded.

Regional volume changes were calculated for the correlation analyses with neuropsychological outcome. As volume changes after chemotherapy were most prominent within the WM, the John Hopkins University WM atlas ${ }^{156}$ was applied to calculate the relative volume change within different WM regions.

\subsubsection{Statistical analysis}

Statistics of neuropsychological testing and correlation analysis were performed with SPSS v25. At baseline, one-way ANOVA was used to assess group differences and two-way ANOVA to assess age and group interaction effects. Ordinal clinical data was compared between young and old subgroups using Mann-Whitney U-test. Fisher's exact test was used to compare frequencies. 
Paired t-tests were used to assess changes ( $\mathrm{t} 1$ vs. $\mathrm{t} 2$ ) in neuropsychological performance within each subgroup. We used one-way RM-ANOVA to assess time-by-group interactions. Similarly, time-by-age effects were investigated for each group separately. In all analyses, BDI and IQ score were included as covariates ${ }^{157}$. Statistical significance was assessed at $p<.05$ with Hochberg correction for multiple outcomes ${ }^{158}$.

We performed a GLM analysis of the Jacobian maps using SPM $12^{159}$, including the interactions between age (young vs. old), time ( $\mathrm{t} 1$ vs. $\mathrm{t} 2$ ) and group $(\mathrm{C}+, \mathrm{C}$ - and $\mathrm{HC})$. A pairing factor was included for each subject. BDI and scanner hardware maintenance (less than 2 months before maintenance of a RF transmitter coil or RF amplifier) were included as covariates ${ }^{160-162}$. All reported $\mathrm{p}$-values were family-wise-error corrected on cluster level at $p<.05$, with an uncorrected voxel-level threshold of $p<.001$. Two independent neuroradiologists (AR and SS) interpreted the resulting statistical maps.

Explorative Pearson correlations were used to examine the relationship between changes in self-reported measures or neuropsychological performance and brain volume changes for young and older $\mathrm{C}+$ patients separately. To reduce the number of tests, we only included neuropsychological tests showing a significant time-by-group interaction, total CFQ score. Five regions were selected by overlaying the JHU-atlas with the parametric maps of significant volume changes in both age subgroups and age-by-time interactions.

\subsection{Results}

\subsubsection{Demographic and medical data}

An overview of all participants is included in Table 4.1. Two young $C+$ patients were excluded due to a disproportionate time interval ( $>5 S D$ above mean) between the last administered chemotherapy and t2 (367 and 392 days, respectively). One young HC was excluded as outlier in time between $\mathrm{t} 1$ and $\mathrm{t} 2$ of 812 days ( $>6 S D$ above mean). Six participants had incomplete imaging data $(2 \mathrm{C}+, 3 \mathrm{C}-, 1 \mathrm{HC})$. Because of extensive motion artefacts, six participants were excluded from imaging analysis $(2 \mathrm{C}-, 4 \mathrm{HC})$. Cognitive failure questionnaires were missing for seven participants $(3 \mathrm{C}+, 1 \mathrm{C}-, 3 \mathrm{HC})$. Neuropsychological assessment was missing for two young C+ participants.

Table 4.2 summarizes demographics and medical information. Disease stage was similar for young compared to old patients in both cancer groups. However, $\mathrm{C}+$ patients were more often diagnosed with stage II to III cancer, compared to C- patients. BDI scores were higher in 
Table 4.1 - Overview of the total population size and subdivision into samples. (1): $34 \mathrm{C}+, 16 \mathrm{C}-$ and 19 HC described in Deprez et al., $2012^{28}$ corresponding to $92 \%$ of the population in sample 1. (2): population described in Deprez et al., 2014 ${ }^{23}$. Initial sample size and start and end dates of data acquisition are described, as well as sample size remaining after exclusion of outlier, missing and artefact-ridden data. NP: neuropsychological assessment; $\mathrm{C}+$ : patients receiving chemotherapy; C-: patients not receiving chemotherapy; HC: healthy controls; CFQ: cognitive failure questionnaire. *Exclusions due to: missing data, motion artifacts (MRI) or disproportionate time interval between therapy and testing $(>5 S D)$ or between consecutive testing $(>6 S D)$.

\begin{tabular}{|c|c|c|c|c|c|c|}
\hline & \multicolumn{4}{|c|}{$\begin{array}{c}\text { Young group } \\
56 \mathrm{C}+, 33 \mathrm{C}-\text { and } 37 \mathrm{HC}\end{array}$} & \multicolumn{2}{|c|}{$\begin{array}{c}\text { Old group } \\
17 \mathrm{C}+, 17 \mathrm{C}-\text { and } 18 \mathrm{HC} \\
\end{array}$} \\
\hline & & Sample $1^{(1)}$ & & Sample $2^{(2)}$ & & Sample 3 \\
\hline Start date & & $17 / 01 / 2008$ & & $28 / 12 / 2009$ & & $29 / 07 / 2008$ \\
\hline End date & & $22 / 10 / 2012$ & & $27 / 09 / 2012$ & & $08 / 05 / 2014$ \\
\hline Group size & & $39 \mathrm{C}+, 16 \mathrm{C}-, 20 \mathrm{HC}$ & & $17 \mathrm{C}+, 17 \mathrm{C}-, 17 \mathrm{HC}$ & & $17 \mathrm{C}+, 17 \mathrm{C}-, 18 \mathrm{HC}$ \\
\hline Available data & MRI & $37 \mathrm{C}+, 15 \mathrm{C}-, 19 \mathrm{HC}$ & MRI & $16 \mathrm{C}+, 16 \mathrm{C}-, 14 \mathrm{HC}$ & MRI & $17 \mathrm{C}+, 14 \mathrm{C}-, 16 \mathrm{HC}$ \\
\hline (after exclusion*) & CFQ & $35 \mathrm{C}+, 16 \mathrm{C}-, 17 \mathrm{HC}$ & CFQ & $17 \mathrm{C}+, 17 \mathrm{C}_{-}, 17 \mathrm{HC}$ & CFQ & $17 \mathrm{C}+, 16 \mathrm{C}-, 17 \mathrm{HC}$ \\
\hline & NP & $36 \mathrm{C}+, 16 \mathrm{C}-, 19 \mathrm{HC}$ & & & & $17 \mathrm{C}+, 17 \mathrm{C}-, 18 \mathrm{HC}$ \\
\hline
\end{tabular}

treatment groups than controls but were consistent between the age groups. STAI scores were significantly higher in the old $\mathrm{C}+$ patients than in the young subgroup, with similar trends for $\mathrm{C}$ patients. Most patients received additional radiotherapy (>70\%) and hormonal therapy $(65 \%)$, with radiotherapy being significantly more administered in old than young $\mathrm{C}$ - patients. Years of education was slightly higher in both young treatment groups compared to the old subgroups, though verbal IQ was similar between age groups. In HC, IQ was slightly higher (5 points on average) in the older subgroup. Slight test-retest time variations exist $(<24$ days on average between age subgroups), which were significant for $\mathrm{C}+$ and $\mathrm{HC}$.

\subsubsection{Neuropsychological assessment}

Neuropsychological results are described in Tables 4.3, 4.4 and supplementary Table 8.1.

At baseline, no differences in neuropsychological performance or subjective measures between patients and controls were observed, after correction for BDI and IQ. Group-by-time interactions revealed $C+$ patients $(n=53)$ performed significantly worse after chemotherapy on attention/concentration, memory and processing speed tests, while both control groups $(n=37 \mathrm{HC}$, 33C-) showed increased performance on the same tests (Table 4.3).

Main effects of age were found on all neuropsychological test domains at baseline, with older participants $(n=52)$ performing consistently worse than younger participants $(n=71)$ (Table 4.4). No group-by-age interactions were found at baseline. When evaluating effects of age on cognitive decline, time-by-age interactions revealed older chemotherapy-treated patients $(n=17)$ scored lower for processing speed (Trailmaking test $b)$, while younger patients $(n=36)$ improved on this domain. A similar trend was seen for executive functioning (Stroop). A 


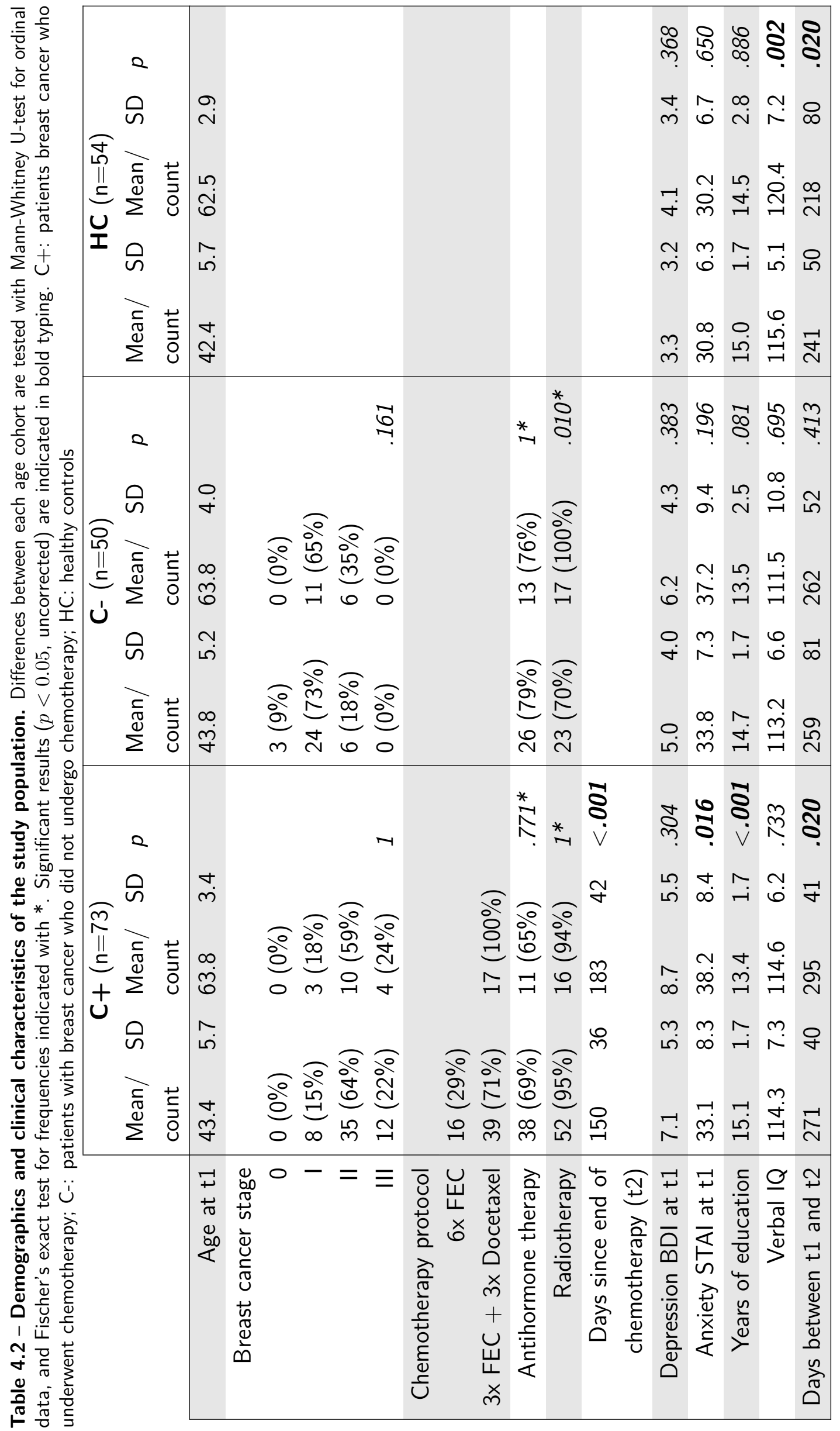




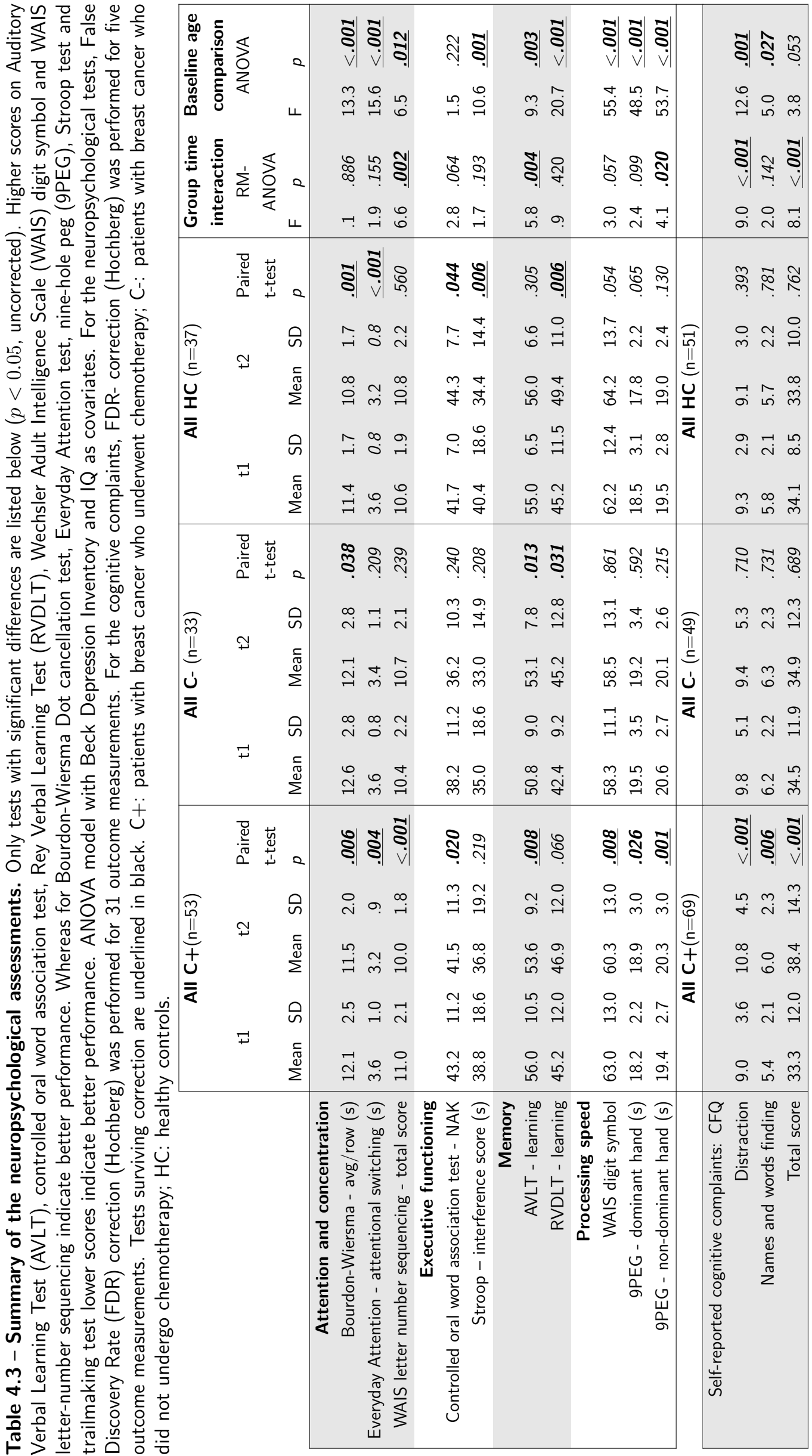


time-by-age interaction for the Everyday Attention test showed older patients to improve throughout treatment, approaching the average score of younger patients, whom did not improve over time. No time-by-age interactions were found in $\mathrm{C}$ - and $\mathrm{HC}$.

At baseline, a main effect of age was found for the self-reported subscale of distraction in social situations and names and word finding; older participants reported being more distracted (Table 4.4). No group-by-age interaction at baseline, nor time-by-age interaction for the treatment groups separately, was found. However, paired t-tests revealed younger participants reported more cognitive complaints after chemotherapy compared to baseline, whereas this was not significant for older patients. 


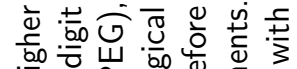
主命高

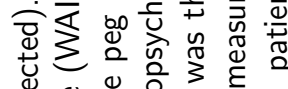

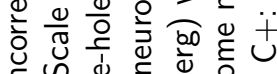

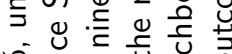
28.

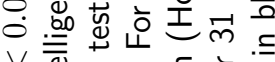

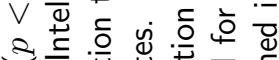

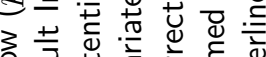

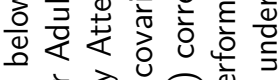

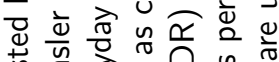
类点

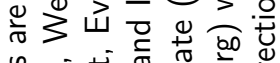

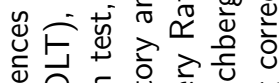

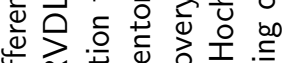

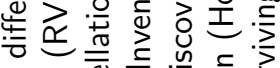

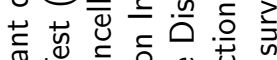

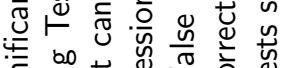
告

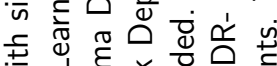

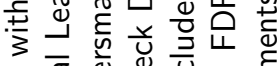

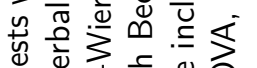

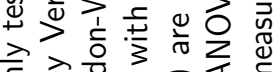

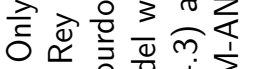
ज்

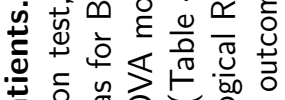

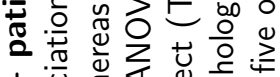

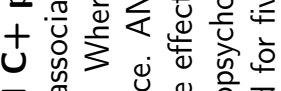
등 宁

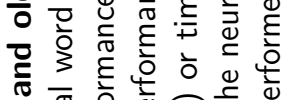
ब。

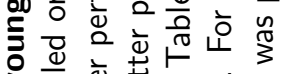

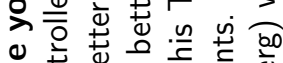

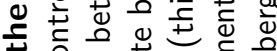

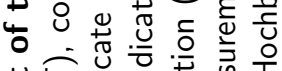

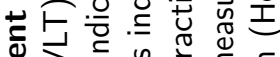

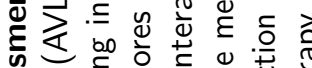
प्र

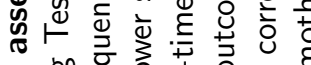
万ٓ

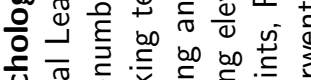

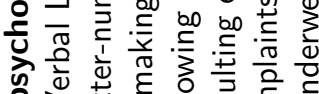

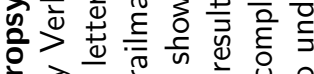

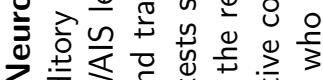

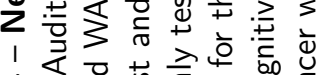

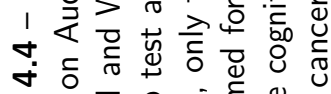

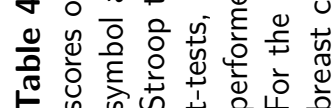

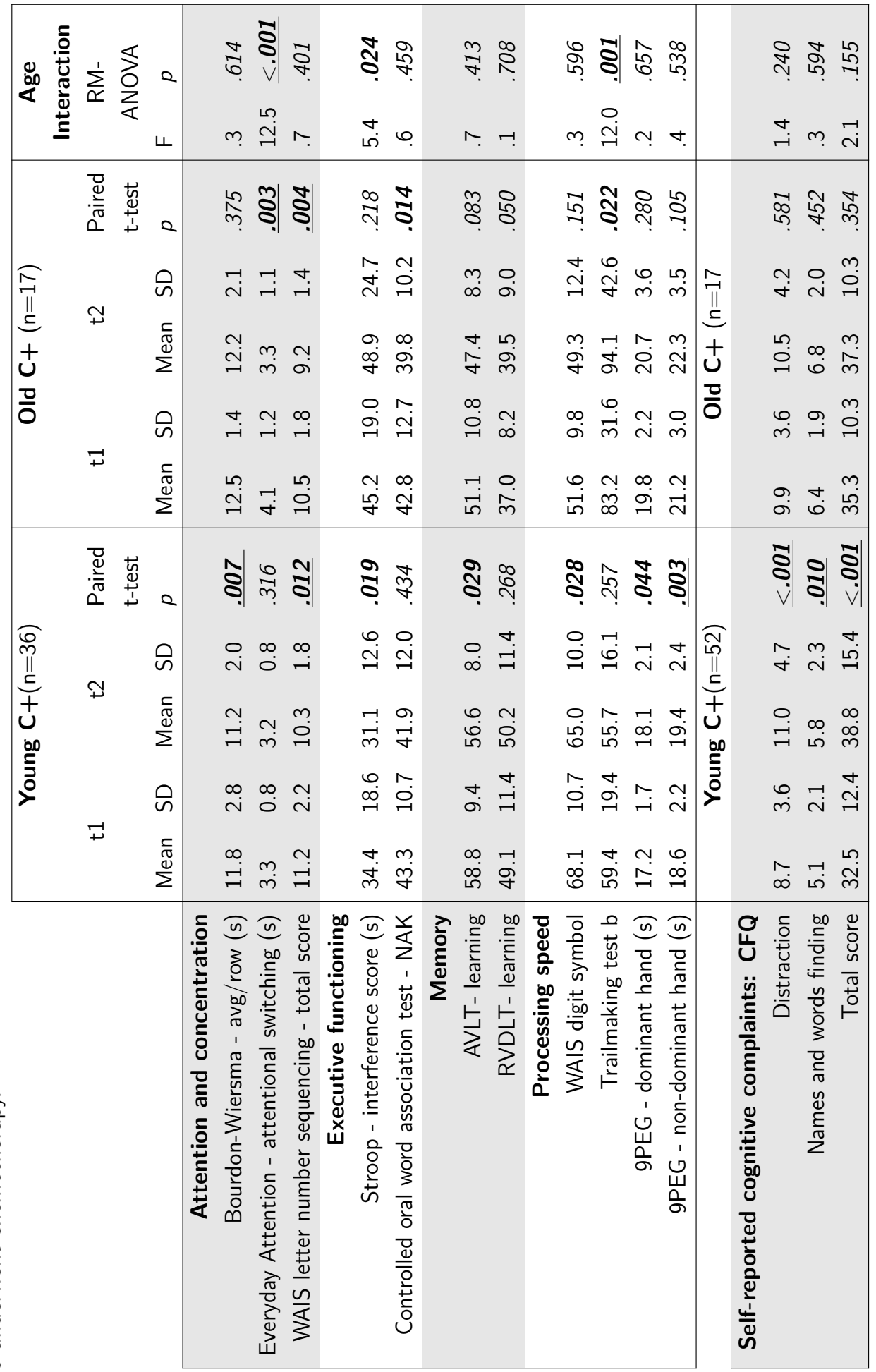




\subsubsection{Deformation-based morphometry}

DBM analysis showed widespread WM volume expansion 5-6 months ( $\mathrm{t} 2 \mathrm{vs.} \mathrm{t} 1$ ) after end of chemotherapy in $\mathrm{C}+$ patients, accompanied with volume reductions in the ventricles and interhemispheric fissure (Figure 4.1:A). In C-patients, volume decrease was found throughout multiple GM regions, extending into WM. Associated volume increase was found in the dorsal interhemispheric fissure (Figure 4.1:B). No significant changes were detected in HC (Figure 4.1:C).

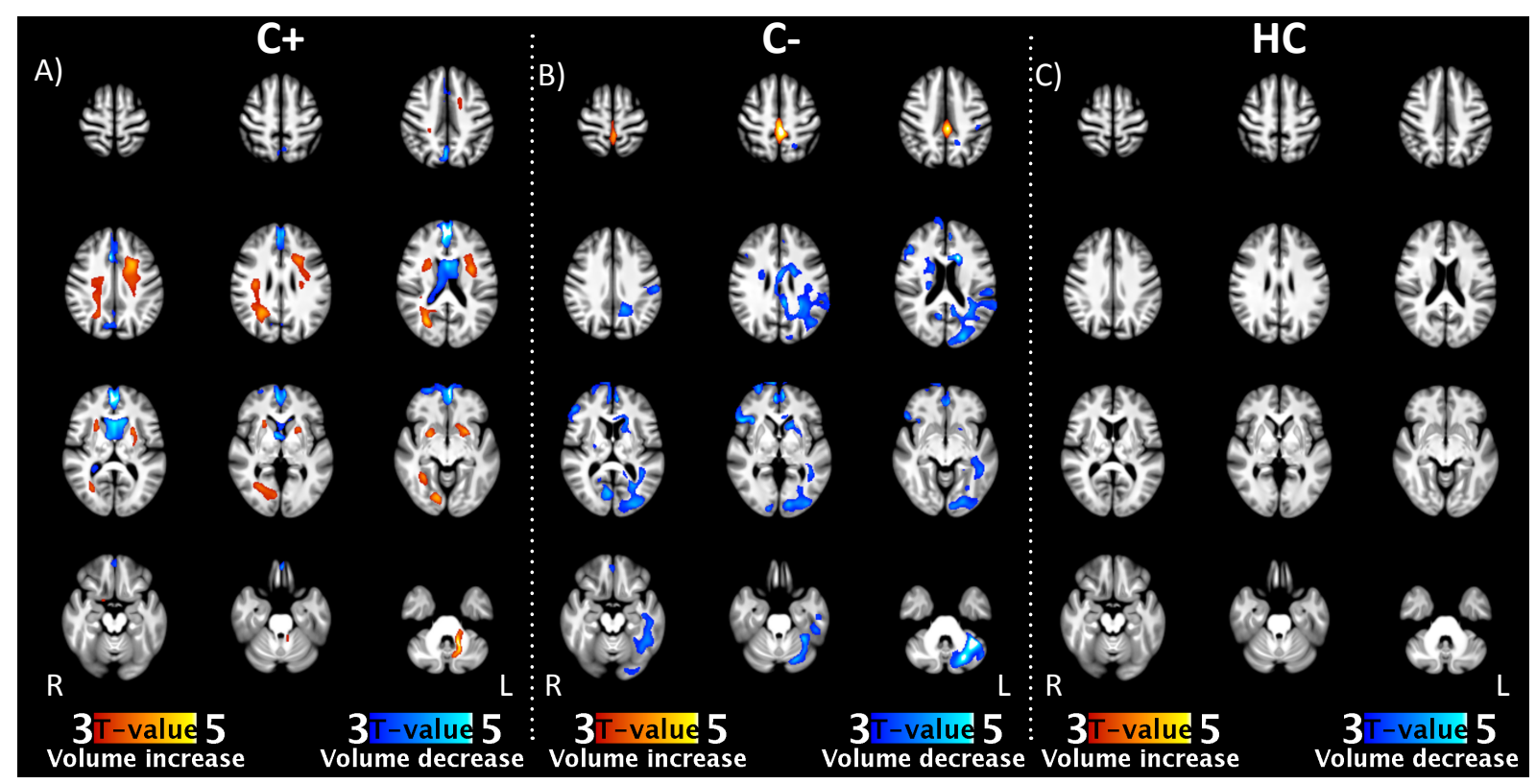

Figure 4.1 - Within-group volumetric changes of all groups, independent of age, comparing time point $\mathbf{t} 2$ with baseline. Brain volume changes were found in both cancer groups $(C+: n=70, C-: n=45)$. No effects were found in the healthy control group ( $\mathrm{HC}: \mathrm{n}=49)$. Statistical maps of volume increase and decrease are thresholded at $p<0.05$, FWE corrected at cluster level, and color coded according to T-value. See supplementary Table 8.2 on statistical values and MNI-coordinates of significant clusters. C+: patients breast cancer who underwent chemotherapy; C-: patients with breast cancer who did not undergo chemotherapy; HC: healthy controls.

With regard to age effects, widespread WM expansion was observed in the younger $\mathrm{C}+$ group, alongside volume reductions in the CSF of the ventricles, interhemispheric fissure and multiple cortical sulci, extending into the GM of the medial prefrontal cortex (MPFC) (Figure 4.2:A). By contrast, no significant changes were found in older $\mathrm{C}+$ patients (Figure 4.2:B). Age-by-time interactions in the $\mathrm{C}+$ group confirmed more WM expansion in the young compared to the older group, in right frontal and temporal regions, combined with volume reductions in the ventricles and the frontal interhemispheric fissure (Figure 4.2:C). In C- patients, both young and old women (Figure 4.2:D,E) showed volume decreases. In young patients this was observed in the MPFC, fusiform gyrus and left cerebellar hemisphere. In older patients these patterns appeared in the bilateral cingulate cortices, as well as left frontal, parietal, occipital regions, basal ganglia and anterior corpus callosum. For the younger subgroup, volume increase was found in the dorsal 
interhemispheric fissure. The age-by-time interaction indicated more volume decrease in the older subgroup in the subcortical WM and GM of the left orbitofrontal and middle frontal gyri (Figure 4.2:F). 


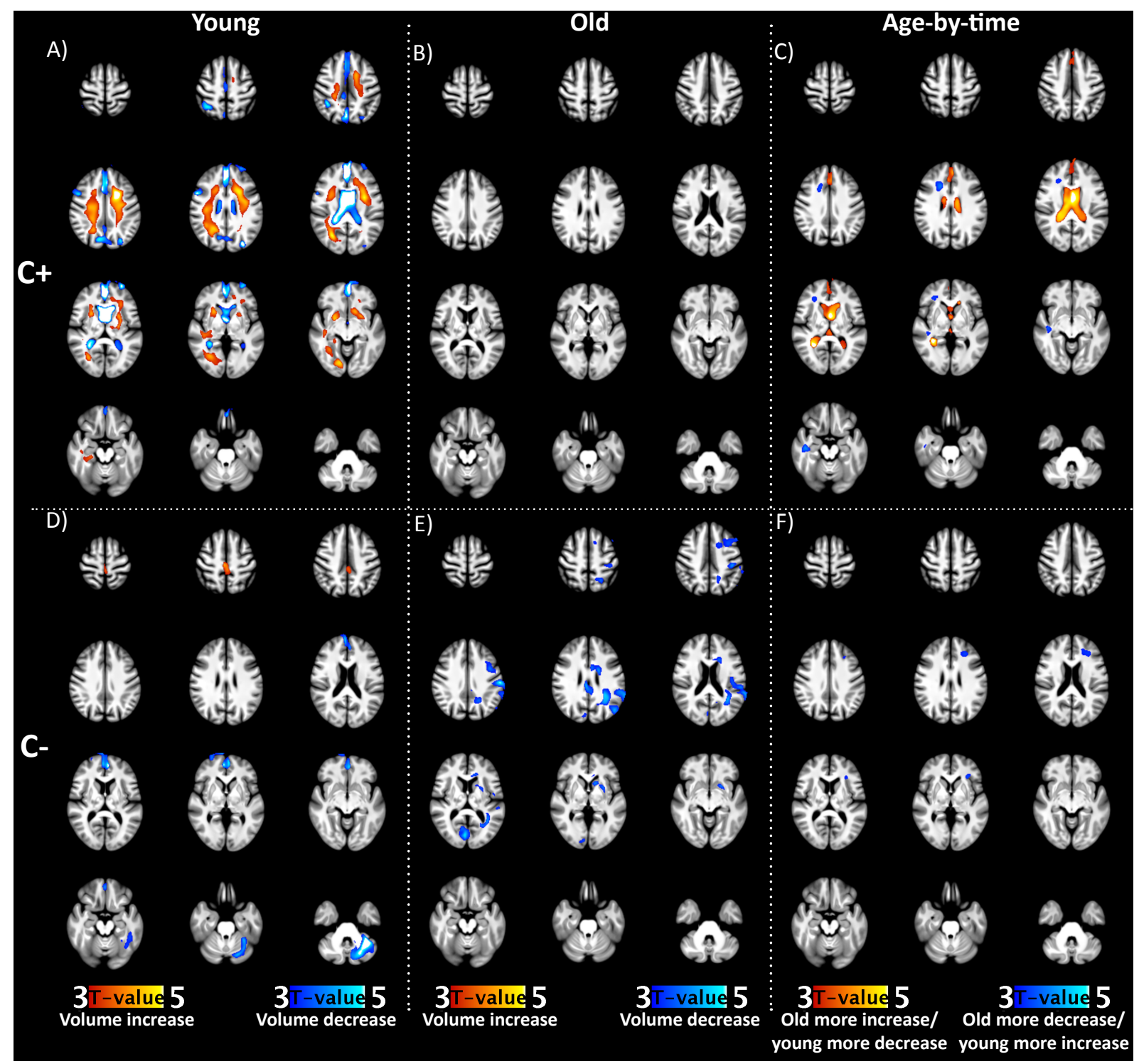

Figure 4.2 - Comparison of the volumetric changes in young and old women after cancer therapy. Changes are shown for $C+($ row $1, A-C)$ and $C-($ row 2, D-F) patients. The first $(A, D$ with $n=53,3$ ) and second $(B, E$ with $n=17,14)$ column show regions of significant volume increase and decrease over time for the different young (pre-menopausal) and old (post-menopausal) groups, respectively. The last column (C, F) show regions with a significant time-by-age interaction effect for each group. All statistical maps are thresholded at $p<0.05$, FWE corrected at cluster level, and color coded according to T-value. See supplementary Tablee 8.3 for statistical values and $\mathrm{MNI}$-coordinates of significant clusters. $\mathrm{C}+$ : patients breast cancer who underwent chemotherapy; $\mathrm{C}_{-}$: patients with breast cancer who did not undergo chemotherapy. 


\subsubsection{Correlation analysis of neuropsychological testing and WM vol- ume changes}

Explorative correlation analyses were performed for $\mathrm{C}+$ patients between change in volume and performance on neuropsychological tests and cognitive complaints. Since only $\mathrm{C}+$ patients showed cognitive decline, correlations were only performed for this group. Regions included: right/left corona radiata, right/left superior longitudinal fasciculus (SLF) and the right sagittal stratum. In the older subgroup, a correlation was found between decreased verbal memory $(\mathrm{AVLT})$ and more volume reduction in the right corona radiata $(r=.484, p=.049$, Figure 4.3:B). For young $\mathrm{C}+$ patients, correlations were found between decreased attention/concentration (WAIS letter-number sequencing) and less volume increase in the right corona radiata $(r=.349$, $p=.040$, Figure 4.3:A) and left SLF $(r=.354, p=.037$, Figure 4.3:C). Cognitive complaints did not correlate with regional WM volume changes in either age groups. 
A

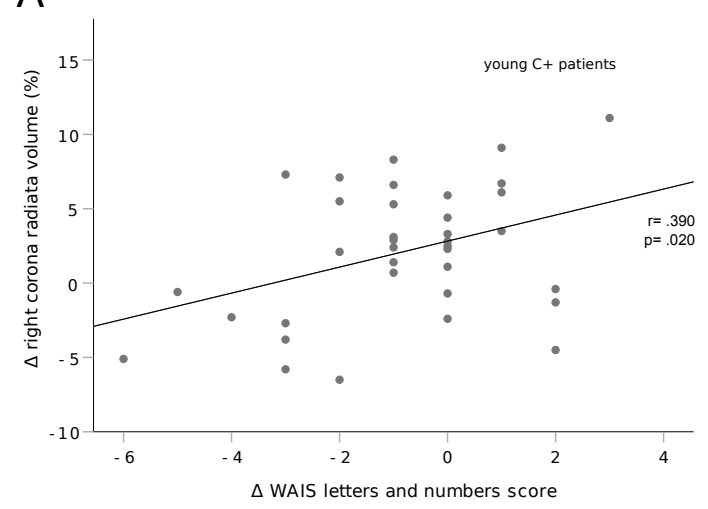

C

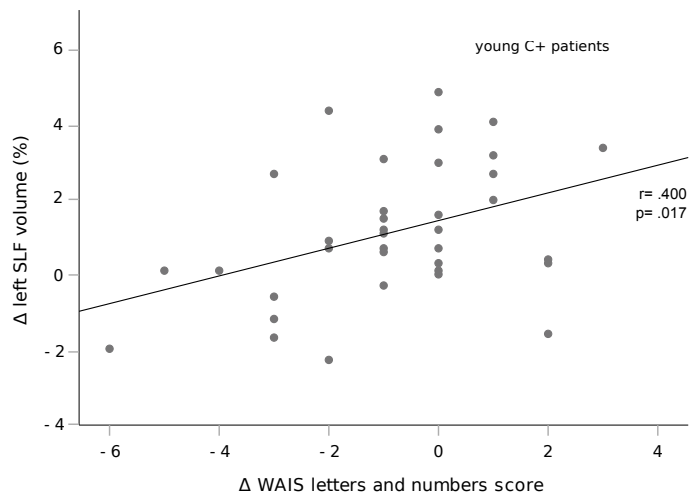

B

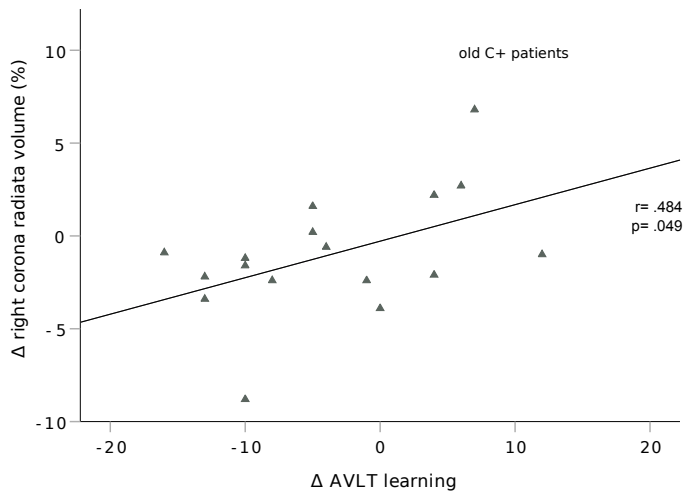

Figure 4.3 - Correlation of changes in cognitive scores with changes in brain volume of young and old C+ patients. (A, B) Scatter plots of percental longitudinal change in left corona radiate brain volume and WAIS letter-number sequencing change score in young patients (A) and AVLT learning in old patients (B). (C) Scatter plot of percental longitudinal change in left SLF volume and WAIS letter-number sequencing change score in young patients. Change scores were calculated as $\mathrm{t} 2 \mathrm{score}-\mathrm{t} 1 \mathrm{score}$. For the percental volume change these scores were divided by the baseline volume. A higher change score on the WAIS letter-number sequencing and AVLT test indicate better performance. Positive brain volume change indicates volume expansion, while negative values indicate decrease in brain volume. AVLT: Auditory Verbal Learning Test, C+: patients breast cancer who underwent chemotherapy; SLF: superior longitudinal fasciculus; WAIS: Wechsler Adult Intelligence Scale. 


\subsection{Discussion}

This longitudinal study investigated cognitive functioning and brain volume changes after chemotherapy for breast cancer, and its interaction with aging. Cognitive impairment occurred after chemotherapy in both young and older patients, although older patients experienced more processing speed decline. Widespread WM volume expansion was observed 5-6 months after chemotherapy, only significantly present in young patients. In patients with cancer not treated with chemotherapy, diffuse GM/WM volume decreases were observed, with more volume decrease in the left frontal lobe of the older subgroup. Less WM expansion after chemotherapy correlated with cognitive decline.

The findings of decreased attention/concentration, memory and processing speed after chemotherapy are in concordance with previous longitudinal studies ${ }^{33 ; 163}$. Additionally, this study found older patients receiving chemotherapy to be more vulnerable with regard to processing speed. This corroborates the study of Ahles et al. ${ }^{140}$, investigating interactions between age and chemotherapy, which found more processing speed decline in older patients with less cognitive reserve. However, the majority of included patients received additional endocrine treatment, which could also have a cognitive impact ${ }^{141}$. Improvement on the Everyday Attention test only in older patients could be explained by a ceiling effect, since younger patients reached the same post-therapy average score as healthy volunteers ${ }^{164}$. Nevertheless, younger patients reported more subjective cognitive decline. This suggests daily-life experienced cognition depends on age-modulated social expectations, which can be difficult to capture with objective tests. While no interactions between age and cognitive complaints have been found in an earlier study using a different questionnaire ${ }^{140}$, this finding adds to current recognition of young, highly educated women reporting persistent cognitive changes after chemotherapy ${ }^{165}$.

To the best of our knowledge, this study is the first to observe WM expansion shortly after adjuvant chemotherapy in patients. However, a recent study by Winocur et al ${ }^{166}$, using a mouse-model of breast cancer, showed chemotherapy to cause frontal brain volume enlargement. Moreover, earlier diffusion tensor imaging studies in this population reported widespread increased mean diffusivity and decreased fractional anisotropy in $W^{28 ; 167}$. A potential mechanism underlying such microstructural and volumetric changes could be edema formation, as a chemotherapy-induced neuro-inflammatory mechanism ${ }^{37}$. However, further research is necessary to confirm this hypothesis.

The chemotherapeutic agents used in our sample are known to have (in)direct neurotoxic effects. 5-fluorouracil for example, is known to cross the blood-brain barrier by simple diffusion ${ }^{39}$ and causes WM changes in mice ${ }^{137}$. Besides direct toxic mechanisms, indirect processes such as 
inflammatory responses can also affect neural integrity, which was evidenced in rodents for multiple chemotherapeutic agents administered in our population ${ }^{42}$.

The lack of significant WM volume increase in old $\mathrm{C}+$ patients suggests an interaction between neural changes induced by aging, hormonal changes and chemotherapy. There are multiple aging candidate mechanisms for modulating this WM expansion: a primed pro-inflammatory state in the brain ${ }^{168-170}$, delayed recruitment of microglia ${ }^{171}$ and phagocytic cells ${ }^{172}$ to the injured site, age-related neurodegeneration ${ }^{170}$, changes in brain viscoelasticity ${ }^{173}$, blood-brain-barrier changes $^{33}$, CSF changes slowing down excretion of neurotoxic metabolites ${ }^{174}$ and poorer tissue regeneration ${ }^{170}$. This multitude of interacting processes could result in inefficient and/or delayed responses to neurotoxic effects of chemotherapy. Additionally, the different menopausal status in both age groups could have played a role. For example, estrogen is known to have a neuroprotective function ${ }^{33 ; 175}$. Moreover, given the well-known pro-inflammatory state present in the aged brain ${ }^{168-170}$, it is unlikely the reduced volumetric response in the older $\mathrm{C}+$ subgroup was caused by decreased neuro-inflammation. Rather, the different volumetric brain patterns are presumably caused by a different progression of neuro-inflammation with age, combined with an altered response to neuro-inflammation in the aged brain ${ }^{173}$.

In C+ patients, less pronounced WM swelling in either SLF or corona radiata correlated with more cognitive decline after chemotherapy; for attention and processing-speed domains in young patients and memory in old patients. This result adds to the hypothesis of the observed WM enlargement as a 'protective' response to neurotoxicity, which is more pronounced in young patients. Both WM regions found in this correlation analysis are known to be involved in higher cognitive functioning. The SLF, a long association tract between frontal and parietal cortices, is known to be linked with processing speed in healthy volunteers ${ }^{176}$ and attention in MS patients ${ }^{177}$. Likewise, the corona radiata has been linked to attention, executive functioning and memory domains ${ }^{178}$. Previous studies from our group on a subset of this data revealed correlations between microstructural WM changes of the corona radiata and SLF with attention and verbal memory tests ${ }^{28}$.

The GM volume decrease found in the C-group could reflect neural changes after cancer therapy, apart from chemotherapy. These changes can be attributed to a combination of other cancer therapies (radiotherapy ${ }^{20}$, hormonal therapy ${ }^{179 ; 180}$ ), cancer itself ${ }^{166}$, and interactions with anxiety ${ }^{181}$, stress ${ }^{181}$, and fatigue ${ }^{182}$. Specifically, a rodent study found tumorigenic mice to have decreased regional brain volume, while control mice treated with chemotherapy (5-Fluorouracil and methotrexate) showed increased volume ${ }^{166}$. This preclinical observation suggests chemotherapy-induced effects to potentially mask cancer-induced GM decrease. Moreover, as DBM, compared to VBM, is less sensitive to mesoscopic volume changes ${ }^{115}$, 
previously observed GM decrease ${ }^{24 ; 32 ; 144}$ might be missed. This might partially explain the discrepancy between our findings and previous studies.

Some limitations of this study need to be mentioned. First, the sample size of the older subgroup remained limited, especially when compared to the younger subgroup. Still, the observed interaction effects between age and patient groups took the different sample sizes into account and confirmed the observed within-group differences. Second, breast cancer stage was significantly different between the patients treated with $(\mathrm{C}+)$ and without $\left(\mathrm{C}_{-}\right)$chemotherapy in both age groups. Although our results point to chemotherapy-induced cognitive changes, the observed responses could still be a combinational effect of cancer-induced changes, hormonal therapy and chemotherapy. More specifically, as depicted in Table 4.2, the majority of C+ and Calso receive hormonal treatment. Although, at $\mathrm{t} 2$, hormonal treatment was only received for a short period of time, this might have modulated the observed effects. Moreover, by design of the study, both age groups differed in age as well as menopausal status. By consequence, further research is needed to distinguish the impact of both underlying factors. Next, slight test-retest variations were observed between groups. However, when we added this variation as a covariate in the imaging analysis, our results remained consistent. Lastly, future studies should investigate metabolic and physiological changes after chemotherapy more directly and during a longer follow-up, to confirm the hypothesis of neuro-inflammation induced WM expansion, modulated by aging and hormonal changes.

To the best of our knowledge, this study is the first reporting an age-modulated volumetric brain response to chemotherapy and its relation to cognition. We observed widespread WM enlargement after chemotherapy, only significantly present in the younger brain, suggested as a response to chemotherapy-induced neuro-inflammation. Patients with breast cancer who did not receive chemotherapy showed GM volume reduction, indicating a potential impact of the cancer itself and/or the therapies apart from chemotherapy on neurodegeneration. Older chemotherapy patients showed to be more vulnerable to cognitive decline than their younger counterparts, while showing no WM enlargement after chemotherapy. Less WM expansion correlated with more cognitive decline, suggesting this volume response to originate from a protective mechanism against the neural impact of chemotherapy. 


\section{CHAPTER}

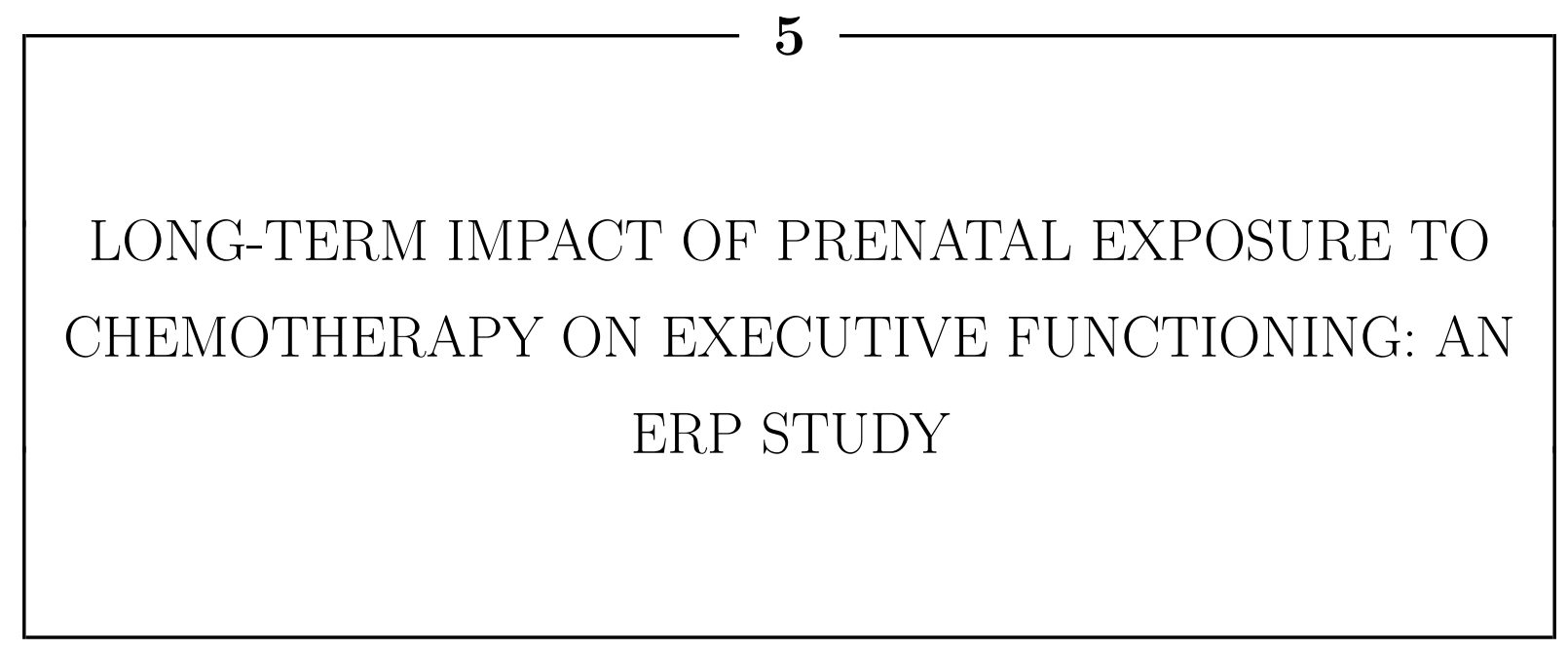

This chapter has been published as:

Blommaert J., Zink R., Deprez S., Myatchin I., Dupont P., Vandenbroucke T., Sleurs C., Van Calsteren K., Amant F. and Lagae L. (2019). Long-term impact of prenatal exposure to chemotherapy on executive functioning: an ERP study. Clinical Neurophysiology doi: j.clinph.2019.06.01

This article was awarded with the 2019 William Cobb Young Investigator Paper Award by the Associate Editors of Clinical Neurophysiology, and approved by the Executive Committee of the International Federation of Clinical Neurophysiology (IFCN). 


\subsection{Abstract}

This study examines the long-term impact of prenatal exposure to chemotherapy on executive functioning and the contribution of late-prematurity to this effect, using event-related potentials.

Mothers of the prenatal-exposed children $(n=20)$ were diagnosed with cancer and received chemotherapeutic treatment during pregnancy. We recruited healthy controls $(n=20)$ who were matched on a $1: 1$ ratio regarding prematurity, age and sex.

We assessed executive functioning at the age of nine, using two event-related potential paradigms: a Go/Nogo paradigm to investigate processes of response inhibition and conflict monitoring, as well as a Posner paradigm to investigate spatial attention.

Lower potentials were found in prenatal-exposed children compared to controls in the Go/Nogo P3 and Posner positive slow wave. Moreover, prenatal-exposed children responded slower on the Posner paradigm compared to controls $(p<.033)$, with more incorrect responses $(p=.023)$. In the control group, the N2 Go/Nogo wave was more pronounced in children born after a longer gestation.

This is the first study that demonstrates an effect of prenatal exposure to chemotherapy on the development of executive functioning, not limited to the effect of late-prematurity.

This study emphasizes the necessity of a long-term follow-up of prenatal-exposed children to re-inform clinical practice on the costs and benefits of late-premature induction over treatment during pregnancy. 


\subsection{Introduction}

About one in thousand pregnancies are complicated by cancer ${ }^{2}$. Multiple studies $2 ; 5 ; 6 ; 8-10 ; 183$ indicated that chemotherapy can be safely administered after the first trimester of pregnancy ${ }^{12}$ without major sequelae for the offspring. However, these studies do show a high occurrence of (late-)prematurity (48\%), as well as a high rate of children being born small for gestational age (SGA, $21 \%$ born below $10^{\text {th }}$ birthweight percentile $)^{2 ; 6 ; 10}$. Unfortunately, these studies had a limited follow-up period $(<3 y)^{6}$ or observed populations with heterogeneous ages ${ }^{5 ; 10}$. As a result, the effects of in utero exposure to cancer therapy on development past the age of three remain largely unknown, stating the need for a longer follow-up.

Chemotherapy-induced neurotoxicity has been documented in pediatric ${ }^{18 ; 27}$ and adult ${ }^{20-22}$ cancer populations. Though most chemotherapeutic agents only partially cross the placental barrier ${ }^{11}$, cancer and chemotherapy in utero could also affect neurodevelopment through indirect pathways, such as maternal nutrition ${ }^{80}$, inflammation ${ }^{184}$, stress ${ }^{128 ; 184}$ and anxiety ${ }^{185 ; 186}$, as well as other factors ${ }^{44}$. This further emphasizes the need for a long-term cognitive follow-up of children who are prenatally exposed to cancer therapy.

Literature on the direct cognitive impact of chemotherapy shows executive functioning to be especially vulnerable ${ }^{18 ; 21-23}$. Executive functioning develops from early childhood, through adolescence and into adulthood ${ }^{61}$. As previous studies investigating in utero exposure to cancer therapy did not systematically include older children ${ }^{5 ; 6 ; 10}$, longer-term effects on executive functioning have not yet been assessed.

By comparing event-related potentials (ERP) in prenatal-exposed children at the age of nine with a matched control group, the current study attempts to examine possible long-term neurocognitive impact of prenatal exposure to chemotherapy on executive functioning, and the contribution of late-prematurity to this effect.

\subsection{Methods}

\subsubsection{Participants}

This study is based on the Belgian cohort of the international follow-up study by the International Network on Cancer, Infertility, and Pregnancy (INCIP). Mothers of prenatal-exposed children $(n=20)$ were diagnosed with cancer and received chemotherapeutic treatment during pregnancy (with or without surgery), but no radiotherapy. Children in the control group, born to healthy 
mothers, were matched on a 1:1 ratio regarding gestational age (GA), age and sex, in accordance with the methodology applied by Amant et al. ${ }^{6}$. Exclusion criteria were major obstetrical and neonatal complications which possibly affect cognitive development (e.g. neonatal infections, pre-eclampsia, ... ). All children were tested between 2010 and 2018 at the age of nine years. Full scale IQ was assessed using the Wechsler Intelligence Scale for Children (WISC, version III or V) ${ }^{187 ; 188}$. The study was approved by the local ethical committee and conducted in accordance with the Declaration of Helsinki. The prenatal-exposed group overlaps partially the samples included in previous studies by Amant et al. ${ }^{5 ; 6}$ though only 1 child was previously included at the age of nine.

\subsubsection{Paradigms}

During task execution, participants were comfortably seated in front of a 19-inch screen at an approximate distance of $1 \mathrm{~m}$.

First, using a Go/Nogo paradigm, we investigated processes of response inhibition, as reflected in the central to parietal P3 wave, and conflict monitoring, as reflected in the frontal N2 wave ${ }^{95-97}$. Participants were instructed to press a button when a square (Go) appeared in the middle of the screen and suppress response when a circle (Nogo) appeared instead (both stimuli: visual angle $=1.1^{\circ}$, duration $=1.5 \mathrm{~s}$ ). Inter-stimulus time was randomly jittered between $2.5-3 \mathrm{~s}$. When there was no stimulus presented, the participants were presented with a fixation cross in the middle of the screen. All participants completed one run, containing 120 trials of which $80 \%$ were Go-trials.

Second, using the Posner paradigm ${ }^{98}$, we investigated reorienting of attention. Earlier, more lateral, P1 and N1 ERP components in this paradigm reflect visual task detection, whereas the later parietal to occipital P3 wave and positive slow wave (pSW) reflect response selection and attentional set updating ${ }^{100 ; 101}$. Children were presented during the whole paradigm with an empty box (visual angle $=2.9^{\circ}$ ) on the left and right side of the screen, with a fixation cross in the middle ( $4^{\circ}$ off-center). During the task a geometric figure (square, triangle or circle, visual angle $=1.1^{\circ}$, duration $=600 \mathrm{~ms}$ ) appeared equiprobably within one of both boxes. Children were given a button in each hand and were asked to respond with the button according to which side the figure appeared. Some trials were preceded by either an endogenous (an arrow in the middle of the screen pointing left or right, visual angle $=1.1^{\circ}$, duration $=300 \mathrm{~ms}$ ) or exogenous (one of both boxes doubling in thickness, duration $=300 \mathrm{~ms}$ ) cue, which was valid (i.e. the cue directed attention to the side of the screen on which the task stimulus later appeared) in $75 \%$ of the cases. Between each cue and task there was an equiprobable time interval of either 400 or 550 milliseconds. After each task there was a randomly jittered time interval between 2-3 seconds 
before the next cue or task appeared. Each participant completed 3 runs, each consisting of 110 trials of which 30 were not cued, 40 were preceded by an endogenous cue and 40 were preceded by an exogenous cue. Before the first run, the children completed a short practice run to ensure understanding of the task.

\subsubsection{EEG recording and analysis}

EEG signals were acquired with $31 \mathrm{Ag} / \mathrm{AgCl}$ ring electrodes (Easy Cap) placed according to the international 10-10 system at following locations: Fp1, Fp2, Fz, F3, F4, F7, F8, FC1, FC2, FC5, FC6, FT9, FT10, Cz, C3, C4, T3, T4, CP1, CP2, CP5, CP6, Pz, P3, P4, T5, T6, O1, O2, PO9, PO10. Two linked mastoid electrodes were used as a reference. Horizontal and vertical EOG was both acquired with two electrodes, respectively at the outer canthi of the eyes and above/below the left eye. A sampling frequency of $1000 \mathrm{~Hz}$ was used with an online 12 bits A/D conversion and $70 \mathrm{~Hz}$ low-pass filtering (Schwarzer Ahms 40 channels headbox). The impedance of all electrodes was set below $20 k \Omega$.

All EEG processing was performed using a combination of EEGLAB (v14.1.1) and in-house MATLAB (v2017b) scripts. All data were band-pass filtered with a bandwidth of .5-40 Hz and resampled to $500 \mathrm{~Hz}$. Automated artifact rejection based on independent component analysis (extended-ICA) was performed with the EEGLAB MARA plugin (v1.2) ${ }^{189 ; 190}$. Subsequently, EMG artifacts were automatically removed using Canonical Correlation Analysis (CCA) ${ }^{191}$. Bad channels were identified having a kurtosis over 5SD over a whole run compared to the other channels, and spherically interpolated ${ }^{192}$. Afterwards all data was epoched with regard to the onset of the task stimulus. The epoch interval was from -200 to $1000 \mathrm{~ms}$ for Go/Nogo trials and from -750 to $1000 \mathrm{~ms}$ for Posner trials. Only correct trials (correct response within 150 to $2000 \mathrm{~ms}$ ) were considered for further analysis. The baseline interval was from -200 to $0 \mathrm{~ms}$ for Go/Nogo trials and -750 to $-550 \mathrm{~ms}$ for Posner trials. The baseline average was subtracted from each epoch. Trials having a joint probability or kurtosis higher than 4 SD compared to other trials were rejected ${ }^{192}$. ERP's were constructed for each subject by averaging epochs separately for each stimulus type for Go/Nogo trials and for each combination of cue type (Endogenous, Exogenous or no cue) and cue validity (valid or invalid) for Posner trials. To increase SNR and decrease multiple testing, ERPs were averaged along electrode clusters ${ }^{96 ; 193}$. For the Go/Nogo paradigm frontal (F3, Fz, F4), central $(\mathrm{C} 3, \mathrm{Cz}, \mathrm{C} 4)$ and parietal $(\mathrm{P} 3, \mathrm{Pz}, \mathrm{P} 4)$ regions were selected and for the Posner paradigm parietal $(\mathrm{P} 3, \mathrm{Pz}, \mathrm{P} 4)$ and occipital $(\mathrm{O} 1, \mathrm{O} 2)$ regions were selected.

To visualize group differences, group average ERPs were created for each condition of each paradigm. Similarly, to visualize effects of GA within both groups, average ERPs were created for 
preterm ( $G A<37$ weeks, $n=11$ in both groups) and term ( $G A \geq 37$ weeks, $n=9$ in both groups) children in both groups separately.

\subsubsection{Statistical analysis}

Demographic data were compared between both groups (SPSS v25), using Mann-Whitney U-test for ordinal and numerical data, and Fisher's exact test for nominal data. Median response times during correct trials for each subject and trial type, as well as rates of false alarms, misses and incorrect responses were compared between prenatal-exposed and control children using Mann-Whitney U-test.

Group effects were assessed for each ERP time point of each ERP cluster, using repeated measures ANOVA (RM-ANOVA) with group and GA (defined in days) as between-subject variables. For the Posner paradigm these analyses were performed for each cue type separately. The within-subject variables for the Go/Nogo and Posner paradigms were condition (Go vs. Nogo) and cue validity (valid vs. invalid), respectively. Similarly, effects of GA were assessed for each group separately using RM-ANOVA with GA as a continuous between-subject variable. Significance was inferred at a threshold of $p<.05$ corrected for nonsphericity using Greenhouse-Geisser correction, and Bonferroni corrected for number of tested electrode clusters (i.e. 3 and 2 for Go/Nogo and Posner respectively) over a minimal consecutive time interval of $10 \mathrm{~ms}$ (5 samples) ${ }^{194}$.

\subsubsection{Data availability statement}

The anonymized data that support the findings of this study, as well as related documents, are available from the corresponding author upon reasonable request.

\subsection{Results}

\subsubsection{Demographic and medical data}

Population characteristics are shown in Table 5.1. IQ data were missing for two children of the prenatal-exposed group. A Mann-Whitney U-test indicated a slightly younger age at testing in prenatal-exposed children (median $=9.18$ years) compared to the control group (median $=9.58$ years), $p=.001$. However, the age range of both groups was similar and narrow (8.79-9.97y), reducing possible effects of aging. Maternal age was higher in the prenatal-exposed group 
( median $=34$ years) compared to the control group (median $=30$ years), $p=.009$. This can partially be explained by the higher incidence of cancer with age ${ }^{195}$. Additional information on cancer types and chemotherapy regimens administered during pregnancy is available in Table 5.2 .

\subsubsection{Behavioral performance}

Response times and correctness are summarized in Table 5.3. For the Go/Nogo paradigm we observed no significant between-group differences on any response measure. However, in the Posner paradigm, median response times were delayed in the prenatal-exposed group for all cued conditions $(p<.033)$ and this group also had more incorrect responses (Median .9\% compared to $.3 \%$ in the control group, $p=.023)$. However, the number of incorrect responses and misses remained relatively low in both paradigms $(<2.6 \%)$ with only a slightly elevated false alarm rate in the Go/Nogo paradigm (median of $10.4 \%$ and $12.5 \%$ respectively for the prenatal-exposed and control group).

\subsubsection{ERP results}

The amount of data, per subject and condition, that was excluded due to artifacts, remained low in Posner (median $\leq 1.11 \%,[0 \%-\leq 6.6 \%]$ for all conditions) and Nogo (median=0\%, [0\%-8.33\%]) trials. This was slightly elevated in the Go-trials (median $=13.54 \%,[7.29 \%-20,83 \%]$ ), which can be explained by motion often coinciding with the go-response. None of the Channels later used in analyses had to be interpolated for the Go/Nogo paradigm and occurred only rarely in Posner runs ( median $=0 / 5$ and maximum $2 / 5$ channels per run).

Grand averages and significant group differences in time for the Go/Nogo paradigm are depicted in Figure 5.1 and Table 5.4. Significant group effects (prenatal-exposed versus control group) are found centrally (456-546ms) and parietally (456-574ms, 638-648ms and 942-958ms), both indicating lower amplitudes in prenatal-exposed children compared to controls, mostly overlapping the P3 wave. No significant group-by-condition interactions were found. In the prenatal-exposed group two early main effects of GA were found frontally (164-182ms) and parietally (52-82ms). Lower/more negative values were observed with longer GA in the control group for both frontal (436-490ms) and central (438-488ms) clusters, which coincides with the later part of the N2 wave. Additionally, in this group, a late interaction effect of GA by condition was observed parietally (874-914ms). 
Table 5.1 - Population characteristics. Continuous parameters are reported with median and range per group, whereas frequencies are reported with the absolute count as well as the percentage. Ordinal and numeric parameters were compared between both groups using Mann-Whitney U-test and categorical parameters using Fisher's exact test (underlined). Significant results $(p<.05$, uncorrected) are indicated in bold. Min: minimum. Max: maximum

\begin{tabular}{|c|c|c|c|c|c|c|c|}
\hline & \multicolumn{3}{|c|}{ Prenatal-exposed group } & \multicolumn{3}{|c|}{ Control group } & \multirow{2}{*}{$\begin{array}{c}\text { statistics } \\
\mathrm{p}\end{array}$} \\
\hline & Median & Min & Max & Median & Min & Max & \\
\hline Age (years) & 9.18 & 8.79 & 9.94 & 9.58 & 9.07 & 9.97 & .001 \\
\hline GA (weeks+days) & $35+6$ & $28+3$ & $40+1$ & $36+0$ & $28+2$ & $40+0$ & .799 \\
\hline Birth weight (g) & 2903 & 1405 & 3905 & 2793 & 940 & 3410 & .565 \\
\hline Maternal age at birth (years) & 34 & 25 & 40 & 30 & 26 & 39 & .009 \\
\hline \multirow{2}{*}{ Total IQ } & 106 & 74 & 130 & 109 & 62 & 128 & .149 \\
\hline & Count & $\%$ & & Count & $\%$ & & $\mathrm{P}$-value \\
\hline $\mathrm{N}$ & 20 & & & 20 & & & \\
\hline Twins & 2 & $10 \%$ & & 2 & $10 \%$ & & 1 \\
\hline Corrected vision & 8 & $40 \%$ & & 6 & $30 \%$ & & .523 \\
\hline Sex & & & & & & & 1 \\
\hline Male & 9 & $45 \%$ & & 9 & $45 \%$ & & \\
\hline Female & 11 & $55 \%$ & & 11 & $55 \%$ & & \\
\hline Ethnicity & & & & & & & .113 \\
\hline Caucasian & 17 & $85 \%$ & & 17 & $85 \%$ & & \\
\hline Asian & 1 & $5 \%$ & & 0 & $0 \%$ & & \\
\hline African & 2 & $10 \%$ & & 0 & $0 \%$ & & \\
\hline Mixed & 0 & $0 \%$ & & 3 & $15 \%$ & & \\
\hline Handedness & & & & & & & .695 \\
\hline Right & 15 & $75 \%$ & & 17 & $85 \%$ & & \\
\hline Left & 5 & $25 \%$ & & 3 & $15 \%$ & & \\
\hline \multicolumn{8}{|l|}{ Level of education parents } \\
\hline Mother & & & & & & & .583 \\
\hline Primary school & 1 & $5 \%$ & & 0 & $0 \%$ & & \\
\hline Secondary school & 8 & $40 \%$ & & 3 & $15 \%$ & & \\
\hline Bachelor & 3 & $15 \%$ & & 12 & $60 \%$ & & \\
\hline Master & 8 & $40 \%$ & & 5 & $25 \%$ & & \\
\hline Father & & & & & & & .355 \\
\hline Primary school & 1 & $5 \%$ & & 0 & $0 \%$ & & \\
\hline Secondary school & 9 & $45 \%$ & & 5 & $25 \%$ & & \\
\hline Bachelor & 3 & $15 \%$ & & 8 & $40 \%$ & & \\
\hline Master & 7 & $35 \%$ & & 7 & $35 \%$ & & \\
\hline Smoking or drugs during pregnancy & 0 & $0 \%$ & & 0 & $0 \%$ & & 1 \\
\hline Alcohol during pregnancy & & & & & & & 1 \\
\hline Yes & 2 & $10 \%$ & & 1 & $5 \%$ & & \\
\hline Unknown & 1 & $5 \%$ & & 1 & $5 \%$ & & \\
\hline
\end{tabular}


Table 5.2 - Maternal tumor types and treatment during pregnancy. Chemotherapy regimen and number of cycles are mentioned as administered during pregnancy. FEC: 5-fluorouracil, epirubicin and cyclophosphamide. FAC: 5-fluorouracil, doxorubicin (adiamycin) and cyclophosphhamide. AC: doxorubicin and cyclophosphamide. ABVD: doxorubicin, Bleomycin, Vinblastine, Dacarbazine. Ara-C: cytarabine. R-CHOP: rituximab, cyclophosphamide, doxorubicin, vincristine and prednisolone. Min: minimum. Max: maximum

\begin{tabular}{|c|c|c|c|c|c|}
\hline Maternal disease & \multicolumn{2}{|l|}{ Count } & & & \\
\hline Breast cancer & 12 & $60 \%$ & & & \\
\hline Cervical cancer & 2 (twin) & $10 \%$ & & & \\
\hline Hodgkin lymphoma & 2 & $10 \%$ & & & \\
\hline Non-Hodgkin lymphoma & 1 & $5 \%$ & & & \\
\hline Acute lymphoblastic leukemia & 1 & $5 \%$ & & & \\
\hline Acute myeloid leukemia & 1 & $5 \%$ & & & \\
\hline Colon carcinoma & 1 & $5 \%$ & & & \\
\hline \multirow[t]{2}{*}{ Chemotherapy during pregnancy } & & & \multicolumn{3}{|c|}{ Cycles } \\
\hline & & & Median & Min & Max \\
\hline FEC/FAC & 7 & $35 \%$ & 6 & 2 & 6 \\
\hline$A C$ & 3 & $15 \%$ & 4 & 3 & 5 \\
\hline ABVD & 2 & $10 \%$ & 3 & 2 & 4 \\
\hline Neo-adjuvant platinum & 2 (twin) & $10 \%$ & 3 & & \\
\hline 5-fluorouracil & 1 & $5 \%$ & 3 & & \\
\hline Ara- $C$, Vincristine and mitoxantrone & 1 & $5 \%$ & 2 & & \\
\hline Cyclophosphamide + methotrexate & 1 & $5 \%$ & 1 & & \\
\hline Daunorubicin/cytarabine & 1 & $5 \%$ & 2 & & \\
\hline $\mathrm{R}-\mathrm{CHOP}$ & 1 & $5 \%$ & 1 & & \\
\hline Trastuzumab & 1 & $5 \%$ & $13-18$ & & \\
\hline
\end{tabular}


Table 5.3 - Behavioral performance on both paradigms. Median, minimum and maximum response time, for correct responses, is summarized for both groups, as well as number of missed and incorrect responses. Response times are calculated per subject as their median response time. A miss is defined as no response or a response before $150 \mathrm{~ms}$ or after 2s. The effect of group on each parameter is assessed using Mann-Whitney U-test. Significant results $(p<.05$, uncorrected) are indicated in bold. GA: gestational age. Min: minimum. Max: maximum

\begin{tabular}{|c|c|c|c|c|c|c|c|c|}
\hline & \multicolumn{3}{|c|}{ Prenatal-exposed group } & \multicolumn{3}{|c|}{ Control group } & \multirow{2}{*}{$\begin{array}{c}\text { group effect } \\
p\end{array}$} \\
\hline & & median & $\min$ & $\max$ & median & $\min$ & $\max$ & \\
\hline \multicolumn{9}{|l|}{ Go/Nogo } \\
\hline \multicolumn{2}{|c|}{ Incorrect (\%) } & $10.4 \%$ & $4.2 \%$ & $37.5 \%$ & $12.5 \%$ & $0 \%$ & $29.2 \%$ & .640 \\
\hline \multicolumn{2}{|c|}{ Miss ( $\%)$} & $.5 \%$ & $0 \%$ & $14.6 \%$ & $0 \%$ & $0 \%$ & $9.4 \%$ & .211 \\
\hline \multicolumn{2}{|c|}{ Response time (ms) } & 562 & 406 & 763 & 534 & 429 & 739 & .149 \\
\hline \multicolumn{9}{|l|}{ Posner } \\
\hline \multicolumn{2}{|c|}{ Miss $(\%)$} & $2.6 \%$ & $0 \%$ & $7.6 \%$ & $.9 \%$ & $0 \%$ & $10.4 \%$ & .056 \\
\hline \multicolumn{2}{|c|}{ Incorrect (\%) } & $.9 \%$ & $0 \%$ & $9.1 \%$ & $.3 \%$ & $0 \%$ & $2.1 \%$ & .023 \\
\hline \multicolumn{9}{|c|}{ Response time (ms): } \\
\hline \multirow{3}{*}{ Exogenous cue } & No cue & 534 & 459 & 665 & 501 & 404 & 611 & .076 \\
\hline & Valid & 429 & 356 & 625 & 399 & 335 & 481 & .033 \\
\hline & Invalid & 519 & 450 & 654 & 485 & 369 & 541 & .005 \\
\hline \multirow{2}{*}{ Endogenous cue } & Valid & 421 & 362 & 630 & 392 & 312 & 457 & .023 \\
\hline & Invalid & 490 & 408 & 698 & 439 & 375 & 519 & .003 \\
\hline
\end{tabular}

Figures 5.2 and 5.3 show the grand averages of the Posner ERPs, respectively for the parietal and occipital clusters. The significant differences in time are detailed in Table 5.4. Main effects of group, with lower amplitudes in the prenatal-exposed group compared to controls, were observed: parietally for the exogenous cued (204-216ms) and uncued (472-568ms) conditions, and occipitally for the exogenous cued (452-548ms), endogenous cued (454-492ms) and uncued (522-538ms and 550-556ms) conditions, all but the first overlapping the pSW. Main effects of GA within the prenatal-exposed group were observed both parietally for the exogenous cued condition (430-456ms) and occipitally for the exogenous (414-472ms and 692-742ms) and uncued (604-624ms and 666-694ms) conditions, all indicating a more positive amplitude with higher prematurity in the $\mathrm{pSW}$. In the same group, interaction effects of GA by cue validity were found parietally for the exogenous cued condition (866-900ms) and occipitally for both exogenous (390-408ms, 426-470ms, 706-732ms and 864-900ms) and endogenous (508-528ms and $860-888 \mathrm{~ms}$ ) cued conditions, all indicating higher potentials for the incorrect compared to correct cued trials with higher prematurity. All but the last cluster were located within the pSW. One early parietal effect of GA in the control group was observed in the exogenous cued condition (130-150ms). 

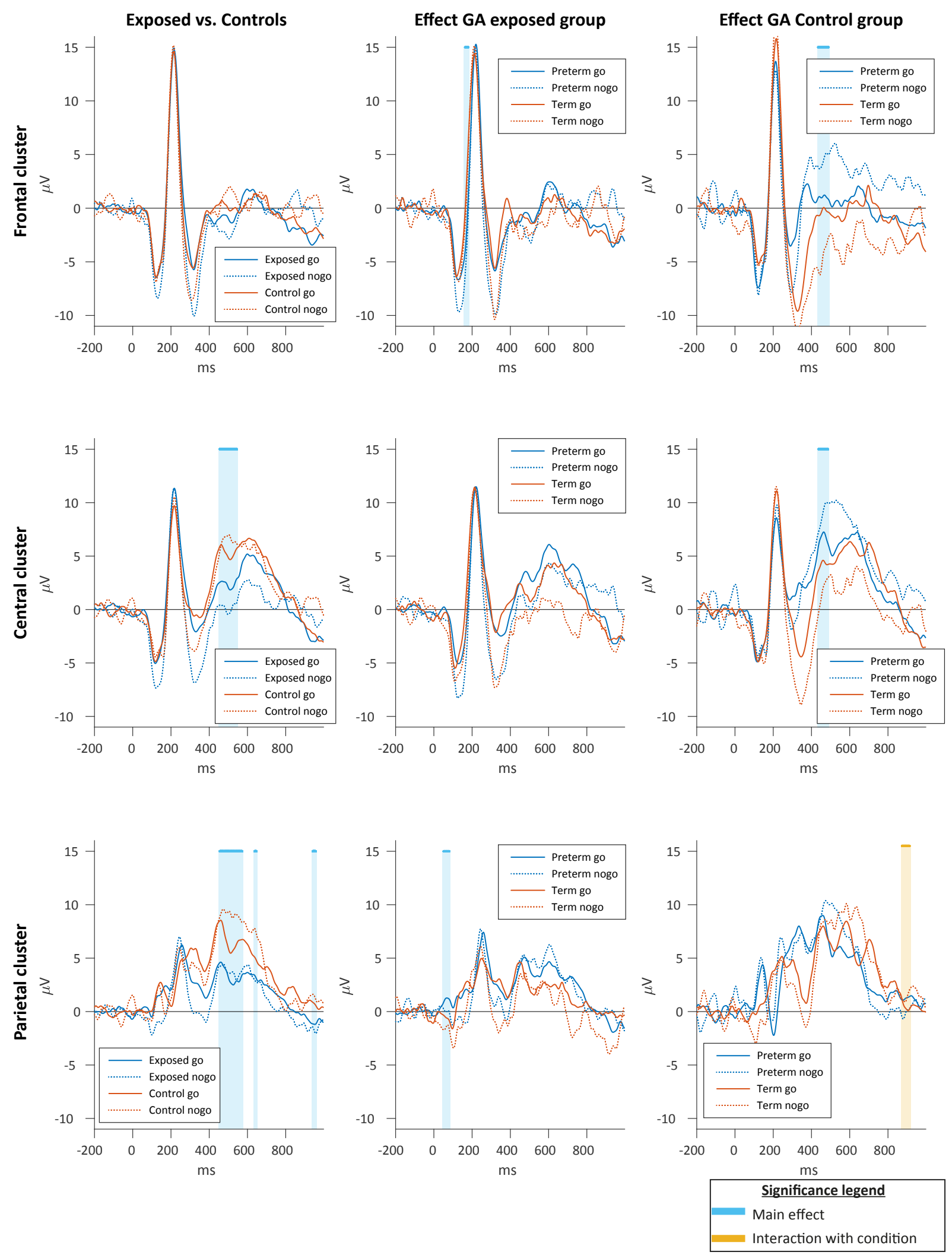

Figure 5.1 - Event-related potentials of the Go/Nogo paradigm. Left column shows the average responses in the 3 electrode clusters of interest (frontal, central and parietal, respectively in rows 1,2 and 3) for both prenatal-exposed and control groups. Significant differences $p<.05$. Bonferroni corrected for 3 clusters, min. $10 \mathrm{~ms}$ ) between both groups, are highlighted in blue, and significant interaction effects of group (i.e. exposed/controls) by condition (i.e. go/nogo) in yellow. The middle and right column show the average responses, respectively in the prenatal-exposure and control groups, for the preterm and term children. Significant differences with gestational age are highlighted in blue, and significant interaction effects of gestational age by condition (i.e. go/nogo) in yellow. 
Table 5.4 - List of all significant time intervals in the event-related potentials of both paradigms. GA: Gestational age

\begin{tabular}{|c|c|c|c|}
\hline $\begin{array}{r}\text { Electrode } \\
\text { cluster }\end{array}$ & Cue type & Contrast & $\begin{array}{l}\text { Significant time } \\
\text { interval }[\mathrm{ms}]\end{array}$ \\
\hline \multicolumn{4}{|l|}{ Go/Nogo } \\
\hline \multirow[t]{2}{*}{ Frontal } & & Linear positive main effect of GA in the exposed group & $164-182 \mathrm{~ms}$ \\
\hline & & Linear negative main effect GA in the control group & 436-490ms \\
\hline \multirow[t]{2}{*}{ Central } & & Main effect of exposed < control group & $456-546 \mathrm{~ms}$ \\
\hline & & Linear negative main effect of GA in the control group & $438-488 \mathrm{~ms}$ \\
\hline \multirow[t]{3}{*}{ Parietal } & & Main effect of exposed $<$ control group & $\begin{array}{c}456-574 \mathrm{~ms}, 638-648 \mathrm{~ms}, \\
942-958 \mathrm{~ms}\end{array}$ \\
\hline & & Linear negative main effect of GA in the exposed group & $52-82 \mathrm{~ms}$ \\
\hline & & Interaction GA by cue validity in the control group & $874-914 \mathrm{~ms}$ \\
\hline \multicolumn{4}{|l|}{ Posner } \\
\hline \multirow[t]{13}{*}{ Parietal } & Exogenous & Main effect of exposed $<$ control group & $204-216 \mathrm{~ms}$ \\
\hline & & Linear negative main effect of GA in the exposed group & $430-456 \mathrm{~ms}$ \\
\hline & & Interaction GA by cue validity in the exposed group & $864-900 \mathrm{~ms}$ \\
\hline & & Linear negative main effect of GA in the control group & $130-150 \mathrm{~ms}$ \\
\hline & No & Main effect of exposed $<$ control group & $472-568 \mathrm{~ms}$ \\
\hline & Occipital & Exogenous Main effect of exposed $\mathrm{i}$ control group & $452-548 \mathrm{~ms}$ \\
\hline & & Linear negative main effect of $\mathrm{GA}$ in the exposed group & $412-472 \mathrm{~ms}, 692-742 \mathrm{~ms}$ \\
\hline & & Interaction GA by cue validity in the exposed group & $390-408 \mathrm{~ms}, 426-470 \mathrm{~ms}$, \\
\hline & & & $706-732 \mathrm{~ms}, 864-900 \mathrm{~ms}$ \\
\hline & Endogenous & Main effect of exposed < control group & $454-492 \mathrm{~ms}$ \\
\hline & & Interaction GA by cue validity in the exposed group & $508-528 \mathrm{~ms}, 860-888 \mathrm{~ms}$ \\
\hline & No & Main effect of exposed $<$ control group & $522-538 \mathrm{~ms}, 550-566 \mathrm{~ms}$ \\
\hline & & Linear negative main effect of GA in the exposed group & $604-624 \mathrm{~ms}, 666-694 \mathrm{~ms}$ \\
\hline
\end{tabular}



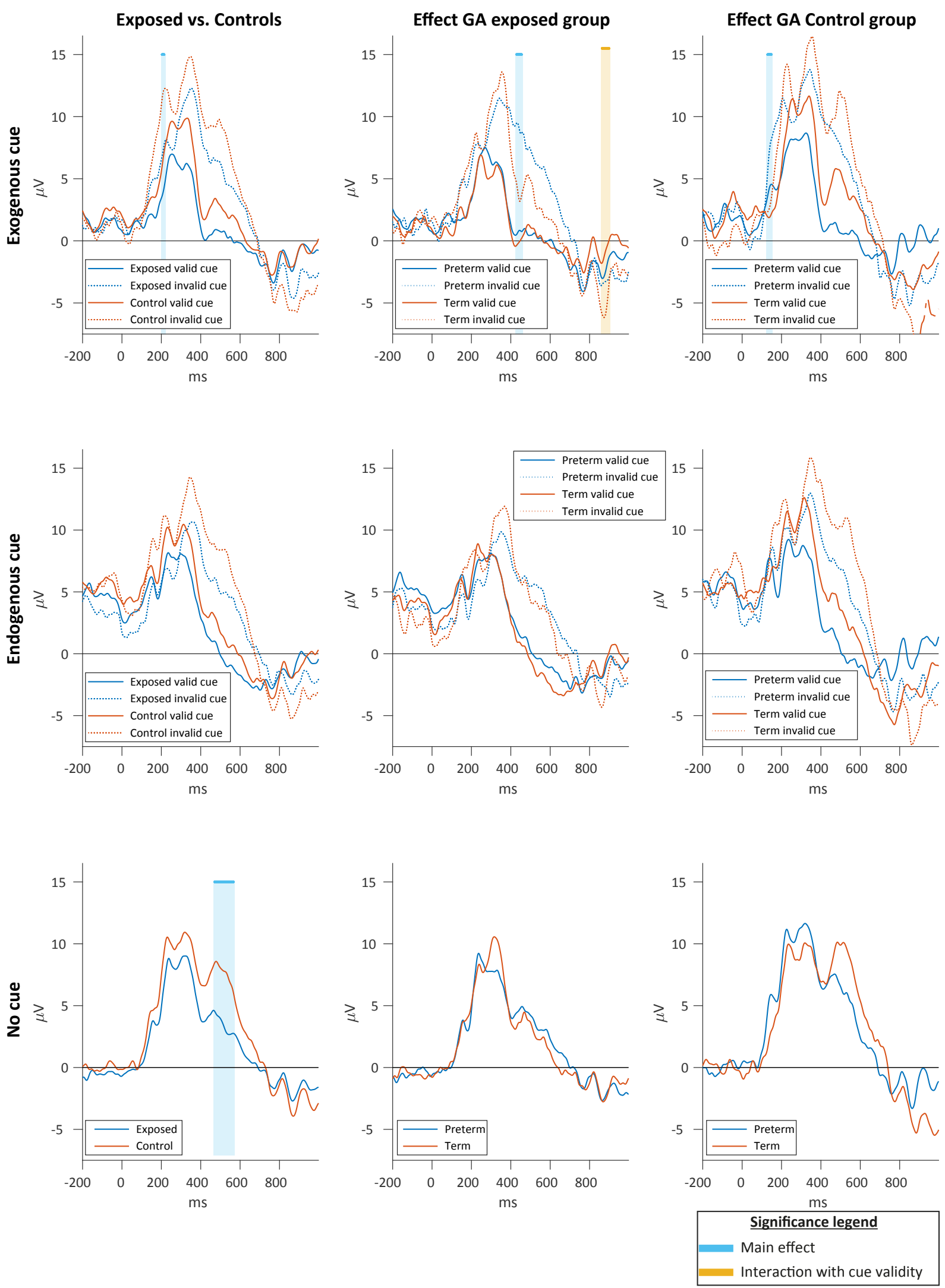

Figure 5.2 - Parietal event-related potentials of the Posner paradigm. Left column shows the average responses of each cue type (exogenous, endogenous and no cue, respectively in rows 1,2 and 3) for both prenatal-exposed and control groups in the parietal electrode cluster. Significant differences $(p<.05$, Bonferroni corrected for 2 clusters, min. $10 \mathrm{~ms}$ ) between both groups, as indicated by RM-ANOVA, are highlighted in blue, and significant interaction effects of group (i.e. exposed/controls) by cue validity in yellow. The middle and right column show the average responses, respectively in the prenatal-exposure and control groups, for the preterm and term. Significant differences with gestational age, as indicated by RM-ANOVA, are highlighted in blue, and significant interaction effects of gestational age by cue validity in yellow. 

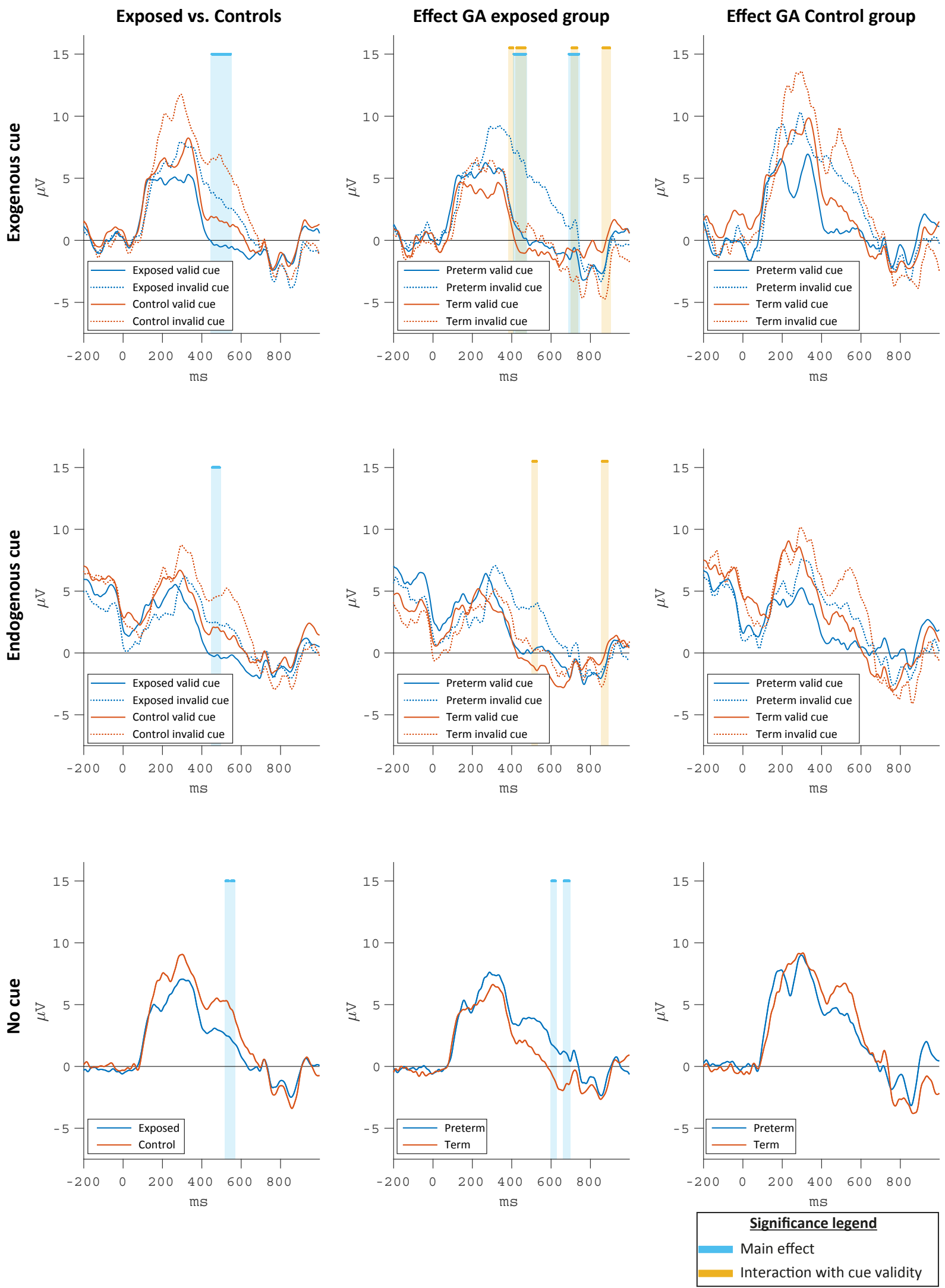

Figure 5.3 - Occipital event-related potentials of the Posner paradigm. Left column shows the average responses of each cue type (exogenous, endogenous and no cue, respectively in rows 1,2 and 3) for both prenatal-exposed and control groups in the occipital electrode cluster. Significant differences $(p<.05$, Bonferroni corrected for 2 clusters, min. 10ms) between both groups, as indicated by RM-ANOVA, are highlighted in blue, and significant interaction effects of group (i.e. exposed/controls) by cue validity in yellow. The middle and right column show the average responses, respectively in the prenatal-exposure and control groups, for the preterm and term children. Significant differences with gestational age, as indicated by RM-ANOVA, are highlighted in blue, and significant interaction effects of gestational age by cue validity in yellow. 


\subsection{Discussion}

This study investigated the impact of prenatal exposure to chemotherapy on the development of executive functioning, and the contribution of late-prematurity to this effect, through the use of ERP. In the Go/Nogo paradigm (Figure 5.1 and Table 5.4), lower P3 amplitudes were found in central and parietal clusters for prenatal-exposed children, compared to controls, indicating an effect on response inhibition. Using the same paradigm, shorter gestation at birth in the control group was correlated with a less negative signal in the late fronto-central N2 component, indicating an effect of late-prematurity on conflict monitoring. In the Posner paradigm (Figures 5.2,5.3 and Table 5.4), lower pSW amplitudes were found in the prenatal-exposed group compared to controls, indicating an impact on cognitive resources that are required for response selection and cognitive model updating. Moreover, this outcome was further empowered by a slower response time to all cued conditions, as well as a higher incorrect rate in the prenatal-exposed group compared to controls. Within the prenatal-exposed group GA negatively correlated with pSW amplitudes as well as larger discrimination in the pSW amplitude between valid and invalid cued trials. Additionally, in the Posner paradigm, an early effect was found for prenatal-exposed children compared to control children, as well as with prematurity in the prenatal-exposed group. Though these effects could indicate changes in task detection, these results should be carefully interpreted as these early components are highly affected by the more pronounced P3 component.

The lower Go/Nogo P3 amplitude, observed in the prenatal-exposed group, has similarly been observed by Boucher et al. after prenatal exposure to tobacco ${ }^{68}$. This P3 wave is related to response inhibition and cognitive resources for task execution ${ }^{95-97}$. On the other hand Boucher et al. found both prenatal exposure to tobacco and childhood exposure to several contaminants to be correlated with a less negative $\mathrm{N} 2{ }^{66 ; 68}$, which is related to conflict monitoring. Here we only observed a more negative late N2 peak with higher GA in control children, indicating that both prematurity and prenatal exposure might have an effect on conflict monitoring.

In the Posner paradigm prenatal-exposed children demonstrated delayed response times, more incorrect responses, and a lower pSW amplitude, which all indicate an effect of prenatal exposure to chemotherapy on spatial attention. Reorientation of spatial attention is an essential process for developing cognitive flexibility and creative thinking ${ }^{61}$. Hence, development of these cognitive skills might be impacted as well.

Within the prenatal-exposed group, the pSW in the Posner paradigm showed to be further lowered with longer gestation. Moreover, the observed difference in pSW amplitude between valid and invalidly cued conditions was smaller in this group with longer gestation. However, this effect 
of prematurity is not observed in the control group, indicating that this observation is not directly related to prematurity. Rather, this observation might result from other effects, such as differences in cumulative chemotherapy doses, chemotherapy types and treatment timing with regards to the fetal development. Similarly, cancer diagnosis can induce stress and anxiety, impacting fetal neurodevelopment. This impact of prenatal stress on neurodevelopment has earlier been found to be dependent on its timing during pregnancy ${ }^{75}$. While current literature observed a higher incidence of prematurity and SGA after prenatal exposure to cancer therapy, cancer therapy during pregnancy has not been found to impact the neurodevelopment of these children ${ }^{5 ; 6 ; 10}$. However, these studies focused on IQ as an indicator for neurodevelopment, which was also not found to be significantly different between groups in this study ( $p=.15$ ). To our knowledge, this study is the first to investigate a link between prenatal exposure to chemotherapy and the development of executive functioning. This suggests that although prenatal exposure to cancer treatment is not necessarily observed in IQ-scores obtained early in life ${ }^{5 ; 6 ; 10}$, specific cognitive skills which develop later in childhood and adolescence, such as executive functioning, might be affected in later life.

Neuropsychological studies demonstrated preterm birth to affect executive functioning later in life, including processes of inhibition and cognitive flexibility ${ }^{72}$. In this study we observed an effect of prematurity on conflict monitoring in a Go/Nogo experiment, but not on spatial attention. However, 15/22 premature children, included in current study, are born moderate to late preterm, which remains an understudied population in current literature ${ }^{72}$. Moreover, several common comorbidities of preterm born children, which were explicitly excluded in the current study, might partially explain the deviation of our findings from previous research. Both these factors and the limited sample size of the current study might explain the more subtle nature of the observed findings regarding prematurity.

Current guidelines in oncological treatment during pregnancy advise to, whenever feasible, treat cancer during pregnancy in favor of a longer gestation ${ }^{12}$. However, this study found response inhibition and spatial attention to be affected in children that were prenatally exposed to chemotherapy, while this was not correlated to (late-)prematurity. These findings, on their own, are not a contra-indication for chemotherapy administration during pregnancy. However, it does indicate that further research is necessary on the long-term impact of chemotherapy during pregnancy to re-inform clinical practice on the costs and benefits of late-premature induction over treatment during pregnancy.

Some limitations in this study should be mentioned. First, cancer in pregnancy is a heterogenous pathology, resulting in a heterogeneous treatment. As a consequence, this study cannot discriminate whether some cancer pathologies and treatments contribute more to the observed 
effects. Furthermore, cancer diagnosis and treatment during pregnancy might affect neurocognitive development through a multitude of direct and indirect pathways ${ }^{44}$. The current study cannot discriminate the contributions of individual pathways to the observed effect. However, by excluding major obstetrical and neonatal complications, we try to control for effects on maternal health that are not caused by the cancer diagnosis or treatment. Next, due to the relative rare occurrence of cancer in pregnancy, we acquired data during a relative long period of time and only recruited 20 prenatal-exposed children. However, the testing set-up remained unchanged during the whole testing period. Moreover, we used a priori knowledge from literature to limit multiple testing and age effects were limited by testing at a very narrow age-range (8.79-9.91y).

\subsection{Conclusions}

To our knowledge, this study is the first to find an effect of prenatal exposure to chemotherapy on the development of executive functioning, which was not limited to the effect of late-prematurity. This further emphasizes the need for a long-term follow-up of prenatal-exposed children, as these cognitive functions will further develop throughout adolescence ${ }^{61}$. Furthermore, multi-disciplinary and multi-modal approaches will be essential in understanding the neurocognitive extent and neurobiological substrate of prenatal exposure to chemotherapy. Though the observed effects in this study are relatively subtle, the authors believe this will further guide clinical decision-making and will enable physicians to better inform patients, eventually contributing to a better treatment and follow-up of cancer in pregnancy. 


\title{
CHAPTER
}

\author{
6
}

\section{THE IMPACT OF CANCER AND CHEMOTHERAPY} DURING PREGNANCY ON CHILD NEURODEVELOPMENT: A MULTIMODAL NEUROIMAGING ANALYSIS

This chapter has been accepted for publication as:

Blommaert J., Radwan A., Sleurs C., Maggen C., van Gerwen M., Wolters V. Christiaens D., Peeters R., Dupont P., Sunaert S., Van Calsteren K., Deprez S. and Amant F. (accepted). The impact of cancer and chemotherapy during pregnancy on child neurodevelopment: a multimodal neuroimaging analysis, EClinicalMedicine 


\subsection{Abstract}

This study applies multimodal MRI to investigate neurodevelopment in nine-year-old children born to cancer-complicated pregnancies.

In this cohort study, children born after cancer-complicated pregnancies were recruited alongside 1:1 matched controls regarding age, sex and gestational age at birth (GA). Multimodal MRI was used to investigate whole-brain and subcortical volume, cortical structure (using surface-based morphometry), white matter microstructure (using fixel-based analysis) and functional connectivity (using resting-state blood-oxygen-level-dependent signal correlations). Graph theory probed whole-brain structural and functional organization. For each imaging outcome we conducted two group comparisons: 1) children born after cancer-complicated pregnancies versus matched controls, and 2) the subgroup of children with prenatal chemotherapy exposure versus matched controls. In both models, we used the covariate of GA and the group-by-GA interaction, using false-discovery-rate (FDR) or family-wise-error (FWE) correction for multiple comparisons. Exploratory post-hoc analyses investigated the relation between brain structure/function, neuropsychological outcome and maternal oncological/obstetrical history.

Forty-two children born after cancer-complicated pregnancies were included in this study, with 30 prenatally exposed to chemotherapy. Brain organization and functional connectivity were not significantly different between groups. Both cancer and chemotherapy in pregnancy, as compared to matched controls, were associated with a lower travel depth, indicating less pronounced gyrification, in the left superior temporal gyrus $\left(p_{F D R} \leq .006\right)$, with post-hoc analysis indicating platinum derivatives during pregnancy as a potential risk factor $(p=.028)$. Both cancer and chemotherapy in pregnancy were related to a lower fibre cross-section (FCS) and lower fibre density and cross-section (FDC) in the posterior corpus callosum and its tapetal fibres, compared to controls. Higher FDC in the chemotherapy group and higher FCS in the whole study group were observed in the anterior thalamic radiations. None of the psycho-behavioral parameters correlated significantly with any of the brain differences in the study group or chemotherapy subgroup.

Prenatal exposure to maternal cancer and its treatment might affect local grey and white matter structure, but not functional connectivity or global organization. While platinum-based therapy was identified as a potential risk factor, this was not the case for chemotherapy in general. 


\subsection{Introduction}

Cancer complicates about one in 1000 pregnancies $^{2}$. This diagnosis unavoidably leads to difficult medical and ethical decisions ${ }^{1}$. While treatment delay can worsen the maternal prognosis, starting cancer treatment during pregnancy and preterm delivery might impact foetal development. Over the last twenty years, evidence of short-term safety of cancer treatment during pregnancy has been growing $2 ; 5-7 ; 10 ; 17$ resulting in an increased number of mothers being treated during their pregnancy and less pregnancy terminations, iatrogenic preterm deliveries and treatment delays ${ }^{2}$.

Chemotherapy is contraindicated before 12 weeks of pregnancy due to the increased risk of congenital anomalies ${ }^{12}$. Chemotherapy exposure in the second and third trimester of pregnancy has been associated with more growth restriction and preterm delivery ${ }^{2}$. Moreover, the impact of chemotherapy on neurocognitive development remains a concern as the foetal brain is rapidly developing during the second and third trimesters of pregnancy through processes of neurogenesis, neuronal migration, synaptogenesis, etc. ${ }^{45 ; 46}$. One mouse study observed prenatal exposure to vinblastine and doxorubicin to affect brain development, impacting both brain structure and behaviour ${ }^{15}$. Such early life impact might only become apparent in later life as the child develops into adolescence ${ }^{62}$ and adulthood ${ }^{45}$.

To date, prenatal exposure to cancer treatment does not appear to be associated with altered neurocognitive development in children aged up to three years old $5 ; 6 ; 10 ; 17$. However, the associated frequent prematurity has been identified as an important risk factor for lower cognitive scores in early childhood ${ }^{5-7 ; 10}$. Later during childhood however, there are indications that prenatal chemotherapy exposure might be linked to more internalizing and externalizing behavioural problems ${ }^{10}$, as well as a lower performance on verbal intelligence ${ }^{14}$ and executive functioning tasks ${ }^{196}$.

In multiple cancer populations, chemotherapy has been associated with altered neurocognition, as well as both structural and functional brain changes ${ }^{20}$. However, when assessing the neurocognitive impact of prenatal cancer treatment exposure, secondary effects such as low birth weight, prematurity and maternal malnutrition, depression, stress and anxiety might also impact neurodevelopment ${ }^{44}$. It has been hypothesized that the observed impact on psycho-behavioural development of the offspring, might be partially explained by the psychosocial impact of a cancer diagnosis on the mother during pregnancy ${ }^{14 ; 63 ; 82}$.

State-of-the-art multimodal magnetic resonance imaging (MRI) of the brain has proven to be a valuable tool in characterizing and understanding impaired and healthy neurodevelopment. In this study, we employ state-of-the-art multimodal MRI techniques, in combination with psychological 
testing, as well as obstetrical and oncological parameters, to increase understanding of the potentially detrimental effects of cancer and its treatment during pregnancy on structural and functional brain development in the offspring at nine years old.

\subsection{Methods}

\subsubsection{Participants}

This cohort study prospectively included children of the Belgian cohort from the international follow-up study of the International Network on Cancer, Infertility, and Pregnancy (INCIP) ${ }^{197}$. Children in the study group were born to mothers with a cancer diagnosis during pregnancy. Children in the control group, born to healthy mothers, were matched on a 1:1 ratio regarding gestational age at birth (GA, maximum 1 week difference), age (9 years old) and sex. Participant recruitment is further detailed by van Gerwen et al. ${ }^{198}$. Exclusion criteria in both groups were major obstetrical and neonatal complications which possibly affect cognitive development (e.g. neonatal infections, pre-eclampsia), which was checked through medical records and parents-reported questionnaires on prenatal history and general health. All children were tested between 2015 and 2020 at the age of nine years, at the university hospital of Leuven, Belgium. This sample partially overlaps with the samples in our previous research $5 ; 6 ; 14 ; 196$, though assessments in these cohort studies were often performed at different ages and never included any MRI-derived measures.

Full scale intelligence was assessed by a psychologist, using the Wechsler Intelligence Scale for Children (WISC, version III or V) ${ }^{187 ; 188}$. The WISC-IV was not used as it was never translated to dutch, with the WISC-V being introduced in 2018, replacing the WISC-III.Verbal intelligence of children was included as an outcome parameter when the WISC III was used. The Child Behavior Checklist (CBCL) was used to assess behavioural development through three scales of internalizing, externalizing and total behavioural problems ${ }^{199}$. The Behavior Rating Inventory of Executive Function (BRIEF) ${ }^{200}$ was used to assess executive functioning through three composite scores: behavioral regulation, metacognition and the global executive composite score.

Data on oncological treatment, obstetrical outcome and demographics, were collected via the INCIP registry as described elsewhere ${ }^{2}$. Customized birth weight percentiles were calculated using the BULK GROW (v8.0.4, 2019) calculator, adjusting for nationality, maternal height/weight, parity, sex and $\mathrm{GA}^{201}$.

The study was approved by the local ethical committee and conducted in accordance with the 
Declaration of Helsinki. This study is registered at ClinicalTrials.gov: NCT00330447. Informed consent was signed by one of the parents or legal guardians upon participation. The full study protocol is available at http://www.cancerinpregnancy.org/study-protocols.

\subsubsection{MRI acquisition}

All children underwent a half-hour whole-brain MRI scanning protocol, using the same scanner (3T Philips Achieva, 32-channel phased-array head coil). To avoid subject motion, children were familiarized with the scanner prior to entering the scanner room, were repeatedly encouraged not to move and a movie was presented during all non-functional images. Multiple MRI modalities were acquired: T2-weighted fluid-attenuated inversion recovery images (T2-FLAIR, resolution $=0.68 \times 0.68 \times 4 \mathrm{~mm}$, $1 \mathrm{~mm}$ slice gap, $\left.\mathrm{TR} / \mathrm{TE} / \mathrm{TI}=9000 / 120 / 2500 \mathrm{~ms}, \mathrm{FA}=90^{\circ}, \mathrm{FOV}=230 \times 139 \times 187 \mathrm{~mm}\right)$, high-resolution T1-weighted images (MPRAGE, resolution $=.98 \times .98 \times 1.2 \mathrm{~mm}, \mathrm{TR} / \mathrm{TE}=9.6 / 4.6 \mathrm{~ms}, \mathrm{FA}=8^{\circ}$, $F O V=160 \times 256 \times 256 \mathrm{~mm}$ ), multi-shell diffusion-weighted images ( $b$-value $=0 / 700 / 2000 \mathrm{~s} / \mathrm{mm}^{2}$ with respectively $6 / 30 / 60$ uniformly distributed gradient directions, resolution $=2.5 \times 2.5 \times 2.5 \mathrm{~mm}$, $F O V=240 \times 240 \times 125 \mathrm{~mm}, T R / T E=7000 / 72 \mathrm{~ms}, F A=90^{\circ}$, Phase encoding $=A P$, halfscan $=0.766$, one additional b0 image with reversed phase-encoding) and resting-state functional images (rs-fMRI, using T2*-weighted Echo-planar imaging, resolution $=3.59 \times 3.59 \times 4 \mathrm{~mm}, F O V=230 \times 230 \times 120 \mathrm{~mm}$, $\mathrm{FA}=90^{\circ}, \mathrm{TE} / \mathrm{TR}=33 / 1700 \mathrm{~ms}$, acquisition time $=7 \mathrm{~min}, 250$ volumes +4 initial dummy volumes).

\subsubsection{Data Analysis}

A detailed overview of all analysis steps performed can be found in the supplementary materials. All analyses included multiple steps of bias, motion and artefact correction ${ }^{106 ; 108 ; 109 ; 153 ; 202-204}$, as well as visual and quantitative quality assurance ${ }^{109 ; 112}$.

First, T2-FLAIR and T1-weighted images were evaluated by a clinical neuroradiologist for abnormalities. Second, grey matter (GM) morphometrical features were analysed using Mindboggle (v1.3.8) ${ }^{106}$. Total brain, GM and white matter (WM) volumes, as well as the volumes of each subcortical structure were estimated. Mindboggle was used to estimate mean thickness, surface area, mean travel depth and mean curvature for each cortical region. Third, WM microstructure was investigated with a fixel-based analysis on the diffusion-weighted images, resolving crossing fibre populations within a voxel, using MRtrix ${ }^{205}$ (v3.0). This analysis gives a within-fixel measure of fibre density (FD), a macroscopic Jacobian-based measure of fibre cross-section (FCS) and a combined measure termed Fibre Density and Cross-section (FDC) ${ }^{125}$. Next, functional connectivity was estimated based on bivariate correlations in the rs-fMRI signal between brain regions. In order to limit the number of statistical comparisons, only regions of the 
default mode (DMN), fronto-parietal (FPN), dorsal attention (DAN) and salience networks (SN) were selected from the CONN toolbox $(\mathrm{v} 19 . \mathrm{b})^{206}$ network atlas, based on the a priori hypothesis of potential impact of chemotherapy on attention and executive functioning. This resulted in a total of 17 regions included in this analysis. Finally, whole-brain structural and functional organization, using normalized weighted graphs respectively based on whole-brain constrained spherical deconvolution (CSD) tractography ${ }^{126}$ and bivariate correlations between the rs-fMRI timeseries, were investigated using graph theory measures of characteristic path length, global and local efficiency and clustering coefficient ${ }^{207}$.

\subsubsection{Statistical analysis}

Demographic and psycho-behavioral parameters were compared between study and control groups (SPSS v.19.0), using Mann-Whitney U-tests.

For each imaging outcome parameter two group analyses were conducted: 1) All children born after cancer-complicated pregnancies (the whole study group) versus matched controls and 2) the subgroup of children with prenatal chemotherapy exposure versus matched controls. Based on previous observations of the impact of prematurity on the neurocognitive outcome in children born after cancer-complicated pregnancies ${ }^{5-7 ; 10}$, a general linear model (GLM) with group, normalized GA and the group-by-GA interaction was used. When no significant main effect of GA or group-by-GA interaction was observed, this model was simplified to only include group as a predictor. Intracranial volume (ICV) was added as a covariate in the analysis of FCS, FDC ${ }^{208}$ and measures of volume. False-discovery-rate (FDR) correction was used for multiple comparisons in ROI-to-ROI functional connectivity and grey matter structure, whereas family-wise-error (FWE) correction in combination with connectivity-based fixel enhancement (CFE) ${ }^{209}$ was used for the fixel-based analyses, as further detailed in the supplementary materials. Significance was assessed at $p<.05$.

Mean values of parameters in regions with a significant group effect were extracted for each participant. Exploratory post-hoc analyses were performed with SPSS investigating the association between these regional parameters of brain functioning/structure with psycho-behavioural functioning and obstetrical/oncological outcomes, using Spearman correlations for numerical variables and Mann-Whitney U-test for categorical variables. The following obstetrical and oncological variables were used for post-hoc analyses in the whole study group: mother deceased (yes/no), chemotherapy during pregnancy (yes/no), radiotherapy during pregnancy (yes/no), surgery during pregnancy (yes/no), and customized birth weight percentile ${ }^{201}$. Within the group with chemotherapy during pregnancy, the following clinical variables were additionally tested: anthracyclines (yes/no), platinum derivatives (yes/no), 5-FU (yes/no), cyclophosphamide 
(yes/no), GA at start chemotherapy and duration of chemotherapy (adjusted for chemo regimen) during pregnancy.

\subsubsection{Role of funding}

The funding sources had no role in writing of the manuscript or the decision to submit it for publication.

\subsection{Results}

\subsubsection{Sample size and demographics}

In total, 84 children (42 in each group) were included in the final analysis, of whom 6 (3 in each group) were excluded for the morphometrical analysis (Figure 6.1). From the initial dataset, 4 children, of whom two were prenatally exposed to chemotherapy and the other two being control children, were excluded due to incidental neurological findings, detailed in the supplementary materials. For none of the cases, a direct link could be made to the maternal cancer history or treatment. In both study and control group, 42 children were included in the final analysis, of whom 3 were excluded for the morphometrical analysis. From the initial dataset, 4 children, of whom two were prenatally exposed to chemotherapy and the other two being control children, were excluded due to incidental neurological findings, detailed in the supplementary materials. For none of the cases, a direct link could be made to the maternal cancer history or treatment.

Population characteristics, clinical history and neuropsychological outcomes are shown in Tables 6.1 to 6.3 . Thirty mothers in the study group (71\%) received chemotherapy during pregnancy, of which five (16\%) additionally received radiotherapy and $24(80 \%)$ underwent surgery. Chemotherapy during pregnancy varied in regimen (see Table 6.2)), GA at start therapy (Median $=20$ weeks 4 days, interquartile range $=18$ weeks 2 days to 25 weeks 5 days, minimum $=14$ weeks) and duration (Median $=12$ weeks 2 days, interquartile range $=9$ weeks 7 days to 16 weeks 1 day). One mother received Trastuzumab only during pregnancy. Breast cancer was the most often diagnosed cancer $(n=25,60 \%)$, followed by haematological $(n=5$, $12 \%)$ and gynaecological $(n=4,10 \%)$ cancers. Within the study group, intelligence testing was performed using WISC-III in 30 children and using WISC-V in 11 children.

52 children (62\%), 26 in each group, were born preterm (before 37 weeks GA), of which the majority was born late preterm $(G A=32-37$ weeks, study group $n=23$, control group $n=22)$. In 


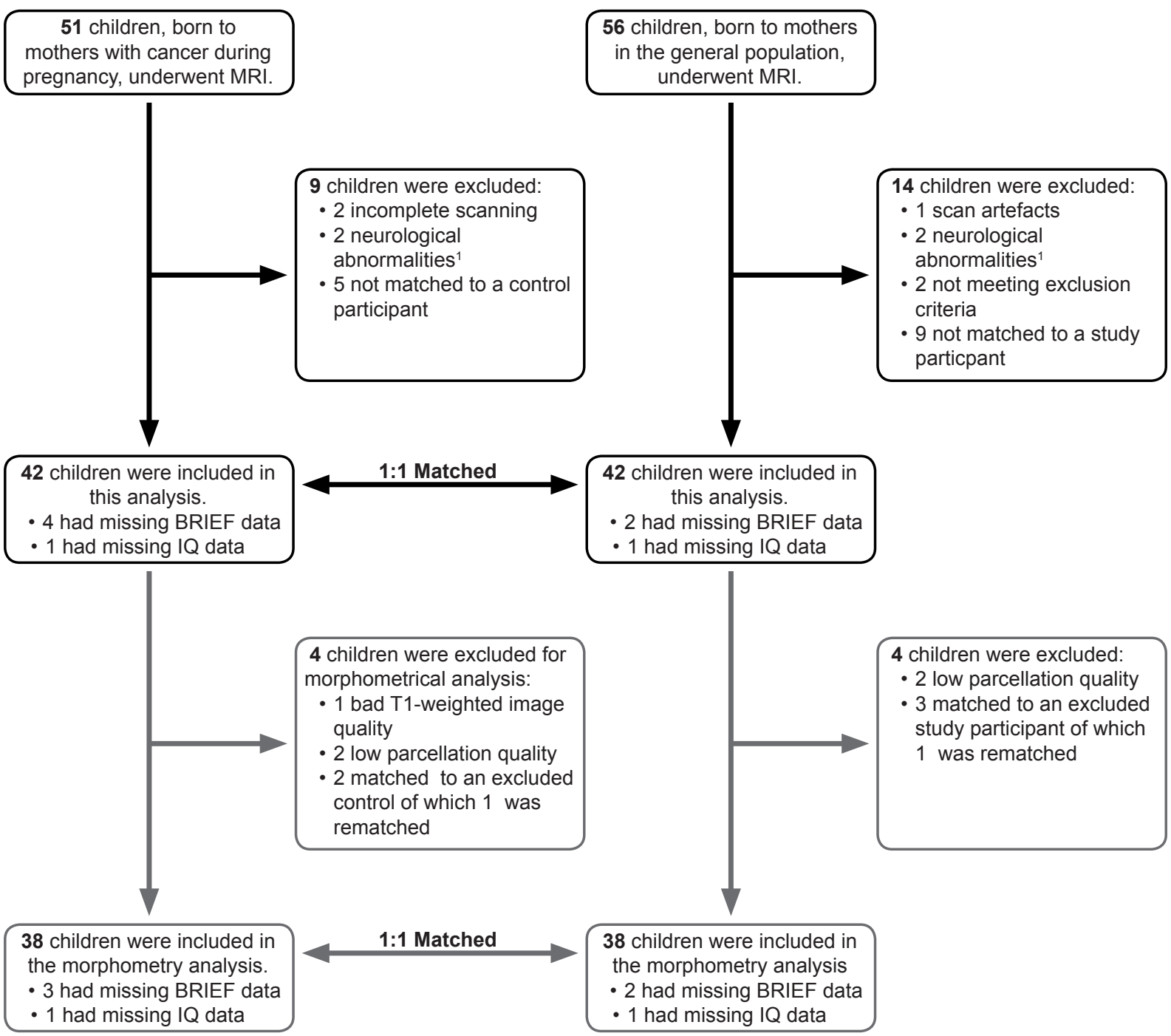

Figure 6.1 - Flow diagram of recruitment for this study. Of the 51 study and 56 control participants who were scanned, 42 of each group were included in the final analysis. See supplementary materials for an overview of observed neurological abnormalities 
Table 6.1 - Population characteristics. Prematurity classification is reported in accordance to the WHO classification: Very preterm children have GA 28-32 weeks, moderate to late preterm children have GA 32-37 weeks. Ethnicity was determined by the self-reported ethnicity of both parents. Between group differences were assessed using using Mann-Whitney U-test. *Ethnicity was compared as Caucasian vs. non-caucasian. Q1: first quartile. Q3: third quartile. Min: minimum. Max: maximum GA: gestational age at birth. LGA: large for gestational age, defined as above the $90^{\text {th }}$ percentile. SGA: small for gestational age, defined as below the 10 th percentile.

\begin{tabular}{|c|c|c|c|c|c|c|c|}
\hline & \multicolumn{3}{|c|}{ Study group $(n=42)$} & \multicolumn{3}{|c|}{ Control group $(n=42)$} & \multirow[b]{2}{*}{ p-value] } \\
\hline & Median & Q1 & Q3 & Median & Q1 & Q3 & \\
\hline Age (years) & 9.19 & 9.07 & 9.27 & 9.34 & 9.12 & 9.67 & .008 \\
\hline GA (weeks+days) & $36+3$ & $34+4$ & $37+7$ & $36+1$ & $34+3$ & $38+0$ & .941 \\
\hline Birth weight (g) & 2648 & 2099 & 3013 & 2930 & 2268 & 3213 & .365 \\
\hline \multirow[t]{2}{*}{ Maternal age at birth (years) } & 32 & 30 & 35 & 31 & 29 & 33 & .036 \\
\hline & Count & $\%$ & & Count & $\%$ & & \\
\hline Prematurity & & & & & & & .958 \\
\hline Very preterm & 3 & $7 \%$ & & 2 & $5 \%$ & & \\
\hline Moderate to late preterm & 23 & $55 \%$ & & 24 & $57 \%$ & & \\
\hline Full term & 16 & $38 \%$ & & 16 & $38 \%$ & & \\
\hline \multicolumn{8}{|l|}{ Birth weight percentile } \\
\hline SGA & 7 & $17 \%$ & & & & & \\
\hline LGA & 2 & $5 \%$ & & & & & \\
\hline Twins & 6 & $14 \%$ & & 1 & $2 \%$ & & .109 \\
\hline Sex (n male) & 21 & $50 \%$ & & 21 & $50 \%$ & & 1 \\
\hline Ethnicity & & & & & & & $*_{1}$ \\
\hline Caucasian & 38 & $90 \%$ & & 37 & $88 \%$ & & \\
\hline African & 4 & $10 \%$ & & & & & \\
\hline Mixed & & & & 5 & $12 \%$ & & \\
\hline Level of education parents & & & & & & & .541 \\
\hline Mother & & & & & & & \\
\hline Primary school & 2 & $5 \%$ & & & & & \\
\hline Secondary school & 11 & $26 \%$ & & 6 & $14 \%$ & & \\
\hline Bachelor & 12 & $29 \%$ & & 21 & $50 \%$ & & \\
\hline Master & 17 & $40 \%$ & & 13 & $31 \%$ & & \\
\hline Father & & & & & & & .605 \\
\hline Primary school & 2 & $5 \%$ & & & & & \\
\hline Secondary school & 16 & $38 \%$ & & 15 & $36 \%$ & & \\
\hline Bachelor & 9 & $21 \%$ & & 12 & $29 \%$ & & \\
\hline Master & 15 & $36 \%$ & & 14 & $33 \%$ & & \\
\hline Smoking during pregnancy & 3 & $7 \%$ & & 2 & $5 \%$ & & .676 \\
\hline Drugs during pregnancy & 0 & $0 \%$ & & 0 & $0 \%$ & & 1 \\
\hline Alcohol during pregnancy & 3 & $7 \%$ & & 5 & $12 \%$ & & .712 \\
\hline Maternal death & 6 & $14 \%$ & & 0 & $0 \%$ & & .026 \\
\hline
\end{tabular}


the study group, $17 \%$ of children were born small for gestational age (SGA, defined as below 10 th customized birth weight percentile). Unfortunately, six children $(14 \%)$ in the study group had lost their mother by the time of assessment, whereas all mothers in the control group were alive. Mann-Whitney U-test revealed a small but significant difference in age between both groups (study group: median $=9.19$ years, interquartile range $=9.07-9.27$ years, control group: median $=9.34$ years, interquartile range $=9.12-9.67$ years, $p=.008)$. Mothers in the study group were on average older at birth compared to the control group (study group: median $=32$ years, interquartile range $=30-35$ years, control group: median $=31$ years, interquartile range $=29-33$ years, $\mathrm{p}=.036$ ). For both groups, all psycho-behavioral measures were within normal ranges, though children in the study group showed a slightly lower total IQ (median=106, interquartile range $=96-114, p=.028)$, compared to controls $($ median $=109$, interquartile range $=102-119)$. 
Table 6.2 - Maternal disease and treatment during pregnancy. 5-FU: 5-Fluorouracil. FEC: 5-fluorouracil, epirubicin and cyclophosphamide. FAC: 5-fluorouracil, doxorubicin (adiamycin) and cyclophosphhamide. AC: doxorubicin and cyclophosphamide. EC: epirubicin and cyclophosphamide. ABVD: Doxorubicin, Bleomycin, Vinblastine, Dacarbazine.

\begin{tabular}{|c|c|c|}
\hline Maternal disease $(n=42)$ & Count & $\%$ \\
\hline Breast cancer & 25 & $60 \%$ \\
\hline Cervical cancer & 3 & $7 \%$ \\
\hline Ovarian cancer & 1 & $2 \%$ \\
\hline Hodgkin lymphoma & 3 & $7 \%$ \\
\hline Tongue cancer & 3 & $7 \%$ \\
\hline Leukaemia & 2 & $5 \%$ \\
\hline Brain Tumour & 2 & $5 \%$ \\
\hline Melanoma & 1 & $2 \%$ \\
\hline Kidney carcinoma & 1 & $2 \%$ \\
\hline Colon carcinoma & 1 & $2 \%$ \\
\hline \multicolumn{3}{|l|}{ Maternal treatment during pregnancy $(n=42)$} \\
\hline Chemotherapy & 30 & $71 \%$ \\
\hline Targeted therapy & 1 & $2 \%$ \\
\hline Radiotherapy & 5 & $12 \%$ \\
\hline Surgery & 29 & $69 \%$ \\
\hline No treatment & 6 & $14 \%$ \\
\hline \multicolumn{3}{|l|}{ Chemotherapy regimen $(n=30)$} \\
\hline $\mathrm{FEC} / \mathrm{FAC}$ & 11 & $37 \%$ \\
\hline $\mathrm{AC} / \mathrm{EC}$ & 7 & $23 \%$ \\
\hline ABVD & 3 & $10 \%$ \\
\hline Cisplatin & 3 & $10 \%$ \\
\hline Carboplatin and 5-FU & 2 & $7 \%$ \\
\hline $5-F U$ & 1 & $3 \%$ \\
\hline Daunorubicin/Cytarabine & 1 & $3 \%$ \\
\hline Epirubicin & 1 & $3 \%$ \\
\hline Temozolomide & 1 & $3 \%$ \\
\hline
\end{tabular}


Table 6.3 - Neuropsychological outcomes of the child Between group differences were assessed using using Mann-Whitney U-test WISC: Wechsler Intelligence Scale for Children (version III or V). CBCL: Child Behavior Checklist. BRIEF: Behavior Rating Inventory of Executive Function. $\mathrm{n}_{\mathrm{s}}$ : number of children in the study group. $\mathrm{n}_{\mathrm{c}}$ : number of children in the control group. Q1: first quartile. Q3: third quartile

\begin{tabular}{|r|ccccccc|c|}
\cline { 2 - 9 } \multicolumn{1}{c|}{} & \multicolumn{7}{c|}{ Study group $(\mathbf{n = 4 2})$} & \multicolumn{3}{c|}{ Control group $(\mathbf{n = 4 2})$} & \\
& Median & Q1 & Q3 & Median & Q1 & Q3 & $p$-value \\
\hline Full scale IQ & 106 & 96 & 114 & 109 & 102 & 119 & .028 \\
Verbal IQ (WISC-III, $\left.\mathrm{n}_{\mathbf{s}}=30, \mathrm{n}_{\mathrm{c}}=29\right)$ & 108 & 95 & 114 & 111 & 103 & 117 & .285 \\
CBCL (T-scores) & & & & & & & \\
Internalizing problems & 53 & 45 & 61 & 48 & 43 & 58 & .303 \\
Externalizing problems & 49 & 41 & 54 & 44 & 40 & 51 & .170 \\
Total problems & 52 & 44 & 61 & 47 & 43 & 53 & .111 \\
BRIEF (T-scores) & & & & & & & \\
Behavioral regulation & 50 & 43 & 60 & 45 & 42 & 54 & .357 \\
Metacognition & 52 & 45 & 60 & 52 & 47 & 58 & .700 \\
Global executive composite score & 51 & 43 & 61 & 51 & 44 & 57 & .451 \\
\hline
\end{tabular}

\subsubsection{Imaging analysis}

No significant differences were found in total brain $(p=.14)$, WM $(p=.30)$ or $\operatorname{GM}(p=.09)$ volume (Table 8.5).Significantly lower cortical travel depth in the left superior temporal cortex (Table 6.4), indicating less pronounced gyrification, was observed in both the study group $\left(p_{F D R}=.002, F(1,74)=19.75\right)$ and the chemotherapy subgroup $\left(p_{F D R}=.006, F(1,53)=\right.$ 17.9), compared to matched controls. In both analyses, GA at birth was associated negatively with mean curvature, indicating a more outward-curving surface, in the left posterior cingulate $\operatorname{cortex}\left(\right.$ resp. $p_{F D R}=.03, F(1,72)=13.16$ and $\left.p_{F D R}=.03, F(1,50)=12.6\right)$.

Table 6.4 - Cortical morphometric measures. Only measures with a significant effect of group, GA or group by GA are shown. Significance is assessed at $p<.05$, FDR (Benjamini-Hochberg) corrected for assessing 62 regions.

\begin{tabular}{|c|c|c|c|c|c|c|c|c|c|}
\hline \multirow[t]{2}{*}{ Study group } & \multirow[t]{2}{*}{ Parameter } & \multirow[t]{2}{*}{ Region } & \multicolumn{2}{|c|}{ Study group } & \multicolumn{2}{|c|}{ Control group } & \multicolumn{3}{|c|}{$P_{\mathrm{FDR}}$} \\
\hline & & & Mean & SD & Mean & SD & Group & $\mathrm{GA}$ & $\begin{array}{l}\text { Group } \\
\text { by GA }\end{array}$ \\
\hline All cancers $(n=38)$ & Travel depth & Left superior temporal & 8.25 & 0.60 & 8.83 & 0.54 & .002 & .535 & .966 \\
\hline All cancers $(n=38)$ & Mean curvature & Left posterior cingulate & -3.41 & 0.25 & -3.48 & 0.23 & .72 & .033 & .857 \\
\hline Chemo $(n=27)$ & Travel depth & Left superior temporal & 8.21 & 0.63 & 8.86 & 0.50 & .006 & .535 & .966 \\
\hline Chemo $(n=27)$ & Mean curvature & Left posterior cingulate & -3.42 & 0.26 & -3.47 & 0.26 & .99 & .032 & .857 \\
\hline
\end{tabular}

Concerning WM microstructure, a significantly lower FDC (Figure 6.2:A ,7-17\% mean difference, 465 fixels) was observed in study group children compared to controls bilaterally in the forceps major of the corpus callosum (CC) including its occipital tapetal fibres and a higher FCS (Figure 6.2:B ,4-5\% mean difference, 8 fixels) in the subcortical white matter of the left 
dorsolateral prefrontal cortex region. The latter fixels most likely belong to the anterior thalamic radiation, though the inferior fronto-occipital fasciculus or uncinate fasciculus are also possibilities as all these tracts traverse this region. Compared to controls, children with prenatal chemotherapy exposure showed a lower FCS (Figure 6.2:C,6-10\% mean difference, 112 fixels) in the right tapetal fibres towards the post-central sulcus, a lower FDC (Figure 6.2:D ,11-22\% mean difference, 29 fixels) in the right tapetal fibres in the centrum semiovale deep to the post-central gyrus and a small group of fixels in the right side of the splenium of the CC, and a higher FDC (Figure 6.2:E ,13-23\% mean difference, 11 fixels) in the right anterior thalamic radiation. No significant effects in any fixel-based measures of GA or its interaction with maternal cancer or chemotherapy during pregnancy were found. Abovementioned findings are visualized at a trend level $\left(p_{F W E}<.1\right)$ in Figure 8.1.

No significant effects of cancer/chemotherapy during pregnancy, GA or their interaction in ROI-to-ROI functional coherence were observed after correction for multiple comparisons. However, when applying a more liberal multiple comparison correction for the ROI-to-ROI functional connectivity, accounting for the 17 regions (instead of the total number of connections) using a family-wise error correction, a significantly lower connectivity between the right lateral pole and intraparietal sulcus is observed in both the whole study group $\left(p_{F W E, R O I}=.026, T=-3.32\right)$ and the chemotherapy subgroup $\left(p_{F W E, R O I}=.050, T=-3.15\right)$, compared to controls. Finally, no significant effects were observed in the structural or functional whole-brain graph measures. 


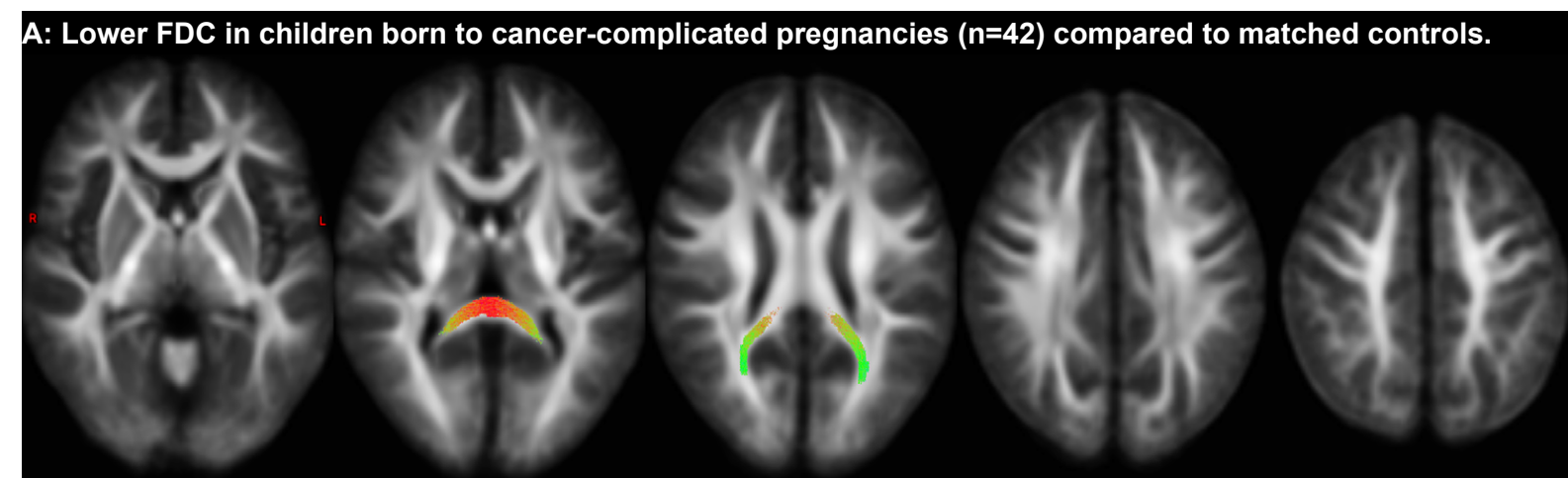

B: Higher FCS in children born to cancer-complicated pregnancies ( $n=42)$ compared to matched controls.

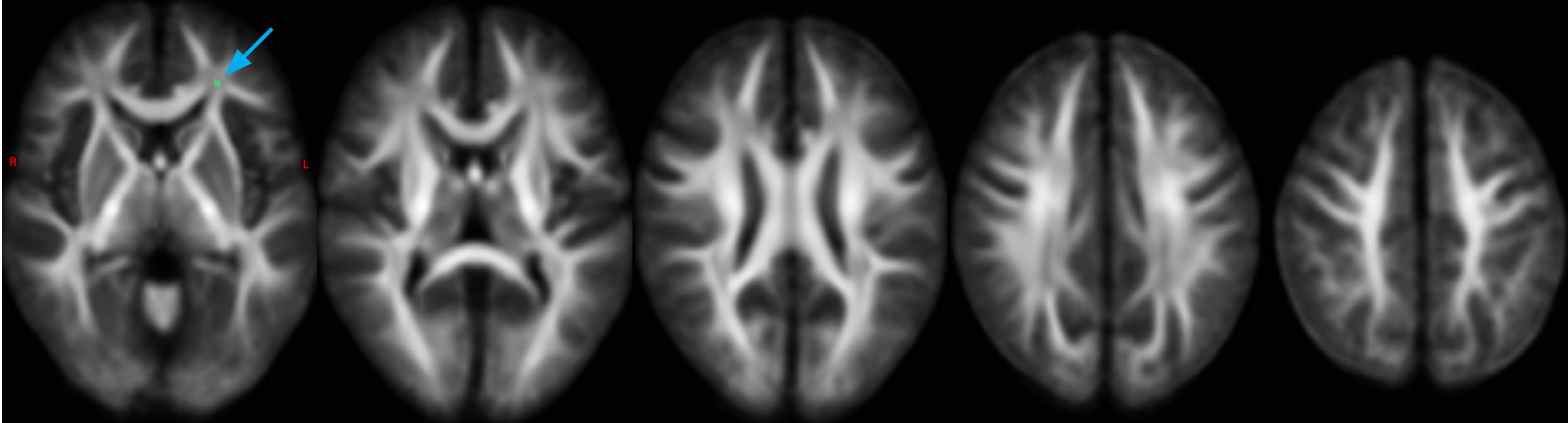

C: Lower FCS in children with prenatal chemotherapy exposure $(n=30)$ compared to matched controls.

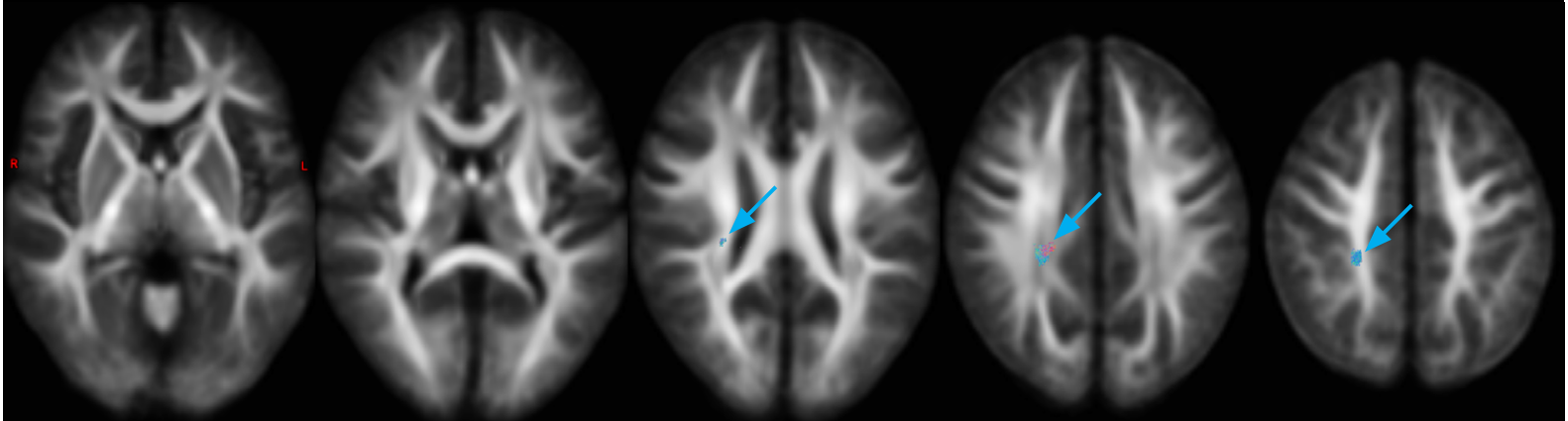

D: Lower FDC in children with prenatal chemotherapy exposure $(n=30)$ compared to matched controls.

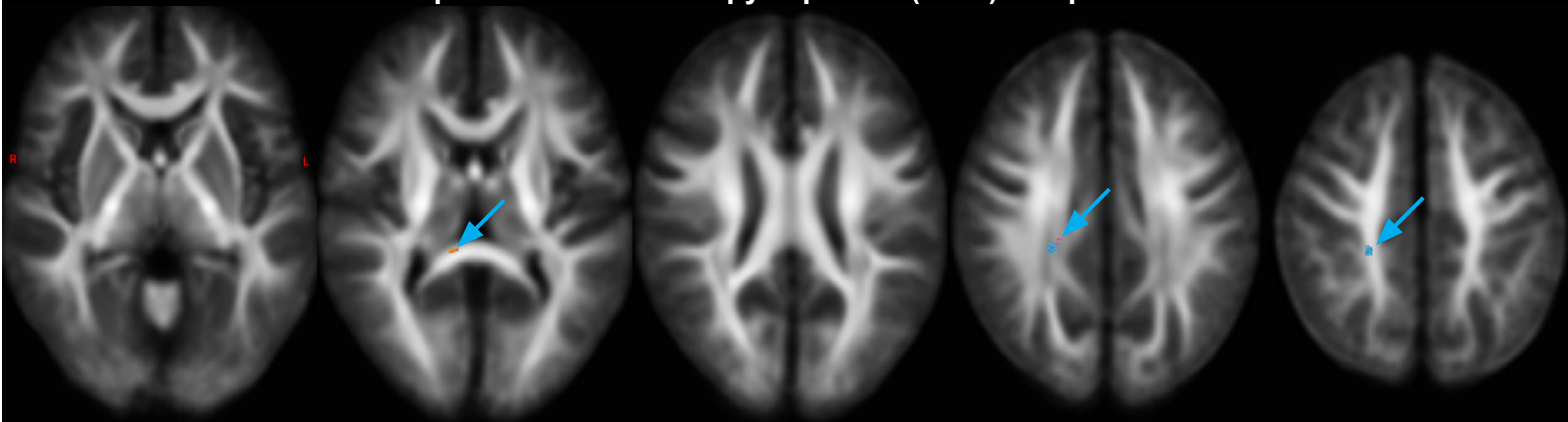

E: Higher FDC in children with prenatal chemotherapy exposure $(n=30)$ compared to matched controls.

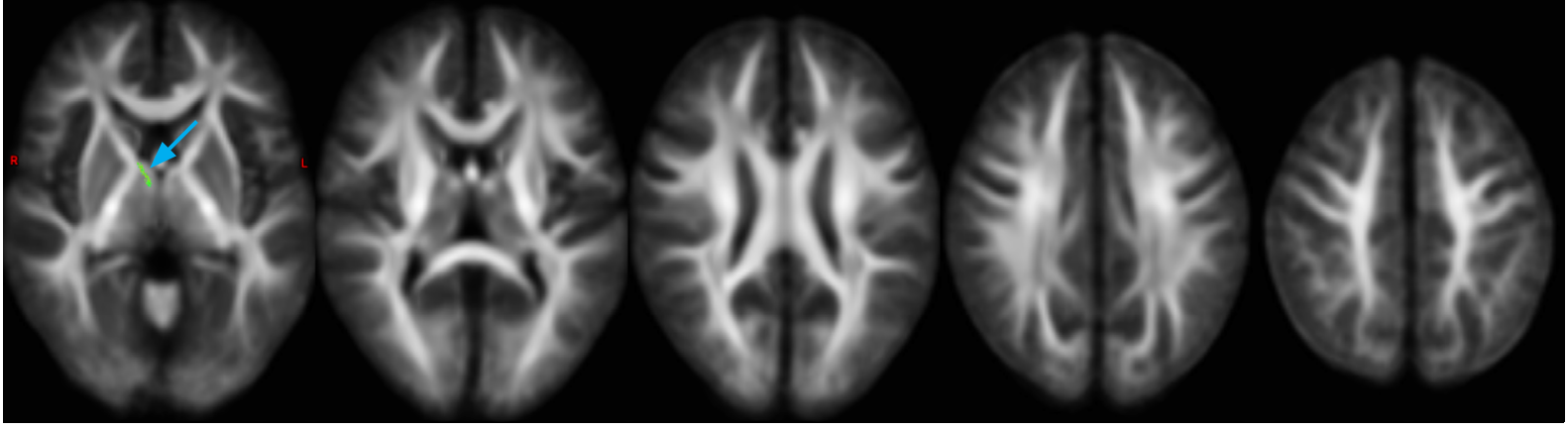

Figure 6.2 - Observed differences in WM microstructure. Significance is assessed at $p<.05$, using connectivity-based fixel enhancement ${ }^{209}$ with family-wise-error correction. All results are rendered with 200000 streamlines (as described earlier $\left.{ }^{125}\right)$ ), mapped on $10 \mathrm{~mm}$ interval slices and coloured with conventional directional colour encoding. FCS: fibre cross-section. FDC: Fibre density and cross-section 


\subsubsection{Relationship to maternal clinical history and psychological out- come}

None of the psycho-behavioural or maternal clinical parameters were significantly related to any of the brain differences in the study group. While chemotherapy was never indicated as a significant risk factor, it did always trend towards more extensive impact. Within the subgroup of children with prenatal chemotherapy exposure, Mann-Whitney U-test revealed significantly lower travel depth in the left superior temporal cortex in children exposed to platinum derived chemotherapy during pregnancy compared to other chemotherapies ( $p=.028, \mathrm{U}=20$, Figure $6.3 \mathrm{a})$. In the regions where we observed significantly lower FDC in prenatally chemotherapy exposed children compared to matched controls (Figure 6.2:D), the FDC was less reduced for anthracyclines, compared to other chemotherapeutic agents $(p=.048, \mathrm{U}=40$, Figure $6.3 \mathrm{~b})$. 


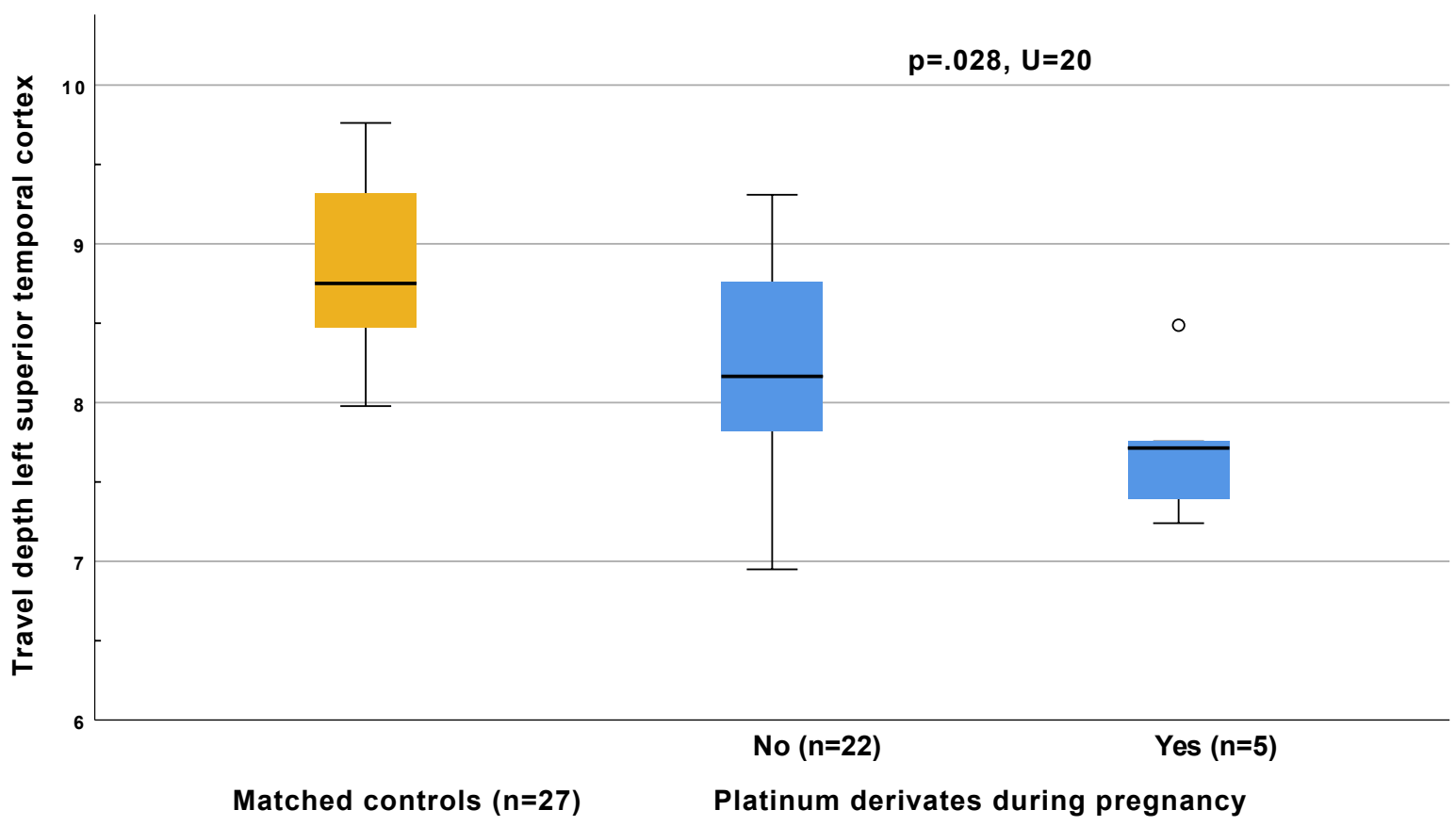

(a) Travel depth in the superior temporal cortex differs between platinum derivatives during pregnancy compared to other chemotherapeutic agents.

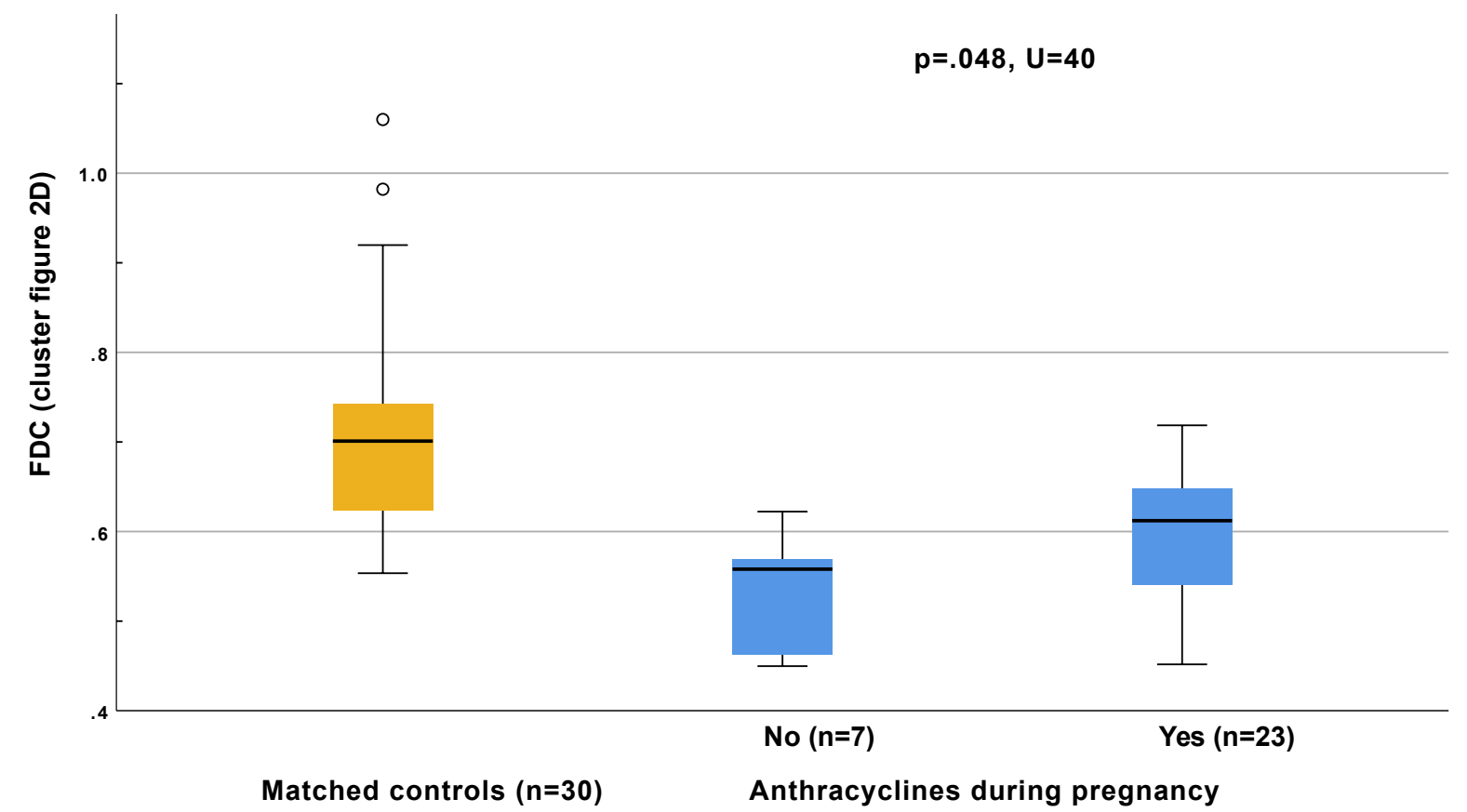

(b) Fibre density and cross-section differs (Figure 6.2:D) between anthracyclines during pregnancy compared to other chemotherapeutic agents.

Figure 6.3 - Relationship of brain differences with maternal clinical history. MW U-test was used to assess significant relations $(p<.05)$ 


\subsection{Discussion}

To our knowledge, this is the first study applying multimodal MRI to assess brain structure and function in children born of cancer-complicated pregnancies. We found macro- and microstructural differences within the WM and GM between children born to women with cancer compared to matched controls, but no significant differences in functional connectivity or whole-brain organization were observed. Furthermore, no correlations between local structural brain differences and psychological outcome were noted. This indicates that these local structural effects have a limited effect on global and functional organization of the brain.

The differences observed in this study cannot be solely ascribed to the effect of chemotherapy, but rather to a combination of secondary mechanisms. Indeed, although differences in WM microstructure resemble results in literature of cancer survivors receiving intravenous chemotherapy ${ }^{27 ; 29}$, chemotherapy during pregnancy was not identified as a significant risk factor in any of the post-hoc analyses. The psychosocial impact of a cancer diagnosis during pregnancy might partially explain these findings. A recent study identified maternal death as a potential risk factor in their findings of decreased verbal intelligence in children born after cancer-complicated pregnancies ${ }^{14}$ and another study described a relationship between infant behavioural functioning and maternal psychological wellbeing ${ }^{82}$. However, given that only $12 / 42$ mothers in the study group did not receive chemotherapy during pregnancy and the heterogeneity of chemotherapy regimens used, we cannot exclude that some chemotherapeutics might directly or indirectly affect neurodevelopment.

In the whole study group, as well as in the subgroup of prenatally chemotherapy-exposed children, compared to their respective matched controls, we found a lower cortical travel depth in the left superior temporal gyrus, indicating an impact on the gyrification of the cortex. Gyrification of the brain has been found to peak around 30 weeks of gestation ${ }^{210}$, with the left superior temporal gyrus developing slightly later than its right counterpart ${ }^{211}$. The left superior temporal cortex has an established role in the language network ${ }^{212}$, which might link current findings of decreased gyrification in this region to earlier findings of lower verbal intelligence ${ }^{14}$. However, a direct link between travel depth in this region and verbal intelligence was not observed here.

Post-hoc exploratory analyses indicated platinum derivatives as a potential risk factor for this decreased gyrification. Platinum derivatives have a high transplacental transfer ${ }^{9 ; 1 ; 213}$ and have earlier been associated with a higher risk for SGA ${ }^{2}$. Moreover, 3/5 platinum cases received cisplatin which has been associated with potential hearing loss ${ }^{14 ; 214-216}$, potentially impacting language development. However, as no data on hearing functionality was available in this cohort, a direct link could not be established. 
Regarding the WM, we observed a lower FDC in the splenium of the CC and its tapetal fibres in the whole study group and a significantly lower FDC and FCS in the chemotherapy subgroup in the same region, suggesting a regional thinner axonal bundle diameter and lower axonal count. Similarly to this study, diffusion-weighted imaging has indicated impaired WM microstructure in the posterior part of the CC in survivors of paediatric soft tissue and bone sarcomas ${ }^{27}$, as well as in adult breast cancer survivors ${ }^{29}$. The dense packing and high vascularity of the CC might partially explain its particular vulnerability to neuro-inflammation and demyelination ${ }^{217}$, potentially induced by chemotherapy exposure.

Within the chemotherapy subgroup, the lower FDC was less pronounced when mothers received anthracyclines, indicating that these anthracycline-based combination treatments might generally provide a lower risk for affecting the developing brain.

Interestingly, in the whole study group a higher FCS was observed in the left prefrontal subcortical WM, with similarly a higher FDC being observed in the chemotherapy subgroup in the right anterior thalamic radiation. Both findings indicate stronger structural connectivity in the anterior thalamic radiations compared to controls. Seeing the importance of this WM tract for executive functioning ${ }^{61}$, which was found earlier to be affected in both this population ${ }^{196}$ and cancer survivors $^{21 ; 23 ; 27}$, the increase in FDC/FCS in this structure might result from a neuroplastic compensatory mechanism. However, this might also reflect altered WM developmental patterns due to changes in the pre- and postnatal environment, both directly and indirectly linked to the maternal cancer.

Prematurity in this study only showed a significant impact on the mean curvature in the left posterior cingulate, resonating with earlier findings of altered functional connectivity with prematurity in this region ${ }^{70}$, as well as with earlier findings on the discrepancy between in utero and postnatal gyrification ${ }^{218}$. On the other hand, the observed limited effect of GA confirms earlier findings that neurocognitive effects of prenatal exposure to cancer and its therapy are not limited to the frequent occurrence of preterm birth ${ }^{14 ; 196}$.

Some limitations of this study should be mentioned. Due to the rare incidence of cancer during pregnancy, data acquisition spanned a five-year period. The effects of scanner variability during this period were limited by keeping the scanner set-up and protocol unchanged over the whole period and by simultaneously recruiting study and control group children. Next, while the total sample size allows for observations on overall group effects, the heterogeneity of this population necessitates caution in the interpretation of the results for specific therapies during pregnancy. Furthermore, only 12/42 mothers in the study group did not receive chemotherapy during pregnancy, and this group of patients is often diagnosed later in pregnancy or with less aggressive 
tumours, making it especially difficult to distinguish effects of therapy from other cancer-related mechanisms. Moreover, when interpreting these findings, we should note that this study only included Belgian patients, treated between 2005 and 2010. Indeed, this study cannot account for international differences in treatment and more recent advances in clinical more. On the other hand, most treatments women received in this cohort still adhere to today's guidelines ${ }^{12 ; 219-221}$. Furthermore, non-cancer-related heterogeneity was limited by testing children within a small age range and effects of prematurity were controlled for by matching controls on GA on a 1:1 ratio. Finally, no data on maternal stress and anxiety were available for these children, making it difficult to discriminate between prenatal stress-related or cancer-related impact on neurodevelopment. However, current findings do not align with previous findings on the effects of prenatal stress on neurodevelopment ${ }^{63 ; 128}$, making it an unlikely pathway to completely explain the discrepancies in brain development observed in this study.

\subsection{Conclusions}

This study observed local structural WM and GM differences, but no whole-brain or functional differences, in children born after cancer-complicated pregnancies compared to matched controls. Platinum derivatives during pregnancy were indicated as a potential risk factor for decreased cortical gyrification of the left superior temporal gyrus. However, chemotherapy during pregnancy in general did not significantly contribute to these findings, resonating with earlier findings ${ }^{14 ; 82}$ describing the role of the psychosocial impact of cancer during pregnancy on the neurocognitive functioning of the child. Balancing between pros and cons on the use of chemotherapy during pregnancy, the current data favour the use of chemotherapy during pregnancy when clinically indicated. 


\title{
CHAPTER
}

\author{
7
}

DISCUSSION

This thesis investigated the effect of maternal cancer and its treatment during pregnancy on neurodevelopment of the offspring at the age of nine. First, in chapter 4 we investigated the effect of chemotherapy on brain volume in a longitudinal cohort of young and older non-pregnant patients with breast cancer, observing a widespread, age-dependent WM volume expansion, suggesting chemotherapy-induced neuroinflammation. Next, in chapter 5 we employed ERPs to investigate the effect of chemotherapy during pregnancy on the child's development of executive functions. We found prenatal cancer and chemotherapy exposure to affect the development of response inhibition and spatial attention. Finally, in chapter 6 we applied multimodal MRI to investigate structural and functional brain development in children born after cancer-complicated pregnancies, compared to matched controls. Here, we observed local differences in GM and WM structure, but not in brain organization or functional neurodevelopment. While platinum-based chemotherapy was indicated as a risk factor for these GM differences, this was not the case for chemotherapy in general.

The following sections will interprete findings from the different studies in chapters 4 to 6 within the wider literature and the clinical context of cancer in pregnancy. First, we will provide insights on the neurocognitive impact of chemotherapy in different cancer survivor populations. Next, we will discuss the relevance and impact of our findings within the context of cancer in pregnancy. Then, some of the limitations of the current study will be discussed. Based on current findings and their limitations, we will give suggestions for future research. Finally, we will provide the 
main conclusions of this dissertation.

\subsection{Cancer, chemotherapy and the brain}

Similar to the results of this work, multiple studies in cancer survivors have described the impact of chemotherapy on executive functioning ${ }^{18 ; 21 ; 23 ; 24}$ and white $27 ; 27-31$ and grey matter structure $24 ; 32-36$, in both pediatric and adult cancer survivors. Moreover, the findings in chapter 4 , where we observed age-dependent WM volume expansion in adult breast cancer patients after chemotherapy, which were largely in line with the results of an independent mouse study ${ }^{166}$, strengthened the hypothesis of chemotherapy-induced neuroinflammation, which might damage both GM and WM. The corpus callosum in particular, which was found to be altered in structure in this work (chapter 6) after prenatal exposure to maternal cancer, as well as both in studies with adult breast cancer ${ }^{29}$ and childhood sarcoma ${ }^{27}$ survivors, might be especially vulnerable to such impact, as it is highly vascularized and has a dense axonal packing ${ }^{217}$.

While the observed neurocognitive decline after non-cns cancer treatment is often subclinical, this is not always the case. For instance, in a recent review, we found that leuko-encephalopathy occurs in $17-87 \%$ of patients with acute lymphoblastic leukaemia, which was associated with the methothrexate dose, and up to $50 \%$ of solid tumour patients ${ }^{26}$. However, this effect is not as well documented in other non-cns cancer populations.

Moreover, the neurocognitive impact of chemotherapy can be long-lasting, having an impact on the quality of life ${ }^{21 ; 38}$. For instance, we observed functional and structural GM differences, in addition to earlier findings of impacted quality of life, working memory, processing speed, and visual memory, higher risk for leukoencephalopathy and differences in WM microstructure ${ }^{25 ; 27}$, in childhood sarcoma survivors, compared to healthy controls, 2-20 years after the end of therapy ${ }^{36}$.

On the other hand, recent studies indicated potential, although partial, recovery of this chemotherapy-induced neurocognitive impact ${ }^{20 ; 30}$. To further mediate this recovery, we could employ a pharmacological or rehabilitation treatment. For instance, in a recent pilot study, we showed that a mindfulness-based therapy might increase functional connectivity in the attention networks and alleviate cognitive complaints, fatigue and emotional distress ${ }^{222}$.

In chapter 4, when comparing young and older patients with breast cancer, we observed psychological outcome and changes in brain volume after therapy to be age-dependent. Multiple physiological changes with age and potentially co-occurring frailty might make the aged brain especially vulnerable to chemotherapy-induced neurotoxicity. Similarly, chemotherapy might 
interact differently with different developmental processes. For instance, in a study observing the structural and functional impact of cancer treatment in childhood sarcoma survivors, we found younger age at diagnosis to be related to lower right lateral orbitofrontal cortical thickness ${ }^{36}$. The results of this study indicate that chemotherapy interacts with processes of cortical pruning during adolescence, with particular vulnerability of frontal and limbic regions. Moreover, in this same population, Sleurs et al. ${ }^{25}$ found younger patients to be more vulnerable to leukoencephalopathy induced by alkylating agents, whereas older children were more prone to methothrexate-induced leukoencephalopathy. However, when investigating the neurocognitive impact of maternal cancer treatment in chapter 6 , we did not observe a link between gestational age at maternal treatment and brain development. While such link, apart from the effects of therapy during the first trimester, might be absent, current study might have been statistically underpowered. During pregnancy, the brain goes through a multitude of overlapping stages of development, which when affected might result in distinct pathologies ${ }^{16}$. On the other hand, the fetal and infant brain has a high neuroplastic capacity, which might partially compensate the impact of chemotherapy exposure on brain development ${ }^{58-60}$

\subsection{Neurodevelopment after prenatal exposure to cancer treatment}

In contrast to neuroimaging findings from cancer survivor studies, we did not find significant differences in functional connectivity or brain organization between children born to cancercomplicated pregnancies and matched controls. Moreover, whereas cancer survivor studies describe widespread WM and GM changes, the findings in chapter 6 describe more localized effects. Furthermore, these structural differences were not found to significantly differ when chemotherapy was administered during pregnancy.

Previous studies have associated frequent occurrence of preterm births after cancer in pregnancy to be associated with a lower IQ at toddlerhood ${ }^{5 ; 6}$, though this relation was not observed in older children ${ }^{10 ; 14}$. While we observed some effects of prematurity on executive functioning and GM structure in this thesis, prematurity in this population had in general only a limited impact on brain development. As we excluded major obstetrical and neurological complications, often associated with prematurity ${ }^{223}$, the resulting findings might depict a rather optimistic view on the effects of prematurity. On the other hand, while neurocognitive development of very preterm born children is well-documented, literature on late preterm born children, being the majority of preterm cases in this cohort, show more subtle effects ${ }^{70-72}$. In the case of cancer during pregnancy, late preterm labour induction can be required when the fetal or maternal condition is 
deteriorating, or when the required oncological treatment includes risks for the fetus that outbalance the risks of late prematurity ${ }^{223}$.

As chemotherapy and prematurity fail to explain the observed differences in grey and white matter structure (chapter 6 ), clearly other factors do play a role. Moreover, similar to other studies by Menning et al. ${ }^{182}$ and Andreotti et al. ${ }^{181}$, in the work of chapter 4 , where we studied longitudinal brain volume changes in patients with breast cancer, we also found brain volume changes in patients that did not receive chemotherapy. Psychological factors such as stress, anxiety and depression ${ }^{20 ; 180}$ have shown to play an important role in the cognitive decline in cancer survivors. Even more so, the psychosocial impact of a cancer diagnosis during pregnancy, can not be underestimated ${ }^{14 ; 82 ; 224}$. Multiple studies have shown that maternal stress ${ }^{59 ; 63 ; 75 ; 76}$, anxiety $^{59 ; 77}$, depression ${ }^{59 ; 63 ; 75 ; 78 ; 79}$, can alter neurodevelopment of the child. The intense and stressful experience of undergoing cancer therapy during pregnancy might increase maternal cortisol levels during a long period of the pregnancy. This in turn might alter foetal neuronal planning, affecting long-term cognitive and behavioural functioning ${ }^{63}$. Moreover, post-partum maternal psychological distress and associated decreased maternal bonding can affect cognitive and behavioural development of the child ${ }^{82 ; 224-226}$. In particular, decreased maternal bonding and maternal depressive symptoms have been found to affect the child's verbal development, which might explain our findings of altered gyrification of the left superior temporal gyrus (chapter 6) and findings of decreased verbal IQ in children born after cancer-complicated pregnancies ${ }^{14}$. As such, psychological and social support of families confronted with cancer during pregnancy might benefit both mother and child ${ }^{59 ; 60}$.

However, specific chemotherapeutic agents and therapeutic decisions might affect neurodevelopment of the child after cancer-complicated pregnancies. The findings of chapter 6 described the potential risks of platinum derivatives during pregnancy on gyrification of the left superior temporal gyrus, whereas anthracycline-based combination treatments indicated a more favorable outcome on white matter microstructural development compared to other chemotherapy regimens. While this shows that different chemotherapeutics during pregnancy can affect the developing brain differently, heterogeneity in treatment during pregnancy and the limited sample size of children with prenatal chemotherapy exposure in the studies in chapters 5 and 6 (resp. $\mathrm{n}=20$ and $\mathrm{n}=30$ ) makes the current studies underpowered for distinguishing these effects. However, it has been shown that some chemotherapeutic agents have higher transplacental transfer than others ${ }^{8 ; 9 ; 11}$. Moreover, some chemotherapeutic agents, e.g. 5-Fluoruracil, cisplatin and methothrexate, might cross the blood-brain barrier more easily, causing direct neurotoxicity, whereas other chemotherapeutics do not cross as easily and affect the fetal brain through indirect mechanisms such as neuro-inflammation and oxidative stress $21 ; 37 ; 42 ; 44$. Next to this, the immature fetal blood-brain barrier and metabolism, along with fetal reabsorption of excreted 
urine, might alter neurotoxic effects of different agents compared to those observed in cancer survivors. Finally, radiotherapy during pregnancy was not indicated as a risk factor, but was only administered during pregnancy in 5 out of 42 cases in chapter 6 .

To conclude, structural and functional evaluation of brain development in 9 year old children that had been in utero exposed to cancer treatment is overall reassuring. Subtle differences are noted in development of executive function, as well as in grey and white matter structure. However, the differences observed in this project are often not explained by the use of chemotherapy during pregnancy. As such we conclude that, based on our findings, there is no contraindication for the use of chemotherapy in the $2^{\text {nd }}$ and $3^{\text {rd }}$ trimester of pregnancy. However, we do emphasize the need for more long-term follow up data. Clinical and preclinical research should focus on both treatment-related and secondary mechanisms (inflammation, anemia, stress, anxiety, maternal death, ...) affecting neurodevelopment when cancer is diagnosed, and treated, during pregnancy.

\subsection{Limitations}

The research presented throughout this dissertation is bound to certain limitations, both inherent to the studied population as well as the used methodology. The following paragraphs detail some of these limitations.

Cancer in pregnancy is a rare and heterogeneous co-morbidity. This leads to several inherent limitations. First, the study acquired data over a long period of time, which increased the risk of signal drift in the MRI or ERP set-up. In order to limit this effect, the test setup remained constant over the whole duration of this research and controls were recruited as much as possible concurrently with test subjects. However, as a result we could not implement any recent improvements, such as multiband acceleration for MRI acquisition, during the progress of this study. Secondly, we could only acquire data of a limited group of children, decreasing the statistical power. This way, certain effects might be missed in current analyses. Third, mothers were diagnosed with a variety of tumours during pregnancy and received different therapies. While this improves the generalizability of the observed effects, it might hamper their interpretability. Moreover, as a variety of secondary effects might play a role in the observed neural impact, it becomes very challenging to disentangle all these potential sources.

In chapters 5 and 6 , we used a matched control group to disentangle effects of prematurity and cancer in pregnancy. However, we excluded many neurological co-morbidities, which are common in premature children. Although not all of these co-morbidities might be as common in children born out of cancer-complicated pregnancies this might have resulted in a positive bias in the 
premature groups. Moreover, while we controlled for prematurity, it is impossible to control for all social and environmental factors. Furthermore, this discrepancy between groups might be amplified by a participation bias, as motivation to participate in this study might differ between study and control group children.

A clear strength of this study is that it is embedded within the INCIP registration and follow-up studies. This enabled us to relate neuroimaging findings to the psychological outcome of the children and clinical history of the mothers. However, while these datasets provide extensive information on both mother and child, they also have certain limitations. For instance, data on the neuropsychological impact of the cancer during pregnancy ${ }^{82 ; 224}$ on the mother, e.g. concerning anxiety, stress and depression, are currently not acquired for all patients.

Only a handful of studies have investigated neurocognitive outcome after maternal cancer during pregnancy ${ }^{5-7 ; 10 ; 14 ; 17}$. Moreover, most of these studies are part of the same international INCIP follow-up and often contain partially overlapping populations, albeit often observed at different ages. While such international approach increases generalizability and improves statistical power, systematic biases in design and methodology of this study might affect the observed effects in these studies. Furthermore, only one imaging study has previously looked into neurodevelopment after prenatal exposure to chemotherapy ${ }^{17}$. This makes the current results highly exploratory. Future replication studies in other, larger cohorts should confirm the findings in this dissertation.

As mentioned in chapter 3, performing MRI and ERP assessments in children is especially challenging. This study has minimized potential motion-induced noise and bias, by ensuring children enter these test environments in a comfortable manner, as well as through state-of-the art data processing and data quality assurance procedures. However, remaining effects of motion can still have affected outcomes in this study.

Multimodal neuroimaging offer a great opportunity in investigating brain development. However, these measurement only observe these functions through indirect measures. For instance, ERP only measure a mixture of neural electric activity at the scalp, rs-fMRI measure brain activity through the effect the BOLD-signal has on the T2*-weighted contrast and HARDI methods probe white matter microstructure through signal loss in the gradient-weighted images by anisotropic Brownian motion of the hydrogen protons. Moreover, all these techniques are impacted by motion, background noise and bias, imperfections in the equipment and physiological activity. For this reason, findings of multimodal neuroimaging should be interpreted together with the neuropsychological, preclinical and clinical findings in this population.

In order to gain quantitative information from the acquired MRI and ERP data, the data undergoes multiple steps of processing. While preprocessing steps are intended to remove sources 
of bias and noise, imperfections in these steps might introduce artificial bias as well. Throughout this thesis we implemented as much as possible state-of-the-art published pipelines ${ }^{108 ; 125 ; 154}$, only deviating from standard settings if we had clear indications this would better suit our data. Next, Most MR analyses need the registration of images to a common space and segmentation into CSF, WM and GM. As both steps suffer from imperfections, and can be affected by noise, bias or artefacts in the data ${ }^{148}$, these steps can also affect results. Throughout this thesis, we have avoided going to a common space whenever possible or used a population-derived atlas. In a last image processing step, often a modelling of the data is performed to extract quantitative measures. However, these models have to make certain assumptions on the data and by consequence can only estimate the actual brain parameters of interest. Moreover, most often multiple models and implementations of these models exist, which might result in different estimations. In this work we aimed to use models that were openly available, state-of-the-art, commonly used and provide stable measures and used few assumptions. By using models that fitted these requirements as much as possible, we tried to maximize the generalizability and interpretability of our findings. Finally, to ensure proper execution of all image processing steps, we implemented multiple visual and quantitative data quality checks between different crucial steps.

\subsection{Future perspectives}

While the work in this dissertation establishes the relation between prenatal exposure to maternal cancer treatment and neurodevelopment, still many questions remain largely unanswered. The following paragraphs contain suggestions for how future research might explore these questions

First of all, due to the heterogeneity in cancer-complicated pregnancies, the study could not disentangle the neural effects of specific clinical decisions and secondary effects. This calls for larger datasets. While acquiring data for a longer period of time might partially solve this issue it still might fall short in achieving a large enough dataset. Moreover, changes in clinical practice throughout this period, inevitable changes in scanner set-up and generational differences might also partially hamper interpretation. International multicenter data acquisition, which is already in place in the INCIP network for the psychologic and pediatric follow-up of these children ${ }^{6 ; 14}$, might provide another solution. Large-scale imaging initiatives such as the Alzheimer's Disease Neuroimaging Initiative ${ }^{127}$, the human connectome project ${ }^{227}$ and The Adolescent Brain Cognitive Development study ${ }^{228}$ have proven the feasibility of large-scale multicenter imaging acquisition. However, such studies demand a high dedication of all participating centers, including harmonization of scanner protocols and use of costly infrastructure and trained personnel, and often employ methods such as travelling human participants and phantoms to account for 
differences between scanners over time. Even when such approaches are used, combining data from multiple scanners remains challenging today $51 ; 105$.

Pre-clinical research could provide another solution for disentangling the neural effects of different aspects of prenatal exposure to cancer treatment. In the past, one study has already investigated the effect of prenatal exposure to vinblastine and doxorubicin on neurodevelopment in mice, observing effects of these agents on foetal brain development and offspring behaviour ${ }^{15}$. While translation of animal brain and pregnancy models might not always be straightforward, it can provide a platform for testing hypotheses from clinical studies, such as the work in this dissertation, which are often underpowered due to heterogeneity in the population and multiple confounding factors. For instance, Winocur and colleagues ${ }^{166}$ disentangled effects of chemotherapy (Methothrexate and 5FU) and tumor (breast) on brain volume in a mouse model, providing valuable, independent, complementary information to the observed effects in chapter 4 .

This dissertation focussed on neurodevelopment after prenatal exposure to maternal cancer. Earlier research also observed, although often subclinical, relations between chemotherapy during pregnancy and birth weight, as well as heart functioning ${ }^{6}$. Longer term follow-up will be necessary to investigate potential effects of prenatal exposure to cancer therapy on e.g. the risk of developing cancer in later life, gonadal development and fertility. Such long-term multidisciplinary collaboration will be necessary for obtaining a general understanding on the consequences of cancer treatment during pregnancy. These insights will be essential to allow patients and clinicians to make well-informed decisions.

While long-term follow-up studies provide critical information on the long-term safety of cancer treatment during pregnancy, they cannot capture the effects of advances in clinical practice. For instance, proton therapy, as compared to conventional photon therapy, has been indicated as potentially safer radiotherapeutic intervention during pregnancy ${ }^{229 ; 230}$. Preclinical and computational models are one way of predicting the impact such changes might have on the foetus. However, these models can only approximate the actual effects in patients. For this reason, these methods always need clinical validation. Registration of clinical outcomes and short-term follow-up could indeed capture short-term effects, but might only have limited predictive value for later life neurocognitive outcome ${ }^{231}$.

Foetal and neonatal multimodal MRI might provide additional information on the neurocognitive impact of prenatal exposure to cancer treatment, potentially increasing the predictive value of short-term assessments on later-life outcome. Moreover, this way it is even possible to observe brain development closer to the impact of cancer therapy, avoiding many potential secondary effects. So far, only one study examined the effects of chemotherapy during pregnancy on 
neonatal brain volume ${ }^{17}$. However, recent advances in foetal and neonatal neuroimaging have made it possible to study the developing brain with more detail ${ }^{46 ; 232}$. Such techniques have allowed to study WM microstructural ${ }^{233}$, functional ${ }^{234}$ and cortical neurodevelopment ${ }^{235}$ in both foetuses and neonates.

In chapter 6, we used multiple MR modalities to investigate brain development. However, in order to avoid extensive motion artefacts and ensure a comfortable experience for the participants, scan time per patient was limited to about 30 minutes, leading to a subset of potential MR modalities being acquired. Other MR modalities could potentially provide additional insights. For instance myelin water imaging could refine findings from diffusion-weighted imaging results, by estimating the differences in myelination ${ }^{236}$. Another example is MR spectroscopy. This family of MR techniques allows the estimation of different metabolic compounds in the brain ${ }^{237}$. One possible application of these methods for this population could be the study of the neurotransmitter gamma-aminobutyric acid (GABA) ${ }^{238}$. Due to their protracted development, GABA-ergic interneurons might be particularly vulnerable to a perinatal impact ${ }^{239}$.

Finally, most important to the work in this thesis is the translation of its findings on neurodevelopment after maternal cancer during pregnancy into daily clinical practice. While the findings in this dissertation do not contraindicate the use of chemotherapy, surgery or radiotherapy during pregnancy, they did observe an effect of prenatal exposure to maternal cancer (therapy) on the later life neurodevelopment. Multiple studies have discussed the effect psycho-social impact a diagnosis of cancer during pregnancy has on young families ${ }^{82 ; 224}$, which might contribute to the observed differences in neurodevelopment here. Through perinatal support and early interventions, such as environmental enrichment within the neonatal intensive care unit, both psychological well-being of the parents and neurodevelopment of the child could be promoted ${ }^{59 ; 60}$.

\subsection{General conclusion}

The work in this dissertation observed a subtle impact of prenatal exposure to maternal cancer and its therapy on neurodevelopment at the age of nine, not limited to the effect of prematurity. These findings resonate with earlier subtle findings on neuropsychological development in this population ${ }^{5 ; 14}$. With exception of platinum derivatives, chemotherapy during pregnancy could not be directly identified as a risk factor for altered neurodevelopment. Rather, the psychological impact of a cancer diagnosis during pregnancy might partially explain these findings, emphasizing the need for a good psychosocial support system for mothers with cancer during pregnancy and their families. Balancing the benefits of treating cancer during pregnancy against the subtle 
neurocognitive impact observed in this thesis, the current data favour cancer treatment during pregnancy, including chemotherapy, when clinically indicated. 
CHAPTER

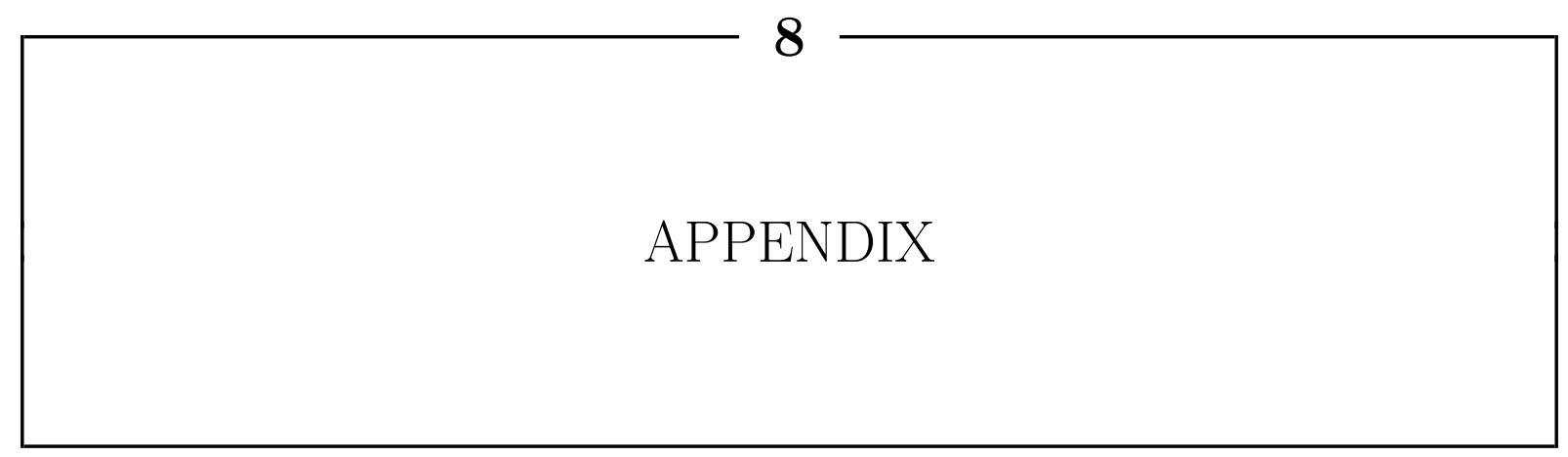


8.1 Supplementary tables to chapter 4 


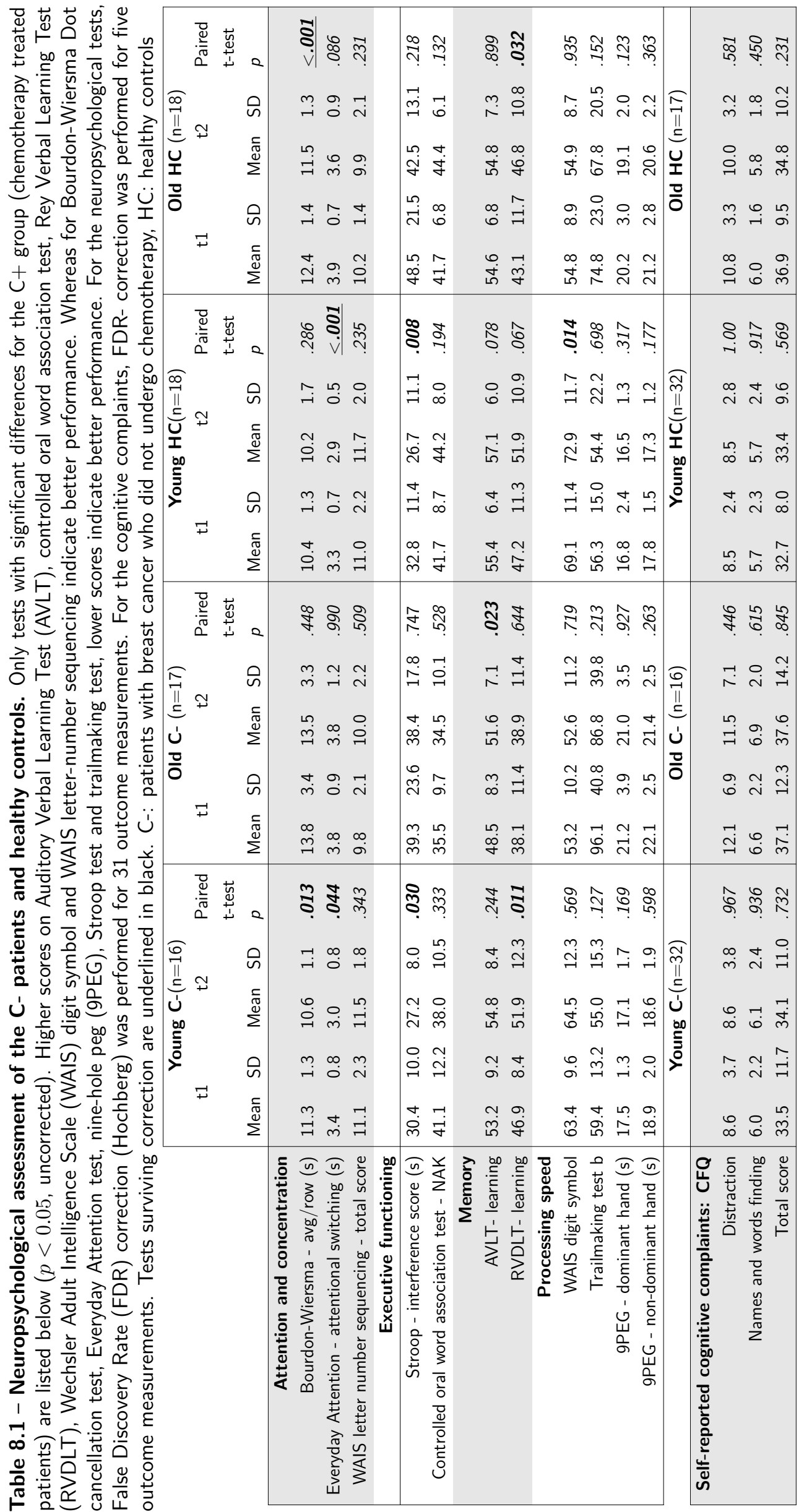


Table 8.2 - MNI coordinates, cluster extent and statistical values of significant clusters from the DBM analysis described in Figure 4.1. Statistics are performed at three levels: set level (indicating chance of finding this number of clusters), cluster level and peak level. Significance in this study was assessed on cluster level for a Bonferroni-correct $p<.05 . p_{F W E}$ : Bonferroni corrected $p ; q_{F D R}$ : false discovery rate corrected $q$; $K_{E}$ : Cluster extent (in voxels); $c$ : number of clusters found at $p<.001$; t1: timepoint 1 ; t2 timepoint 2; C+: patients breast cancer who underwent chemotherapy; C-: patients with breast cancer who did not undergo chemotherapy; HC: healthy controls

\begin{tabular}{|c|c|c|c|c|c|c|c|c|c|c|c|c|c|}
\hline \multirow[t]{2}{*}{ Contrast } & \multicolumn{2}{|c|}{ Set level } & \multicolumn{4}{|c|}{ Cluster level } & \multicolumn{4}{|c|}{ Peak level } & \multicolumn{3}{|c|}{ MNI Coordinates (mm) } \\
\hline & $p$ & $c$ & $p_{F W E}$ & $q_{F D R}$ & $K_{E}$ & $P_{\text {uncorr }}$ & $p_{F W E}$ & $q_{F D R}$ & $T$ & $P_{\text {uncorr }}$ & $x$ & $\mathrm{y}$ & z \\
\hline \multirow[t]{8}{*}{$C+: t 1-t 2$} & .000 & 3 & .000 & .000 & 6554 & .000 & .000 & .001 & 6.23 & .000 & 4.5 & 54 & 18 \\
\hline & & & & & & & .002 & .011 & 5.58 & .000 & -3 & 60 & -6 \\
\hline & & & & & & & .003 & .013 & 5.44 & .000 & -3 & 51 & 19.5 \\
\hline & & & .000 & .000 & 5380 & .000 & .013 & .023 & 5.12 & .000 & 1.5 & 16.5 & 13.5 \\
\hline & & & & & & & .021 & .024 & 4.99 & .000 & 7.5 & 4.5 & 4.5 \\
\hline & & & & & & & .079 & .063 & 4.64 & .000 & -9 & 21 & 9 \\
\hline & & & .013 & .025 & 1102 & .002 & .068 & .058 & 4.68 & .000 & 3 & -63 & 48 \\
\hline & & & & & & & .590 & .329 & 3.92 & .000 & 7.5 & -72 & 43.5 \\
\hline \multirow[t]{11}{*}{$\mathrm{C}+: \mathrm{t} 2-\mathrm{t} 1$} & .000 & 4 & .023 & .019 & 956 & .003 & .007 & .041 & 5.26 & .000 & -13.5 & -49.5 & -34.5 \\
\hline & & & & & & & .532 & .282 & 3.97 & .000 & -16.5 & -34.5 & -37.5 \\
\hline & & & .000 & .000 & 5928 & .000 & .110 & .142 & 4.54 & .000 & 27 & -66 & 19.5 \\
\hline & & & & & & & .185 & .142 & 4.38 & .000 & 28.5 & -58.5 & -3 \\
\hline & & & & & & & .225 & .142 & 4.31 & .000 & 13.5 & -78 & -7.5 \\
\hline & & & .000 & .000 & 5267 & .000 & .133 & .142 & 4.48 & .000 & -22.5 & 9 & 37.5 \\
\hline & & & & & & & .200 & .142 & 4.35 & .000 & -27 & 1.5 & 15 \\
\hline & & & & & & & .259 & .144 & 4.26 & .000 & -22.5 & 9 & -7.5 \\
\hline & & & .007 & .008 & 1269 & .001 & .234 & .142 & 4.30 & .000 & 25.5 & 10.5 & 15 \\
\hline & & & & & & & .267 & .144 & 4.25 & .000 & 22.5 & 4.5 & -6 \\
\hline & & & & & & & .769 & .436 & 3.76 & .000 & 22.5 & 19.5 & 1.5 \\
\hline \multirow[t]{15}{*}{$C-: t 1-t 2$} & .000 & 5 & .000 & .000 & 27444 & .000 & .001 & .021 & 5.76 & .000 & -34.5 & -48 & -36 \\
\hline & & & & & & & .003 & .022 & 5.46 & .000 & -16.5 & 19.5 & 18 \\
\hline & & & & & & & .010 & .048 & 5.18 & .000 & -24 & -70.5 & -36 \\
\hline & & & .000 & .000 & 2972 & .000 & .004 & .022 & 5.43 & .000 & 9 & 73.5 & 4.5 \\
\hline & & & & & & & .027 & .075 & 4.93 & .000 & 6 & 51 & 3 \\
\hline & & & & & & & .063 & .116 & 4.70 & .000 & -4.5 & 55.5 & 1.5 \\
\hline & & & .000 & .001 & 2125 & .000 & .023 & .075 & 4.97 & .000 & 48 & 24 & -1.5 \\
\hline & & & & & & & .092 & .130 & 4.59 & .000 & 34.5 & 22.5 & 0 \\
\hline & & & & & & & .198 & .157 & 4.35 & .000 & 51 & -37.5 & 9 \\
\hline & & & .017 & .025 & 1033 & .002 & .058 & .114 & 4.72 & .000 & 7.5 & -72 & 13.5 \\
\hline & & & & & & & .382 & .205 & 4.12 & .000 & 9 & 57 & 18 \\
\hline & & & & & & & .536 & .272 & 3.97 & .000 & 13.5 & -91.5 & 3 \\
\hline & & & .010 & .019 & 1171 & .001 & .568 & .280 & 3.94 & .000 & 22.5 & -10.5 & 21 \\
\hline & & & & & & & .626 & .294 & 3.89 & .000 & 18 & 3 & 18 \\
\hline & & & & & & & .967 & .663 & 3.45 & .000 & 22.5 & -4.5 & 28.5 \\
\hline C-: t2-t1 & .067 & 1 & .001 & .001 & 1920 & .000 & .003 & .004 & 5.46 & .000 & -3 & -33 & 52.5 \\
\hline
\end{tabular}


Table 8.3 - MNI coordinates, cluster extent and statistical values of significant clusters from the DBM analysis described in Figure 4.2. Statistics are performed at three levels: set level (indicating chance of finding this number of clusters), cluster level and peak level. Significance in this study was assessed on cluster level for a Bonferroni-correct $p<.05 . p_{F W E}$ : Bonferroni corrected $p ; q_{F D R}$ : false discovery rate corrected $q$; $K_{E}$ : Cluster extent (in voxels); $c$ : number of clusters found at $p<.001$; t1: timepoint 1 ; t2 timepoint 2 ; $\mathrm{C}+$ : patients breast cancer who underwent chemotherapy; C-: patients with breast cancer who did not undergo chemotherapy; HC: healthy controls; y: young, pre-menopausal subgroup; o: old, post-menopausal subgroup

\begin{tabular}{|c|c|c|c|c|c|c|c|c|c|c|c|c|c|}
\hline \multirow[t]{2}{*}{ Contrast } & \multicolumn{3}{|c|}{ Set level } & \multicolumn{3}{|c|}{ Cluster level } & \multicolumn{4}{|c|}{ Peak level } & \multicolumn{3}{|c|}{ MNI Coordinates $(\mathrm{mm})$} \\
\hline & $p$ & $c$ & $p_{F W E}$ & $q_{F D R}$ & $K_{E}$ & $P_{\text {uncorr }}$ & $p_{F W E}$ & $q_{F D R}$ & $T$ & $P_{\text {uncorr }}$ & $x$ & $y$ & z \\
\hline \multirow[t]{13}{*}{$C+{ }_{y}: t 1-t 2$} & \multirow[t]{13}{*}{.000} & \multirow[t]{13}{*}{6} & \multirow[t]{3}{*}{.000} & \multirow[t]{3}{*}{.000} & 10044 & .000 & .000 & .000 & 6.91 & .000 & 4.5 & 52.5 & 16.5 \\
\hline & & & & & & & .000 & .000 & 6.27 & .000 & -3 & 60 & -7.5 \\
\hline & & & & & & & .000 & .000 & 6.15 & .000 & -3 & 51 & 19.5 \\
\hline & & & .000 & .000 & 10557 & .000 & .000 & .000 & 6.61 & .000 & 0 & 7.5 & 18 \\
\hline & & & & & & & .000 & .000 & 6.25 & .000 & 1.5 & 4.5 & 7.5 \\
\hline & & & & & & & .000 & .001 & 5.91 & .000 & -9 & 19.5 & 10.5 \\
\hline & & & .049 & .041 & 774 & .007 & .000 & .001 & 5.96 & .000 & -30 & -79.5 & 30 \\
\hline & & & .029 & .036 & 905 & .004 & .003 & .003 & 5.49 & .000 & 34.5 & -49.5 & 51 \\
\hline & & & & & & & .664 & .244 & 3.86 & .000 & 43.5 & -51 & 63 \\
\hline & & & .000 & .001 & 2204 & .000 & .004 & .004 & 5.41 & .000 & 3 & -67.5 & 46.5 \\
\hline & & & & & & & .022 & .014 & 4.98 & .000 & 9 & -72 & 40.5 \\
\hline & & & & & & & .462 & .184 & 4.04 & .000 & -1.5 & -70.5 & 31.5 \\
\hline & & & .049 & .041 & 775 & .007 & .005 & .005 & 5.33 & .000 & 52.5 & 10.5 & 30 \\
\hline $\mathrm{C}+{ }_{\mathrm{y}}: \mathrm{t} 2-\mathrm{t} 1$ & .001 & 2 & .000 & .000 & 11516 & .000 & .002 & .021 & 5.54 & .000 & -21 & 9 & 37.5 \\
\hline & & & & & & & .024 & .030 & 4.96 & .000 & -25.5 & 1.5 & 15 \\
\hline & & & & & & & .029 & .030 & 4.91 & .000 & -31.5 & 1.5 & 21 \\
\hline & & & .000 & .000 & 15781 & .000 & .025 & .030 & 4.95 & .000 & 27 & -64.5 & 18 \\
\hline & & & & & & & .027 & .030 & 4.93 & .000 & 15 & -76.5 & -9 \\
\hline & & & & & & & .075 & .057 & 4.65 & .000 & 22.5 & -54 & 30 \\
\hline $\mathrm{C}+:(\mathrm{t} 2-\mathrm{t} 1)_{\mathrm{o}}-(\mathrm{t} 2-\mathrm{t} 1)_{\mathrm{y}}$ & .006 & 2 & .000 & .000 & 10612 & .000 & .001 & .005 & 5.84 & .000 & 28.5 & -51 & 10.5 \\
\hline & & & & & & & .004 & .010 & 5.42 & .000 & -1.5 & 1.5 & 18 \\
\hline & & & & & & & .004 & .010 & 5.41 & .000 & 0 & 3 & 9 \\
\hline & & & .000 & .001 & 2137 & .000 & .218 & .136 & 4.32 & .000 & -3 & 43.5 & 28.5 \\
\hline & & & & & & & .418 & .230 & 4.08 & .000 & -3 & 30 & 39 \\
\hline & & & & & & & .542 & .274 & 3.97 & .000 & -4.5 & 67.5 & 18 \\
\hline $\mathrm{C}+:(\mathrm{t} 1-\mathrm{t} 2)_{\mathrm{o}}-(\mathrm{t} 1-\mathrm{t} 2)_{\mathrm{y}}$ & .005 & 2 & .032 & .024 & 879 & .004 & .315 & .434 & 4.19 & .000 & 43.5 & -16.5 & -18 \\
\hline & & & & & & & .773 & .434 & 3.75 & .000 & 43.5 & -19.5 & -6 \\
\hline & & & .005 & .008 & 1353 & .001 & .539 & .434 & 3.97 & .000 & 19.5 & 19.5 & 27 \\
\hline & & & & & & & .899 & .519 & 3.60 & .000 & 30 & 36 & 4.5 \\
\hline$C_{-y}: t 1-t 2$ & .001 & 2 & .000 & .000 & 6886 & .000 & .000 & .001 & 6.33 & .000 & -33 & -46.5 & -37.5 \\
\hline & & & & & & & .007 & .020 & 5.27 & .000 & -21 & -69 & -31.5 \\
\hline & & & & & & & .011 & .023 & 5.15 & .000 & -27 & -48 & -45 \\
\hline & & & .000 & .000 & 3083 & .000 & .004 & .015 & 5.44 & .000 & -4.5 & 49.5 & 12 \\
\hline & & & & & & & .016 & .023 & 5.06 & .000 & 9 & 73.5 & 4.5 \\
\hline & & & & & & & .016 & .023 & 5.06 & .000 & 4.5 & 52.5 & 3 \\
\hline$C_{-y}: t 2-t 1$ & .345 & 1 & .025 & .025 & 936 & .004 & .292 & .447 & 4.22 & .000 & 3 & -24 & 57 \\
\hline & & & & & & & .554 & .447 & 3.95 & .000 & -1.5 & -30 & 54 \\
\hline & & & & & & & .571 & .447 & 3.94 & .000 & -3 & -36 & 60 \\
\hline $\mathrm{C}_{-\mathrm{o}}: \mathrm{t} 1-\mathrm{t} 2$ & .000 & 8 & .005 & .008 & 1354 & .001 & .013 & .155 & 5.11 & .000 & -57 & -21 & 39 \\
\hline & & & & & & & .322 & .368 & 4.19 & .000 & -40.5 & -28.5 & 48 \\
\hline & & & .008 & .010 & 1223 & .001 & .054 & .204 & 4.74 & .000 & 4.5 & -78 & 12 \\
\hline & & & & & & & .625 & .388 & 3.89 & .000 & 9 & -79.5 & 33 \\
\hline & & & & & & & .746 & .435 & 3.78 & .000 & 10.5 & -67.5 & 10.5 \\
\hline & & & .040 & .033 & 824 & .006 & .056 & .204 & 4.73 & .000 & 3 & -24 & 31.5 \\
\hline & & & & & & & .485 & .388 & 4.02 & .000 & -4.5 & -34.5 & 31.5 \\
\hline & & & .000 & .000 & 3751 & .000 & .088 & .210 & 4.60 & .000 & -28.5 & -45 & 28.5 \\
\hline & & & & & & & .114 & .226 & 4.53 & .000 & -33 & -52.5 & 15 \\
\hline & & & & & & & .231 & .300 & 4.30 & .000 & -46.5 & -70.5 & 28.5 \\
\hline & & & .000 & .001 & 2205 & .000 & .146 & .226 & 4.45 & .000 & -63 & -42 & 24 \\
\hline & & & & & & & .500 & .388 & 4.00 & .000 & -55.5 & -30 & 19.5 \\
\hline & & & & & & & .615 & .388 & 3.90 & .000 & -39 & -13.5 & 18 \\
\hline & & & .001 & .002 & 1834 & .000 & .223 & .300 & 4.31 & .000 & -46.5 & 18 & 51 \\
\hline & & & & & & & .567 & .388 & 3.94 & .000 & -39 & 4.5 & 40.5 \\
\hline & & & & & & & .776 & .451 & 3.75 & .000 & -16.5 & 9 & 49.5 \\
\hline & & & .018 & .018 & 1019 & .003 & .396 & .376 & 4.10 & .000 & -18 & 0 & 28.5 \\
\hline & & & & & & & .626 & .388 & 3.89 & .000 & -18 & 21 & 18 \\
\hline & & & & & & & .686 & .390 & 3.84 & .000 & -6 & 4.5 & 28.5 \\
\hline & & & .050 & .037 & 769 & .007 & .396 & .376 & 4.10 & .000 & -22.5 & 10.5 & -4.5 \\
\hline & & & & & & & .512 & .388 & 3.99 & .000 & -12 & 15 & 1.5 \\
\hline$C-:(t 1-t 2)_{0}-(t 1-t 2)_{y}$ & .067 & 1 & .012 & .032 & 1137 & .002 & .889 & .870 & 3.61 & .000 & -30 & 28.5 & 1.5 \\
\hline & & & & & & & .933 & .870 & 3.54 & .000 & -25.5 & 33 & 25.5 \\
\hline & & & & & & & .999 & .952 & 3.16 & .001 & -16.5 & 31.5 & 13.5 \\
\hline
\end{tabular}




\subsection{Supplementary materials to chapter 6}

Table 8.4 - Image quality metrics. All metrics are calculated on the groups after exclusion criteria were applied: $\mathrm{n}=42$ per group for $\mathrm{rs}-\mathrm{fMRI}$ and diffusion images and $\mathrm{n}=38$ per group for T1 images. rs-fMRI: resting-state functional MRI. Q1: first quartile. Q3: third quartile.

\begin{tabular}{|rcccccc|}
\cline { 2 - 7 } \multicolumn{1}{c|}{} & \multicolumn{4}{c|}{ Study group } & \multicolumn{3}{c|}{ Control group } \\
& Median & Q1 & Q3 & Median & Q1 & Q3 \\
\hline T1-weighted & & & & & & \\
Contrast-to-noise ratio & 5.25 & 5.07 & 5.42 & 5.18 & 5.04 & 5.44 \\
Entropy focus criterion & 0.604 & 0.586 & 0.671 & 0.622 & 0.562 & 0.739 \\
rs-fMRI & & & & & & \\
Mean framewise displacement (mm) & 0.215 & 0.164 & 0.275 & 0.197 & 0.164 & 0.264 \\
Temporal signal-to-noise ratio & 61.6 & 51.7 & 74.4 & 60.4 & 49.7 & 76.0 \\
Diffusion & & & & & & \\
Mean translation between slices (mm) & 0.302 & 0.273 & 0.350 & 0.297 & 0.278 & 0.342 \\
Mean rotation between slices (degrees) & 0.243 & 0.221 & 0.294 & 0.248 & 0.220 & 0.283 \\
Outlier ratio & 0.079 & 0.058 & 0.100 & 0.076 & 0.060 & 0.092 \\
\hline
\end{tabular}

Table 8.5 - Global brain volumes. Brain volumes are expressed in ml. Significance is assessed at $p<.05$.

\begin{tabular}{|cc|cccccccc|}
\hline Study group & Parameter & \multicolumn{3}{|c}{ Study group } & \multicolumn{2}{c}{ Control group } & \multicolumn{3}{c|}{ P FDR } \\
& & & Mean & SD & Mean & SD & Group & GA & Group \\
& & & & & & & by GA \\
\hline All cancers $(n=38)$ & Total brain volume & 1205 & 113 & 1239 & 85 & .143 & .898 & .666 \\
All cancers $(n=38)$ & Grey matter & 732 & 68 & 755 & 49 & .090 & .650 & .947 \\
All cancers $(n=38)$ & White matter & 473 & 48 & 483 & 41 & .298 & .751 & .374 \\
Chemo $(n=27)$ & Total brain volume & 1208 & 123 & 1238 & 90 & .315 & .467 & .933 \\
Chemo $(n=27)$ & Grey matter & 737 & 73 & 754 & 51 & .310 & .836 & .744 \\
Chemo $(n=27)$ & White matter & 471 & 52 & 483 & 43 & .355 & .167 & .526 \\
\hline
\end{tabular}




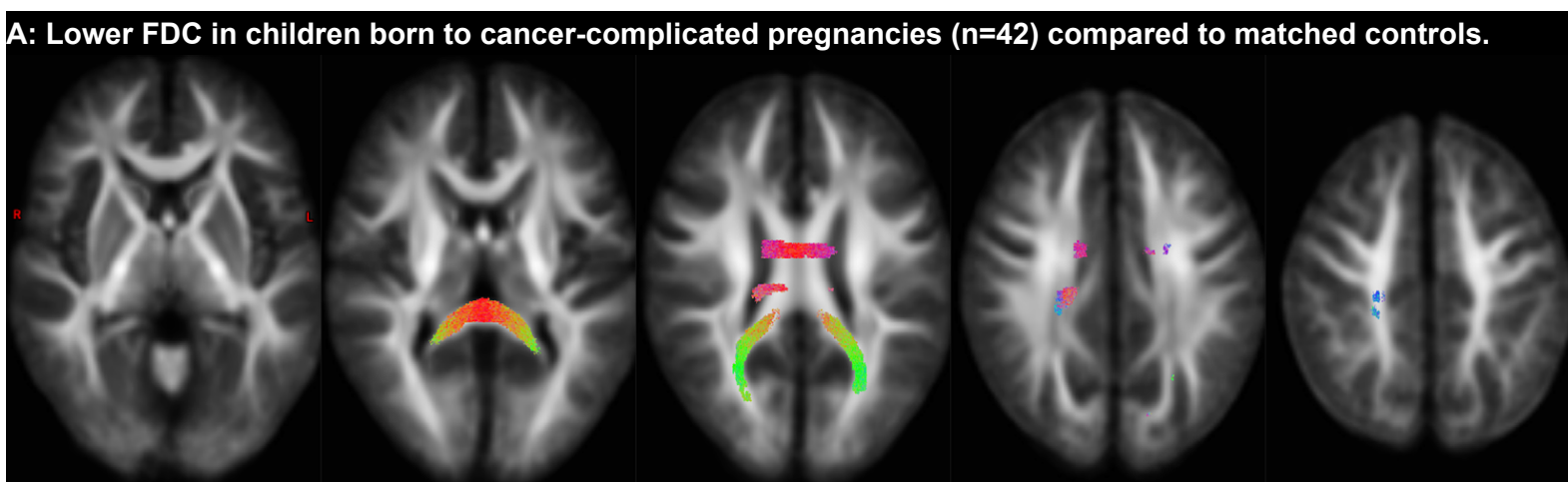

B: Higher FCS in children born to cancer-complicated pregnancies $(n=42)$ compared to matched controls.

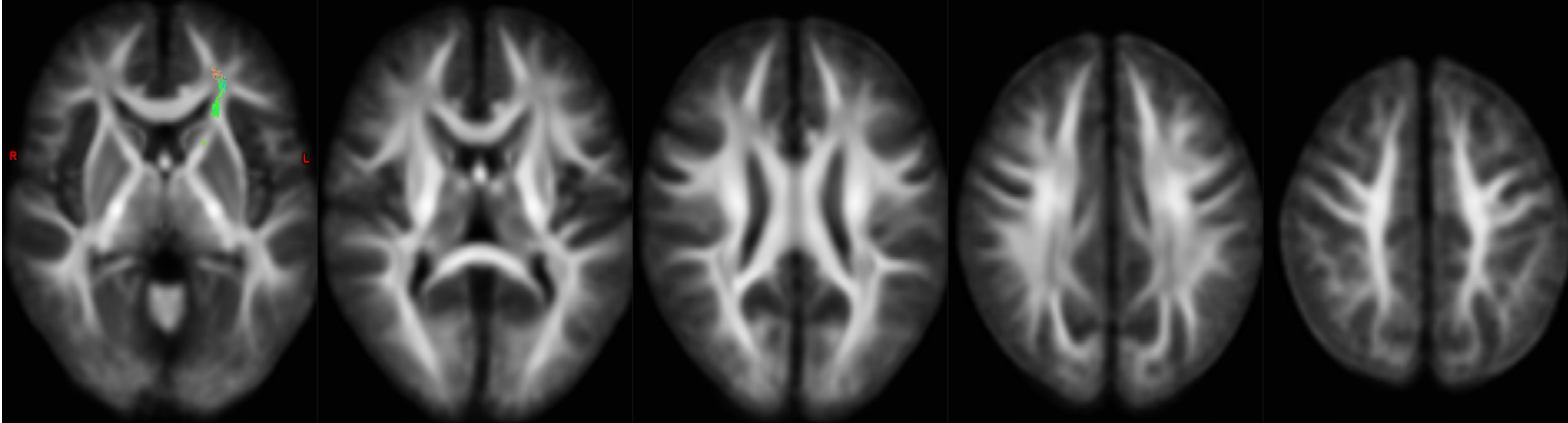

C: Lower FCS in children with prenatal chemotherapy exposure $(n=30)$ compared to matched controls.

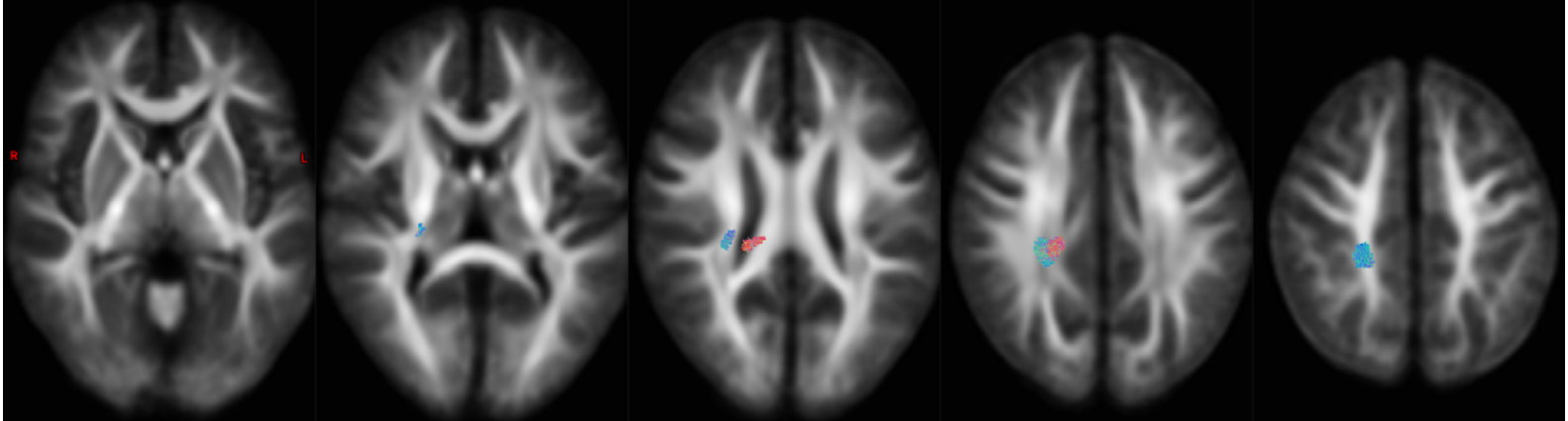

D: Lower FDC in children with prenatal chemotherapy exposure $(n=30)$ compared to matched controls.

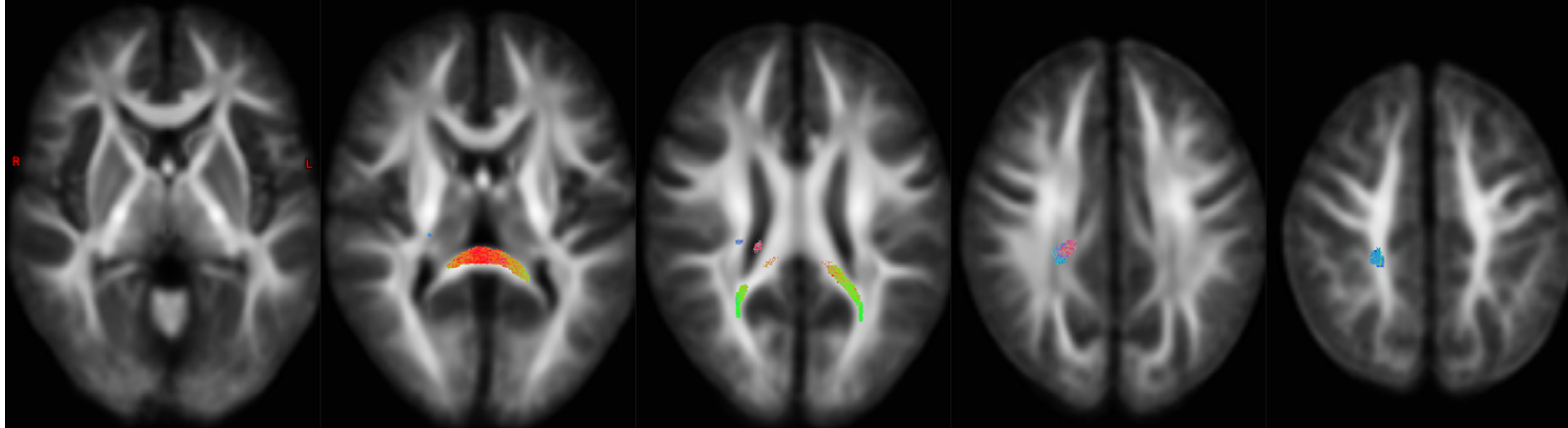

E: Higher FDC in children with prenatal chemotherapy exposure $(n=30)$ compared to matched controls.

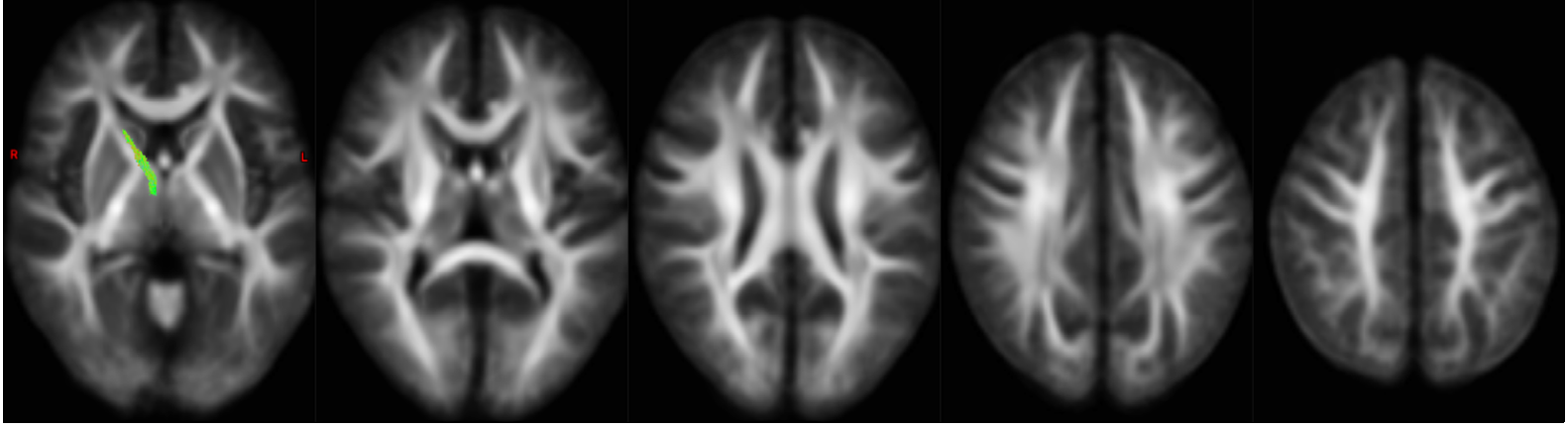

Figure 8.1 - Observed differences in WM microstructure at trend level. Trends for the effects in figure 6.2 are visualized at $p<.1$, using connectivity-based fixel enhancement ${ }^{209}$ with family-wise-error correction. All results are rendered with 200000 streamlines (as described earlier ${ }^{125}$ ), mapped on $10 \mathrm{~mm}$ interval slices and coloured with conventional directional colour encoding. FCS: fibre cross-section. FDC: Fibre density and cross-section. 


\subsubsection{Data analysis}

All images were processed by combining publicly available toolboxes and in-house developed scripts (using bash and Matlab v2018b). Unless otherwise mentioned, standard settings were used. Prior to processing, all images were converted to the Brain Imaging Data Structure (BIDS) 240 and visually inspected for issues. Quantitative quality parameters of the T1-weighted images and rs-fmri were calculated using MRIQC (v0.14.2) $)^{112}$ and using shardrecon ${ }^{109}$ for the diffusion-weighted images. For the T1-weighted images, the following quality parameters are reported: contrast-to-noise ratio $(\mathrm{CNR})^{241}$, coefficient of joint variation (CJV) ${ }^{242}$ and entropy focus criterion (EFC) ${ }^{243}$. For rs-fMRI mean framewise displacement (FD) ${ }^{244}$ and temporal signal-to-noise ratio (tSNR) ${ }^{245}$ are reported. For diffusion images mean Euclidean framewise translation and rotation, as well as the outlier ratio (OR) were used, all using the estimates from the SHARD-recon motion correction ${ }^{109}$. The OR was defined as $1-\frac{\sum \text { slice weights }}{\text { Total number of slices }}$. Image quality metrics are provided in Supplementary table 1 . Further visual checks of data quality were performed at multiple steps of the data processing.

\subsubsection{Cortical and subcortical morphometry}

Cortical and subcortical grey matter features were extracted from the T1-weighted images using Mindboggle (v1.3.8) ${ }^{106}$. This analysis hybridizes parcellations of the Freesurfer (v6.0.0) ${ }^{129}$ and ANTs (v2.3.1) cortical thickness ${ }^{153 ; 246}$ pipelines. While this method corrects for underestimation of GM on the outside of the brain, it might be prone to misclassification of dura matter as GM. For this reason, the standard brain mask in Freesurfer was replaced in this analysis by a mask generated by HD-BET ${ }^{247}$. Similarly, this brain mask was applied to the ANTs segmentation. N4 biasfield correction (ANTs v2.3.1) ${ }^{153}$ was applied on the initial T1-weighted image prior to Freesurfer parcellation.

Images scoring in the worst 20 percentile of CJV,CNR or EFC $(n=30)$ were visually rated by a trained radiologist $(A R)$ into bad $(n=1)$, doubtful $(n=16)$ or good $(n=23)$ quality ${ }^{248}$. Images of initially doubtful quality were in-depth re-assessed after surface reconstruction was performed, those with initial bad quality $(n=1)$, were rejected. For each image with doubtful quality, parcellated images were again rated for errors by the same neuroradiologist (AR), and either accepted or rejected for morphometrical analysis. All other surface reconstructions and parcellations were evaluated for major issues, using $4 \mathrm{~mm}$ interleaved axial slices. Participants who were matched to children excluded from the morphometrical analysis, were re-matched to one another when meeting the matching criteria. All reconstructions were deemed of sufficient quality for use in the connectome analyses (including those excluded from the morphometrical analysis). 
The corrected Freesurfer Desikan-Killiany parcellations ${ }^{249 ; 250}$ were used for region-based statistical analysis. GM (subcortical+cortical), WM and total brain (GM+WM) volumes were compared between groups, as well as the volume of the different subcortical structures ${ }^{250}$. Different mean cortical features ${ }^{106}$ of each region were investigated: mean thickness (Freesurfer), surface area, mean travel depth and mean curvature. Statistical analysis was performed within Matlab, using the 'anovan' function. The Benjamini-Hochberg procedure was used to control for multiple comparisons of cortical features in 62 regions and subcortical volumes in 16 regions. For subcortical volume analyses, total brain volume was included as a covariate.

\subsubsection{White matter microstructure}

White matter microstructure was examined using a fixel-based analysis on the multi-shell diffusion-weighted images, which was performed using MRtrix ${ }^{205}$ (v3.0). Pre-processing consisted of the following steps: denoising ${ }^{202}$, Gibbs ringing removal ${ }^{203}$, distortion correction ${ }^{204}$ (FSL v5.0.11 topup, between b0 and reversed phase image, using prior AP padding with 30 voxels), motion correction ${ }^{109}$ (SHARD-recon, $\mathrm{SH}$ basis per shell $=0,4,6$, reduced basis $=2,0$, $\mathrm{reg}=0.005$, zreg=0.001), biasfield correction (ANTs v2.3.1 N4 correction ${ }^{153}$, shrink factor 1 , mesh resolution $=150 \mathrm{~mm}$, spline order 3 , maximum iterations $=500 \times 500$, convergence threshold $=0.0$ ) and upsampling to an isotropic voxel size of $1.3 \mathrm{~mm}$.

Group-average response functions were calculated for WM, GM and CSF. Next, fibre orientation distributions (FOD) were calculated in each voxel using constrained spherical deconvolution (CSD) and Joint bias field correction and intensity normalization was performed. All FOD images were registered to an unbiased population template, which was calculated using data from 15 randomly selected children from both study and control group. Fixel-based parameters of fibre density (FD), fibre cross-section (FCS) and their combined metric of fibre density and cross-section (FDC) were calculated as described elsewhere ${ }^{125}$.

Whole-brain tractography was performed on the template FOD (iFOD2,20 million fibres, maximum angle $=22.5$, minimum $/$ maximum length $=10 / 250 \mathrm{~mm}$, power $=1.0$, FOD cut-off $=.06$ ) and reduced to 2 million fibres using Spherical-deconvolution informed filtering of tractograms $\left(\right.$ SIFT) ${ }^{251}$. Differences in FD, FCS (logarithmically scaled) and FDC were assessed at each individual fixel using connectivity-based fixel enhancement ${ }^{209}$ (CFE). Only fixels where more than 150 streamlines pass through were included in the final analysis. This resulted in the final analysis mask containing 316424 fixels ( $61 \%$ of the original fixel mask). ICV was calculated by back-transforming the group mask to subject-space, as described earlier by Pannek et al. ${ }^{252}$. normalized ICV was used as a covariate in analyses of FCS and FDC ${ }^{208}$.. Significance was inferred at $p<.05$, corrected for multiple corrections using family-wise error correction within 
the CFE framework ${ }^{209}$.

Structural connectomes were generated for each participant. First, the T1-weighted image and corrected Freesurfer parcellation ${ }^{106 ; 249}$ of each participant were registered to first volume of the normalized WM FOD images (ANTs rigid Inter modality Intra subject registration). Next, Whole-brain tractography was performed on normalized WM FOD images (iFOD2, 10 million fibres, minimum $/$ maximum length $=5 / 300 \mathrm{~mm}$, dynamic seeding), using anatomically constrained tractography (ACT, based on the parcellations, including amygdalae and hippocampi as subcortical GM) and SIFT2 re-weighting of streamlines ${ }^{131}$. Finally, weighted undirected connectomes were generated based on SIFT2 re-weighted streamlines and corrected Freesurfer parcellation, with edge values normalized to the maximum edge weight.

\subsubsection{Functional connectivity}

All rs-fMR images were pre-processed using fmriprep (v20.0.1) ${ }^{112}$, with following nonstandard settings: no Freesurfer surface reconstruction, using a fieldmap-less approach for correcting EPI distortion artefacts ${ }^{253}$, calculating motion confounds using ICA-AROMA ${ }^{107}$ (aggressive setting) and using the anatomical image as output space. Next, nuisance regression and temporal band pass filtering $(.009-1 \mathrm{~Hz})$ was applied using the denoiser toolbox (https://github.com/arielletambini/denoiser). This nuisance regression was applied using the following parameters from the fmriprep output: CSF (1 parameter) and WM (1 parameter) signal, framewise translations (3 parameters) and (3 parameters) rotations, ICA-AROMA motion confounds (variable number of parameters) and signal drift (6 parameters). Further analysis was conducted using the CONN toolbox (v19.b) ${ }^{206}$. Within this toolbox, images were segmented (GM, WM and CSF) and normalized to MNI. Next, a Gaussian smoothing kernel of $6 \mathrm{~mm}$ isotropic was applied to the functional images. Based on the a priori hypothesis of possible impact of chemotherapy on executive functioning, nodes of the default mode (DMN), fronto-parietal (FPN), dorsal attention (DAN) and salience networks (SN) were selected from the CONN (v19.b) ${ }^{206}$ network atlas. This resulted in a total of 17 regions included in this analysis. ROI-to-ROI functional coherence between these regions was estimated using bivariate correlations. Significance was assessed at $p<.05$, false discovery rate corrected for comparing 171 connections.

Undirected weighted functional connectomes were generated using absolute bivariate correlations between regions of the corrected Freesurfer parcellation ${ }^{106 ; 249}$ in the mean timeseries of the pre-processed functional images (upsampled to match the anatomical image). 


\subsubsection{4 whole-brain connectomics}

Graph theory analysis was performed using in-house developed MATLAB scripts and the Brain Connectivity toolbox (v2019-03-03). Self-connections were removed from all connectomes. Whole-brain graph theory measures of characteristic path length, global and local efficiency and clustering coefficient ${ }^{207}$ were calculated. Characteristic path length and global efficiency were calculated using Dijkstra's algorithm, with the connection-length matrix defined by the inverse edge weights (except for self-connections which have a zero length). Clustering coefficient and local efficiency measures were calculated as recommended by Wang et al. ${ }^{207}$ For each connectome, 100 random graphs were calculated by randomly permuting the edges, while keeping the connectome symmetry, the zero-weight of the self-connections and excluding graphs with disconnected nodes. Normalized graph measures were calculated by dividing the original graph measures of the connectome by the mean of the graph measures of equivalent random graphs. Statistical comparison of graph measures was performed using standard parametric testing, using SPSS (v19.0) univariate GLM. A Bonferroni correction was introduced for comparing 2 types of graphs. No correction of multiple comparisons was introduced for the number of global graph measures as these are highly interdependent.

\subsubsection{Neurological abnormalities}

Four children presented neurological abnormalities on the MRI, as rated by a clinical neuroradiologist, and had to be excluded from this study. Two children were born to a mother with cancer during pregnancy, with both mothers receiving chemotherapy during pregnancy. The first child, a girl born at 31 weeks of gestation, presented a white matter lesion at the head of right caudate, which could be traced back to a perinatal subependymal bleeding. However, as the child was born at a GA of 31 weeks, and this is a common pathology in preterm children, this cannot be directly related to the maternal cancer treatment. The second child, a boy born at 38 weeks of gestation, was diagnosed with a focal cortical dysplasia in the left medial parieto-occipital sulcus. This is a pathology of neuronal migration, which most likely is due to a fetal impact at a GA of 3-5 months ${ }^{16}$. However, as the first chemotherapy cycle was administered at a GA of 29 weeks a link between this therapy and the development of the focal cortical dysplasia is unlikely.

In the control group incidental findings were observed in two children. One girl, born at a GA of 36 weeks, presented bilateral parietal and frontal periventricular leukomalacia. A second girl, born at a GA of 39 weeks, had a left temporo-insular subependymal cyst. 
ACKNOWLEDGEMENTS, PERSONAL CONTRIBUTION AND CONFLICT OF INTEREST STATEMENTS 


\section{Scientific acknowledgement}

Parts of this research has been realized through funding from the European Union's Horizon 2020 research and innovation programme (European Research Council, grant no 647047), Foundation against cancer (Stichting tegen kanker, grants no. 2014-152 and no. 2010-201) and Research Foundation Flanders (FWO, grant no. 11B9919N). Prof. Frédéric amant and Prof. Stefan Sunaert have received funding as senior clinical researchers for the FWO. Jeroen Blommaert and Tineke Vandenbroucke have received funding as aspirant researchers for FWO. The computational resources and services used in this work were provided by the VSC (Flemish Supercomputer Center), funded by the FWO and the Flemish Government - department EWI.

The authors would like to thank Jorine De Haan, An-sofie Gorissen, Annouscka Laenen, Liesbeth Leemans, Gunnar Naulaers, Kaat Philippe, Cettina Schellens, Lara Stroobants, Tineke Vandenbroucke, Marie-Astrid Van Hoorick, Katrien Van Tornout, Dorothée Vercruysse, Magali Verheecke and Diane Wolput for their contributions to this work and Isabeau Veelaert for designing the cover art.

\section{Personal contribution}

The $\mathrm{PhD}$ candidate wrote the manuscript and the included publications. He performed all imaging and statistical analyses, with the assistance of the respective co-authors of these chapters. The Correlation analysis in chapter 4 was performed by Gwen Schroyen. The PhD performed data acquisition and management for the studies in chapters 5 and 6 . Part of the data acquisition in chapter 5 was performed by Ivan Myatchin, Tineke Vandenbroucke and Dorothee Vercruysse. Part of the data acquisition in chapter 6 was performed by Dorothee Vercruysse and Charlotte Sleurs. For both chapters however, the majority of data was acquired by the PhD candidate.

\section{Conflict of interest statement}

The $\mathrm{PhD}$ candidate has no conflicts of interest to disclose 


\section{BIBLIOGRAPHY}

[1] K. M. Fancher, V. Giannetti, and B. T. Mclaughlin, "Ethics of chemotherapy during pregnancy," American Journal of Health-System Pharmacy, vol. 76, no. 4, pp. 242-247, 2019.

[2] J. de Haan, M. Verheecke, K. Van Calsteren, B. Van Calster, R. G. Shmakov, M. Mhallem Gziri, M. J. Halaska, R. Fruscio, C. A. R. Lok, I. A. Boere, P. Zola, P. B. Ottevanger, C. J. M. de Groot, F. A. Peccatori, K. Dahl Steffensen, E. H. Cardonick, E. Polushkina, L. Rob, L. Ceppi, G. T. Sukhikh, S. N. Han, and F. Amant, "Oncological management and obstetric and neonatal outcomes for women diagnosed with cancer during pregnancy: a 20-year international cohort study of 1170 patients," The Lancet Oncology, vol. 19, pp. 337-346, mar 2018.

[3] L. Lenaerts, P. Vandenberghe, N. Brison, H. Che, M. Neofytou, M. Verheecke, L. Leemans, C. Maggen, B. Dewaele, L. Dehaspe, S. Vanderschueren, D. Dierickx, V. Vandecaveye, F. Amant, and J. Vermeesch, "Genomewide copy number alteration screening of circulating plasma DNA: potential for the detection of incipient tumors," Annals of Oncology, vol. 30, pp. 85-95, jan 2019.

[4] L. Lenaerts, T. Jatsenko, F. Amant, and J. Robert Vermeesch, "Noninvasive Prenatal Testing and Detection of Occult Maternal Malignancies," Clinical Chemistry, vol. 65, pp. 1484-1486, dec 2019.

[5] F. Amant, K. Van Calsteren, M. J. Halaska, M. M. Gziri, W. Hui, L. Lagae, M. A. Willemsen, L. Kapusta, B. Van Calster, H. Wouters, L. Heyns, S. N. Han, V. Tomek, L. Mertens, and P. B. Ottevanger, "Long-term cognitive and cardiac outcomes after prenatal exposure to chemotherapy in children aged 18 months or older: an observational study," The Lancet Oncology, vol. 13, pp. 256-264, mar 2012.

[6] F. Amant, T. Vandenbroucke, M. Verheecke, M. Fumagalli, M. J. Halaska, I. Boere, S. Han, M. M. Gziri, F. Peccatori, L. Rob, C. Lok, P. Witteveen, J.-U. Voigt, G. Naulaers, L. Vallaeys, 
F. Van den Heuvel, L. Lagae, L. Mertens, L. Claes, K. Van Calsteren, and International Network on Cancer, Infertility, and Pregnancy (INCIP), "Pediatric Outcome after Maternal Cancer Diagnosed during Pregnancy.," The New England journal of medicine, vol. 373, no. 19, pp. 1824-34, 2015.

[7] K. Van Calsteren, L. Heyns, F. De Smet, L. Van Eycken, M. M. Gziri, W. Van Gemert, M. Halaska, I. Vergote, N. Ottevanger, and F. Amant, "Cancer during pregnancy: An analysis of 215 patients emphasizing the obstetrical and the Neonatal outcomes," Journal of Clinical Oncology, vol. 28, no. 4, pp. 683-689, 2010.

[8] K. Van Calsteren, R. Verbesselt, J. Beijnen, R. Devlieger, L. De Catte, D. C. Chai, R. Van Bree, L. Heyns, J. De Hoon, and F. Amant, "Transplacental transfer of anthracyclines, vinblastine, and 4-hydroxy-cyclophosphamide in a baboon model," Gynecologic Oncology, vol. 119, no. 3, pp. 594-600, 2010.

[9] K. Van Calsteren, R. Verbesselt, R. Devlieger, L. De Catte, D. Chai, R. Van Bree, L. Heyns, J. Beijnen, S. Demarsin, E. de Bruijn, J. de Hoon, and F. Amant, "Transplacental Transfer of Paclitaxel, Docetaxel, Carboplatin, and Trastuzumab in a Baboon Model," International Journal of Gynecological Cancer, vol. 20, no. 9, pp. 1459-1464, 2010.

[10] E. H. Cardonick, M. B. Gringlas, K. Hunter, and J. Greenspan, "Development of children born to mothers with cancer during pregnancy: Comparing in utero chemotherapy-exposed children with nonexposed controls," American Journal of Obstetrics and Gynecology, vol. 212, no. 5 , pp. 658.e1-658.e8, 2015.

[11] P. Berveiller, O. Marty, F. Vialard, and O. Mir, "Use of anticancer agents in gynecological oncology during pregnancy: a systematic review of maternal pharmacokinetics and transplacental transfer," Expert Opinion on Drug Metabolism \& Toxicology, vol. 12, pp. 523-531, may 2016.

[12] F. Amant, S. N. Han, M. M. Gziri, T. Vandenbroucke, M. Verheecke, and K. Van Calsteren, "Management of cancer in pregnancy.," Best practice \& research. Clinical obstetrics \& gynaecology, vol. 29, no. 5, pp. 741-753, 2015.

[13] E. Cardonick, E. Eicheldinger, and J. P. Gaughan, "Chemotherapy is avoided during the first trimester of pregnancy, when is the safest time to start treatment during the second or third trimester?," ProClinS Gynecology and Obstetrics, vol. 2, no. 1, p. 1005, 2019.

[14] T. Vandenbroucke, M. Verheecke, M. van Gerwen, K. Van Calsteren, M. J. Halaska, M. Fumagalli, R. Fruscio, A. Gandhi, M. Veening, L. Lagae, P. B. Ottevanger, J.-U. Voigt, J. de Haan, M. M. Gziri, C. Maggen, L. Mertens, G. Naulaers, L. Claes, F. Amant, and on behalf of the International Network on Cancer, Infertility and Pregnancy (INCIP) , "Child development at 6 years after maternal cancer diagnosis and treatment during pregnancy," European Journal of Cancer, vol. 138 , pp. 57-67, oct 2020. 
[15] K. Van Calsteren, D. Hartmann, L. Van Aerschot, R. Verbesselt, R. Van Bree, R. D'Hooge, and F. Amant, "Vinblastine and doxorubicin administration to pregnant mice affects brain development and behaviour in the offspring," NeuroToxicology, vol. 30, pp. 647-657, jul 2009.

[16] A. C. Jansen and K. Keymolen, Fetal and neonatal neurogenetics, vol. 162. Elsevier B.V., 1 ed., 2019.

[17] S. Passera, V. Contarino, G. Scarfone, E. Scola, C. Fontana, F. Peccatori, C. Cinnante, S. Counsell, M. Ossola, S. Pisoni, N. Pesenti, E. Grossi, F. Amant, F. Mosca, F. Triulzi, and M. Fumagalli, "Effects of in-utero exposure to chemotherapy on fetal brain growth," International Journal of Gynecologic Cancer, pp. ijgc-2019-000416, 2019.

[18] C. Sleurs, S. Deprez, L. Emsell, J. Lemiere, and A. Uyttebroeck, "Chemotherapy-induced neurotoxicity in pediatric solid non-CNS tumor patients: An update on current state of research and recommended future directions," Critical Reviews in Oncology/Hematology, vol. 103, pp. 37-48, 2016.

[19] T. M. Brinkman, C. J. Recklitis, G. Michel, M. A. Grootenhuis, and J. L. Klosky, "Psychological Symptoms, Social Outcomes, Socioeconomic Attainment, and Health Behaviors Among Survivors of Childhood Cancer: Current State of the Literature," Journal of Clinical Oncology, vol. 36, pp. 2190-2197, jul 2018.

[20] T. A. Ahles and J. C. Root, "Cognitive Effects of Cancer and Cancer Treatments," Annual Review of Clinical Psychology, vol. 14, pp. 425-451, may 2018.

[21] M. C. Janelsins, S. R. Kesler, T. A. Ahles, and G. R. Morrow, "Prevalence, mechanisms, and management of cancer-related cognitive impairment.," International review of psychiatry (Abingdon, England), vol. 26, pp. 102-13, feb 2014.

[22] B. C. McDonald and A. J. Saykin, "Alterations in brain structure related to breast cancer and its treatment: chemotherapy and other considerations," Brain Imaging and Behavior, vol. 7 , pp. 374-387, dec 2013.

[23] S. Deprez, M. Vandenbulcke, R. Peeters, L. Emsell, A. Smeets, M.-R. Christiaens, F. Amant, and S. Sunaert, "Longitudinal Assessment of Chemotherapy-Induced Alterations in Brain Activation During Multitasking and Its Relation With Cognitive Complaints," Journal of Clinical Oncology, vol. 32, pp. 2031-2038, jul 2014.

[24] B. C. McDonald, S. K. Conroy, D. J. Smith, J. D. West, and A. J. Saykin, "Frontal gray matter reduction after breast cancer chemotherapy and association with executive symptoms: $A$ replication and extension study," Brain, Behavior, and Immunity, vol. 30, pp. S117-S125, mar 2013. 
[25] C. Sleurs, J. Lemiere, A. Radwan, M. Verly, I. Elens, M. Renard, S. Jacobs, S. Sunaert, S. Deprez, and A. Uyttebroeck, "Long-term leukoencephalopathy and neurocognitive functioning in childhood sarcoma patients treated with high-dose intravenous chemotherapy," Pediatric Blood \& Cancer, jul 2019.

[26] G. Schroyen, M. Meylaers, S. Deprez, J. Blommaert, A. Smeets, S. Jacobs, S. Sunaert, C. Sleurs, and A. Uyttebroeck, "Prevalence of leukoencephalopathy and its potential cognitive sequelae in cancer patients," Journal of Chemotherapy, vol. 0, no. 0, pp. 1-17, 2020. PMID: 32799637.

[27] C. Sleurs, J. Lemiere, D. Christiaens, T. Billiet, R. Peeters, S. Sunaert, A. Uyttebroeck, and S. Deprez, "Advanced MR diffusion imaging and chemotherapy-related changes in cerebral white matter microstructure of survivors of childhood bone and soft tissue sarcoma?," Human Brain Mapping, 2018.

[28] S. Deprez, F. Amant, R. Yigit, K. Porke, J. Verhoeven, J. Van den Stock, A. Smeets, M.-R. Christiaens, A. Leemans, W. Van Hecke, J. Vandenberghe, M. Vandenbulcke, and S. Sunaert, "Chemotherapy-induced structural changes in cerebral white matter and its correlation with impaired cognitive functioning in breast cancer patients.," Human brain mapping, vol. 32 , pp. 480-93, mar 2011.

[29] S. Deprez, F. Amant, A. Smeets, R. Peeters, A. Leemans, W. Van Hecke, J. S. Verhoeven, M.-R. Christiaens, J. Vandenberghe, M. Vandenbulcke, and S. Sunaert, "Longitudinal Assessment of Chemotherapy-Induced Structural Changes in Cerebral White Matter and Its Correlation With Impaired Cognitive Functioning," Journal of Clinical Oncology, vol. 30, pp. 274-281, jan 2012.

[30] T. Billiet, L. Emsell, M. Vandenbulcke, R. Peeters, D. Christiaens, A. Leemans, W. van Hecke, A. Smeets, F. Amant, S. Sunaert, and S. Deprez, "Recovery from chemotherapy-induced white matter changes in young breast cancer survivors?," Brain Imaging and Behavior, pp. 1-14, 2017.

[31] T. Billiet, I. Elens, C. Sleurs, A. Uyttebroeck, R. D'Hooge, J. Lemiere, and S. Deprez, "Brain Connectivity and Cognitive Flexibility in Nonirradiated Adult Survivors of Childhood Leukemia," JNCI: Journal of the National Cancer Institute, vol. 110, pp. 905-913, aug 2018.

[32] B. C. McDonald, S. K. Conroy, T. A. Ahles, J. D. West, and A. J. Saykin, "Gray matter reduction associated with systemic chemotherapy for breast cancer: a prospective MRI study," Breast Cancer Research and Treatment, vol. 123, pp. 819-828, oct 2010.

[33] J. S. Mandelblatt, A. Hurria, B. C. McDonald, A. J. Saykin, R. A. Stern, J. W. VanMeter, M. McGuckin, T. Traina, N. Denduluri, S. Turner, D. Howard, P. B. Jacobsen, and T. Ahles, "Cognitive Effects of Cancer and Its Treatments at the Intersection of Aging: What Do We Know; What Do We Need to Know?," Seminars in Oncology, vol. 40, pp. 709-725, dec 2013.

[34] M. B. De Ruiter, L. Reneman, W. Boogerd, D. J. Veltman, M. Caan, G. Douaud, C. Lavini, S. C. Linn, E. Boven, F. S. A. M. Van Dam, and S. B. Schagen, "Late effects of high-dose adjuvant 
chemotherapy on white and gray matter in breast cancer survivors: Converging results from multimodal magnetic resonance imaging," Human Brain Mapping, vol. 33, pp. 2971-2983, dec 2012 .

[35] V. Koppelmans, M. B. de Ruiter, F. van der Lijn, W. Boogerd, C. Seynaeve, A. van der Lugt, H. Vrooman, W. J. Niessen, M. M. B. Breteler, and S. B. Schagen, "Global and focal brain volume in long-term breast cancer survivors exposed to adjuvant chemotherapy," Breast Cancer Research and Treatment, vol. 132, pp. 1099-1106, apr 2012.

[36] C. Sleurs, J. Blommaert, D. Batalle, M. Verly, S. Sunaert, R. Peeters, J. Lemiere, A. Uyttebroeck, and S. Deprez, "Cortical thinning and altered functional brain coherence in survivors of childhood sarcoma," Brain Imaging and Behavior, apr 2020.

[37] T. A. Ahles and A. J. Saykin, "Candidate mechanisms for chemotherapy-induced cognitive changes," Nature Reviews Cancer, vol. 7, pp. 192-201, mar 2007.

[38] J. S. Wefel, S. R. Kesler, K. R. Noll, and S. B. Schagen, "Clinical characteristics, pathophysiology, and management of noncentral nervous system cancer-related cognitive impairment in adults," CA: A Cancer Journal for Clinicians, vol. 65, pp. 123-138, mar 2015.

[39] R. S. Bourke, C. R. West, G. Chheda, and D. B. Tower, "Kinetics of entry and distribution of 5-fluorouracil in cerebrospinal fluid and brain following intravenous injection in a primate.," Cancer research, vol. 33, pp. 1735-46, jul 1973.

[40] F. E. Froklage, J. C. Reijneveld, and J. J. Heimans, "Central neurotoxicity in cancer chemotherapy: pharmacogenetic insights," Pharmacogenomics, vol. 12, pp. 379-395, mar 2011.

[41] S. Sugimoto, Y. L. Yamamoto, S. Nagahiro, and M. Diksic, "Permeability change and brain tissue damage after intracarotid administration of cisplatin studied by double-tracer autoradiography in rats," Journal of Neuro-Oncology, vol. 24, no. 3, pp. 229-240, 1995.

[42] R. Seigers, M. Loos, O. V. Tellingen, W. Boogerd, A. B. Smit, and S. B. Schagen, "Neurobiological changes by cytotoxic agents in mice," Behavioural Brain Research, vol. 299, pp. 19-26, 2016.

[43] N. M. Mounier, A. E.-S. Abdel-Maged, S. A. Wahdan, A. M. Gad, and S. S. Azab, "Chemotherapyinduced cognitive impairment $(\mathrm{ClCl})$ : An overview of etiology and pathogenesis," Life Sciences, vol. 258, p. 118071 , oct 2020 .

[44] D. C.-M. Vercruysse, S. Deprez, S. Sunaert, K. Van Calsteren, and F. Amant, "Effects of prenatal exposure to cancer treatment on neurocognitive development, a review," NeuroToxicology, vol. 54, pp. 11-21, may 2016.

[45] M. S. Scher, "Fetal neurology: Principles and practice with a life-course perspective," in Handbook of Clinical Neurology, vol. 162, pp. 1-29, Elsevier B.V., 1 ed., 2019. 
[46] S. J. Counsell, T. Arichi, S. Arulkumaran, and M. A. Rutherford, Fetal and neonatal neuroimaging, vol. 162. Elsevier B.V., 1 ed., 2019.

[47] S. E. Cusick and M. K. Georgieff, "The Role of Nutrition in Brain Development: The Golden Opportunity of the "First 1000 Days"," Journal of Pediatrics, vol. 175, pp. 16-21, 2016.

[48] W. M. Cowan, "The development of the brain," Scientific American, vol. 241, no. 3, pp. 112-133, 1979.

[49] T. White, S. Su, M. Schmidt, C.-Y. Kao, and G. Sapiro, "The development of gyrification in childhood and adolescence," Brain and Cognition, vol. 72, pp. 36-45, feb 2010.

[50] E. Luders, P. M. Thompson, and A. W. Toga, "The development of the corpus callosum in the healthy human brain," Journal of Neuroscience, vol. 30, no. 33, pp. 10985-10990, 2010.

[51] C. K. Tamnes, D. R. Roalf, A.-I. L. Goddings, and C. Lebel, "Diffusion MRI of white matter microstructure development in childhood and adolescence : Methods, challenges and progress," Developmental Cognitive Neuroscience, vol. 190, no. max 200, 2017.

[52] S. Genc, M. L. Seal, T. Dhollander, C. B. Malpas, P. Hazell, and T. J. Silk, "White matter alterations at pubertal onset," Neurolmage, vol. 156, no. May, pp. 286-292, 2017.

[53] S. Genc, C. B. Malpas, G. Ball, T. J. Silk, and M. L. Seal, "Age, sex, and puberty related development of the corpus callosum: a multi-technique diffusion MRI study," Brain Structure and Function, vol. 223, no. 6, pp. 1-13, 2018.

[54] S. Genc, R. E. Smith, C. B. Malpas, V. Anderson, J. M. Nicholson, D. Efron, E. Sciberras, M. L. Seal, and T. J. Silk, "Development of white matter fibre density and morphology over childhood: A longitudinal fixel-based analysis," Neurolmage, vol. 183, pp. 666-676, dec 2018.

[55] C. K. Tamnes, M. M. Herting, A.-L. Goddings, R. Meuwese, S.-J. Blakemore, R. E. Dahl, B. Güroğlu, A. Raznahan, E. R. Sowell, E. A. Crone, and K. L. Mills, "Development of the Cerebral Cortex across Adolescence: A Multisample Study of Inter-Related Longitudinal Changes in Cortical Volume, Surface Area, and Thickness," The Journal of Neuroscience, vol. 37, pp. 3402-3412, mar 2017.

[56] S. Ducharme, M. D. Albaugh, T.-V. Nguyen, J. J. Hudziak, J. Mateos-Pérez, A. Labbe, A. C. Evans, and S. Karama, "Trajectories of cortical thickness maturation in normal brain development - The importance of quality control procedures," Neurolmage, vol. 125, pp. 267-279, jan 2016.

[57] Y. Aleman-Gomez, J. Janssen, H. Schnack, E. Balaban, L. Pina-Camacho, F. Alfaro-Almagro, J. Castro-Fornieles, S. Otero, I. Baeza, D. Moreno, N. Bargallo, M. Parellada, C. Arango, and M. Desco, "The Human Cerebral Cortex Flattens during Adolescence," Journal of Neuroscience, vol. 33, pp. 15004-15010, sep 2013. 
[58] E. Derbyshire and R. Obeid, "Choline, neurological development and brain function: A systematic review focusing on the first 1000 days," Nutrients, vol. 12, no. 6, pp. 1-32, 2020.

[59] P. R. Britto, S. J. Lye, K. Proulx, A. K. Yousafzai, S. G. Matthews, T. Vaivada, R. Perez-Escamilla, N. Rao, P. Ip, L. C. Fernald, H. MacMillan, M. Hanson, T. D. Wachs, H. Yao, H. Yoshikawa, A. Cerezo, J. F. Leckman, and Z. A. Bhutta, "Nurturing care: promoting early childhood development," The Lancet, vol. 389, no. 10064, pp. 91-102, 2017.

[60] I. Novak and C. Morgan, High-risk follow-up: Early intervention and rehabilitation, vol. 162. Elsevier B.V., 1 ed., 2019.

[61] J. Karbach and K. Unger, "Executive control training from middle childhood to adolescence.," Frontiers in psychology, vol. 5, no. MAY, p. 390, 2014.

[62] M. K. Holder and J. D. Blaustein, "Puberty and adolescence as a time of vulnerability to stressors that alter neurobehavioral processes," Frontiers in Neuroendocrinology, vol. 35, pp. 89-110, jan 2014.

[63] B. R. H. van den Bergh, R. Dahnke, and M. Mennes, "Prenatal stress and the developing brain: Risks for neurodevelopmental disorders," Development and Psychopathology, vol. 30, pp. 743-762, aug 2018.

[64] S. N. Mattson, G. A. Bernes, and L. R. Doyle, "Fetal Alcohol Spectrum Disorders: A Review of the Neurobehavioral Deficits Associated With Prenatal Alcohol Exposure," Alcoholism: Clinical and Experimental Research, p. acer.14040, may 2019.

[65] K. B. Walhovd, V. Moe, K. Slinning, P. Due-Tønnessen, A. Bjørnerud, A. M. Dale, A. van der Kouwe, B. T. Quinn, B. Kosofsky, D. Greve, and B. Fischl, "Volumetric cerebral characteristics of children exposed to opiates and other substances in utero," Neurolmage, vol. 36, no. 4, pp. 1331-1344, 2007.

[66] O. Boucher, M. J. Burden, G. Muckle, D. Saint-Amour, P. Ayotte, É. Dewailly, C. A. Nelson, S. W. Jacobson, and J. L. Jacobson, "Response inhibition and error monitoring during a visual Go/No-Go task in Inuit children exposed to lead, polychlorinated biphenyls, and methylmercury," Environmental Health Perspectives, vol. 120, no. 4, pp. 608-615, 2012.

[67] J. G. Dórea, "Environmental exposure to low-level lead (Pb) co-occurring with other neurotoxicants in early life and neurodevelopment of children.," Environmental Research, vol. 177, p. 108641, oct 2019.

[68] O. Boucher, J. L. Jacobson, M. J. Burden, E. Dewailly, S. W. Jacobson, G. Muckle, É. Dewailly, S. W. Jacobson, and G. Muckle, "Prenatal tobacco exposure and response inhibition in school-aged children: an event-related potential study.," Neurotoxicology and teratology, vol. 44, p. 81 , jul 2014. 
[69] K. Räikkönen, S. Martikainen, A. K. Pesonen, J. Lahti, K. Heinonen, R. Pyhälä, M. Lahti, S. Tuovinen, K. Wehkalampi, S. Sammallahti, L. Kuula, S. Andersson, J. G. Eriksson, A. Ortega-Alonso, R. M. Reynolds, T. E. Strandberg, J. R. Seckl, and E. Kajantie, "Maternal licorice consumption during pregnancy and pubertal, cognitive, and psychiatric outcomes in children," American Journal of Epidemiology, vol. 185, no. 5, pp. 317-328, 2017.

[70] A. J. Degnan, J. L. Wisnowski, S. Choi, R. Ceschin, C. Bhushan, R. M. Leahy, P. Corby, V. J. Schmithorst, and A. Panigrahy, "Altered structural and functional connectivity in late preterm preadolescence: An anatomic seed-based study of resting state networks related to the posteromedial and lateral parietal cortex," PLoS ONE, vol. 10, no. 6, pp. 1-22, 2015.

[71] A. J. Degnan, J. L. Wisnowski, S. Choi, R. Ceschin, C. Bhushan, R. M. Leahy, P. Corby, V. J. Schmithorst, and A. Panigrahy, "Alterations of resting state networks and structural connectivity in relation to the prefrontal and anterior cingulate cortices in late prematurity.," Neuroreport, vol. 26, pp. 22-6, jan 2015 .

[72] C. A. van Houdt, J. Oosterlaan, A. G. Wassenaer-Leemhuis, A. H. Kaam, and C. S. AarnoudseMoens, "Executive function deficits in children born preterm or at low birthweight: a meta-analysis," Developmental Medicine \& Child Neurology, p. dmcn.14213, apr 2019.

[73] K. B. Walhovd, A. M. Fjell, T. T. Brown, J. M. Kuperman, Y. Chung, D. J. Hagler, J. C. Roddey, M. Erhart, C. McCabe, N. Akshoomoff, D. G. Amaral, C. S. Bloss, O. Libiger, N. J. Schork, B. F. Darst, B. J. Casey, L. Chang, T. M. Ernst, J. Frazier, J. R. Gruen, W. E. Kaufmann, S. S. Murray, P. Van Zijl, S. Mostofsky, and A. M. Dale, "Long-term influence of normal variation in neonatal characteristics on human brain development," Proceedings of the National Academy of Sciences of the United States of America, vol. 109, no. 49, pp. 20089-20094, 2012.

[74] S. Suffren, D. Angulo, Y. Ding, P. Reyes, J. Marin, J. T. Hernandez, N. Charpak, and G. A. Lodygensky, "Long-term attention deficits combined with subcortical and cortical structural central nervous system alterations in young adults born small for gestational age," Early Human Development, vol. 110, pp. 44-49, jul 2017.

[75] B. R. Van den Bergh, M. I. van den Heuvel, M. Lahti, M. Braeken, S. R. de Rooij, S. Entringer, D. Hoyer, T. Roseboom, K. Räikkönen, S. King, and M. Schwab, "Prenatal developmental origins of behavior and mental health: The influence of maternal stress in pregnancy," Neuroscience \& Biobehavioral Reviews, jul 2017.

[76] A. M. Graham, J. M. Rasmussen, S. Entringer, E. Ben Ward, M. D. Rudolph, J. H. Gilmore, M. Styner, P. D. Wadhwa, D. A. Fair, and C. Buss, "Maternal Cortisol Concentrations During Pregnancy and Sex-Specific Associations With Neonatal Amygdala Connectivity and Emerging Internalizing Behaviors," Biological Psychiatry, vol. 85, pp. 172-181, jan 2019. 
[77] C. Buss, E. P. Davis, L. T. Muftuler, K. Head, and C. A. Sandman, "High pregnancy anxiety during mid-gestation is associated with decreased gray matter density in 6-9-year-old children," Psychoneuroendocrinology, vol. 35, no. 1, pp. 141-153, 2010.

[78] H. El Marroun, R. Zou, R. L. Muetzel, V. W. Jaddoe, F. C. Verhulst, T. White, and H. Tiemeier, "Prenatal exposure to maternal and paternal depressive symptoms and white matter microstructure in children," Depression and Anxiety, vol. 35, no. 4, pp. 321-329, 2018.

[79] H. El Marroun, H. Tiemeier, R. L. Muetzel, S. Thijssen, N. J. van der Knaap, V. W. Jaddoe, G. Fernández, F. C. Verhulst, and T. J. White, "Prenatal Exposure To Maternal and Paternal Depressive Symptoms and Brain Morphology: a Population-Based Prospective Neuroimaging Study in Young Children," Depression and Anxiety, vol. 33, no. 7, pp. 658-666, 2016.

[80] S. R. Veena, C. R. Gale, G. V. Krishnaveni, S. H. Kehoe, K. Srinivasan, and C. H. Fall, "Association between maternal nutritional status in pregnancy and offspring cognitive function during childhood and adolescence; a systematic review," BMC Pregnancy and Childbirth, vol. 16, no. 1,2016 .

[81] G. E. Hodes and C. N. Epperson, "Sex Differences in Vulnerability and Resilience to Stress Across the Life Span," Biological Psychiatry, vol. 86, no. 6, pp. 421-432, 2019.

[82] M. Betchen, V. A. Grunberg, M. Gringlas, and E. Cardonick, "Being a mother after a cancer diagnosis during pregnancy: Maternal psychosocial functioning and child cognitive development and behavior," Psycho-Oncology, no. March, pp. 1-8, 2020.

[83] S. Genc, C. B. Malpas, A. Gulenc, E. Sciberras, D. Efron, T. J. Silk, and M. L. Seal, "Longitudinal white matter development in children is associated with puberty, attentional difficulties, and mental health .," bioRxiv, 2019.

[84] M. C. Stevens, "The contributions of resting state and task-based functional connectivity studies to our understanding of adolescent brain network maturation," Neuroscience and Biobehavioral Reviews, vol. 70, pp. 13-32, 2016.

[85] M. Downes, J. Bathelt, and M. De Haan, "Event-related potential measures of executive functioning from preschool to adolescence," Developmental Medicine \& Child Neurology, vol. 59, pp. 581-590, jun 2017.

[86] A. Jack, "Neuroimaging in neurodevelopmental disorders: Focus on resting-state fMRI analysis of intrinsic functional brain connectivity," Current Opinion in Neurology, vol. 31, no. 2, pp. 140-148, 2018.

[87] D. N. Greve, "An Absolute Beginner's Guide to Surface- and Voxel-based Morphometric Analysis," Proceedings of the International Society for Magnetic Resonance in Medicine, vol. i, pp. 1-7, 2011. 
[88] J. Ashburner and K. J. Friston, "Voxel-Based Morphometry-The Methods," Neurolmage, vol. 11, no. 6, pp. 805-821, 2000.

[89] A. Mechelli, C. Price, K. Friston, and J. Ashburner, "Voxel-Based Morphometry of the Human Brain: Methods and Applications," Current Medical Imaging Reviews, vol. 1, pp. 105-113, jun 2005.

[90] V. Baliyan, C. J. Das, R. Sharma, and A. K. Gupta, "Diffusion weighted imaging: Technique and applications," World Journal of Radiology, vol. 8, no. 9, p. 785, 2016.

[91] W. Van Hecke, L. Emsell, and S. Sunaert, eds., Diffusion Tensor Imaging. New York, NY: Springer New York, 2016.

[92] M. P. van den Heuvel and H. E. Hulshoff Pol, "Exploring the brain network: A review on resting-state fMRI functional connectivity," European Neuropsychopharmacology, vol. 20, no. 8, pp. 519-534, 2010.

[93] F. Barkhof, S. Haller, and S. a. R. B. Rombouts, "Resting-State Functional MR Imaging: A New Window to the Brain.," Radiology, vol. 272, no. 1, pp. 29-49, 2014.

[94] S. J. Luck, An Introduction to the Event-Related Potential Technique. MIT Press, 2 ed., 2014.

[95] S. Nieuwenhuis, N. Yeung, W. van den Wildenberg, and K. R. Ridderinkhof, "Electrophysiological correlates of anterior cingulate function in a go/no-go task: effects of response conflict and trial type frequency.," Cognitive, Affective \& Behavioral Neuroscience, vol. 3, pp. 17-26, mar 2003.

[96] J. Harper, S. M. Maloneb, E. M. Bernat, S. M. Malone, and E. M. Bernat, "Theta and delta band activity explain N2 and P3 ERP component activity in a go/no-go task," Clin Neurophysiol., vol. 125, pp. 124-132, jan 2014.

[97] F. C. L. Donkers and G. J. M. Van Boxtel, "The N2 in go/no-go tasks reflects conflict monitoring not response inhibition," Brain and Cognition, vol. 56, no. 2 SPEC. ISS., pp. 165-176, 2004.

[98] M. I. Posner, "Orienting of attention," Quarterly Journal of Experimental Psychology, vol. 32:1, no. May, pp. 3-25, 1980.

[99] M. Eimer and J. Driver, "Crossmodal links in endogenous and exogenous spatial attention: evidence from event-related brain potential studies.," Neuroscience and biobehavioral reviews, vol. 25, no. 6, pp. 497-511, 2001.

[100] C. Perchet and L. Garcia-Larrea, "Visuospatial attention and motor reaction in children: An electrophysiological study of the "Posner" paradigm," Psychophysiology, vol. 37, pp. 231-241, mar 2000. 
[101] H. A. Slagter, A. Kok, N. Mol, D. Talsma, and J. L. Kenemans, "Generating spatial and nonspatial attentional control: An ERP study," Psychophysiology, vol. 42, no. 4, pp. 428-439, 2005.

[102] I. L. Pykett, J. H. Newhouse, F. S. Buonanno, T. J. Brady, M. R. Goldman, J. P. Kistler, and G. M. Pohost, "Principles of nuclear magnetic resonance imaging," Radiology, vol. 143, no. 1, pp. 157-168, 1982.

[103] S. J. Kim and K. A. Kim, "Safety issues and updates under MR environments.," European journal of radiology, vol. 89, pp. 7-13, apr 2017.

[104] G. L. Baum, D. R. Roalf, P. A. Cook, R. Ciric, A. F. Rosen, C. Xia, M. A. Elliott, K. Ruparel, R. Verma, B. Tunç, R. C. Gur, R. E. Gur, D. S. Bassett, and T. D. Satterthwaite, "The impact of in-scanner head motion on structural connectivity derived from diffusion MRI," Neurolmage, vol. 173, no. February, pp. 275-286, 2018.

[105] A. Gilmore, N. Buser, and J. L. Hanson, "Variations in Structural MRI Quality Impact Measures of Brain Anatomy: Relations with Age and Other Sociodemographic Variables," bioRxiv, 2019.

[106] A. Klein, S. S. Ghosh, F. S. Bao, J. Giard, Y. Häme, E. Stavsky, N. Lee, B. Rossa, M. Reuter, E. Chaibub Neto, and A. Keshavan, Mindboggling morphometry of human brains, vol. 13. 2017.

[107] R. H. R. Pruim, M. Mennes, D. van Rooij, A. Llera, J. K. Buitelaar, and C. F. Beckmann, "ICA-AROMA: A robust ICA-based strategy for removing motion artifacts from fMRI data.," Neurolmage, vol. 112, pp. 267-277, may 2015.

[108] O. Esteban, C. J. Markiewicz, R. W. Blair, C. A. Moodie, A. I. Isik, A. Erramuzpe, J. D. Kent, M. Goncalves, E. DuPre, M. Snyder, H. Oya, S. S. Ghosh, J. Wright, J. Durnez, R. A. Poldrack, and K. J. Gorgolewski, "fMRIPrep: a robust preprocessing pipeline for functional MRI," Nature Methods, vol. 16, pp. 111-116, jan 2019.

[109] D. Christiaens, P. J. Slator, L. Cordero-Grande, A. N. Price, M. Deprez, D. C. Alexander, M. Rutherford, J. V. Hajnal, and J. Hutter, "In utero diffusion MRI: challenges, advances, and applications," no. June, 2019.

[110] M. Jenkinson, P. Bannister, M. Brady, and S. Smith, "Improved Optimization for the Robust and Accurate Linear Registration and Motion Correction of Brain Images," Neurolmage, vol. 17, pp. 825-841, oct 2002.

[111] R. W. Cox, "AFNI: Software for Analysis and Visualization of Functional Magnetic Resonance Neuroimages," Computers and Biomedical Research, vol. 29, pp. 162-173, jun 1996.

[112] O. Esteban, D. Birman, M. Schaer, O. O. Koyejo, R. A. Poldrack, and K. J. Gorgolewski, "MRIQC: Advancing the automatic prediction of image quality in MRI from unseen sites," PLOS ONE, vol. 12, p. e0184661, sep 2017. 
[113] P. M. Parés-Casanova, "Introductory Chapter - Morphometric Studies: Beyond Pure Anatomical Form Analysis," in New Insights into Morphometry Studies, InTech, jul 2017.

[114] B. Cao, B. Mwangi, I. C. Passos, M.-J. Wu, Z. Keser, G. B. Zunta-Soares, D. Xu, K. M. Hasan, and J. C. Soares, "Lifespan Gyrification Trajectories of Human Brain in Healthy Individuals and Patients with Major Psychiatric Disorders," Scientific Reports, vol. 7, no. 1, p. 511, 2017.

[115] J. Ashburner, C. Hutton, R. Frackowiak, I. Johnsrude, C. Price, and K. Friston, "Identifying global anatomical differences: deformation-based morphometry.," Human brain mapping, vol. 6, no. 5-6, pp. 348-57, 1998.

[116] B. Fischl and A. M. Dale, "Measuring the thickness of the human cerebral cortex from magnetic resonance images," Proceedings of the National Academy of Sciences, vol. 97, pp. 11050-11055, sep 2000.

[117] J. E. Chen and G. H. Glover, "Functional Magnetic Resonance Imaging Methods," Neuropsychology Review, vol. 25, no. 3, pp. 289-313, 2015.

[118] C. Caballero-Gaudes and R. C. Reynolds, "Methods for cleaning the BOLD fMRI signal," Neurolmage, vol. 154, pp. 128-149, jul 2017.

[119] S. Deprez, M. Vandenbulcke, R. Peeters, L. Emsell, F. Amant, and S. Sunaert, "The functional neuroanatomy of multitasking: Combining dual tasking with a short term memory task," Neuropsychologia, vol. 51, pp. 2251-2260, sep 2013.

[120] M. D. Fox and M. D. Greicius, "Clinical applications of resting state functional connectivity," Frontiers in systems neuroscience, vol. 4, no. June, p. 19, 2010.

[121] J. M. Soares, P. Marques, V. Alves, and N. Sousa, "A hitchhiker's guide to diffusion tensor imaging," Frontiers in Neuroscience, vol. 7, no. 7 MAR, pp. 1-14, 2013.

[122] B. Jeurissen, A. Leemans, J.-D. Tournier, D. K. Jones, and J. Sijbers, “Investigating the prevalence of complex fiber configurations in white matter tissue with diffusion magnetic resonance imaging," Human Brain Mapping, vol. 34, no. 11, pp. 2747-2766, 2013.

[123] H. Zhang, T. Schneider, C. A. Wheeler-Kingshott, and D. C. Alexander, "NODDI: Practical in vivo neurite orientation dispersion and density imaging of the human brain," Neurolmage, vol. 61, no. 4, pp. 1000-1016, 2012.

[124] D. Raffelt, J.-D. Tournier, S. Rose, G. R. Ridgway, R. Henderson, S. Crozier, O. Salvado, and A. Connelly, "Apparent Fibre Density: a novel measure for the analysis of diffusion-weighted magnetic resonance images.," Neurolmage, vol. 59, pp. 3976-94, feb 2012.

[125] D. A. Raffelt, J. D. Tournier, R. E. Smith, D. N. Vaughan, G. Jackson, G. R. Ridgway, and A. Connelly, "Investigating white matter fibre density and morphology using fixel-based analysis," Neurolmage, vol. 144, pp. 58-73, 2017. 
[126] B. Jeurissen, J.-D. Tournier, T. Dhollander, A. Connelly, and J. Sijbers, "Multi-tissue constrained spherical deconvolution for improved analysis of multi-shell diffusion MRI data.," Neurolmage, vol. 103, pp. 411-26, dec 2014.

[127] F. A. Azevedo, L. R. Carvalho, L. T. Grinberg, J. M. Farfel, R. E. Ferretti, R. E. Leite, W. J. Filho, R. Lent, and S. Herculano-Houzel, "Equal numbers of neuronal and nonneuronal cells make the human brain an isometrically scaled-up primate brain," The Journal of Comparative Neurology, vol. 513, pp. 532-541, apr 2009.

[128] J. Bock, T. Wainstock, K. Braun, and M. Segal, "Stress In Utero: Prenatal Programming of Brain Plasticity and Cognition," Biological Psychiatry, vol. 78, no. 5, pp. 315-326, 2015.

[129] B. Fischl, "FreeSurfer," Neurolmage, vol. 62, pp. 774-781, aug 2012.

[130] D. Christiaens, M. Reisert, T. Dhollander, S. Sunaert, P. Suetens, and F. Maes, "Global tractography of multi-shell diffusion-weighted imaging data using a multi-tissue model," Neurolmage, vol. 123, pp. 89-101, 2015.

[131] R. E. Smith, J. D. Tournier, F. Calamante, and A. Connelly, "The effects of SIFT on the reproducibility and biological accuracy of the structural connectome," Neurolmage, vol. 104, pp. 253-265, 2015.

[132] D. Batalle, E. J. Hughes, H. Zhang, J.-D. Tournier, N. Tusor, P. Aljabar, L. Wali, D. C. Alexander, J. V. Hajnal, C. Nosarti, A. D. Edwards, and S. J. Counsell, "Early development of structural networks and the impact of prematurity on brain connectivity," Neurolmage, vol. 149, pp. 379-392, apr 2017.

[133] F. D. V. Fallani, L. d. F. Costa, F. A. Rodriguez, L. Astolfi, G. Vecchiato, J. Toppi, G. Borghini, F. Cincotti, D. Mattia, S. Salinari, R. Isabella, and F. Babiloni, "A graph-theoretical approach in brain functional networks. Possible implications in EEG studies," Nonlinear Biomedical Physics, vol. 4, p. S8, jun 2010.

[134] J. Seidlitz, F. Váša, M. Shinn, R. Romero-Garcia, K. J. Whitaker, P. E. Vértes, K. Wagstyl, P. Kirkpatrick Reardon, L. Clasen, S. Liu, A. Messinger, D. A. Leopold, P. Fonagy, R. J. Dolan, P. B. Jones, I. M. Goodyer, A. Raznahan, and E. T. Bullmore, "Morphometric Similarity Networks Detect Microscale Cortical Organization and Predict Inter-Individual Cognitive Variation," Neuron, vol. 97, no. 1, pp. 231-247.e7, 2018.

[135] L. A. Torre, F. Bray, R. L. Siegel, J. Ferlay, J. Lortet-tieulent, and A. Jemal, "Global Cancer Statistics, 2012," CA: a cancer journal of clinicians., vol. 65, no. 2, pp. 87-108, 2015.

[136] H. G. Kaplan, J. A. Malmgren, M. K. Atwood, and G. S. Calip, "Effect of treatment and mammography detection on breast cancer survival over time: 1990-2007.," Cancer, vol. 121, pp. 2553-61, aug 2015. 
[137] R. Han, Y. M. Yang, J. Dietrich, A. Luebke, M. Mayer-Pröschel, and M. Noble, "Systemic 5-fluorouracil treatment causes a syndrome of delayed myelin destruction in the central nervous system.," Journal of biology, vol. 7, no. 4, p. 12, 2008.

[138] C. E. DeSantis, S. A. Fedewa, A. Goding Sauer, J. L. Kramer, R. A. Smith, and A. Jemal, "Breast cancer statistics, 2015: Convergence of incidence rates between black and white women.," CA: a cancer journal for clinicians, vol. 66, pp. 31-42, jan 2016.

[139] M. K. Healey, K. L. Campbell, and L. Hasher, "Chapter 22 Cognitive aging and increased distractibility: Costs and potential benefits," in Progress in Brain Research (W. S. Sossin, J.-C. Lacaille, V. F. Castelucci, and S. Belleville, eds.), vol. 169, pp. 353-363, Elsevier B.V., 2008.

[140] T. A. Ahles, A. J. Saykin, B. C. McDonald, Y. Li, C. T. Furstenberg, B. S. Hanscom, T. J. Mulrooney, G. N. Schwartz, and P. A. Kaufman, "Longitudinal assessment of cognitive changes associated with adjuvant treatment for breast cancer: impact of age and cognitive reserve.," Journal of clinical oncology : official journal of the American Society of Clinical Oncology, vol. 28, pp. 4434-40, oct 2010.

[141] C. M. Schilder, C. Seynaeve, L. V. Beex, W. Boogerd, S. C. Linn, C. M. Gundy, H. M. Huizenga, J. W. Nortier, C. J. van de Velde, F. S. van Dam, and S. B. Schagen, "Effects of tamoxifen and exemestane on cognitive functioning of postmenopausal patients with breast cancer: results from the neuropsychological side study of the tamoxifen and exemestane adjuvant multinational trial.," Journal of clinical oncology : official journal of the American Society of Clinical Oncology, vol. 28, pp. 1294-300, mar 2010.

[142] Q.-L. Xue, "The Frailty Syndrome: Definition and Natural History," Clinics in Geriatric Medicine, vol. 27, pp. 1-15, feb 2011.

[143] M. Lange, O. Rigal, B. Clarisse, B. Giffard, E. Sevin, M. Barillet, F. Eustache, and F. Joly, "Cognitive dysfunctions in elderly cancer patients: A new challenge for oncologists," Cancer Treatment Reviews, vol. 40, no. 6, pp. 810-817, 2014.

[144] C. Lepage, A. M. Smith, J. Moreau, E. Barlow-Krelina, N. Wallis, B. Collins, J. MacKenzie, and C. Scherling, "A prospective study of grey matter and cognitive function alterations in chemotherapy-treated breast cancer patients.," SpringerPlus, vol. 3, no. 1, p. 444, 2014.

[145] M. Inagaki, E. Yoshikawa, Y. Matsuoka, Y. Sugawara, T. Nakano, T. Akechi, N. Wada, S. Imoto, K. Murakami, and Y. Uchitomi, "Smaller regional volumes of brain gray and white matter demonstrated in breast cancer survivors exposed to adjuvant chemotherapy.," Cancer, vol. 109, pp. 146-56, jan 2007.

[146] F. Kurth, E. Luders, and C. Gaser, "Voxel-Based Morphometry," in Brain Mapping, vol. 1, pp. 345-349, Elsevier, 2015. 
[147] C. Gaser, I. Nenadic, B. R. Buchsbaum, E. A. Hazlett, and M. S. Buchsbaum, "Deformation-Based Morphometry and Its Relation to Conventional Volumetry of Brain Lateral Ventricles in MRI," Neurolmage, vol. 13, pp. 1140-1145, jun 2001.

[148] I. Despotović, B. Goossens, and W. Philips, "MRI Segmentation of the Human Brain: Challenges, Methods, and Applications," Computational and Mathematical Methods in Medicine, vol. 2015, pp. 1-23, 2015.

[149] D. E. Broadbent, P. F. Cooper, P. FitzGerald, and K. R. Parkes, "The Cognitive Failures Questionnaire (CFQ) and its correlates," British Journal of Clinical Psychology, vol. 21, pp. 1-16, feb 1982.

[150] C. D. Spielberger, "Assessment of state and trait anxiety: conceptual and methodological issues.," Southern Psychology, vol. 2, pp. 6-16, 1985.

[151] R. J. Bosscher, H. Koning, and R. Van Meurs, "Reliability and Validity of the Beck Depression Inventory in a Dutch College Population," Psychological Reports, vol. 58, pp. 696-698, jun 1986.

[152] B. Schmand, D. Bakker, R. Saan, and J. Louman, "[The Dutch Reading Test for Adults: a measure of premorbid intelligence level].," Tijdschrift voor gerontologie en geriatrie, vol. 22, no. 1, pp. 15-9, 1991.

[153] N. J. Tustison, B. B. Avants, P. A. Cook, Yuanjie Zheng, A. Egan, P. A. Yushkevich, and J. C. Gee, "N4ITK: Improved N3 Bias Correction," IEEE Transactions on Medical Imaging, vol. 29, pp. 1310-1320, jun 2010.

[154] C. Gaser and R. Dahnke, "CAT - A Computational Anatomy Toolbox for the Analysis of Structural MRI Data," in HBM Conference 2012, 2012.

[155] J. Ashburner and G. R. Ridgway, "Symmetric diffeomorphic modeling of longitudinal structural MRI," Frontiers in Neuroscience, vol. 6, no. FEB, pp. 1-19, 2013.

[156] S. Mori, K. Oishi, H. Jiang, L. Jiang, X. Li, K. Akhter, K. Hua, A. V. Faria, A. Mahmood, R. Woods, A. W. Toga, G. B. Pike, P. R. Neto, A. Evans, J. Zhang, H. Huang, M. I. Miller, P. van Zijl, and J. Mazziotta, "Stereotaxic white matter atlas based on diffusion tensor imaging in an ICBM template," Neurolmage, vol. 40, pp. 570-582, apr 2008.

[157] J. C. Pendergrass, S. D. Targum, and J. E. Harrison, "Cognitive Impairment Associated with Cancer: A Brief Review.," Innovations in clinical neuroscience, vol. 15, pp. 36-44, feb 2018.

[158] R. E. Blakesley, S. Mazumdar, M. A. Dew, P. R. Houck, G. Tang, C. F. Reynolds, and M. A. Butters, "Comparisons of methods for multiple hypothesis testing in neuropsychological research.," Neuropsychology, vol. 23, no. 2, pp. 255-264, 2009.

[159] K. Friston, A. Holmes, and K. Worsley, "Statistical parametric maps in functional imaging: a general linear approach," Human Brain Mapping, no. 081, 1994. 
[160] M. A. McDaniel, "Big-brained people are smarter: A meta-analysis of the relationship between in vivo brain volume and intelligence," Intelligence, vol. 33, pp. 337-346, jul 2005.

[161] C. A. Webb, M. Weber, E. A. Mundy, and W. D. S. Killgore, "Reduced gray matter volume in the anterior cingulate, orbitofrontal cortex and thalamus as a function of mild depressive symptoms: a voxel-based morphometric analysis.," Psychological medicine, vol. 44, pp. 2833-43, oct 2014.

[162] L. Wang, C.-Y. Wee, H.-I. Suk, X. Tang, and D. Shen, "MRI-based intelligence quotient (IQ) estimation with sparse learning.," PloS one, vol. 10, no. 3, p. e0117295, 2015.

[163] M. Vitali, C. I. Ripamonti, F. Roila, C. Proto, D. Signorelli, M. Imbimbo, G. Corrao, A. Brissa, G. Rosaria, F. de Braud, M. C. Garassino, and G. Lo Russo, "Cognitive impairment and chemotherapy: a brief overview," Critical Reviews in Oncology/Hematology, vol. 118, pp. 7-14, oct 2017.

[164] R. C. K. Chan, M. K. Lai, and I. H. Robertson, "Latent structure of the Test of Everyday Attention in a non-clinical Chinese sample," Archives of Clinical Neuropsychology, vol. 21, pp. 477-485, aug 2006.

[165] J. Vardy, S. Rourke, and I. F. Tannock, "Evaluation of cognitive function associated with chemotherapy: A review of published studies and recommendations for future research," Journal of Clinical Oncology, vol. 25, pp. 2455-2463, jun 2007.

[166] G. Winocur, H. Berman, M. Nguyen, M. A. Binns, M. Henkelman, M. van Eede, M. PiquetteMiller, M. J. Sekeres, J. M. Wojtowicz, J. Yu, H. Zhang, and I. F. Tannock, "Neurobiological Mechanisms of Chemotherapy-induced Cognitive Impairment in a Transgenic Model of Breast Cancer," Neuroscience, vol. 369, pp. 51-65, jan 2018.

[167] S. Menning, M. B. de Ruiter, D. J. Veltman, W. Boogerd, H. S. Oldenburg, L. Reneman, and S. B. Schagen, "Changes in brain white matter integrity after systemic treatment for breast cancer: a prospective longitudinal study," Brain Imaging and Behavior, pp. 1-11, 2017.

[168] E. Grebenciucova and J. R. Berger, "Immunosenescence: the Role of Aging in the Predisposition to Neuro-Infectious Complications Arising from the Treatment of Multiple Sclerosis.," Current neurology and neuroscience reports, vol. 17, p. 61, aug 2017.

[169] D. Raj, Z. Yin, M. Breur, J. Doorduin, I. R. Holtman, M. Olah, I. J. Mantingh-Otter, D. Van Dam, P. P. De Deyn, W. den Dunnen, B. J. L. Eggen, S. Amor, and E. Boddeke, "Increased White Matter Inflammation in Aging- and Alzheimer's Disease Brain," Frontiers in Molecular Neuroscience, vol. 10, no. June, pp. 1-18, 2017.

[170] N. Salvadores, M. Sanhueza, P. Manque, and F. A. Court, "Axonal degeneration during aging and its functional role in neurodegenerative disorders," Frontiers in Neuroscience, vol. 11, no. SEP, 2017. 
[171] M. R. Damani, L. Zhao, A. M. Fontainhas, J. Amaral, R. N. Fariss, and W. T. Wong, "Age-related alterations in the dynamic behavior of microglia.," Aging cell, vol. 10, pp. 263-76, apr 2011.

[172] C. Zhao, W.-W. Li, and R. J. Franklin, "Differences in the early inflammatory responses to toxin-induced demyelination are associated with the age-related decline in CNS remyelination," Neurobiology of Aging, vol. 27, pp. 1298-1307, sep 2006.

[173] I. Sack, B. Beierbach, J. Wuerfel, D. Klatt, U. Hamhaber, S. Papazoglou, P. Martus, and J. Braun, "The impact of aging and gender on brain viscoelasticity," Neurolmage, vol. 46, no. 3, pp. 652-657, 2009.

[174] M. J. Simon and J. J. Iliff, "Regulation of cerebrospinal fluid (CSF) flow in neurodegenerative, neurovascular and neuroinflammatory disease," Biochimica et Biophysica Acta (BBA) - Molecular Basis of Disease, vol. 1862, pp. 442-451, mar 2016.

[175] M.-A. Arevalo, I. Azcoitia, and L. M. Garcia-Segura, "The neuroprotective actions of oestradiol and oestrogen receptors.," Nature reviews. Neuroscience, vol. 16, pp. 17-29, jan 2015.

[176] A. Turken, S. Whitfield-Gabrieli, R. Bammer, J. V. Baldo, N. F. Dronkers, and J. D. E. Gabrieli, "Cognitive processing speed and the structure of white matter pathways: convergent evidence from normal variation and lesion studies.," Neurolmage, vol. 42, pp. 1032-44, aug 2008.

[177] W. V. Hecke, G. Nagels, A. Leemans, E. Vandervliet, J. Sijbers, and P. M. Parizel, "Correlation of cognitive dysfunction and diffusion tensor MRI measures in patients with mild and moderate multiple sclerosis," Journal of Magnetic Resonance Imaging, vol. 31, pp. 1492-1498, may 2010.

[178] M. F. Kraus, T. Susmaras, B. P. Caughlin, C. J. Walker, J. A. Sweeney, and D. M. Little, "White matter integrity and cognition in chronic traumatic brain injury: a diffusion tensor imaging study," Brain, vol. 130, pp. 2508-2519, oct 2007.

[179] F. W. Boele, C. M. T. Schilder, M.-L. de Roode, J. B. Deijen, and S. B. Schagen, "Cognitive functioning during long-term tamoxifen treatment in postmenopausal women with breast cancer.," Menopause (New York, N.Y.), vol. 22, pp. 17-25, jan 2015.

[180] N. Seliktar, C. Polek, A. Brooks, and T. Hardie, "Cognition in breast cancer survivors: hormones versus depression.," Psycho-oncology, vol. 24, pp. 402-7, apr 2015.

[181] C. Andreotti, J. C. Root, T. A. Ahles, B. S. McEwen, and B. E. Compas, "Cancer, coping, and cognition: a model for the role of stress reactivity in cancer-related cognitive decline," Psycho-Oncology, vol. 24, pp. 617-623, jun 2015.

[182] S. Menning, M. B. de Ruiter, D. J. Veltman, V. Koppelmans, C. Kirschbaum, W. Boogerd, L. Reneman, and S. B. Schagen, "Multimodal MRI and cognitive function in patients with breast cancer prior to adjuvant treatment - The role of fatigue," Neurolmage: Clinical, vol. 7 , pp. 547-554, 2015. 
[183] K. Van Calsteren, R. Verbesselt, N. Ottevanger, M. Halaska, L. Heyns, R. Van Bree, E. de Bruijn, D. Chai, M. Delforge, L. Noens, V. Renard, E. Witteveen, L. Rob, J. de Hoon, and F. Amant, "Pharmacokinetics of chemotherapeutic agents in pregnancy: a preclinical and clinical study.," Acta obstetricia et gynecologica Scandinavica, vol. 89, no. 10, pp. 1338-1345, 2010.

[184] J. Richetto and M. A. Riva, "Prenatal maternal factors in the development of cognitive impairments in the offspring," Journal of Reproductive Immunology, vol. 104-105, no. C, pp. 20-25, 2014.

[185] M. Mennes, P. Stiers, L. Lagae, and B. Van den Bergh, "Long-term cognitive sequelae of antenatal maternal anxiety: involvement of the orbitofrontal cortex.," Neuroscience and biobehavioral reviews, vol. 30, no. 8, pp. 1078-86, 2006.

[186] M. Mennes, B. V. den Bergh, L. Lagae, and P. Stiers, "Developmental brain alterations in 17 year old boys are related to antenatal maternal anxiety," Clinical Neurophysiology, vol. 120, pp. 1116-1122, jun 2009.

[187] D. Wechsler, "Wechsler intelligence scale for children-Third edition," San Antonio, TX: The Psychological Corporation, 1991.

[188] D. Wechsler, Wechsler intelligence scale for children-fifth edition. Bloomington, MN: Pearson, 2014.

[189] I. Winkler, S. Haufe, and M. Tangermann, "Automatic Classification of Artifactual ICAComponents for Artifact Removal in EEG Signals," Behavioral and Brain Functions, vol. 7, no. 1, p. 30, 2011.

[190] I. Winkler, S. Brandl, F. Horn, E. Waldburger, C. Allefeld, and M. Tangermann, "Robust artifactual independent component classification for $\mathrm{BCl}$ practitioners," Journal of Neural Engineering, vol. 11, no. 3, p. 35013, 2014.

[191] W. De Clercq, A. Vergult, B. Vanrumste, W. Van Paesschen, and S. Van Huffel, "Canonical Correlation Analysis Applied to Remove Muscle Artifacts From the Electroencephalogram," IEEE Transactions on Biomedical Engineering, vol. 53, pp. 2583-2587, nov 2006.

[192] J. A. Urigüen and B. Garcia-Zapirain, "EEG artifact removal - State-of-the-art and guidelines," Journal of Neural Engineering, 2015.

[193] L. T. Trujillo, S. Kornguth, and D. M. Schnyer, "An ERP examination of the different effects of sleep deprivation on exogenously cued and endogenously cued attention.," Sleep, vol. 32, no. 10, pp. 1285-97, 2009.

[194] M. Schrooten, E. G. Ghumare, L. Seynaeve, T. Theys, P. Dupont, W. Van Paesschen, and R. Vandenberghe, "Electrocorticography of Spatial Shifting and Attentional Selection in Human Superior Parietal Cortex," Frontiers in Human Neuroscience, vol. 11, pp. 1-12, may 2017. 
[195] M. Arnold, H. E. Karim-Kos, J. W. Coebergh, G. Byrnes, A. Antilla, J. Ferlay, A. G. Renehan, D. Forman, and I. Soerjomataram, "Recent trends in incidence of five common cancers in 26 European countries since 1988: Analysis of the European Cancer Observatory," European Journal of Cancer, vol. 51, pp. 1164-1187, jun 2015.

[196] J. Blommaert, R. Zink, S. Deprez, I. Myatchin, P. Dupont, T. Vandenbroucke, C. Sleurs, K. Van Calsteren, F. Amant, and L. Lagae, "Long-term impact of prenatal exposure to chemotherapy on executive functioning: An ERP study," Clinical Neurophysiology, 2019.

[197] C. Maggen, V. E. Wolters, E. Cardonick, M. Fumagalli, M. J. Halaska, C. A. Lok, J. de Haan, K. Van Tornout, K. Van Calsteren, and F. Amant, "Pregnancy and Cancer: the INCIP Project," Current Oncology Reports, vol. 22, no. 2, 2020.

[198] M. van Gerwen, T. Vandenbroucke, M. Verheecke, K. Van Calsteren, M. J. Halaska, M. Fumagalli, R. Fruscio, A. Gandhi, M. Veening, L. Lagae, P. B. Ottevanger, J.-U. Voigt, J. de Haan, M. M. Gziri, C. Maggen, L. Mertens, G. Naulaers, L. Claes, and F. Amant, "Data describing child development at 6 years after maternal cancer diagnosis and treatment during pregnancy," Data in Brief, vol. 32, p. 106209, oct 2020.

[199] T. M. Achenbach, "Child Behavior Checklist," in Encyclopedia of Clinical Neuropsychology, pp. 546-552, New York, NY: Springer New York, 2011.

[200] G. A. Gioia, P. K. Isquith, and R. M. Roth, "Behavior Rating Inventory for Executive Function," in Encyclopedia of Clinical Neuropsychology, pp. 532-538, Cham: Springer International Publishing, 2018.

[201] J. Gardosi, A. Francis, S. Turner, and M. Williams, "Customized growth charts: rationale, validation and clinical benefits," American Journal of Obstetrics and Gynecology, vol. 218, pp. S609-S618, feb 2018.

[202] J. Veraart, D. S. Novikov, D. Christiaens, B. Ades-aron, J. Sijbers, and E. Fieremans, "Denoising of diffusion MRI using random matrix theory," Neurolmage, vol. 142, pp. 394-406, nov 2016.

[203] E. Kellner, B. Dhital, V. G. Kiselev, and M. Reisert, "Gibbs-ringing artifact removal based on local subvoxel-shifts," Magnetic Resonance in Medicine, vol. 76, pp. 1574-1581, nov 2016.

[204] J. L. Andersson, S. Skare, and J. Ashburner, "How to correct susceptibility distortions in spin-echo echo-planar images: application to diffusion tensor imaging," Neurolmage, vol. 20, pp. 870-888, oct 2003.

[205] J.-D. Tournier, R. E. Smith, D. A. Raffelt, R. Tabbara, T. Dhollander, M. Pietsch, D. Christiaens, B. Jeurissen, C.-H. Yeh, and A. Connelly, "MRtrix3: A fast, flexible and open software framework for medical image processing and visualisation," bioRxiv, 2019. 
[206] S. Whitfield-Gabrieli and A. Nieto-Castanon, "Conn : A Functional Connectivity Toolbox for Correlated and Anticorrelated Brain Networks," Brain Connectivity, vol. 2, pp. 125-141, jun 2012.

[207] Y. Wang, E. Ghumare, R. Vandenberghe, and P. Dupont, "Comparison of Different Generalizations of Clustering Coefficient and Local Efficiency for Weighted Undirected Graphs," Neural Computation, vol. 29, pp. 313-331, feb 2017.

[208] R. E. Smith, T. Dhollander, and A. Connelly, "On the regression of intracranial volume in Fixel-Based Analysis Discussion / Conclusion," in Proceedings of the International Society for Magnetic Resonance in Medicine, vol. 27, p. 3385, 2019.

[209] D. A. Raffelt, R. E. Smith, G. R. Ridgway, J. D. Tournier, D. N. Vaughan, S. Rose, R. Henderson, and A. Connelly, "Connectivity-based fixel enhancement: Whole-brain statistical analysis of diffusion MRI measures in the presence of crossing fibres," Neurolmage, vol. 117, pp. 40-55, aug 2015.

[210] R. Wright, V. Kyriakopoulou, C. Ledig, M. Rutherford, J. Hajnal, D. Rueckert, and P. Aljabar, "Automatic quantification of normal cortical folding patterns from fetal brain MRI," Neurolmage, vol. 91, pp. 21-32, may 2014.

[211] P. A. Habas, J. A. Scott, A. Roosta, V. Rajagopalan, K. Kim, F. Rousseau, A. J. Barkovich, O. A. Glenn, and C. Studholme, "Early Folding Patterns and Asymmetries of the Normal Human Brain Detected from in Utero MRI," Cerebral Cortex, vol. 22, pp. 13-25, jan 2012.

[212] A. D. Friederici, N. Chomsky, R. C. Berwick, A. Moro, and J. J. Bolhuis, "Language, mind and brain," Nature Human Behaviour, vol. 1, no. 10, pp. 713-722, 2017.

[213] C. Köhler, P. Oppelt, G. Favero, B. Morgenstern, I. Runnebaum, A. Tsunoda, A. Schmittel, A. Schneider, M. Mueller, and S. Marnitz, "How much platinum passes the placental barrier? Analysis of platinum applications in 21 patients with cervical cancer during pregnancy," American Journal of Obstetrics and Gynecology, vol. 213, pp. 206.e1-206.e5, aug 2015.

[214] A. Yancey, M. S. Harris, A. Egbelakin, J. Gilbert, D. B. Pisoni, and J. Renbarger, "Risk factors for cisplatin-associated ototoxicity in pediatric oncology patients," Pediatric Blood \& Cancer, vol. 59, pp. 144-148, jul 2012.

[215] S. Hao, X. Jiang, A. Yan, and B. Yang, "Perinatal cisplatin exposure induces cochlear apoptosis in newborn guinea pigs," Archives of Toxicology, vol. 85, pp. 19-25, jan 2011.

[216] E. C. Geijteman, C. W. Wensveen, J. J. Duvekot, and L. van Zuylen, "A Child With Severe Hearing Loss Associated With Maternal Cisplatin Treatment During Pregnancy," Obstetrics \& Gynecology, vol. 124, pp. 454-456, aug 2014.

[217] N. Garg, S. W. Reddel, D. H. Miller, J. Chataway, D. S. Riminton, Y. Barnett, L. Masters, M. H. Barnett, and T. A. Hardy, "The corpus callosum in the diagnosis of multiple sclerosis and 
other CNS demyelinating and inflammatory diseases," Journal of Neurology, Neurosurgery and Psychiatry, vol. 86, no. 12, pp. 1374-1382, 2015.

[218] C. Clouchoux, D. Kudelski, A. Gholipour, S. K. Warfield, S. Viseur, M. Bouyssi-Kobar, J.-L. Mari, A. C. Evans, A. J. du Plessis, and C. Limperopoulos, "Quantitative in vivo MRI measurement of cortical development in the fetus," Brain Structure and Function, vol. 217, pp. 127-139, jan 2012.

[219] F. Amant, P. Berveiller, I. Boere, E. Cardonick, R. Fruscio, M. Fumagalli, M. J. Halaska, A. Hasenburg, A. L. V. Johansson, M. Lambertini, C. Lok, C. Maggen, P. Morice, F. Peccatori, P. Poortmans, K. Van Calsteren, T. Vandenbroucke, M. van Gerwen, M. van den Heuvel-Eibrink, F. Zagouri, and I. Zapardiel, "Gynecologic cancers in pregnancy: guidelines based on a third international consensus meeting," Annals of Oncology, 2019.

[220] M. Lishner, I. Avivi, J. F. Apperley, D. Dierickx, A. M. Evens, M. Fumagalli, I. Nulman, F. S. Oduncu, F. A. Peccatori, S. Robinson, K. Van Calsteren, T. Vandenbroucke, F. Van den Heuvel, and F. Amant, "Hematologic Malignancies in Pregnancy: Management Guidelines From an International Consensus Meeting," Journal of Clinical Oncology, vol. 34, pp. 501-508, feb 2016.

[221] F. Amant, S. Deckers, K. Van Calsteren, S. Loibl, M. Halaska, L. Brepoels, J. Beijnen, F. Cardoso, O. Gentilini, L. Lagae, O. Mir, P. Neven, N. Ottevanger, S. Pans, F. Peccatori, R. Rouzier, H.-J. Senn, H. Struikmans, M.-R. Christiaens, D. Cameron, and A. Du Bois, "Breast cancer in pregnancy: Recommendations of an international consensus meeting," European Journal of Cancer, vol. 46, pp. 3158-3168, dec 2010.

[222] K. Van der Gucht, S. Ahmadoun, M. Melis, E. de Cloe, C. Sleurs, A. Radwan, J. Blommaert, K. Takano, M. Vandenbulcke, H. Wildiers, P. Neven, P. Kuppens, F. Raes, A. Smeets, S. Sunaert, and S. Deprez, "Effects of a mindfulness-based intervention on cancer-related cognitive impairment: Results of a randomized controlled functional magnetic resonance imaging pilot study," Cancer.

[223] J. P. Vogel, S. Chawanpaiboon, A.-B. Moller, K. Watananirun, M. Bonet, and P. Lumbiganon, "The global epidemiology of preterm birth," Best Practice \& Research Clinical Obstetrics \& Gynaecology, vol. 52, pp. 3-12, oct 2018.

[224] T. Vandenbroucke, S. N. Han, K. Van Calsteren, T. F. Wilderjans, B. R. Van den Bergh, L. Claes, and F. Amant, "Psychological distress and cognitive coping in pregnant women diagnosed with cancer and their partners," Psycho-Oncology, vol. 26, no. 8, pp. 1215-1221, 2017.

[225] S. Aoyagi and K. J. Tsuchiya, "Does maternal postpartum depression affect children's developmental outcomes?," Journal of Obstetrics and Gynaecology Research, vol. 45, pp. 1809-1820, sep 2019.

[226] I. Landi, M. Giannotti, P. Venuti, and S. Falco, "Maternal and family predictors of infant psychological development in at-risk families: A multilevel longitudinal study," Research in Nursing \& Health, vol. 43, pp. 17-27, feb 2020. 
[227] D. Van Essen, K. Ugurbil, E. Auerbach, D. Barch, T. Behrens, R. Bucholz, A. Chang, L. Chen, M. Corbetta, S. Curtiss, S. Della Penna, D. Feinberg, M. Glasser, N. Harel, A. Heath, L. Larson-Prior, D. Marcus, G. Michalareas, S. Moeller, R. Oostenveld, S. Petersen, F. Prior, B. Schlaggar, S. Smith, A. Snyder, J. Xu, and E. Yacoub, "The Human Connectome Project: A data acquisition perspective," Neurolmage, vol. 62, pp. 2222-2231, oct 2012.

[228] B. Casey, T. Cannonier, M. I. Conley, A. O. Cohen, D. M. Barch, M. M. Heitzeg, M. E. Soules, T. Teslovich, D. V. Dellarco, H. Garavan, C. A. Orr, T. D. Wager, M. T. Banich, N. K. Speer, M. T. Sutherland, M. C. Riedel, A. S. Dick, J. M. Bjork, K. M. Thomas, B. Chaarani, M. H. Mejia, D. J. Hagler, M. Daniela Cornejo, C. S. Sicat, M. P. Harms, N. U. Dosenbach, M. Rosenberg, E. Earl, H. Bartsch, R. Watts, J. R. Polimeni, J. M. Kuperman, D. A. Fair, and A. M. Dale, "The Adolescent Brain Cognitive Development (ABCD) study: Imaging acquisition across 21 sites," Developmental Cognitive Neuroscience, vol. 32, pp. 43-54, aug 2018.

[229] C. Geng, M. Moteabbed, J. Seco, Y. Gao, X. George Xu, J. Ramos-Méndez, B. Faddegon, and $\mathrm{H}$. Paganetti, "Dose assessment for the fetus considering scattered and secondary radiation from photon and proton therapy when treating a brain tumor of the mother," Physics in Medicine and Biology, vol. 61, no. 2, pp. 683-695, 2015.

[230] X. Wang, F. Poenisch, N. Sahoo, R. X. Zhu, M. Lii, M. T. Gillin, J. Li, and D. Grosshans, "Spot scanning proton therapy minimizes neutron dose in the setting of radiation therapy administered during pregnancy," Journal of Applied Clinical Medical Physics, vol. 17, pp. 366-376, sep 2016.

[231] J. F. Fagan, C. R. Holland, and K. Wheeler, "The prediction, from infancy, of adult IQ and achievement," Intelligence, vol. 35, pp. 225-231, may 2007.

[232] D. Christiaens, P. J. Slator, L. Cordero-Grande, A. N. Price, M. Deprez, D. C. Alexander, M. Rutherford, J. V. Hajnal, and J. Hutter, "In utero diffusion MRI: challenges, advances, and applications," no. June, 2019.

[233] M. Bastiani, M. Cottaar, S. P. Fitzgibbon, S. Suri, F. Alfaro-Almagro, S. N. Sotiropoulos, S. Jbabdi, and J. L. Andersson, "Automated quality control for within and between studies diffusion MRI data using a non-parametric framework for movement and distortion correction," Neurolmage, vol. 184, pp. 801-812, jan 2019.

[234] M. Eyre, S. P. Fitzgibbon, J. Ciarrusta, L. Cordero-Grande, A. N. Price, T. Poppe, A. Schuh, E. Hughes, C. O'Keeffe, J. Brandon, D. Cromb, K. Vecchiato, J. Andersson, E. P. Duff, S. J. Counsell, S. M. Smith, D. Rueckert, J. V. Hajnal, T. Arichi, J. O'Muircheartaigh, D. Batalle, and A. D. Edwards, "The developing human connectome project: typical and disrupted perinatal functional connectivity," bioRxiv, 2020.

[235] D. Fenchel, R. Dimitrova, J. Seidlitz, E. C. Robinson, D. Batalle, J. Hutter, D. Christiaens, M. Pietsch, J. Brandon, E. J. Hughes, J. Allsop, C. O'Keeffe, A. N. Price, L. Cordero-Grande, 
A. Schuh, A. Makropoulos, J. Passerat-Palmbach, J. Bozek, D. Rueckert, J. V. Hajnal, A. Raznahan, G. McAlonan, A. D. Edwards, and J. O'Muircheartaigh, "Development of microstructural and morphological cortical profiles in the neonatal brain," bioRxiv, 2020.

[236] C. Laule and G. W. Moore, "Myelin water imaging to detect demyelination and remyelination and its validation in pathology," Brain Pathology, vol. 28, pp. 750-764, sep 2018.

[237] H. Zhu and P. B. Barker, "MR Spectroscopy and Spectroscopic Imaging of the Brain," pp. 203-226, 2011.

[238] M. Mikkelsen, P. B. Barker, P. K. Bhattacharyya, M. K. Brix, P. F. Buur, K. M. Cecil, K. L. Chan, D. Y.-T. Chen, A. R. Craven, K. Cuypers, M. Dacko, N. W. Duncan, U. Dydak, D. A. Edmondson, G. Ende, L. Ersland, F. Gao, I. Greenhouse, A. D. Harris, N. He, S. Heba, N. Hoggard, T.-W. Hsu, J. F. Jansen, A. Kangarlu, T. Lange, R. M. Lebel, Y. Li, C.-Y. E. Lin, J.-K. Liou, J.-F. Lirng, F. Liu, R. Ma, C. Maes, M. Moreno-Ortega, S. O. Murray, S. Noah, R. Noeske, M. D. Noseworthy, G. Oeltzschner, J. J. Prisciandaro, N. A. Puts, T. P. Roberts, M. Sack, N. Sailasuta, M. G. Saleh, M.-P. Schallmo, N. Simard, S. P. Swinnen, M. Tegenthoff, P. Truong, G. Wang, I. D. Wilkinson, H.-J. Wittsack, H. Xu, F. Yan, C. Zhang, V. Zipunnikov, H. J. Zöllner, and R. A. Edden, "Big GABA: Edited MR spectroscopy at 24 research sites," Neurolmage, vol. 159, pp. 32-45, oct 2017.

[239] J. Z. Kiss, L. Vasung, and V. Petrenko, "Process of cortical network formation and impact of early brain damage," Current Opinion in Neurology, vol. 27, no. 2, pp. 133-141, 2014.

[240] K. J. Gorgolewski, T. Auer, V. D. Calhoun, R. C. Craddock, S. Das, E. P. Duff, G. Flandin, S. S. Ghosh, T. Glatard, Y. O. Halchenko, D. A. Handwerker, M. Hanke, D. Keator, X. Li, Z. Michael, C. Maumet, B. N. Nichols, T. E. Nichols, J. Pellman, J.-B. Poline, A. Rokem, G. Schaefer, V. Sochat, W. Triplett, J. A. Turner, G. Varoquaux, and R. A. Poldrack, "The brain imaging data structure, a format for organizing and describing outputs of neuroimaging experiments," Scientific Data, vol. 3, p. 160044, dec 2016.

[241] V. A. Magnotta and L. Friedman, "Measurement of Signal-to-Noise and Contrast-to-Noise in the fBIRN Multicenter Imaging Study," Journal of Digital Imaging, vol. 19, pp. 140-147, jun 2006.

[242] M. Ganzetti, N. Wenderoth, and D. Mantini, "Intensity Inhomogeneity Correction of Structural MR Images: A Data-Driven Approach to Define Input Algorithm Parameters," Frontiers in Neuroinformatics, vol. 10, p. 10, mar 2016.

[243] D. Atkinson, D. Hill, P. Stoyle, P. Summers, and S. Keevil, "Automatic correction of motion artifacts in magnetic resonance images using an entropy focus criterion," IEEE Transactions on Medical Imaging, vol. 16, pp. 903-910, dec 1997. 
[244] J. D. Power, K. A. Barnes, A. Z. Snyder, B. L. Schlaggar, and S. E. Petersen, "Spurious but systematic correlations in functional connectivity MRI networks arise from subject motion," Neurolmage, vol. 59, pp. 2142-2154, feb 2012.

[245] G. Krüger and G. H. Glover, "Physiological noise in oxygenation-sensitive magnetic resonance imaging," Magnetic Resonance in Medicine, vol. 46, pp. 631-637, oct 2001.

[246] N. J. Tustison, P. A. Cook, A. Klein, G. Song, S. R. Das, J. T. Duda, B. M. Kandel, N. van Strien, J. R. Stone, J. C. Gee, and B. B. Avants, "Large-scale evaluation of ANTs and FreeSurfer cortical thickness measurements," Neurolmage, vol. 99, pp. 166-179, oct 2014.

[247] F. Isensee, M. Schell, I. Pflueger, G. Brugnara, D. Bonekamp, U. Neuberger, A. Wick, H. Schlemmer, S. Heiland, W. Wick, M. Bendszus, K. H. Maier-Hein, and P. Kickingereder, "Automated brain extraction of multisequence MRI using artificial neural networks.," Human brain mapping, vol. 40, pp. 4952-4964, dec 2019.

[248] L. L. Backhausen, M. M. Herting, J. Buse, V. Roessner, M. N. Smolka, and N. C. Vetter, "Quality Control of Structural MRI Images Applied Using FreeSurfer-A Hands-On Workflow to Rate Motion Artifacts," Frontiers in Neuroscience, vol. 10, p. 558, dec 2016.

[249] R. S. Desikan, F. Ségonne, B. Fischl, B. T. Quinn, B. C. Dickerson, D. Blacker, R. L. Buckner, A. M. Dale, R. P. Maguire, B. T. Hyman, M. S. Albert, and R. J. Killiany, "An automated labeling system for subdividing the human cerebral cortex on MRI scans into gyral based regions of interest.," Neurolmage, vol. 31, pp. 968-80, jul 2006.

[250] B. Fischl, D. H. Salat, A. J. van der Kouwe, N. Makris, F. Ségonne, B. T. Quinn, and A. M. Dale, "Sequence-independent segmentation of magnetic resonance images," Neurolmage, vol. 23, pp. S69-S84, jan 2004.

[251] R. E. Smith, J.-D. Tournier, F. Calamante, and A. Connelly, "SIFT: Spherical-deconvolution informed filtering of tractograms," Neurolmage, vol. 67, pp. 298-312, feb 2013.

[252] K. Pannek, J. Fripp, J. M. George, S. Fiori, P. B. Colditz, R. N. Boyd, and S. E. Rose, "Fixel-based analysis reveals alterations is brain microstructure and macrostructure of preterm-born infants at term equivalent age," Neurolmage: Clinical, vol. 18, no. September 2017, pp. 51-59, 2018.

[253] S. Wang, D. J. Peterson, J. C. Gatenby, W. Li, T. J. Grabowski, and T. M. Madhyastha, "Evaluation of Field Map and Nonlinear Registration Methods for Correction of Susceptibility Artifacts in Diffusion MRI," Frontiers in Neuroinformatics, vol. 11, p. 17, feb 2017. 


\section{CURRICULUM VITAE}

Email: jeroen.blommaert@kuleuven.be

blommaertjeroen@gmail.com

Date of birth: 29 April 1993

Nationality: Belgian

Work phone: 016340408

Mobile phone: +32 (0) 499341459

Adress: $\quad$ Sneppenstraat 23, 3010 Kessel-lo, Belgium

Drivers Licence B

be.linkedin.com/in/jeroenblommaert

www.researchgate.net/profile/Jeroen_Blommaert2 


\section{Career \& education}

2020-present Master of science in medical radiation physics, KU Leuven

2016-present PhD researcher at KU Leuven.

- "Effects of prenatal exposure to cancer treatment on neurocognitive development: an MRI and ERP study"

- Promotors: Prof. Frédéric amant, Dr. Sabine Deprez and Prof. Kristel Van Calsteren

- Doctoral school of biomedical sciences, Cognitive and Molecular Neurosciences

- 2018-present: Aspirant researcher for the Research Foundation Flanders (FWO, 22\% of applicants receive this grant)

2014-2016 Master of science in biomedical engineering, KU Leuven. Graduated cum laude $(74 \%)$

- Master thesis: Investigation of microaggregate fusion kinetics during chondrogenic differentiation of hPDCs

- Internship at MTEC, Thailand (6 weeks): Customized design and fabrication of wrist splints using additive manufacturing

2011-2014 Bachelor of science in mechanical and electrical engineering, KU Leuven. Graduated cum laude (72\%) 


\title{
Workshops\& courses
}

2020 ECMRN pre-congress (fetal, neonatal and infant MRI), Marseille, France

2020 Interactive Training in Applied GCP for Investigators \& Site Personnel, UZ Leuven, Leuven

2019 MRtrix3 workshop, Antwerp, Belgium.

2018;2019 OHBM educational courses, Singapore; Rome, Italy.

$2019 \quad$ ISMRM educational courses, Montréal, Canada.

2018 Writing for medical journals, Stuart Spencer, Leuven, Belgium.

2017 Exploring the Human Connectome: fundamentals of network science and graph theory for brain networks, Utrecht university, Netherlands.

2017 Edinburgh SPM course 2017, University of Edinburgh, UK.

$2016 \quad$ Univariate data and modelling, KU Leuven, Leuven, Belgium.

2016 Advanced medical imaging, KU Leuven, Leuven, Belgium.

2016 Biomedical databases and databases evidence-based medicine/practice, university library, Leuven, Belgium.

\section{Reviewer of Journal Articles}

\author{
2020 Mindfulness \\ 2020 Scientific Reports
}

\section{Guidance of Master theses}

2019-2021 Master of science in psychology

Emma Masselis: Cortical development in childhood cancer patients

\section{Jury member of Master theses}

2020 Master of science in speech language pathology and audiology 


\section{Guidance of Bachelor students}

2017 Bachelor of science in biomedical sciences

Shani Decoster: internship as student-researcher

\section{Awards and grants}

2020

2020 FWO travel grant for participation in the 7th Biennial International Cancer \& Cognition Taskforce Meeting, Denver, CO, USA

2018-2022 FWO aspirant fellowship (11B9919N): 'Cancer and pregnancy: effects of prenatal exposure to cancer treatment on neurocognitive development: an MRI and ERP study'

2015 University Centre for Development Cooperation (UCOS) travel grant for a 6-week internship at the National Research Center for Material and Metal Technology (MTEC), Pathun Thani, Thailand

\section{Journal articles}

1. Blommaert J., Radwan A., Sleurs C., Maggen C., van Gerwen M., Wolters V. Christiaens D., Peeters R., Dupont P., Sunaert S., Van Calsteren K., Deprez S. and Amant F. (accepted). The impact of cancer and chemotherapy during pregnancy on child neurodevelopment: a multimodal neuroimaging analysis, EClinicalMedicine

2. Radwan A., Emsell L, Blommaert J., Zhylka A., Kovacs S., Theys T., Sollmann N., Dupont P., Sunaert S. (2020). Virtual brain grafting: Enabling whole brain parcellation in the presence of large lesions, medRxiv. doi: 10.1101/2020.09.30.20204701

3. Schroyen G., Meylaers M., Deprez S., Blommaert J., Smeets A., Jacobs S., Sunaert S., Sleurs C., Uyttebroeck A. (2020). Prevalence of leukoencephalopathy and its potential cognitive sequelae in cancer patients, Journal Of Chemotherapy. doi: 10.1080/1120009X.2020.1805239

4. Van der Gucht, K., Ahmadoun, S., Melis, M., de Cloe, E., Sleurs, C., Radwan, A., Blommaert, J., Takano, K., Vandenbulcke, M., Wildiers, H., Neven, P., Kuppens, P., 
Raes, F., Smeets, A., Sunaert, S., Deprez, S. (2020). Effects of a mindfulness-based intervention on cancer-related cognitive impairment: Results of a randomized controlled functional magnetic resonance imaging pilot study. Cancer, 1-10. doi: 10.1002/cncr.33074

5. De Vocht, J., Blommaert, J., Devrome, M., Radwan, A., Van Weehaeghe, D., De Schaepdryver, M., Ceccarini, J., Rezaei, A., Schramm, G., van Aalst, J., Chiò, A., Pagani, M., Stam, D., Van Esch, H., Lamaire, N., Verhaegen, M., Mertens, N., Poesen, K., van den Berg, L.H., van Es, M.A., Vandenberghe, R., Vandenbulcke, M., Van den Stock, J., Koole, M., Dupont, P., Van Laere, K., Van Damme, P. (2020). Use of Multimodal Imaging and Clinical Biomarkers in Presymptomatic Carriers of C9orf72 Repeat Expansion. JAMA Neurol. doi: 10.1001/jamaneurol.2020.1087

6. Sleurs, C., Blommaert, J., Batalle, D., Verly, M., Sunaert, S., Peeters, R., Lemiere, J., Uyttebroeck, A., Deprez, S. (2020). Cortical thinning and altered functional brain coherence in survivors of childhood sarcoma. Brain Imaging And Behavior. doi: $10.1007 /$ s11682-020-00276-9

7. Mailleux, L., Simon Martinez, C., Radwan, A., Blommaert, J., Gooijers, J., Wenderoth, N., Klingels, K., Ortibus, E., Sunaert, S., Feys, H. (2020). White matter characteristics of motor, sensory and interhemispheric tracts underlying impaired upper limb function in children with unilateral cerebral palsy. Brain Structure \& Function. doi: 10.1007/s00429020-02070-1

8. Blommaert, J., Schroyen, G., Vandenbulcke, M., Radwan, A., Smeets, A., Peeters, R., Sleurs, C., Neven, P., Wildiers, H., Amant, F., Sunaert, S., Deprez, S. (2020). Veranderingen in hersenvolume en cognitief functioneren na chemotherapie voor borstkanker zijn leeftijdsafhankelijk. Oncohemato, 13 (7), 21-24.

9. Blommaert, J., Zink, R., Deprez, S., Myatchin, I., Dupont, P., Vandenbroucke, T., Sleurs, C., Van Calsteren, K., Amant, F., Lagae, L. (2019). Long-term impact of prenatal exposure to chemotherapy on executive functioning: An ERP study. ClinicaL Neurophysiology, 130 (9), 1655-1664. doi: 10.1016/j.clinph.2019.06.012

10. Blommaert, J., Schroyen, G., Vandenbulcke, M., Radwan, A., Smeets, A., Peeters, R., Sleurs, C., Neven, P., Wildiers, H., Amant, F., Sunaert, S., Deprez, S. (2019). Age-dependent brain volume and neuropsychological changes after chemotherapy in breast cancer patients. Human Brain Mapping, 40 (17), 4994-5010. doi: 10.1002/hbm.24753

11. Simon-Martinez, C., Jaspers, E., Alaerts, K., Ortibus, E., Balsters, J., Mailleux, L., Blommaert, J., Sleurs, C., Klingels, K., Amant, F., Uyttebroeck, A., Wenderoth, N., Feys, H. (2019). Influence of the corticospinal tract wiring pattern on sensorimotor 
functional connectivity and clinical correlates of upper limb function in unilateral cerebral palsy. Scientific Reports, 9, Art.No. ARTN 8230. doi: 10.1038/s41598-019-44728-9

\section{Abstracts}

1. Sleurs, C., Lemiere, J., Blommaert, J., Deprez, S., Van Beek, K., Uyttebroeck, A., Jacobs, S. (2020). Cortical volume and thickness in adult survivors of childhood posterior fossa tumors. Presented at the International Symposium on Pediatric Neuro-Oncology, Karuizawa, Japan, 13 Dec 2020-16 Dec 2020.

2. Schroyen G., Blommaert J., Radwan A., Smeets A., Van Laere K., Deprez S., Sunaert S. (2020). Morphological changes after chemotherapy treatment for breast cancer. Presented at the Organization for Human Brain Mapping, online, 23 Jun 2020-03 Jul 2020.

3. Melis, M., Van der Gucht, K., Ahmadoun, S., de Cloe, E., Smeets, A., Vandenbulcke, M., Wildiers, H., Neven, P., Keisuke, T., Sleurs, C., Blommaert, J., Radwan, A., Kuppens, P., Raes, F., Sunaert, S., Deprez, S. (2020). An MRI pilot study on mindfulness and cognitive impairment after breast cancer treatment. Presented at the Organization for Human Brain Mapping, online, 23 Jun 2020-03 Jul 2020.

4. Blommaert, J., Radwan, A., Dupont, P., Simon-Martinez, C., Van Calsteren, K., Klingels, K., Ortibus, E., Feys, H., Sunaert, S., Mailleux, L. (2020). Are subject-specific connectomics the key for understanding clinical outcomes in children with unilateral cerebral palsy? Presented at the ECMRN 2020, Marseille, 26 Feb 2020-29 Feb 2020.

5. Blommaert, J., Radwan, A., Sleurs, C., Peeters, R., Sunaert, S., Gorissen, A-S., Van Calsteren, K., Deprez, S., Amant, F. (2020). The effect of prenatal chemotherapy exposure on functional brain development: a prospective case-control functional MRI study. Presented at the ECMRN 2020, Marseille, 26 Feb 2020-29 Feb 2020.

6. Sleurs, C., Lemiere, J., Blommaert, J., Sunaert, S., Deprez, S., Uyttebroeck, A., Jacobs, S. (2020). Cortical volume in posterior fossa tumor survivors. Presented the ECMRN, Marseille, 26 Feb 2020-29 Feb 2020.

7. Mc Donald, B.C., Deardoff, R.L., West, J.D., Blommaert, J., Chen, B.T., Dumas, J., Schagen, S.B., Sunaert, S., Cimprich, B., Askren, M.K., Peltier, S., Silverman, D.H., Deprez, S., Saykin, A.J., De Ruiter, M.B. (2020). Gray matter reduction after breast cancer chemotherapy: a multicenter datapooling analysis. Presented at the ICCTF, Denver, USA, 03 Feb 2020-05 Feb 2020. 
8. Blommaert, J., van Gerwen, M., Gorissen, A-S., Deprez, S., Van Calsteren, K., Lemiere, J., van Dijk-Lokkart, E.M., Amant, F., for the International Network on Cancer, Infertili, (2020). The effects of prenatal exposure to maternal malignancy and its treatment on neurocognitive development. Presented at the ICCTF, Denver, USA, 03 Feb 2020-05 Feb 2020. Open Access

9. Blommaert, J., Radwan, A., Sleurs, C., Peeters, R., Sunaert, S., Gorissen, A-S., Van Calsteren, K., Amant, F., Deprez, S. (2020). White matter development after prenatal chemotherapy exposure: a diffusion MRI fixel-based analysis. Presented at the ISMRM Benelux Chapter meeting, Arnhem, Netherlands, 24 Jan 2020-24 Jan 2020.

10. Melis, M., Van der Gucht, K., Ahmadoun, S., de Cloe, E., Smeets, A., Vandenbulcke, M., Wildiers, H., Neven, P., Takano, K., Sleurs, C., Blommaert, J., Radwan, A., Kuppens, P., Raes, F., Sunaert, S., Deprez, S. (2019). Effects of a mindfulness-based intervention on cancer-related cognitive impairment: Results from a fMRI pilot study. Presented at the $\mathrm{CHI}$-congres in de psychosociale oncologie: Littekens bij en na kanker, Mechelen, Belgium.

11. Sleurs, C., Blommaert, J., Batalle, D., Verly, M., Sunaert, S., Peeters, R., Lemiere, J., Uyttebroeck, A., Deprez, S. (2019). Cortical thinning and altered functional coherence in survivors of childhood sarcoma. Presented at the SIOP, Lyon, France, 23 Oct 2019-25 Oct 2019.

12. Sleurs, C., Nollet, K., Gillebert, C., Blommaert, J., Radwan, A., Verly, M., Vandenabeele, K., Van Beek, K., Deprez, S., Uyttebroeck, A., Jacobs, S., Lemiere, J. (2019). Could single white matter tracts tell us something about long-term executive and motor functioning of survivors of childhood posterior fossa tumors? Presented at the SIOP, Lyon, France.

13. Radwan, A., Blommaert, J., Kovacs, S., Peeters, R., De Vleeschouwer, S., Dupont, P., Theys, T., Sunaert, S. (2019). A BIDS compliant automated CSD fiber tracking pipeline for presurgical white matter mapping. In: MAGMA: vol. 32 (Suppl 1), (Abstract No. A1407), (S299-S299). Presented at the ESMRMB, Rotterdam, NL, 03 Oct 2019-05 Oct 2019. doi: $10.1007 /$ s10334-019-00755-1

14. Emsell, L., Blommaert, J., Van Steelandt, K., Pille, W., De Winter, F-L., Deprez, S., Sunaert, S., Bouckaert, F., Vandenbulcke, M. (2019). Regional brain white matter hyperintensities and neuropsychological function in late-life depression. Presented at the ESMRMB, Rotterdam, Netherlands, 03 Oct 2019-05 Oct 2019.

15. Blommaert, J., Zink, R., Deprez, S., Myatchin, I., Dupont, P., Vandenbroucke, T., Sleurs, C., Van Calsteren, K., Amant, F., Lagae, L. (2019). Longterm impact of prenatal 
exposure to chemotherapy on attentional neurodevelopment: an ERP study. Presented at the OHBM annual meeting, Rome, Italy, 09 Jun 2019-13 Jun 2019.

16. Blommaert, J., Radwan, A., Sleurs, C., Peeters, R., Sunaert, S., Vandenbroucke, T., Schroyen, G., Van Calsteren, K., Deprez, S., Amant, F. (2019). In utero exposure to chemotherapy affects cortical neurodevelopment. Presented at the ISMRM, Montréal, Canada, 11 May 2019-16 May 2019.

17. Schroyen, G., Blommaert, J., Vandenbulcke, M., Radwan, A., Smeets, A., Peeters, R., Sleurs, C., Neven, P., Wildiers, H., Amant, F., Sunaert, S., Deprez, S. (2019). Longitudinal brain volume changes in pre-menopausal breast cancer patients treated with chemotherapy. Presented at the ISMRM, Montreal, Canada, 11 May 2019-16 May 2019.

18. Radwan, A., Blommaert, J., Mailleux, L., Simon Martinez, C., Klingels, K., Ortibus, E., Feys, H., Sunaert, S. (2018). A tailored functional MRI derived atlas: a potential tool for mapping brains with large lesions. Presented at the 2018 OHBM ANNUAL MEETING, Singapore, 17 Jun 2018-21 Jun 2018.

19. Mailleux, L., Simon Martinez, C., Radwan, A., Blommaert, J., Gooijers, J., Wenderoth, N., Klingels, K., Ortibus, E., Sunaert, S., Feys, H. (2018). White matter microstructure of motor and sensory tracts and the corpus callosum underlying impaired upper limb function in children with unilateral cerebral palsy. In: Developmental MEdicine and Child Neurology: vol. 60 (2), (Abstract No. P-52), (46-46). Presented at the European Academy of Child Disability, Tbilisi, 28 May 2018-30 May 2018.

20. Blommaert, J., Amant, F., Peeters, R., Radwan, A.M M M., Smeets, A., Sleurs, C., Sunaert, S., Vandenbulcke, M., Deprez, S. (2017). Longitudinal assessment of morphometric brain changes after chemotherapy in pre- and post-menopausal breast cancer patients. In: Magnetic Resonance Materials in Biology, Physics and Medicine: vol. 30, (432-433). Presented at the ESMRMB, Barcelona, 18 Oct 2017-21 Oct 2017.

21. Sleurs, C., Lemiere, J., Christiaens, D., Billiet, T., Verly, M., Blommaert, J., Peeters, R., Sunaert, S., Uyttebroeck, A., Deprez, S. (2017). White matter density in solid tumor survivors using advanced diffusion models. Presented at the OHBM, Vancouver, 25 Jun 2017-29 Jun 2017. 


\section{DANKWOORD}

Het lijkt een cliché, maar een doctoraat maak je niet alleen. Je kan het vergelijken met een solist die een uitdagend concert wilt opvoeren. Op een dag zoals vandaag, waar je na vier jaar werken mag tonen wat je bereikt hebt, staan alle schijnwerpers op deze solist, maar het is door de steun en inzet van vele anderen dat dit resultaat er gekomen is.

Allereerst wil ik mijn promotoren, Frédéric, Kristel en Sabine, bedanken. Zij zijn de dirigenten van het orkest, met hun kennis en aanwijzingen hebben ze deze vier jaar in goede banen geleid. Ik solliciteerde vier en een half jaar geleden op dit doctoraat omdat ik interdisciplinair onderzoek wou doen met een directe klinische impact. Ik durf dan ook met trots te zeggen dat ik dit in mijn onderzoek zeker en vast gevonden heb.

Frédéric, ik vind het oprecht prachtig hoe je op 15 jaar tijd de wens van 1 patient, die haar zwangerschap wou kunnen behouden, hebt beantwoord met een internationale samenwerking waar meer dan 2000 vrouwen opgevolgd worden, waarvan het overgrote deel behandeld wordt. Ik ben dan ook blij dat we de komende jaren samen een nieuwe uitdaging kunnen aangaan, deze keer in de vorm van protontherapie tijdens de zwangerschap.

Kristel, jij stond mee aan de wieg van het onderzoek naar kanker tijdens de zwangerschap. Zo startte jij, samen met Frédéric, de opvolging van de kinderen op, waar mijn thesis uit voortkwam. Als gynaecoloog, zet je vandaag de dag deze kennis dan ook regelmatig om in de praktijk. Ook tijdens mijn doctoraat zorgde je telkens, door de juiste kritische vragen te stellen, dat mijn onderzoek klinisch relevant bleef.

Sabine, toen ik vier jaar geleden aan dit doctoraat begon, stond je meteen klaar met een 
heel inloopplan. Je nam jouw tijd om me helemaal in te werken, zelfs wanneer ik tot mijn eigen schaamte moest toegeven dat ik het verschil tussen witte en grijze stof niet meer kende. Doorheen onze wekelijkse meetings leerde je me de nodige technische kennis, maar ook soft skills, die ik nodig had om dit doctoraat tot een goed einde te brengen.

Second in this $\mathrm{PhD}$ concert, I would like to thank the jury members, the critics of this performance. While at first, we often do not like to be confronted with the flaws in our work, this crucial feedback always improves the final result. Therefore, I would like to thank prof. Dupont, prof. Jansen, dr. Mennes and dr. Fumagalli for sharing their insights on this work. Graag dank ik ook prof. Marc Fransen en prof. Baki Topal, om de schriftelijke en mondelijke evaluatie in goede banen te leiden.

De basis van elk orkest wordt gevormd door de strijkers. Doorheen mijn doctoraat vond ik deze in de vorm van mijn collega's waar ik elke dag weer op kon rekenen of het nu was om een onverwachte uitkomst uit een analyse te verklaren, de frustratie te verwerken van een afgewezen artikel of gewoon even samen een koffie te halen. Het was dan ook geweldig dat in dit doctoraat ik deel mocht uitmaken van niet één maar wel twee verschillende teams.

Het onderzoeksteam naar kanker en zwangerschap is een internationaal en multidisciplinair team. Ondanks dat ik in de praktijk meestal mijn dagen doorbracht bij het neuroradiologie-team was het steeds fijn thuiskomen in dit team. Allereerst is er het 'leger' aan psychologen: Tineke, Cettina, Kaat, Lara, An-Sofie, Agnetha, Mathilde en Evangeline. Jullie zetten je allemaal 200\% in om de opvolging van de kinderen gaande te houden, doorheen testafnames, tests scoren, controles recruteren, de kinderdatabase aan te vullen en veel meer. Enorm bedankt ook Diane, om telkens weer die puzzel te maken om kindjes ingepland te krijgen voor al die verschillende onderzoeken, ondanks overvolle planningen van zowel de onderzoekers als de ouders en kinderen zelf. Bovenop doe je dit altijd met een grote glimlach, zelfs wanneer je na tien keer bellen nog steeds de ouders niet aan de lijn krijgt. Bedankt ook aan mijn voorganger Dorothée, je legde een mooie basis voor dit project, waardoor ik meteen aan de slag kon met het testen, met zelfs al een behoorlijk aantal kindjes in de dataset. Ik dank ook graag de artsen-onderzoekers, Magali, Jorine, Charlotte en Vera, voor al hun geduld om mij bij te leren over de dagelijkse klinische realiteit binnen de gynaecologie en oncologie. Daarenboven zal ik nooit de aanstekelijke bulderlach van Magali vergeten. Joosje, jou mocht ik door de coronacrisis de laatste maanden vooral via Zoom en Skype leren kennen. Gelukkig was er in de REDCap meetings ook al wat tijd om elkaar persoonlijk te leren kennen, inclusief beide onze verbouwingsperikelen. Liesbeth, Hanne en Ilana, jullie openden voor mij een heel nieuwe wereld aan labo-terminologie. Ook al heb ik eerlijk gezegd soms moeite met te volgen doorheen het bos van technieken die jullie beheersen, ik besef maar al te goed hoe waardevol jullie onderzoek is voor deze patiënten. Tenslotte, kunnen we altijd vertrouwen op de 
ondersteuning van Marie, Liesbeth en Katrien. Jullie houden dit team echt bij elkaar! Dankjewel ook Tine, Lynn en Laurence voor de gezellige middagpauzes wanneer ik op het labo was.

Naast het CIP-team mocht ik ook deel uitmaken van het neuroradiologie team van prof. Stefan Sunaert. Zo kon ik van het MIRC mijn vaste uitvalsbasis maken. Stefan, enorm bedankt om mij in jouw team mee op te nemen en mij de kneepjes van de MRI beeldvorming en verwerking te leren. Charlotte, jij was vanaf dag één mijn partner in crime. Je bent een echte allround neurowetenschapper, en ik heb dan ook enorm veel van jou geleerd: van scannen op MR8, tot leren werken met terminal tot eindeloze inhoudelijke discussies over hoe we een analyse best aanpakken. Marjolein, ook jij was er van in het begin. Ik leerde je snel kennen als een ambitieuze en no-nonsense vrouw. De COSMO vaardigheden, die jij mij leerde, hebben niet alleen mijn beeldkwaliteit zeker en vast verbeterd, maar maakten van de MRI toch een veel aangenamere ervaring voor al die kinderen. Ilse, enorm bedankt voor me al die keren dat ik aan de scanner stond en weer vergeten was mijn bon te laten receptioneren. Naast jouw duidelijke mening, waardeer ik vooral je Limburgse gezelligheid. Sil, ook jij hebt een enorm rechtuite mening en open minded attitude. Je brengt dan ook enorm veel leven aan het eiland. Dank je dat ik op jou kon rekenen wanneer mijn MR-planning helemaal in de knoop lag, en je de sporen van dubbelgeboekte sloten moest wissen.

Mettertijd werd dit team alsmaar groter. Als eerste aanwinst mochten we Ahmed verwelkomen. Not only at work we make a great team, but through gardening, bacon and beers we became true bro's. At my wedding you even made an everlasting impression on my family in law. lets later kwam ook Gwen erbij. Meteen hielp je mee de laatste loodjes aan het DBM-artikel te leggen. Enorm bedankt om me mee door al die revisies te loodsen. Gelukkig, is dan uiteindelijk het artikel gepubliceerd geraakt en zijn we er bovendien als goede maatjes uitgekomen. Soumaya, ook al was je maar een jaartje bij ons, je hebt zeker een blijvende indruk gemaakt. Sorry ook voor al die slechte dad-jokes, maar ik zal ze toch blijven maken. Na drie jaar het jonkie van de groep te zijn, kwam Michelle er bij, een enorm empathische en sociale vrouw. Sinds jouw komst is het aantal afterwork ijsjes of borrels dan ook gestaag toegenomen. Als nieuwste lid kwam Nicolas ons vervoegen. Met je scherpe humor en aanstekelijke persoonlijkheid werd ook jij al snel een vaste waarde. Er zijn dan ook nog twee 'outsiders' in het MIRC die ik ook graag even bedank. Louise, it is always nice when you pop around the MIRC. The recent psychiatry seat at the island will hopefully lead to a lot of new glue meetings and projects. Joke, ook jij bent een beetje outsider in de groep, al kunnen we je ondertussen toch ook wel al bij het eiland rekenen. Je bent enorm gepassioneerd door jouw werk, het hoefde dan ook niet te verbazen dat na enkele koffiepauzes je Ahmed en mij mee inzette voor de PESA studie. Bedankt alle collega's in het MIRC, te veel om op te sommen, voor de deugdoende pauzes in het koffiekot. Dankjewel ook aan Ron en de studiemannen, Guido, Kris en Stefan, alsook Tom en Frederik, voor al die keren 
dat ik jullie naar MR8 liet lopen omdat de scanner 'kapot' was. Destemeer ook mijn oprechte excuses, voor al die keren dat deze problemen nogal banaal waren.

Vervolgens is er de blazerssectie van het orkest. Zij geven af en toe wat nieuwe kleur aan het stuk. Ook in dit doctoraat waren er enkele fijne samenwerkingen. Vooral hoofdstuk 5 was hierdoor gekenmerkt. Zo leerden Ivan en de dames van het EEG-lab, An, Birgit, en Marleen de kneepjes van het vak om mijn ERP-experimenten tot een goed einde te brengen. Rob, met jouw hulp konden we uit deze, vaak ruizige, data vervolgens waardevolle ERP-metingen krijgen. Prof. Lieven Lagae, u lag mee aan de basis van dit ERP-avontuur, bedankt om het ook mee tot een goed einde te helpen. Ook prof. Dupont had ik graag nogmaals bedankt. Als jurylid bleef u niet altijd aan de zijlijn staan, maar uw input in de statistiek van het ERP-project en de graph theory analyse hebben een enorme meerwaarde aan beide hoofdstukken gegeven. Daan, bedankt om de preprocessing van de diffusiebeelden mee op punt te zetten, om zo toch het maximale uit de data te kunnen halen. Ten slotte was er ook de samenwerking met de cerebral palsy onderzoeksgroep. Lisa, Cristina, Hilde, Els en Katrijn enorm bedankt voor deze samenwerking waaruit alvast 2 mooie publicaties en enkele toekomstige projecten zijn voortgekomen.

Elk orkest heeft een goede ondersteunende bassectie nodig. Zij zoeken zelden de voorgrond op in deze compositie, maar vormen de basis waarop dit werk kan voortbouwen. Voor wie mij goed kent, weet dan ook dat deze instrumentengroep een speciaal plekje in mijn hart heeft. In dit doctoraat vormen mijn vrienden en familie deze sectie. De ingenieurs-spirit kreeg ik van kindsaf mee van mijn ouders en broers, dus was het misschien niet verwonderlijk dat ook ik uiteindelijk een 'denk'-ingenieur ben geworden. Al zorgde de keuze om dit toe te passen in het medische toch wel voor een aangename verfrissing. Ook kreeg ik er een paar jaar geleden een geweldige schoonfamilie bij. Bij hen zijn alle mogelijke cliché's over kwaadaardige schoonmoeders en dergelijke zeker niet van toepassing, want zij zijn ook steevast mijn grootste supporters.

Vooraan in deze bassectie staat er uiteraard mijn vrouw, Isabeau. Hoewel een doctoraat tot een goed einde brengen al een hele uitdaging op zich is, was dit toch niet voldoende voor ons. In de afgelopen vier jaar besloten we dan ook een huis te kopen, dit vervolgens helemaal op te knappen (tuin inclusief) en in het huwelijksbootje te stappen. Na ons eerder al door drie masterthesissen te slaan ondersteunde je me ook enorm in dit vierjarig avontuur. Ik ben dan ook zeker dat we elke uitdaging die we in de toekomst aangaan met succes zullen doorstaan.

Elke opvoering is maar geslaagd wanneer het zijn publiek kan ontroeren. In het geval van dit doctoraat bestaat dit publiek uiteraard uit de clinici en patiënten die elke dag geconfronteerd worden met de moeilijke beslissingen, die een kankerdiagnose tijdens de zwangerschap met zich meebrengt. Ik hoop dan ook dat dit onderzoek een verschil voor hen kan maken. 
Tenslotte, behoren de ereplaatsen in dit publiek toe aan alle families die hebben deelgenomen aan mijn studie. Zonder de toewijding van deze kinderen, die hun haar vol lieten smeren met gel voor een ERP-experiment of een half uur moesten stil liggen in de MRI/donut, had dit onderzoek er nooit geweest.

Dankjewel allemaal voor een fantastische vier jaar! 